\title{
Numerical Investigation of LAminar-Turbulent Transition in a Cone Boundary Layer at Mach 6 \\ by \\ Jayahar Sivasubramanian
}

A Dissertation Submitted to the Faculty of the

Department of Aerospace and Mechanical Engineering

In Partial Fulfillment of the Requirements

For the Degree of

Doctor of PHILOSOPHY

With a Major in Aerospace Engineering

In the Graduate College

The University of Arizona

2012 


\section{THE UNIVERSITY OF ARIZONA GRADUATE COLLEGE}

As members of the Dissertation Committee, we certify that we have read the dissertation prepared by Jayahar Sivasubramanian

entitled "Numerical Investigation of Laminar-Turbulent Transition in a Cone Boundary Layer at Mach 6"

and recommend that it be accepted as fulfilling the dissertation requirement for the Degree of Doctor of Philosophy

Date: $04 / 23 / 12$

Hermann F. Fasel

Date: $04 / 23 / 12$

Moysey Brio

Date: $04 / 23 / 12$

Jeffrey W. Jacobs

Date: $04 / 23 / 12$

Edward J. Kerschen

Final approval and acceptance of this dissertation is contingent upon the candidate's submission of the final copies of the dissertation to the Graduate College.

I hereby certify that I have read this dissertation prepared under my direction and recommend that it be accepted as fulfilling the dissertation requirement.

Dissertation Director: Hermann F. Fasel

Date: $04 / 23 / 12$ 


\section{STATEMENT BY AUTHOR}

This dissertation has been submitted in partial fulfillment of requirements for an advanced degree at The University of Arizona and is deposited in the University Library to be made available to borrowers under rules of the Library.

Brief quotations from this dissertation are allowable without special permission, provided that accurate acknowledgment of source is made. Requests for permission for extended quotation from or reproduction of this manuscript in whole or in part may be granted by the head of the major department or the Dean of the Graduate College when in his or her judgment the proposed use of the material is in the interests of scholarship. In all other instances, however, permission must be obtained from the author. 


\section{ACKNOWLEDGMENTS}

First and foremost, I would like to thank my advisor, Professor Hermann F. Fasel for giving me the opportunity to study and do research in the field of computational fluid dynamics at the University of Arizona. I am especially grateful for his patience, support and guidance during these past seven years. Special thanks also go to my dissertation committee members, Professors Moysey Brio, Jeffrey W. Jacobs and Edward J. Kerschen, for their valuable advice and expert input on my dissertation research. I would also like to thank Professor Anatoli Tumin for serving on the committee for my oral comprehensive exam.

I am very thankful to Dr. Andreas Laible for providing the compressible NavierStokes solver which was used to perform the stability and transition simulations presented in this dissertation. I also want to thank Dr. Christian Mayer for all the good times in the office and all the discussions about compressible linear stability theory, wave packets and other important things about life. Moreover, I thank all my colleagues and friends who have been part of the CFD group during the past seven years for making my time in Tucson enjoyable. I also want to acknowledge Barbara Heefner, Aurora Rau, Dianne Smith, and Judee Aten at AME for their help.

This dissertation research was funded by the Air Force Office of Scientific Research under grant FA9550-08-1-0211 with Dr. John Schmisseur serving as program manager, and the AFOSR/NASA National Center for Hypersonic Laminar-Turbulent Transition Research at the Texas A\&M University. Computer time provided by the DoD High Performance Computing Modernization Program is also gratefully acknowledged.

Last but not least, I want to thank my parents and my sister Jayapriya for their moral and financial support during my graduate studies in the United States. 


\section{DEDICATION}

To my wonderful parents

K. Sivasubramanian and S. Prabha

For all their love, support and encouragement. 


\section{TABLE OF CONTENTS}

LIST OF FIGURES . . . . . . . . . . . . . . . . . . . . . . . . . . 9

LIST OF TABLES . . . . . . . . . . . . . . . . . . . . . . 23

NOMENCLATURE . . . . . . . . . . . . . . . . . . 24

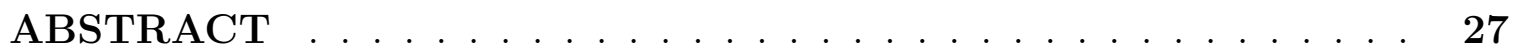

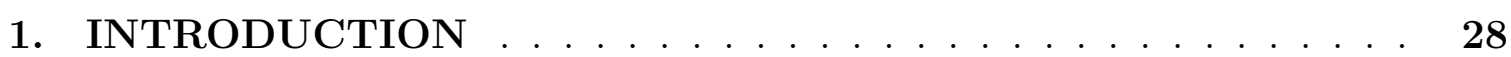

1.1 Motivation . . . . . . . . . . . . . . . . . . 28

1.2 Laminar-Turbulent Transition in Boundary Layers . . . . . . . . . . 32

1.3 Flow Past a Circular Sharp Cone at Zero Angle of Attack . . . . . . . 35

1.4 Scope of Present Research . . . . . . . . . . . . . . . . . . . 37

2. GOVERNING EQUATIONS AND NUMERICAL METHODS . 43

2.1 Governing Equations . . . . . . . . . . . . . . . . . . . 43

2.2 Time Integration . . . . . . . . . . . . . . . . . . . . 46

2.3 Spatial Discretization . . . . . . . . . . . . . . . . 47

2.4 Boundary Conditions . . . . . . . . . . . . . . . . . . . . 48

2.4.1 Inflow Boundary Condition . . . . . . . . . . . . . 48

2.4.2 Wall Boundary Condition . . . . . . . . . . . 48

2.4.3 Outflow Boundary Condition . . . . . . . . . . . . 49

2.4.4 Free-stream Boundary Condition . . . . . . . . . . . . . . 49

2.5 Disturbance Generation . . . . . . . . . . . . . . . . . . . . . . 49

2.5.1 pulse forcing . . . . . . . . . . . . . . . 49

2.5 .2 harmonic forcing . . . . . . . . . . . . . . 50

3. AXISYMMETRIC CONE BASE FLOW . . . . . . . . . . . . 52

3.1 Flow Parameters and Physical Problem . . . . . . . . . . . . . . 52 


\section{TABLE OF CONTENTS-Continued}

3.2 Simulation Strategy . . . . . . . . . . . . . . . . . . . . 53

4. THREE DIMENSIONAL WAVE PACKET SIMULATIONS . . 56

4.1 Simulation Setup . . . . . . . . . . . . . . . 56

4.2 Linear Wave Packet . . . . . . . . . . . . . . . . . . . . . 58

4.2.1 Development of the Wave Packet . . . . . . . . . . . . . . 59

4.2 .2 Disturbance Field . . . . . . . . . . . . . . . . . . . 65

4.2.3 Comparison with flat plate . . . . . . . . . . . . 80

4.3 Weakly Nonlinear Wave Packet . . . . . . . . . . . . . . . . . . 92

4.3.1 Development of the wave packet . . . . . . . . 93

4.3 .2 Disturbance Field . . . . . . . . . . . . . . . . . . . . . . . 96

4.3.3 Comparison with flat plate . . . . . . . . . . . . . . . . 101

4.4 Strongly Nonlinear Wave Packet . . . . . . . . . . . . . . . . 107

4.4.1 Initial development of the wave packet . . . . . . . . 107

4.4.2 Flow structures within the turbulent spot . . . . . . . . . 113

4.4.3 General features of the turbulent spot . . . . . . . . . . 128

4.5 Summary of Wave Packet Simulations . . . . . . . . . . . . . . . . . 131

5. CONTROLLED TRANSITION SIMULATIONS . . . . . . . . . 134

5.1 Parameter Study . . . . . . . . . . . . . . . . . . . 135

5.1.1 Fundamental Resonance . . . . . . . . . . . . . . . 135

5.1 .2 Subharmonic Resonance . . . . . . . . . . . . . . . . 139

5.2 Fundamental Breakdown . . . . . . . . . . . . . . . . . . . . 142

5.3 Oblique Breakdown . . . . . . . . . . . . . . . . . . . 158

5.4 Summary of controlled transition simulations . . . . . . . . . . . . . 169

6. SUMMARY AND CONCLUSIONS . . . . . . . . . . . . 174 
TABLE OF CONTENTS-Continued REFERENCES . . . . . . . . . . . . . . . . 178 


\section{LIST OF FIGURES}

$1.1 \quad \mathrm{X}-43 \mathrm{~A}$ hypersonic research vehicle. . . . . . . . . . . . . . . . 29

1.2 Laminar-Turbulent transition paths in boundary layers. . . . . . . . . . 33

1.3 Shadowgraph of transition on a sharp cone at Mach 4.31. . . . . . . . 36

2.1 Wall-normal velocity at the wall (a) as a function of streamwise and azimuthal direction (left) and (b) as a function of time (right). In (a), the dashed line (- -) represents the azimuthal distribution and the solid line (-) represents the streamwise distribution of the wall-normal velocity. .

2.2 Schematic of the streamwise wall normal velocity disturbance distribution in the forcing slot used for introducing controlled disturbances. . . . . .

3.1 Comparison of streamwise velocity profiles computed by the precursor finite-volume code, the high-order code and the Mangler transformed flat plate similarity profile. . . . . . . . . . . . .

3.2 Comparison of temperature profiles computed by the precursor finitevolume code, the high-order code and the Mangler transformed flat plate similarity profile. . . . . . . . . . . . . . . . .

3.3 Comparison of the boundary layer thickness between the cone base flow simulations and a Mangler transformed flat plate similarity profile. . . .

4.1 Cone model (a) with the computational domain and (b) cross section. . 57

4.2 Temporal and spatial evolution of wall-pressure disturbance amplitude along the centerline for the linear wave packet. Solid lines represent the time signal and dashed lines its envelope. Isothermal Wall . . . . . . . .

4.3 Temporal and spatial evolution of wall-pressure disturbance amplitude along the centerline for the linear wave packet. Solid lines represent the time signal and dashed lines its envelope. Adiabatic Wall . . . . . . . . 


\section{LIST OF FIGURES-Continued}

4.4 Snapshots of the linear wave packet showing the initial flow response to forcing through a hole on the cone surface. Shown are contours of wallpressure disturbance on the unrolled cone surface at (a) $t^{*}=0.005 \mathrm{~ms}$ (b) $t^{*}=0.016 \mathrm{~ms}(\mathrm{c}) t^{*}=0.028 \mathrm{~ms}$ and $(\mathrm{d}) t^{*}=0.039 \mathrm{~ms}$. All the plots have the same contour levels. Isothermal Wall. . . . . . . . . . . . . . .

4.5 Snapshots of the linear wave packet showing the initial flow response to forcing through a hole on the cone surface. Shown are contours of wallpressure disturbance on the unrolled cone surface at (a) $t^{*}=0.005 \mathrm{~ms}$ (b) $t^{*}=0.016 \mathrm{~ms}(\mathrm{c}) t^{*}=0.028 \mathrm{~ms}$ and $(\mathrm{d}) t^{*}=0.039 \mathrm{~ms}$. All the plots have the same contour levels. Adiabatic Wall. . . . . . . . . . . . . . .

4.6 Snapshots of the linear wave packet illustrating its development in downstream direction. Shown are contours of wall-pressure disturbance on the unrolled cone surface at (a) $t^{*}=0.056 \mathrm{~ms}$ (b) $t^{*}=0.171 \mathrm{~ms}$ and (c) $t^{*}=0.285 \mathrm{~ms}$. All the plots have the same contour levels. Isothermal Wall. 66

4.7 Snapshots of the linear wave packet illustrating its development in downstream direction. Shown are contours of wall-pressure disturbance on the unrolled cone surface at (a) $t^{*}=0.056 \mathrm{~ms}$ (b) $t^{*}=0.171 \mathrm{~ms}$ and (c) $t^{*}=0.285 \mathrm{~ms}$. All the plots have the same contour levels. Adiabatic Wall.

4.8 Close-up view of the linear wave packet at time $t^{*}=0.4 \mathrm{~ms}$. Shown are contours of wall-pressure disturbance using different contour levels for visualization. Isothermal Wall. . . . . . . . . . . . . . . .

4.9 Close-up view of the linear wave packet at time $t^{*}=0.4 \mathrm{~ms}$. Shown are contours of wall-pressure disturbance using different contour levels for visualization. Adiabatic Wall. . . . . . . . . . . . . . .

4.10 Contours of constant amplification rate $\alpha_{i}$ for axisymmetric disturbances $\left(k_{c}=0\right)$. Generated using Mack's LST solver. Isothermal Wall. . . . . . 


\section{LIST OF FIGURES-Continued}

4.11 Contours of constant amplification rate $\alpha_{i}$ for axisymmetric disturbances $\left(k_{c}=0\right)$. Generated using Mack's LST solver. Adiabatic Wall. . . . . . .

4.12 Comparison of spatial growth rates of axisymmetric disturbance waves $\left(k_{c}=0\right)$ obtained for adiabatic and isothermal wall cases. Growth rates are obtained using Mack's LST solver and are plotted as a function of reduced frequency $F \ldots \ldots \ldots \ldots \ldots$

4.13 Downstream development of the complex streamwise wavenumber predicted by DNS and LST. Streamwise wave number $\left(\alpha_{r}\right)$ and spatial growth rates $\left(\alpha_{i}\right)$ are compared for four axisymmetric disturbance waves $\left(k_{c}=0\right)$ with different frequencies. LST results are computed using Mack's solver. Isothermal Wall. . . . . . . . . . . . . . . . . . . . . . . . .

4.14 Downstream development of the complex streamwise wavenumber predicted by DNS and LST. Streamwise wave number $\left(\alpha_{r}\right)$ and spatial growth rates $\left(\alpha_{i}\right)$ are compared for four axisymmetric disturbance waves $\left(k_{c}=0\right)$ with different frequencies. LST results are computed using Mack's solver. Adiabatic Wall. . . . . . . . . . . . . . . . . . . . . . .

4.15 Comparison of wall-normal amplitude and phase distribution of the streamwise velocity and pressure disturbance to theoretical predictions from LST for frequency $F=1.04 E-04$ and azimuthal mode number $k_{c}=0$ (axisymmetric disturbance wave) at $R_{x}=2600$. LST results are computed using Mack's solver. Adiabatic wall.

4.16 Disturbance spectrum in the azimuthal mode number - frequency $\left(k_{c}-f^{*}\right)$ plane at the forcing location $\left(x^{*}=0.138 m\right)$ for the simulation with low forcing amplitude. Obtained from isothermal (left) and adiabatic (right) wall cases. . . . . . . . . . . . . . . . . . . . . . . 


\section{LIST OF FIGURES-Continued}

4.17 Disturbance spectrum in the azimuthal mode number - frequency $\left(k_{c}-f^{*}\right)$ plane for the simulation with low forcing amplitude obtained from wallpressure at different streamwise positions. (a) $x^{*}=0.250 \mathrm{~m}$, (b) $x^{*}=$ $0.350 \mathrm{~m},(\mathrm{c}) x^{*}=0.450 \mathrm{~m}$, and (d) $x^{*}=0.550 \mathrm{~m}$. All the plots have the same contour levels. Isothermal wall. . . . . . . . . . . . . . .

4.18 Disturbance spectrum in the azimuthal mode number - frequency $\left(k_{c}-f^{*}\right)$ plane for the simulation with low forcing amplitude obtained from wallpressure at different streamwise positions. (a) $x^{*}=0.250 \mathrm{~m}$, (b) $x^{*}=$ $0.350 \mathrm{~m},(\mathrm{c}) x^{*}=0.450 \mathrm{~m}$, and $(\mathrm{d}) x^{*}=0.550 \mathrm{~m}$. All the plots have the same contour levels. Adiabatic wall. . . . . . . . . . . . . .

4.19 Temporal and spatial evolution of wall-pressure disturbance amplitude along the centerline for the linear wave packet on a flat-plate. Solid lines (-) represent the time signal and dashed lines (- - -) its envelope. Isothermal wall.

4.20 Contours of constant amplification rate $\alpha_{i}$ for 2D disturbances $(k=0)$. Obtained using Mack's LST solver. Isothermal wall. . . . . . . . . .

4.21 Comparison of streamwise wavelength $\left(\alpha_{r}\right)$ and spatial growth rates $\left(\alpha_{i}\right)$ for three 2D disturbance waves with different frequencies. Lines are from DNS and symbols are from LST. LST results are computed using Mack's solver. Isothermal Wall. . . . . . . . . . . . . . . . . . . . .

4.22 Comparison of wall-normal amplitude and phase distribution of the streamwise velocity, temperature and pressure disturbance to theoretical predictions from LST for frequency $F=6.2246 E-05$ and spanwise mode number $k=0$ at $R_{x}=2500$. LST results are computed using Mack's solver. Isothermal Wall. 


\section{LIST OF FIGURES-Continued}

4.23 Snapshots of the linear wave packet showing the initial flow response to pulse forcing. Shown are contours of wall-pressure disturbance at (a) $t^{*}=0.006 m s(\mathrm{~b}) t^{*}=0.017 \mathrm{~ms}$ (c) $t^{*}=0.029 \mathrm{~ms}$ and (d) $t^{*}=0.041 \mathrm{~ms}$. All the plots have the same contour levels. Isothermal Wall. . . . . . .

4.24 Snapshots of the linear wave packet illustrating its development in downstream direction. Shown are contours of wall-pressure disturbance at (a) $t^{*}=0.064 \mathrm{~ms}(\mathrm{~b}) t^{*}=0.174 \mathrm{~ms}$ and $(\mathrm{c}) t^{*}=0.291 \mathrm{~ms}$. All the plots have the same contour levels. Isothermal Wall. . . . . . . . . . . . . .

4.25 Comparison of linear wave packets in a flat-plate and a sharp cone boundary layer. Shown are contours of wall-pressure disturbance for (a) a flatplate and (b) a sharp cone at $t^{*}=0.4 m s$. Isothermal Wall. . . . . . .

4.26 Disturbance spectrum in the spanwise mode number - frequency $\left(k-f^{*}\right)$ plane obtained at the forcing location $\left(x^{*}=0.138 m\right)$ for the simulation with low forcing amplitude. Isothermal wall. . . . . . . . . . . . .

4.27 Disturbance spectrum in the spanwise mode number - frequency $\left(k-f^{*}\right)$ plane for the simulation with low forcing amplitude obtained from wallpressure at different streamwise positions. (a) $x^{*}=0.250 m$, (b) $x^{*}=$ $0.300 m,(\mathrm{c}) x^{*}=0.350 m,(\mathrm{~d}) x^{*}=0.400 m$, (e) $x^{*}=0.450 m$ and (f) $x^{*}=0.500 m$. Isothermal Wall. . . . . . . . . . . . . . .

4.28 Temporal and spatial evolution of wall-pressure disturbance amplitude along the centerline for the weakly nonlinear wave packet. Isothermal Wall. 94

4.29 Temporal and spatial evolution of wall-pressure disturbance amplitude along the centerline for the weakly nonlinear wave packet. Adiabatic wall. 95 


\section{LIST OF FIGURES-Continued}

4.30 Snapshots of the weakly nonlinear wave packet illustrating its development in downstream direction. Shown are contours of wall-pressure disturbance on the unrolled cone surface at (a) $t^{*}=0.40 \mathrm{~ms}$, (b) $t^{*}=0.46 \mathrm{~ms}$ and $(\mathrm{c}) t^{*}=0.51 \mathrm{~ms}$. All the plots have the same contour levels. Isothermal Wall. . . . . . . . . . . . . . . . . . . . . . . .

4.31 Snapshots of the weakly nonlinear wave packet illustrating its development in downstream direction. Shown are contours of wall-pressure disturbance on the unrolled cone surface at (a) $t^{*}=0.40 \mathrm{~ms}$, (b) $t^{*}=0.46 \mathrm{~ms}$ and $(\mathrm{c}) t^{*}=0.51 \mathrm{~ms}$. All the plots have the same contour levels. Adiabatic

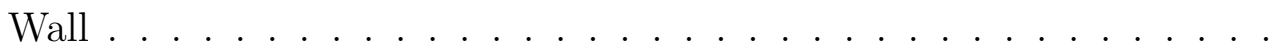

4.32 Disturbance spectrum in the azimuthal mode number - frequency $\left(k_{c}-f^{*}\right)$ plane, obtained from wall-pressure at different streamwise positions. (a) $x^{*}=0.250 m,(\mathrm{~b}) x^{*}=0.350 m$, (c) $x^{*}=0.450 m$, (d) $x^{*}=0.550 m,(\mathrm{e})$ $x^{*}=0.580 m$. All the plots have the same contour levels. Isothermal wall.

4.33 Disturbance spectrum in the azimuthal mode number - frequency $\left(k_{c}-f^{*}\right)$ plane, obtained from wall-pressure at different streamwise positions. (a) $x^{*}=0.250 m,(\mathrm{~b}) x^{*}=0.350 m$, (c) $x^{*}=0.450 m$, (d) $x^{*}=0.550 m,(\mathrm{e})$ $x^{*}=0.580 \mathrm{~m}$. All the plots have the same contour levels. Adiabatic wall.

4.34 Temporal and spatial evolution of wall-pressure disturbance amplitude along the centerline for the nonlinear wave packet. Solid lines $(-)$ represent the time signal and dashed lines (- - -) its envelope. Isothermal wall. . . . . . . . . . . . . . . . . . . 103

4.35 Snapshots of the nonlinear wave packet illustrating its development in downstream direction. Shown are contours of wall-pressure disturbance at (a) $t^{*}=0.17 \mathrm{~ms}$, (b) $t^{*}=0.29 \mathrm{~ms}$, (c) $t^{*}=0.41 \mathrm{~ms}$ and (d) $t^{*}=0.52 \mathrm{~ms}$. All the plots have the same contour levels. Isothermal Wall. . . . . . . . 105 


\section{LIST OF FIGURES-Continued}

4.36 Disturbance spectrum in the spanwise mode number - frequency $\left(k-f^{*}\right)$ plane for the simulation with higher forcing amplitude obtained from wallpressure at different streamwise positions. (a) $x^{*}=0.250 \mathrm{~m}$, (b) $x^{*}=$ $0.300 m,(\mathrm{c}) x^{*}=0.350 m,(\mathrm{~d}) x^{*}=0.400 m$, (e) $x^{*}=0.450 m$ and (f) $x^{*}=0.500 m \ldots \ldots \ldots \ldots \ldots \ldots \ldots$

4.37 Temporal and spatial evolution of wall-pressure disturbance amplitude along the centerline for the strongly nonlinear wave packet. The locations of the front and the tail of the wave packet are indicated by dashed lines. Adiabatic wall. . . . . . . . . . . . . . . . . . . 108

4.38 Comparison of spatial growth rates $\left(\alpha_{i}\right)$ for three 2D disturbance waves with frequencies (a) $F=1.22 E-04$ (一, o) (b) $F=1.32 E-04(---$, $\square)$ and (c) $F=1.42 E-04(-.-.-, \diamond)$. Lines are DNS results and symbols are LST results. LST results are computed using Mack's solver. Adiabatic Wall. . . . . . . . . . . . . . . . . . . . . . . . . 109

4.39 Snapshots of the strongly nonlinear wave packet corresponding to the centerline wall-pressure disturbance distributions shown in figure 4.37. Shown are contours of wall-pressure disturbance on the unrolled cone surface at (a) $t^{*}=0.091 m s$ (b) $t^{*}=0.183 m s$ (c) $t^{*}=0.274 m s$ and (d) $t^{*}=0.366 m s$. Adiabatic Wall. . . . . . . . . . . . . . . . 111

4.40 Disturbance spectrum in the azimuthal mode number - frequency $\left(k_{c}-\right.$ $\left.f^{*}\right)$ plane obtained from wall-pressure disturbance at various streamwise positions. (a) $x^{*}=0.20 m$, (b) $x^{*}=0.24 m$, (c) $x^{*}=0.28 m$, (d) $x^{*}=$ $0.32 \mathrm{~m}$ and $(\mathrm{e}) x^{*}=0.36 \mathrm{~m}$. Adiabatic wall. . . . . . . . . . . 112 


\section{LIST OF FIGURES-Continued}

4.41 Visualization of spot using total vorticity iso-surface. Shown are side view and top view of total vorticity iso-surface at $t^{*}=0.366 \mathrm{~ms}$ on the unrolled cone surface. Iso-surface is coloured with streamwise velocity magnitude. Both plots have the same total vorticity magnitude of 500 . Adiabatic Wall. . . . . . . . . . . . . . . . . . . . . 114

4.42 Visualization of spot using total vorticity iso-surface. Shown are side view and top view of total vorticity iso-surface at $t^{*}=0.4 \mathrm{~ms}$ on the unrolled cone surface. Iso-surface is coloured with streamwise velocity magnitude. Both plots have the same total vorticity magnitude of 500. Adiabatic wall. 115

4.43 Visualisation of a "young" turbulent spot at $t^{*}=0.423 \mathrm{~ms}$. Shown are (a) end view, (b) side view and (c) top view of total vorticity iso-surfaces on the unrolled cone surface. Iso-surfaces are coloured with streamwise velocity magnitude. All the plots have the same total vorticity magnitude of 600 . Adiabatic wall. . . . . . . . . . . . . . . . .

4.44 Visualisation of a "mature" turbulent spot at $t^{*}=0.490 \mathrm{~ms}$. Shown are (a) end view, (b) side view and (c) top view of total vorticity iso-surfaces on the unrolled cone surface. Iso-surfaces are coloured with streamwise velocity magnitude. All the plots have the same total vorticity magnitude of 700. Adiabatic Wall. . . . . . . . . . . . . . . . . . . 118

4.45 (a) Perspective view of the turbulent spot and (b) Close-up view of the turbulent core region of the spot. Shown are iso-surface of total vorticity on the unrolled cone surface at $t^{*}=0.490 \mathrm{~ms}$. Iso-surfaces are coloured with streamwise velocity magnitude. Both plots have the same total vorticity magnitude of 700. Adiabatic Wall. . . . . . . . . . . . . . . . . . 119 


\section{LIST OF FIGURES-Continued}

4.46 Contours of streamwise vorticity in $y-\varphi$ plane obtained for five streamwise locations at $t^{*}=0.423 \mathrm{~ms}$. (a) $x^{*}=0.437 \mathrm{~m}$, (b) $x^{*}=0.445 \mathrm{~m}$, (c) $x^{*}=0.453 m$, (d) $x^{*}=0.461 m$ and (e) $x^{*}=0.469 m$. All the plots have the same contour levels. Adiabatic wall. . . . . . . . . . . . . . 122

4.47 Contours of streamwise vorticity in $y-\varphi$ plane obtained for five streamwise locations at $t^{*}=0.490 \mathrm{~ms}$. (a) $x^{*}=0.469 \mathrm{~m}$, (b) $x^{*}=0.486 \mathrm{~m}$, (c) $x^{*}=0.496 m$, (d) $x^{*}=0.510 m$ and (e) $x^{*}=0.526 m$. All the plots have the same contour levels. Adiabatic wall. . . . . . . . . . . . . . 123

4.48 Contours of spanwise vorticity on the $x-y$ plane for five azimuthal positions at $t^{*}=0.423 \mathrm{~ms}$. (a) $\varphi=0$, (b) $\varphi=0.048$, (c) $\varphi=0.077$, (d) $\varphi=0.097$ and (e) $\varphi=0.117$ (from bottom to top). All the plots have the same contour levels. Adiabatic Wall. . . . . . . . . . . . . . . . . 12

4.49 Contours of spanwise vorticity on the $x-y$ plane for five azimuthal positions at $t^{*}=0.490 \mathrm{~ms}$. (a) $\varphi=0$, (b) $\varphi=0.048$, (c) $\varphi=0.077$, (d) $\varphi=0.097$ and (e) $\varphi=0.117$ (from bottom to top). All the plots have the same contour levels. Adiabatic Wall. . . . . . . . . . . . . . . . .

4.50 Contours of wall-normal density gradient on the $x-y$ plane for five azimuthal positions at $t^{*}=0.423 \mathrm{~ms}$. (a) $\varphi=0$, (b) $\varphi=0.048$, (c) $\varphi=0.077$, (d) $\varphi=0.097$ and (e) $\varphi=0.117$ (from bottom to top). All the plots have the same contour levels. Adiabatic Wall. . . . . . . . . . 126

4.51 Contours of wall-normal density gradient on the $x-y$ plane for five azimuthal positions at $t^{*}=0.490 \mathrm{~ms}$. (a) $\varphi=0$, (b) $\varphi=0.048$, (c) $\varphi=0.077$, (d) $\varphi=0.097$ and (e) $\varphi=0.117$ (from bottom to top). All the plots have the same contour levels. Adiabatic Wall. 


\section{LIST OF FIGURES-Continued}

4.52 Skin friction coefficient as function of streamwise distance at $t^{*}=0.490 \mathrm{~ms}$. Data was averaged in spanwise direction over $|\varphi|<0.148 \mathrm{rad}$. Laminar and turbulent skin-friction coefficients are shown for comparison. Adiabatic Wall. . . . . . . . . . . . . . . . . . . . . . . .

4.53 Van Driest transformed streamwise velocity profiles normalized with the friction velocity in the spot core at $t^{*}=0.490 \mathrm{~ms}$. Spanwise averaged profiles over $|\varphi|<0.148 \mathrm{rad}$ at several streamwise locations within the spot core $(0.49 \leq x \leq 0.51)$. The dotted line in red indicate the linear sublayer and the dotted line in black indicate the law of the wall. Adiabatic Wall.

5.1 N-factor curves for axisymmetric waves $\left(k_{c}=0\right)$ obtained from the lowamplitude wave packet simulation.

5.2 Maximum of streamwise velocity disturbance versus downstream distance for the cases with azimuthal wave number $k_{c}=70$ (top left), $k_{c}=90$ (top right), $k_{c}=110$ (center left), $k_{c}=130$ (center right), $k_{c}=150$ (bottom left) and $k_{c}=170$ (bottom right). Shown are selected modes, which play an important role in the early nonlinear stage of fundamental resonance.

5.3 Fundamental secondary growth rate $(\sigma)$ as a function of azimuthal mode number $\left(k_{c}\right) \ldots \ldots \ldots \ldots \ldots \ldots \ldots$

5.4 Maximum of streamwise velocity disturbance versus downstream distance for the cases with azimuthal wave number $k_{c}=60$ (left) and $k_{c}=80$ (right). Shown are selected modes, which play an important role in the early nonlinear stage of subharmonic resonance. . . . . . . . . . . . . . . 140

5.5 Subharmonic secondary growth rate $(\sigma)$ as a function of azimuthal mode number $\left(k_{c}\right) \ldots \ldots \ldots \ldots \ldots \ldots \ldots$

5.6 Fundamental and subharmonic secondary growth rates $(\sigma)$ as a function of azimuthal mode number $\left(k_{c}\right)$. . . . . . . . . . . . . . . . 141 


\section{LIST OF FIGURES-Continued}

5.7 Disturbance spectrum in the azimuthal mode number - frequency $\left(k_{c}-f^{*}\right)$ plane from the weakly nonlinear wave packet simulation. Obtained from wall-pressure disturbance at $x^{*}=0.58 m \ldots \ldots$. . . . . . . . . 142

5.8 Streamwise development of the maximum u-velocity disturbance amplitude from CFUND 1. . . . . . . . . . . . . . . . . . . . 146

5.9 Time and azimuthal averaged skin friction coefficient from CFUND 1. . 147

5.10 Streamwise development of the maximum u-velocity disturbance amplitude from CFUND 2. . . . . . . . . . . . . . . . . . . . . . 149

5.11 Streamwise development of the maximum u-velocity disturbance amplitude from CFUND $3 . \ldots \ldots \ldots$

5.12 Time- and azimuthally-averaged skin friction coefficient from CFUND 1, CFUND 2 and CFUND 3. . . . . . . . . . . . . . . . 151

5.13 Time-averaged heat transfer $(d T / d y)$ at the wall from CFUND 1, CFUND 2 and CFUND 3. The streamwise alligned "hot" streaks observed in our simulations looks qualitatively similar to the streamwise streaks observed in the Purdue experiments using temperature sensitive paints for a flared

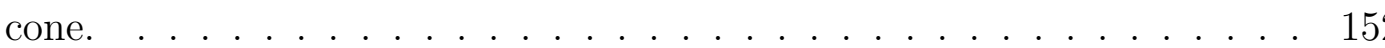

5.14 Time-averaged skin friction from CFUND 1, CFUND 2 and CFUND 3. The streamwise alligned "hot" streaks observed in our simulations looks qualitatively similar to the streamwise streaks observed in the Purdue experiments using temperature sensitive paints for a flared cone.

5.15 Visualization of flow structures by isosurface of Q criterion from CFUND 3. (a) Full view of the cone (top) and (b) close-up view of the last downstream part of the cone (bottom). The isosurface is colored using the streamwise velocity magnitude. . . . . . . . . . . . . . 154 


\section{LIST OF FIGURES-Continued}

5.16 Visualization of flow structures by isosurface of $\mathrm{Q}$ criterion. Shown are perspective view of isosurface of $\mathrm{Q}$ criterion from CFUND 1 (top left), CFUND 2 (top right) and CFUND 3 (bottom). The isosurface is colored using the streamwise velocity magnitude. . . . . . . . . . . 155

5.17 Visualization of flow structures by isosurface of $\mathrm{Q}$ criterion. Shown are close-up view of isosurface of Q criterion from CFUND 1 (top left), CFUND 2 (top right) and CFUND 3 (bottom). The isosurface is colored using the streamwise velocity magnitude. . . . . . . . . . .

5.18 Van Driest transformed streamwise velocity profiles normalized with the friction velocity for different streamwise positions from CFUND 1 (top left), CFUND 2 (top right) and CFUND 3 (bottom). For streamwise position close to the peak in the skin friction $\left(x^{*}=0.57 m\right)$, the van Driest transformed velocity approaches the theroretical curves. The dotted lines indicate the linear sublayer and the law of the wall. . . . . . . . . . . .

5.19 Downstream development of select mean-flow properties: (a) shape factor, $H_{12}$ (top) and (b) Reynolds number based on momentum thickness, $R_{\theta}$ (bottom) from CFUND 1, CFUND 2 and CFUND 3. The time average was obtained for five forcing periods $\left(T_{\text {forcing }}\right) \ldots \ldots \ldots$

5.20 Energy spectra versus $k_{\phi}$ for $u, v$ and $w$ from CFUND 1 (top left), CFUND 2 (top right) and CFUND 3 (bottom) at $x^{*}=0.57 m$. The red and blue lines indicate the theoretical estimate for the decay of the energy spectrum: $E \sim k_{\phi}^{-5 / 3}$ (red line) and $E \sim k_{\phi}^{-7}$ (blue line). . . . . . 161

$5.21 \mathrm{~N}$-factor curves for oblique waves with azimuthal wave number $k_{c}=20$. The N-factor curves were obtained from the low-amplitude wave packet simulation 


\section{LIST OF FIGURES-Continued}

5.22 Streamwise development of the maximum u-velocity disturbance amplitude from the oblique breakdown simulation. . . . . . . . . . . . . . . 164

5.23 Time and azimuthal averaged skin friction coefficient from the oblique breakdown simulation. Note that the interval for time average is indicated by the number of forcing periods. . . . . . . . . . . . . . . . 16

5.24 Time averaged heat transfer $(d T / d y)$ and skin friction at the wall from the oblique breakdown simulation. The streamwise aligned streaks observed in the case of oblique breakdown looks strikingly different from that observed for fundamental breakdown. . . . . . . . . . . . . . 166

5.25 Visualization of flow structures by isosurface of $\mathrm{Q}$ criterion from the oblique breakdown simulation. The isosurface is colored using the streamwise velocity magnitude. . . . . . . . . . . . . . . . . . 167

5.26 Close-up view of isosurface of $\mathrm{Q}$ criterion from the oblique breakdown simulation on the last downstream part of the cone (top) and at the late stage of transition (bottom). The isosurface is colored using the streamwise velocity magnitude. . . . . . . . . . . . . . . . . 168

5.27 Van Driest transformed streamwise velocity profiles normalized with the friction velocity for different streamwise positions from the oblique breakdown simulation. . . . . . . . . . . . . . . . . 169

5.28 Downstream development of select mean-flow properties: (a) shape factor, $H_{12}$ (top) and (b) Reynolds number based on momentum thickness, $R e_{\theta}$ (bottom) from the oblique breakdown simulation. Time average was obtained for five forcing periods $\left(T_{\text {forcing }}\right) \ldots \ldots$. . . . . . . . . . 170 


\section{LIST OF FIGURES-Continued}

5.29 Energy spectra versus $k_{\phi}$ for $u, v$ and $w$ at (a) $x^{*}=0.47 m$, (b) $x^{*}=0.49 m$ and (c) $x^{*}=0.53 \mathrm{~m}$. The red and blue lines indicate the theoretical estimate for the decay of the energy spectrum: $E \sim k_{\phi}^{-5 / 3}$ (red line) and $E \sim k_{\phi}^{-7}$ (blue line). . . . . . . . . . . . . . . . . 171 


\section{LIST OF TABLES}

3.1 Flow parameters from the sharp cone experiments conducted in the Boeing/AFOSR Mach 6 quiet tunnel at Purdue University. . . . . . . . . . . 53

5.1 Forcing parameters for the fundamental breakdown simulations. . . . . . 143 


\title{
NOMENCLATURE
}

\author{
Abbreviations \\ $2 D, 3 D$ Two-/three-dimensional \\ CFD Computational Fluid Dynamics \\ DNS Direct Numerical Simulations \\ LST Linear Stability Theory \\ TPS Thermal Protection System
}

\section{Greek}

$\alpha \quad$ Complex streamwise wave number

$\alpha_{i} \quad$ Streamwise amplification rate

$\alpha_{r} \quad$ Streamwise wave number

$\beta \quad$ Spanwise wave number

$\Delta t \quad$ Time step

$\delta \quad$ Boundary layer thickness

$\theta_{c} \quad$ Cone half angle

$\Theta \quad$ Phase

$\gamma \quad$ Ratio of specific heats

$\lambda_{z} \quad$ Azimuthal wave length

$\lambda_{x} \quad$ Streamwise wave length

$\mu \quad$ Dynamic viscosity

$\rho \quad$ Density

$\tau_{i j} \quad$ Viscous stresses

$\phi \quad$ Placeholder for different variables

$\omega \quad$ Angular frequency 


\section{Roman}

\begin{tabular}{|c|c|}
\hline$A$ & Disturbance amplitude \\
\hline$c$ & Speed of sound \\
\hline$c_{f}$ & Skin friction coefficient \\
\hline$c_{p}$ & Specific heat at constant pressure \\
\hline$c_{v}$ & Specific heat at constant volume \\
\hline $\mathbf{E}_{c}, \mathbf{F}_{c}$ & Convective flux terms \\
\hline $\mathbf{E}_{d}, \mathbf{F}_{d}$ & Dissipative flux terms \\
\hline$E_{t}$ & Total energy \\
\hline$F$ & Reduced frequency \\
\hline $\mathbf{H}$ & Source term \\
\hline$k$ & Thermal conductivity \\
\hline$k_{c}$ & Azimuthal mode number based on cone circumference \\
\hline$L$ & Reference length scale [m] \\
\hline$M$ & Mach number \\
\hline$p$ & Pressure \\
\hline $\operatorname{Pr}$ & Prandtl number \\
\hline $\mathbf{q}$ & Heat flux vector \\
\hline$Q$ & Q-criterion \\
\hline$R$ & Gas constant \\
\hline$R_{x}$ & Reynolds number based on boundary layer scaling \\
\hline$R e$ & Global Reynolds number based on free stream values \\
\hline$R e_{e}$ & Reynolds number based on boundary layer edge values \\
\hline$r$ & Radius of the cone \\
\hline$T$ & Temperature \\
\hline$T_{\text {forcing }}$ & Forcing period \\
\hline$t$ & Time \\
\hline
\end{tabular}


$u, v, w \quad$ Streamwise, wall-normal and azimuthal velocity components

$U \quad$ Vector of conservative variables

$x, y, \varphi \quad$ Streamwise, wall-normal and azimuthal coordinates

\section{Subscripts}

$\begin{array}{ll}\infty & \text { Free-stream value } \\ a w & \text { Adiabatic wall } \\ e & \text { Boundary layer edge conditions } \\ \max & \text { Maximum in wall-normal direction } \\ (n, k) & \text { Multiples of frequency, azimuthal wavenumber } \\ i & \text { Imaginary part } \\ r & \text { Real part } \\ w & \text { Wall value }\end{array}$

\section{Superscripts}

Reynolds-averaged flow quantity

* Dimensional quantities

$+\quad$ In near-wall units

, Disturbance quantities 


\begin{abstract}
Direct Numerical Simulations (DNS) are performed to investigate laminar-turbulent transition in a boundary layer on a sharp cone at Mach 6. The main objective of this dissertation research is to explore which nonlinear breakdown mechanisms may be dominant in a broad-band "natural" disturbance environment and then use this knowledge to perform controlled transition simulations to investigate these mechanisms in great detail. Towards this end, a "natural" transition scenario was modeled and investigated by generating wave packet disturbances. The evolution of a threedimensional wave packet in a boundary layer has typically been used as an idealized model for "natural" transition to turbulence, since it represents the impulse response of the boundary layer and, thus, includes the interactions between all frequencies and wave numbers. These wave packet simulations provided strong evidence for a possible presence of fundamental and subharmonic resonance mechanisms in the nonlinear transition regime. However, the fundamental resonance was much stronger than the subharmonic. In addition to these two resonance mechanisms, the wave packet simulations also indicated the possible presence of oblique breakdown mechanism.

To gain more insight into the nonlinear mechanisms, controlled transition simulations were performed of these mechanisms. Several small and medium scale simulations were performed to scan the parameter space for fundamental and subharmonic resonance. These simulations confirmed the findings of the wave packet simulations, namely that, fundamental resonance is much stronger compared to the subharmonic resonance. Subsequently a set of highly resolved fundamental and oblique breakdown simulations were performed. In these DNS, remarkable streamwise arranged "hot" streaks were observed for both fundamental and oblique breakdown. The streaks were a consequence of the large amplitude steady longitudinal vortex modes in the nonlinear regime. These simulations demonstrated that both second-mode fundamental breakdown and oblique breakdown may indeed be viable paths to complete breakdown to turbulence in hypersonic boundary layers at Mach 6 .
\end{abstract}




\section{INTRODUCTION}

\subsection{Motivation}

On February 24th 1949, the pens on the automatic plotting machine at South Station were busy tracking the altitude and course of a rocket that just moments before had been launched from a site close to the test range of the White Sands Proving Ground. The rocket was a $\mathrm{V}-2$, mounted on top of the $\mathrm{V}-2$ was a slender, needle-like rocket called the WAC Corporal, which was a second-stage motor to follow after the burnout of the $\mathrm{V}-2$. The $\mathrm{V}-2 / \mathrm{WAC}$ Corporal combination was the first attempt to implement a multistage rocket for achieving high velocities and high altitudes. The pen plotters tracked the rocket to an altitude of 100 miles at a velocity of 3500 miles per hour, at that point, the WAC Corporal was fired. As a result, the slender upper stage accelerated to a maximum velocity of 5150 miles per hour and reached an altitude of 244 miles, exceeding the previous record set by a V-2 by 130 miles. After reaching that peak altitude, the WAC Corporal nosed over and plunged back into the atmosphere at more than 5000 miles per hour. It became the first human-manufactured object to achieve hypersonic flight and the first time any vehicle has flown faster than five times the speed of sound (Anderson, 1997, 2006).

In 1961 on April 12th at 10:55 a.m. a strange spherical object landed under the canopy of a parachute. Its surface was charred black, and it had three small viewing ports covered with heat-resistant glass. Inside that capsule was Russian flight major Yuri Gagarin, only 108 minutes earlier he had been sitting on a rocket at the Russian comsodrome at Baikonur, near the Aral Sea. Gagarin's orbital craft, called Vostok I was slowed down at 10:25 a.m. by starting a retro-rocket and entered the atmosphere at a speed greater than 25 times the speed of sound. Thus, thirty minutes later, Gagarin had become the first man to fly in space, to orbit the earth, to experience hypersonic flight and to return safely (Anderson, 1997, 2006). 


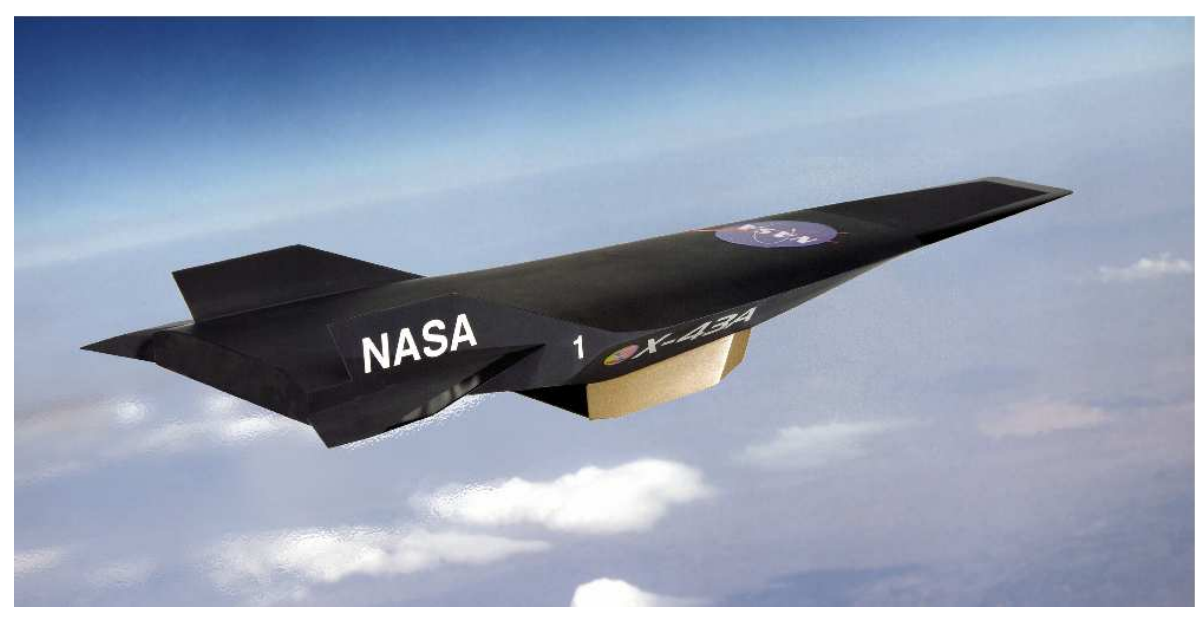

Figure 1.1: X-43A hypersonic research vehicle (Morelli et al., 2005).

In fact, year 1961 saw many more manned hypersonic flights. In May, Alan B. Shepard became the the second man and the first American to experience hypersonic flight when his capsule reentered the atmosphere at a speed above Mach 5 after a suborbital flight over the Atlantic Ocean. In June, U.S. Air Force test pilot Major Robert White then flew the $\mathrm{X}-15$ airplane at Mach 5.3, the first flight to exceed Mach 5 for the $\mathrm{X}-15$ airplane. Five months later White bettered his own record on November 9th by flying the X-15 at Mach 6 (Anderson, 1997). Thus, both unmanned and manned hypersonic flight, suddenly burst onto the aerodynamics community in the 1950s and 1960s without anyone having been prepared for it (Anderson, 2006). Hypersonic aerodynamics research was in somewhat of a catch-up mode right from the beginning. Despite the dearth of knowledge at that time about flows at hypersonic Mach numbers, there was little hesitation to send those flights at their incredible speeds. The advancements in hypersonic flight in the 20th century were highlighted by the space shuttle - the ultimate in manned airplanes with its Mach 26 re-entry into the Earth's atmosphere after the end of each mission in space. A recent success has been the X-43 Hyper-X unmanned research vehicle (see figure 1.1) that made history in November 2004 by achieving sustained flight at approximately Mach 10. It 
was the first sustained atmospheric hypersonic flight powered by a scramjet (Morelli et al., 2005; Peebles, 2008, 2011).

Hypersonic flow is physically different from low-speed flow (Anderson, 1997, 2006). It is characterized by new physical phenomena that become important at high Mach numbers. For example, the enormous amount of aerodynamic heating (due to the frictional effect of the flow over the surface) on the structure of the flight vehicles represents the main difficulty in the design and safe operation of hypersonic vehicles. It is indeed the limiting factor in aerodynamic designs for hypersonic vehicles. Most importantly, at large Reynolds numbers, these aero-thermal loads are further increased due to the transition of a laminar boundary layer to turbulence. Therefore, laminar-turbulent boundary layer transition has important design implications for any Thermal Protection System (TPS) that is needed to protect a hypersonic flight vehicle and its crew (e.g. the space shuttle).

In the past and even on recent hypersonic vehicles, engineers have used a rather conservative approach for designing the TPS. In these designs a turbulent boundary layer was assumed to be present over the entire surface (Berry \& Horvarth, 2007). Hence, appropriate measures to guard against the heat transfer due to these aerothermal loads are expensive and result in significant weight penalties. Accurate estimation of the laminar-turbulent transition region is of vital importance for designing future hypersonic vehicles, because only then can the aero-thermal loads and surface temperatures be adequately predicted, ultimately leading to enhanced payload capabilities. In addition to surface heating, transition to turbulence also has a significant effect on the aerodynamic performance of hypersonic flight vehicles, as the skin friction for turbulent boundary layers is considerably higher than for the laminar boundary layer (Schneider, 2004).

Laminar-Turbulent transition in hypersonic boundary layers is a major unresolved topic in fluid dynamics. Even after many years of research, crucial aspects of the transition physics are still in the dark. A major difficulty here is the increased com- 
plexity of the transition physics due to the compressibility effects. The lack of a basic understanding of hypersonic transition, and, as a consequence, a lack of reliable transition prediction tools, hinders all practical applications discussed above, e.g., reusable launch vehicles, high-speed interceptors, high-speed civil transport (HSCT), high-speed reconnaissance aircrafts, Falcon hypersonic technology vehicle (HTV) and airbreathing hypersonic cruise vehicles (see, Lin et al., 1984; Schneider, 2004, 2006). Hypersonic transition research suffers from the fact that reliable experiments are very difficult and expensive. Wind tunnel noise level (see, Schneider, 2001), short run times and controlled disturbance generation are only a few of the difficulties faced in experiments. Therefore, relatively few reliable experimental studies of the growth of instability waves have been conducted. On the other hand, Direct Numerical Simulations (DNS) of transition in hypersonic boundary layers require large compute times and high grid resolution. As a consequence, most of the earlier research efforts for hypersonic transition have focused on the receptivity (the process by which free-stream disturbances generate instability waves in the boundary layer) and the linear regime of boundary layer transition (e.g. Demetriades, 1960, 1977; Kendall, 1975; Stetson et al., 1983; Maslov et al., 2001; Zhong, 2001). Very little is known regarding the nonlinear stages of transition and in particular, regarding the final breakdown to turbulence. However, due to the stabilizing effect of compressibility, the nonlinear transition regime extends over a significant downstream extent of a hypersonic flight vehicle. As a result, transition prediction tools based on the linear $\mathrm{N}$-factor calculations cannot estimate the location where the flow becomes turbulent accurately. Therefore, new transition prediction tools, which incorporate nonlinear transition physics are desperately needed for the development and safe operation of future highspeed flight vehicles. Hence, it is very important to understand the nonlinear stages of transition, including the final strongly nonlinear breakdown, in order to identify which disturbance scenarios lead to turbulent flow and which do not. Therefore, accurate and reliable fully resolved DNS of hypersonic boundary layers are essential 
for advancing our understanding of hypersonic transition and for the development of reliable transition prediction tools.

The motivation for the present research is to make a contribution towards the understanding of the nonlinear stages of transition and of the final breakdown to turbulence in hypersonic boundary layers.

\subsection{Laminar-Turbulent Transition in Boundary Layers}

One of the most important characteristics of a fluid is the flow state in which it is moving, e.g. laminar, turbulent or the transitional state in between. Laminar flow is a well predictable, structured and layered flow (lamina in latin means: layer, sheet or leaf), which usually results in significantly less frictional resistance to solid bodies and much less mixing than the chaotic, swirly and unsteady state of the fluid in a turbulent flow (Schlatter, 2005). The evolution of an initially laminar flow into a fully developed turbulent flow is called laminar-turbulent transition. The most important non-dimensional parameter that determines whether a flow will be laminar or turbulent is the Reynolds number $R e$, which is the ratio between the inertial and viscous forces acting on the fluid particles. At low Re the flows are laminar, however, it becomes unstable to disturbances at some finite critical Reynolds number. Then a sequence of linear and nonlinear higher order instabilities leads to transition to the fully developed turbulent state.

Laminar-Turbulent transition is a multifold process that evolves in many different paths depending on numerous parameters of the mean flow and disturbances. However, it is possible to identify the main paths to transition (Morkovin et al., 1994; Reshotko, 2008; Fedorov, 2011; Zhong \& Wang, 2012) and these paths are schematically shown in figure 1.2. Path A indicates transition in a weak disturbance environment and consists of three stages: receptivity, linear eigenmode growth, and nonlinear breakdown. The receptivity process converts the external free-stream 


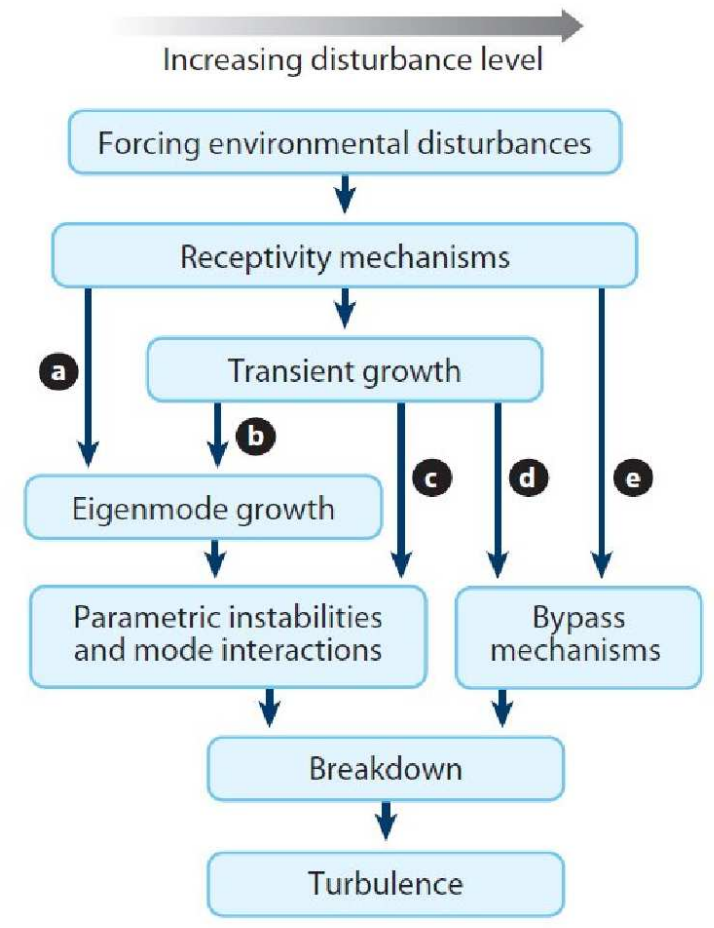

Figure 1.2: Laminar-Turbulent transition paths in boundary layers. Path A: three stage transition process in a weak disturbance environment; path B: three stage transition process with weak transient growth; paths $\mathrm{C}$ and $\mathrm{D}$ : transition process with strong transient growth; path E: bypass transition due to large amplitude free-stream disturbances. Figure taken from Fedorov (2011). 
disturbance into boundary layer instability waves (see Goldstein \& Hultgren, 1989; Maslov et al., 2001; Saric et al., 2002; Ma \& Zhong, 2003; Fedorov, 2003; Zhong \& Ma, 2006) and provides their initial conditions e.g. amplitudes, frequencies, and phases. The linear modal growth of the instability waves can be obtained by solving the eigenvalue problem posed by the homogeneous linearized stability equations. The final breakdown to turbulence is caused by the nonlinear secondary and higher instabilities when the boundary layer instability waves reach certain finite amplitudes. As the free-stream disturbance amplitude increases, transient growth mechanisms that arise from the nonorthogonal nature of the Orr-Sommerfeld and Squire eigenfunctions become important. A weak transient growth provides a higher initial amplitude to the eigenmodes (path B). Whereas, a strong transient growth can directly lead to strong nonlinear interactions or breakdown to turbulence (path $\mathrm{C}$ and $\mathrm{D}$ ). In fact, for very large amplitude disturbances there is no linear regime (path E). Thou, these transition paths are the same for both low- and high-speed boundary layers, the basic features may be different for these two cases. Note that, in this dissertation research, transition in boundary layers on a flat plate and a sharp cone at zero angle of attack in a low-disturbance environment is investigated. Therefore, path $\mathrm{A}$ is the most relevant to the research work discussed here.

Even for simple geometries like a flat plate or a sharp cone at zero angle of attack, the extension of subsonic and supersonic stability theories and transition prediction methods to hypersonic flows is not straightforward (Fedorov, 2011). The physics of high-speed boundary layer transition are much more complex than for low-speeds. From linear stability theory (Mack, 1969, 1975, 1984), it is known that multiple instability modes exist for high-speed boundary layer flows, in contrast to only one mode (Tollmien-Schlichting, TS) for the incompressible case. In addition to the so-called first mode in supersonic/hypersonic boundary layers, higher modes exist for supersonic/hypersonic boundary layers that result from an inviscid instability mechanism. According to linear stability theory (LST), the most unstable of the higher modes is 
the second mode. In contrast to the first modes, which have the highest amplification rates for oblique wave angles, the second modes have the highest amplification for two-dimensional waves. Also from LST, it is known that the first mode is dominant (higher amplification rates) for low supersonic Mach numbers while for Mach numbers above 4 (hypersonic boundary layers) the second mode is dominant. Due to the difficulties in carrying out experiments (and "controlled" experiments, in particular) and due to the existence of multiple instability modes, the role and importance of the various instability modes in a realistic transition process are not understood at all. Of course, when amplitudes of the various instability modes reach high enough levels, nonlinear interactions of these modes can occur. As a consequence, the transition process in hypersonic boundary layers is highly non-unique, which means that slight changes in the disturbance environment or vehicle geometry may significantly alter the transition process.

\subsection{Flow Past a Circular Sharp Cone at Zero Angle of Attack}

Cones with circular cross section represent a useful prototypical geometry for investigating boundary layer stability and transition at high-speeds (Schneider, 2004). In fact, in most of the modern high-speed applications, the nose region of the flight vehicle can be approximated as some sort of a cone. A visual example of the laminarturbulent transition on a sharp cone is shown in figure 1.3, which shows a portion of a shadowgraph obtained in the Naval Ordnance Lab ballistics range (Reda, 1979). The sharp cone model shown in figure 1.3 is at near zero angle of attack and the cone is traveling from left to right through still air. The boundary layer on the lower surface is turbulent and acoustic waves radiating from the turbulent eddies can be seen traveling downstream at the Mach angle. The boundary layer on the upper surface, however, is only intermittently turbulent, with two turbulent spots visible in figure 1.3. Stronger waves can be seen in front of these turbulent spots and weaker 


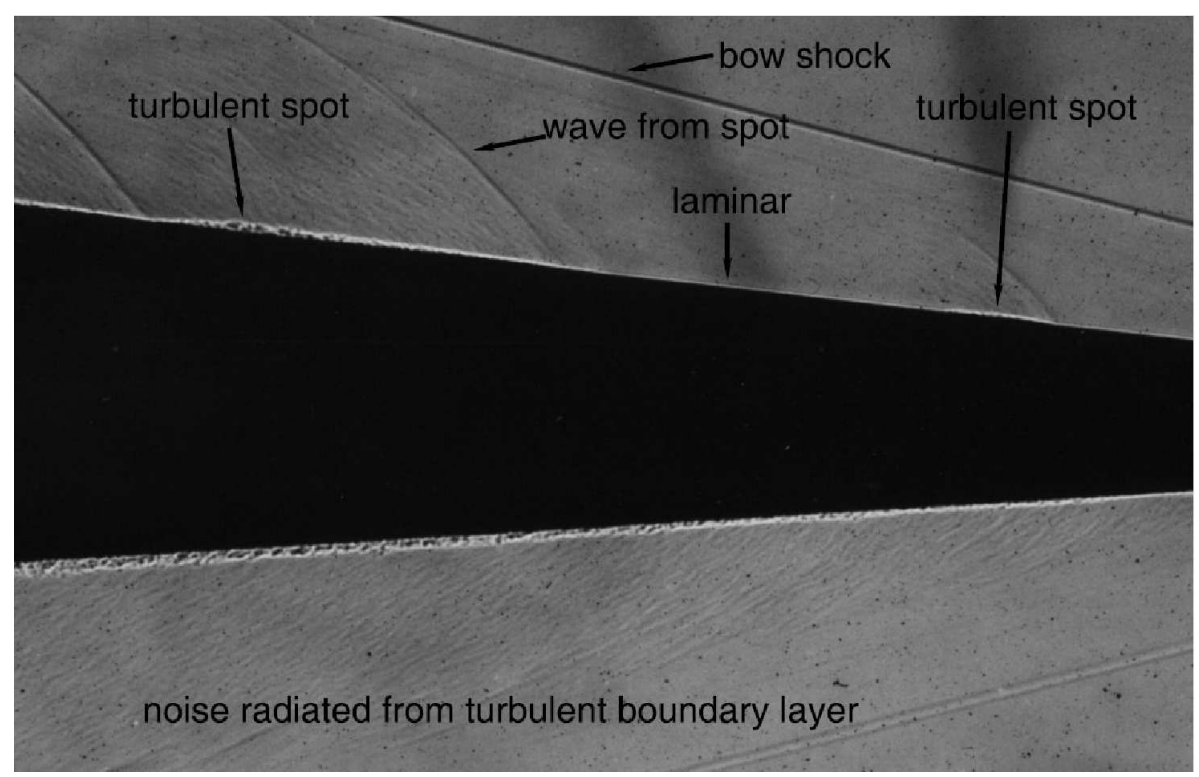

Figure 1.3: Shadowgraph of transition on a sharp cone at Mach 4.31 (Reda, 1979). The image is from Shot 6728, the cone model is at near zero angle of attack and the length of the 5 degree half-angle cone is 9.144 inches. The cone is traveling from left to right through still air. Picture taken from Schneider (2004).

acoustic waves can be seen radiating from the turbulence within the spot. Note that these acoustic waves are not present in the laminar regions.

The most detailed experiments for hypersonic boundary layers on a circular cone are those of Stetson et al. (1983, 1984, 1985, 1986) and Stetson \& Kimmel (1992, 1993). They have investigated the influence of nose radius, unit reynolds number, and transverse curvature for a cone boundary layer at Mach 8 in a conventional "noisy" wind tunnel. In all cases, they found that the two-dimensional or axisymmetric second-mode waves were the dominant instability waves. However, from these experiments it was still not clear what role the second-mode waves play in the nonlinear stages of the transition process. In recent experiments, Shiplyuk et al. (2003) and Bountin et al. (2008) investigated transition in a sharp cone boundary layer at Mach 6 in a conventional tunnel. They perturbed the boundary layer using glow discharge actuator as a harmonic point source. Using the bi-coherence spectrum 
method they concluded that the basic mechanism of nonlinear interaction at the location of the maximum r.m.s. voltage fluctuation in a Mach 6 cone boundary layer was a subharmonic resonance.

Some of the first linear stability calculations for a sharp-cone boundary layer were conducted by Malik (1984), Mack (1987), and Gasperas (1987). Malik (1984) investigated a supersonic boundary layer on a sharp cone ( $5^{\circ}$ half angle) at zero angle of attack for various Mach numbers. He did not consider the curvature terms for the calculation of the mean-flow profiles and for the eigenvalue analysis. The meanflow conditions for his calculations matched the flight experiments from Fisher \& Dougherty (1982) (Mach 1.2, 1.35, 1.6 and 1.92) and the quiet tunnel experiments by Beckwith et al. (1983) for Mach 3.5 (before shock). Malik (1984) showed that in these low free-stream disturbance experiments, transition could be predicted by the $e^{N}$ method with $\mathrm{N}$ ranging from 9 to 11 . This was confirmed by the experimental and numerical studies by Chen et al. $(1988,1989)$ for a sharp cone and a flat plate at Mach 3.5. Mack (1987) and Gasperas (1987) investigated the linear stability behavior of disturbances in hypersonic boundary layers on sharp cones at Mach 8 and compared their results to the Stetson experiments (Stetson et al., 1983). Results of Mack (1987) and Gasperas (1987) obtained from linear stability theory did not agree well with the experiments. This could be due to the nonlinear effects in the experiments.

Many numerical investigations have addressed the transition problem for the flow conditions of the Stetson experiments (Pruett et al., 1995; Pruett \& Chang, 1995; Fezer \& Kloker, 2004; Husmeier \& Fasel, 2007). But even today, the nonlinear transition regime of hypersonic cone boundary layers is not understood.

\subsection{Scope of Present Research}

So far, most of the transition investigations for supersonic/hypersonic boundary layers have focused on particular transition routes that include a few specified instability 
waves. For example, Thumm (1991) and Fasel et al. (1993) found the so-called "oblique breakdown" for a boundary layer at Mach 1.6. This breakdown mechanism is initiated by the nonlinear interaction of two oblique instability waves with equal but opposite wave angles (see, e.g., Adams \& Kleiser, 1993; Sandham et al., 1995; Eissler \& Bestek, 1996; Fezer \& Kloker, 1999; Husmeier et al., 2005; Mayer et al., 2007, 2008, 2009). Kosinov and co-workers (Kosinov et al., 1994b, a; Ermolaev et al., 1996; Kosinov et al., 1997) investigated the nonlinear transition regime of a supersonic boundary layer and reported a different breakdown mechanism, which they called "asymmetric subharmonic resonance." In this resonance mechanism, one oblique fundamental wave interacts with two oblique (asymmetric) subharmonic waves. Corke et al. (2002) performed controlled experiments for a sharp cone at Mach 3.5 ( $7^{\circ}$ half angle) at NASA Langley and reported "asymmetric subharmonic resonance" where the fundamental and subharmonic waves have the same wave angle. In recent experiments, Shiplyuk et al. (2003) and Bountin et al. (2008) investigated transition in a sharp cone boundary layer at Mach 6 in a conventional noisy tunnel. They perturbed the boundary layer using glow discharge actuator as a harmonic point source. Using the bi-coherence spectrum method they concluded that the basic mechanism of nonlinear interaction at the location of the maximum r.m.s. voltage fluctuation in a Mach 6 cone boundary layer was a subharmonic resonance.

Experimental and numerical investigations of controlled transition are very important and play a vital role in validating theoretical results for both the linear and the nonlinear transition regimes. However, they can only provide limited insight into a natural transition scenario where a broad disturbance spectrum is excited by free-stream turbulence, and, as a consequence of this, complex wave interactions between all disturbance modes are possible. Therefore, the objective of the present research work is to first determine which nonlinear mechanisms may be dominant in a broad-band disturbance environment ("natural" transition scenario) and then use this knowledge to perform controlled transition simulations for investigating these 
mechanisms in great detail. Towards this end, a broad-band "natural" transition scenario was modeled and investigated using wave packet disturbances, which were generated by a short-duration (localized) blowing and suction pulse. These wave packets contains a wide range of disturbance frequencies and wave numbers.

In the past, this approach was widely used to model and investigate natural transition for incompressible boundary layers. The work of Gaster \& Grant (1975) was one of the first detailed experiments to document the structure of the wave packet as it evolved. By placing a hot-wire probe just outside the boundary layer at the second maximum of the linear eigenfunction for the streamwise disturbance velocity they were able to study the growth of the disturbances. Their measurements compared very well to the results computed from the theoretical model developed by Gaster (1975), which treated the wave packet as a summation over all frequencies and spanwise wavenumbers of the least stable linear eigenmodes. By computing the amplitude of each mode as it travelled downstream, Gaster (1975) was able to reproduce both the growth and structure of the low-amplitude (linear) wave packet. At the last measuring station in the experiments by Gaster \& Grant (1975) the smooth structure of the wave packet appeared to be breaking down and they theorized that this might have been due to nonlinear effects. This was confirmed by the measurements of Cohen et al. (1991) who extended the work of Gaster \& Grant (1975) and measured the vertical structure of the disturbance through the boundary layer and followed the evolution of the wave packet through the nonlinear regime. Later, Cohen (1994) extended Gaster's theoretical model and compared the amplitude evolution of the two- and three-dimensional disturbances measured by Cohen et al. (1991) with an extended linear theory. Then Medeiros \& Gaster $(1999 a, b)$ extended the famous Gaster \& Grant (1975) experiments and observed that during the initial linear evolution, the form of disturbances was insensitive to the initial phase of their excitation pulse. However, the development of nonlinear effects depended markedly on the initial phase of their imposed disturbance. Direct Navier-Stokes 
computations have also been used to study wave packets in incompressible boundary layers. The numerical simulations by Konzelmann (1990) and Konzelmann \& Fasel (1991) closely reproduced Gaster \& Grant's original experiment. Recently, Forgoston \& Tumin $(2005,2006)$ formulated an initial-value problem for three-dimensional wave packets in compressible boundary layers. The problem is solved using a Laplace transform with respect to time and Fourier transforms with respect to the streamwise and spanwise coordinates. They presented the solution as a sum of modes consisting of continuous and discrete spectra of temporal stability theory.

The laminar-turbulent transition process can proceed in a variety of ways (see, Morkovin et al., 1994; Fedorov, 2011) depending on numerous parameters of the mean flow and disturbances. The path studied in this research involves the development of linearly unstable waves that grow and excite three-dimensional secondary and higherorder instabilities. This would lead to the development of regions of large shear away from the wall, and breakdown into small-scale turbulence. Hence, here the developing wave packet would eventually lead to sporadic patches of turbulence in an otherwise laminar surrounding flow. Emmons (1951) was the first to characterize the patches of turbulence and call them turbulent spots (for an incompressible boundary layer). He discovered turbulent spots when observing a thin layer of water flowing down an inclined plate. As the turbulent spots appeared in bursts on the lower surface they could be visualized by the disturbance they caused on the water surface. He correctly anticipated that spots would appear in other wall-bounded flows also, like in channel flows (see, Carlson et al., 1982; Alavyoon et al., 1986; Henningson et al., 1987; Henningson \& Kim, 1991) and even in pipes where they have been called puffs. (see, Wygnanski \& Champagne, 1973; Shan et al., 1999) Spots are isolated regions of large fluctuations within a laminar shear flow. The fluctuations within the spot have properties that are close to those of a turbulent flow. They travel downstream, grow in size, and coalesce with neighboring spots to form extended regions of turbulence. In general, turbulent spots are a characteristic feature of transition to turbulence in 
an environment with high amplitude free-stream disturbances. (Jacobs \& Durbin, 2001)

Most of the earlier investigations on turbulent spots have also been for incompressible flows (see, Wygnanski et al., 1976, 1982; Narasimha, 1985). These spots have a characteristic arrowhead shape and have a lateral (spreading) half-angle growth rate, which is a constant, at least for simple boundary layers. For incompressible flow with zero-pressure-gradient, the lateral spreading angle is approximately 10 degrees (Schubauer \& Klebanoff, 1956). Many other characteristics of turbulent spots in incompressible (low-speed) flows have been well documented in the literature (see, Wygnanski et al., 1979; Katz et al., 1982; Gad-el Hak et al., 1981; Glezer et al., 1989; Seifert \& Wygnanski, 1995). The lateral spreading or growth of the turbulent spot strongly depends on the Mach number. Fischer (1972) showed that increasing the free-stream Mach number results in a reduction of lateral spreading by a factor of 3 or 4. Due to the difficulties in performing good experiments for high-Mach-number flows, there are very few studies on the effect of compressibility. Only recently, DNS of the growth of turbulent spot in supersonic boundary layers at Mach 2, 4 and 6 were performed by Krishnan \& Sandham $(2006 a, c)$. Their spots showed the typical structures seen in the incompressible case. Krishnan \& Sandham (2006a) investigated the effect of Mach number (compressibility) on the spot characteristics and confirmed the stabilizing effect of the Mach number. In addition, they also investigated the various aspects of turbulent spot growth mechanisms, spot merging (Krishnan \& Sandham, $2006 b)$ and the interference of the spot with a shock-induced separation bubble (Krishnan \& Sandham, 2007). Even more recently, Jocksch \& Kleiser (2008) performed DNS to investigate the growth of isolated turbulent spots in laminar zero-pressuregradient boundary layers on a flat-plate at Mach 1.1 and 5. Redford et al. (2011) investigated the effect of the Mach number and wall temperature on the lateral growth of turbulent spots in supersonic boundary layers with free-stream Mach numbers of 3 and 6. Note that in the present research, where small-amplitude disturbances 
are introduced, the growth of primary instability waves starts the transition process. Whereas, in the studies by Krishnan \& Sandham (2006a,c), Jocksch \& Kleiser (2008) and Redford et al. (2011) the introduction of large-amplitude perturbations causes a bypassing of the linear stages of transition. They chose an initial disturbance amplitude strong enough to cause a rapid development into a turbulent spot. Thereby, the breakdown to turbulence occurs before the linear and weakly nonlinear disturbance development occurred in the computational domain. Thus the sequence of events leading to a mature turbulent spot may be different if the spot develops from a small-amplitude pulse. For the present investigation, flow parameters from the experiments by Schneider (2009) in the Boeing/AFOSR Mach 6 quiet tunnel at Purdue university are used. 


\section{GOVERNING EQUATIONS AND NUMERICAL METHODS}

\subsection{Governing Equations}

The physical problem under consideration is governed by the three-dimensional compressible Navier-Stokes equations, consisting of conservation of mass, momentum and energy. The flow is assumed to be an ideal gas with constant specific heat coefficients. The Prandtl number is assumed to be constant and the flow is treated as a Newtonian fluid. In the following, the subscript $\infty$ refers to free-stream values and the asterisk * labels dimensional quantities. The governing equations are non-dimensionalized with a reference length scale $L^{*}$ and the freestream values of streamwise velocity $U_{\infty}^{*}$, temperature $T_{\infty}^{*}$, density $\rho_{\infty}^{*}$, viscosity $\mu_{\infty}^{*}$ and thermal conductivity $k_{\infty}^{*}$.

The non-dimensional variables are defined as:

$$
\begin{gathered}
x=\frac{x^{*}}{L^{*}}, y=\frac{y^{*}}{L^{*}}, r=\frac{r^{*}}{L^{*}}, t=\frac{t^{*}}{L^{*} / U_{\infty}^{*}} \\
u=\frac{u^{*}}{U_{\infty}^{*}}, v=\frac{v^{*}}{U_{\infty}^{*}}, w=\frac{w^{*}}{U_{\infty}^{*}} \\
\rho=\frac{\rho^{*}}{\rho_{\infty}^{*}}, p=\frac{p^{*}}{\rho_{\infty}^{*} U_{\infty}^{* 2}}, T=\frac{T^{*}}{T_{\infty}^{*}} \\
\mu=\frac{\mu^{*}}{\mu_{\infty}^{*}}, k=\frac{k^{*}}{k_{\infty}^{*}}
\end{gathered}
$$

The non-dimensional compressible Navier-Stokes equations in conservative variable formulation written in conical coordinates are,

$$
\frac{\partial \boldsymbol{U}}{\partial t}+\frac{1}{r} \frac{\partial\left(r\left(\boldsymbol{E}_{\boldsymbol{c}}+\boldsymbol{E}_{\boldsymbol{d}}\right)\right)}{\partial x}+\frac{1}{r} \frac{\partial\left(r\left(\boldsymbol{F}_{\boldsymbol{c}}+\boldsymbol{F}_{\boldsymbol{d}}\right)\right)}{\partial y}+\frac{1}{r} \frac{\partial\left(\boldsymbol{G}_{\boldsymbol{c}}+\boldsymbol{G}_{\boldsymbol{d}}\right)}{\partial \varphi}+\boldsymbol{H}=0 .
$$

where $x$ is the downstream coordinate parallel to the cone surface, $y$ is the wallnormal coordinate orthogonal to the cone surface and $\varphi$ specifies the azimuthal coordinate. The radius $r$ denotes the local radius and is computed according to $r=$ 
$x \sin \theta_{c}+y \cos \theta_{c}$, where $\theta_{c}$ is the half angle (also called semi-vertex angle) of the cone. The vector of the conservative variables is given as $\boldsymbol{U}=\left[\rho, \rho u, \rho v, \rho w, E_{t}\right]$. The convective flux vectors $\left(\boldsymbol{E}_{\boldsymbol{c}}, \boldsymbol{F}_{\boldsymbol{c}}, \boldsymbol{G}_{\boldsymbol{c}}\right)$, the viscous and heat conduction terms $\left(\boldsymbol{E}_{\boldsymbol{d}}, \boldsymbol{F}_{\boldsymbol{d}}, \boldsymbol{G}_{\boldsymbol{d}}\right)$ and the source term $(\boldsymbol{H})$ are

$$
\begin{aligned}
& \boldsymbol{E}_{\boldsymbol{c}}=\left[\begin{array}{c}
\rho u \\
\rho u^{2}+p \\
\rho u v \\
\rho u w \\
\left(E_{t}+p\right) u
\end{array}\right], \boldsymbol{F}_{\boldsymbol{c}}=\left[\begin{array}{c}
\rho v \\
\rho u v \\
\rho v^{2}+p \\
\rho v w \\
\left(E_{t}+p\right) v
\end{array}\right], \boldsymbol{G}_{\boldsymbol{c}}=\left[\begin{array}{c}
\rho w \\
\rho u w \\
\rho v w \\
\rho w^{2}+p \\
\left(E_{t}+p\right) w
\end{array}\right] \\
& \boldsymbol{E}_{\boldsymbol{d}}=\left[\begin{array}{c}
0 \\
-\tau_{x x} \\
-\tau_{x y} \\
-\tau_{x \varphi} \\
-u \tau_{x x}-v \tau_{x y}-w \tau_{x \varphi}+q_{x}
\end{array}\right], \boldsymbol{F}_{\boldsymbol{d}}=\left[\begin{array}{c}
0 \\
-\tau_{x y} \\
-\tau_{y y} \\
-\tau_{y \varphi} \\
-u \tau_{x y}-v \tau_{y y}-w \tau_{y \varphi}+q_{y}
\end{array}\right], \\
& \boldsymbol{G}_{\boldsymbol{d}}=\left[\begin{array}{c}
0 \\
-\tau_{x \varphi} \\
-\tau_{y \varphi} \\
-\tau_{\varphi \varphi} \\
-u \tau_{x \varphi}-v \tau_{y \varphi}-w \tau_{\varphi \varphi}+q_{\varphi}
\end{array}\right] \\
& \boldsymbol{H}=\left[\begin{array}{c}
0 \\
-\frac{1}{r} \sin \theta_{c}\left(\rho w^{2}-\tau_{\varphi \varphi}+p\right) \\
-\frac{1}{r} \cos \theta_{c}\left(\rho w^{2}-\tau_{\varphi \varphi}+p\right) \\
\frac{1}{r} \sin \theta_{c}\left(\rho u w-\tau_{x \varphi}\right)+\frac{1}{r} \cos \theta_{c}\left(\rho v w-\tau_{y \varphi}\right) \\
0
\end{array}\right] .
\end{aligned}
$$

The total energy is computed as

$$
E_{t}=\rho\left(\frac{T}{(\gamma-1) \gamma M^{2}}+\frac{1}{2}\left(u^{2}+v^{2}+w^{2}\right)\right) .
$$

The stresses in the conical coordinate system are determined by

$$
\begin{aligned}
\tau_{x x} & =\frac{\mu}{R e}\left(\frac{4}{3} \frac{\partial u}{\partial x}-\frac{2}{3} \frac{\partial v}{\partial y}-\frac{2}{3} \frac{1}{r} \frac{\partial w}{\partial \varphi}-\frac{2}{3} \frac{1}{r}\left(u \sin \theta_{c}+v \cos \theta_{c}\right)\right) \\
\tau_{y y} & =\frac{\mu}{R e}\left(\frac{4}{3} \frac{\partial v}{\partial y}-\frac{2}{3} \frac{\partial u}{\partial x}-\frac{2}{3} \frac{1}{r} \frac{\partial w}{\partial \varphi}-\frac{2}{3} \frac{1}{r}\left(u \sin \theta_{c}+v \cos \theta_{c}\right)\right) \\
\tau_{\varphi \varphi} & =\frac{\mu}{R e}\left(\frac{4}{3} \frac{1}{r} \frac{\partial w}{\partial \varphi}-\frac{2}{3} \frac{\partial u}{\partial x}-\frac{2}{3} \frac{\partial v}{\partial y}+\frac{4}{3} \frac{1}{r}\left(u \sin \theta_{c}+v \cos \theta_{c}\right)\right)
\end{aligned}
$$




$$
\begin{gathered}
\tau_{x y}=\frac{\mu}{R e}\left(\frac{\partial v}{\partial x}+\frac{\partial u}{\partial y}\right), \\
\tau_{x \varphi}=\frac{\mu}{R e}\left(\frac{1}{r} \frac{\partial u}{\partial \varphi}+\frac{\partial w}{\partial x}-\frac{1}{r} w \sin \theta_{c}\right), \\
\tau_{y \varphi}=\frac{\mu}{R e}\left(\frac{1}{r} \frac{\partial v}{\partial \varphi}+\frac{\partial w}{\partial y}-\frac{1}{r} w \cos \theta_{c}\right),
\end{gathered}
$$

and the heat flux vector is given as

$$
\boldsymbol{q}=-\frac{\mu}{(\gamma-1) \operatorname{RePr} M^{2}}\left(\frac{\partial T}{\partial x}, \frac{\partial T}{\partial y}, \frac{1}{r} \frac{\partial T}{\partial \varphi}\right)
$$

The set of equation is closed with the equation of state for ideal gas,

$$
p=\frac{1}{\gamma M^{2}} \rho T
$$

and the viscosity is calculated using sutherland's law with low temperature correction (Mack, 1965) as follows

$$
\mu= \begin{cases}\frac{C_{1}}{\mu_{\infty}^{*}} T_{1}^{*} & T^{*}<T_{1}^{*} \\ \frac{C_{1}}{\mu_{\infty}^{*}}\left(T T_{\infty}^{*}\right) & T_{1}^{*} \leq T^{*} \leq T_{2}^{*} \\ \frac{C_{2}}{\mu_{\infty}^{*}}\left(T T_{\infty}^{*}\right)^{3 / 2} /\left(T T_{\infty}^{*}+T_{2}^{*}\right) & T^{*}>T_{2}^{*}\end{cases}
$$

with

$$
\begin{array}{r}
C_{1}=6.93873 \cdot 10^{-8} \mathrm{Ns} / \mathrm{m}^{2} \mathrm{~K}, \quad T_{1}^{*}=40 \mathrm{~K} \\
C_{2}=1.4580 \cdot 10^{-6} \mathrm{Ns} / \mathrm{m}^{2} \mathrm{~K}^{1 / 2}, \quad T_{2}^{*}=110.4 K .
\end{array}
$$

Furthermore, the nondimensional parameters in the above equations are defined as

$$
R e=\frac{\rho_{\infty}^{*} U_{\infty}^{*} L^{*}}{\mu_{\infty}^{*}}, \quad \operatorname{Pr}=\frac{\mu_{\infty}^{*} c_{p \infty}^{*}}{k^{*}} \quad \text { and } \quad M=\frac{U_{\infty}^{*}}{c_{\infty}^{*}}=\frac{U_{\infty}^{*}}{\sqrt{\gamma p_{\infty}^{*} / \rho_{\infty}^{*}}}
$$

with $c, k, c_{p}$ being the speed of sound, the thermal conductivity and the specific heat at constant pressure, respectively. 


\subsection{Time Integration}

The Navier-Stokes equations are integrated in time using a standard explicit fourthorder Runge-Kutta method (Ferziger, 1998). Applied to the following model ordinary differential equation

$$
\frac{d \phi}{d t}=f(t, \phi)
$$

the explicit fourth-order Runge-Kutta method can be written as

\section{1st substep:}

$$
\phi_{1}:=\phi_{n}+\frac{\Delta t}{2} f\left(t, \phi_{n}\right)
$$

2nd substep:

$$
\begin{aligned}
\phi_{2} & :=\phi_{n}+\frac{\Delta t}{2} f\left(t+\frac{\Delta t}{2}, \phi_{1}\right), \\
\phi_{1} & :=\phi_{1}+2 \phi_{2},
\end{aligned}
$$

\section{3rd substep:}

$$
\begin{aligned}
\phi_{2} & :=\phi_{n}+\Delta t f\left(t+\frac{\Delta t}{2}, \phi_{2}\right), \\
\phi_{1} & :=\frac{1}{3}\left(-\phi_{n}+\phi_{1}+\phi_{2}\right),
\end{aligned}
$$

4th substep:

$$
\phi_{n+1}:=\phi_{1}+\frac{\Delta t}{6} f\left(t+\Delta t, \phi_{2}\right) .
$$

where $\phi$ is a placeholder for the conservative variables and $\Delta t$ is the stepsize in time. The symbol ":=" in equations (2.16) indicates that these equations are assignments and thus are only valid in the context of a programming language. Variables with the subscript $n$ are from the old timestep $n$ while variables with subscript $n+1$ are the result of the time integration (new timestep). This scheme combines accuracy and robustness and was extensively applied by Harris (1997), Meitz \& Fasel (2000), Sandberg (2004) and Mayer (2009). 


\subsection{Spatial Discretization}

The spatial discretization is based on high-order accurate finite differences. In particular, the derivatives of the viscous terms $\left(\boldsymbol{E}_{\boldsymbol{d}}, \boldsymbol{F}_{\boldsymbol{d}}, \boldsymbol{G}_{\boldsymbol{d}}\right)$ and the source term $(\boldsymbol{H})$ are discretized using $6^{\text {th }}$-order non-compact central finite differences in the streamwise direction and $4^{t h}$-order central finite differences in the wall-normal direction. In the azimuthal direction, a pseudo-spectral discretization using Fast Fourier Transforms is employed. The inviscid fluxes $\left(\boldsymbol{E}_{\boldsymbol{c}}, \boldsymbol{F}_{\boldsymbol{c}}, \boldsymbol{G}_{\boldsymbol{c}}\right)$ are separated into an upwind flux and a downwind flux using van Leer's splitting (van Leer, 1982). Then, grid centered upwind differences (Zhong, 1998) with $9^{\text {th }}$-order accuracy are applied to evaluate the derivatives for these fluxes. These grid centered upwind differences are derived using the factor $\alpha$, which prescribes the degree of upwinding,

$$
\frac{\partial \phi_{i}}{\partial x}=\sum_{k=i-N}^{i+N} c_{k} \phi_{k}-\alpha \overline{\Delta x} \frac{\partial^{2 N-1} \phi_{i}}{\partial x^{2 N-1}} .
$$

Hereby $\phi_{i}$ denotes the flow variable at the grid point $i$, the $c_{k}$ 's are the stencil coefficients and $\overline{\Delta x}$ is the averaged grid spacing over the stencil interval. The parameter $N$ determines the number of grid points in the stencil. For example the $9^{\text {th }}$-order upwind scheme is derived by setting $N=5$ and $\alpha=-1500$. Note that for $\alpha=0$ the upwind scheme reduces to a central difference scheme. All stencil coefficients are derived on a stretched grid.

Special attention is given to the wall-next boundary stencils. Since high-order accurate boundary closures are typically unstable, the present code is adopting a method suggested by Zhong \& Tatineni (2003). The main idea is to stabilize the numerical scheme by using a stretched grid close to the wall. In particular, the following stretching function is applied:

$$
y_{j}=\frac{\arcsin \left(-\alpha \cos \left(\pi j / 2 n_{y}\right)\right)}{\arcsin (\alpha)} .
$$

This formula is a modification of the original formula, i.e. stretching is only applied 
at the wall boundary and not (as in Zhong \& Tatineni (2003)) both at the wall and free-stream boundary.

This high-order accurate finite difference code was developed in our CFD Laboratory to be used for supersonic/hypersonic transition research. For a more detailed description of the code see Laible et al. (2008, 2009)

\subsection{Boundary Conditions}

\subsubsection{Inflow Boundary Condition}

The inflow boundary for hypersonic boundary layer simulations is typically split into two regions: a subsonic region $(M<1)$ close to the wall and a supersonic/hypersonic region $(M>1)$ away from the wall. In the supersonic/hypersonic region, Dirichlet conditions for $u, v, w, T, p$ and $\rho$ are specified, which are obtained from a precursor calculation. For the subsonic region in the boundary layer, oftentimes a special treatment is necessary to avoid undesired reflections, e.g. the non-reflecting boundary condition suggested by Poinsot \& Lele (1992). However, the simulations presented here did not show any evidence of reflections at the inflow boundary, even when the Dirichlet conditions were also applied for the subsonic region. Therefore, for all the simulations presented in this dissertation Dirichlet conditions were prescribed at the inflow over the entire domain height.

\subsubsection{Wall Boundary Condition}

On the cone surface, no-penetration $(v=0)$ and no-slip $(u=0, w=0)$ conditions are enforced. Adiabatic and isothermal wall (with $T_{w}^{*}=300 \mathrm{~K}$ ) boundary conditions are used for the steady base-flow calculations and temperature fluctuations are assumed to vanish for the unsteady simulations. The value of the pressure at the wall boundary is obtained from the $y$-momentum equation. Finally, density is computed using the equation of state (equation 2.11). 


\subsubsection{Outflow Boundary Condition}

At the outflow, the second derivative of the primitive variables are set to zero: $\partial^{2} u / \partial x^{2}=0, \partial^{2} v / \partial x^{2}=0, \partial^{2} w / \partial x^{2}=0, \partial^{2} T / \partial x^{2}=0, \partial^{2} p / \partial x^{2}=0$. The density is then determined from the temperature and the pressure by using the equation of state (equation 2.11). For simulations into the nonlinear transition regime a buffer domain technique is applied, where finite amplitude disturbances are ramped to zero at the outflow (see, Meitz \& Fasel, 2000).

\subsubsection{Free-stream Boundary Condition}

For all the simulations presented in this thesis, the free-stream boundary is located above the oblique shock emanating from the nose of the cone. Therefore, Dirichlet conditions $(u, v, w, T, p, \rho$ prescribed) can be prescribed at this boundary. Positioning the free-stream boundary above the oblique shock wave was found to improve the quality of the simulation results when compared with an earlier approach where we positioned the free stream boundary below the oblique shock and prescribed nonreflecting boundary conditions.

\subsection{Disturbance Generation}

\subsection{1 pulse forcing}

In the wave packet simulations discussed in chapter 4, localized pulse disturbances are introduced in a circular area on the cone surface by pulsing the wall-normal velocity with the streamwise and spanwise distributions as shown in figure 2.1a. The time signal of the pulse is plotted in figure $2.1 \mathrm{~b}$. The duration of the pulse is about $0.5 \%$ of the total simulation time. In dimensional form, this value corresponds to about $0.00345 \mathrm{~ms}$ while the entire simulation time is about $0.68645 \mathrm{~ms}$. 

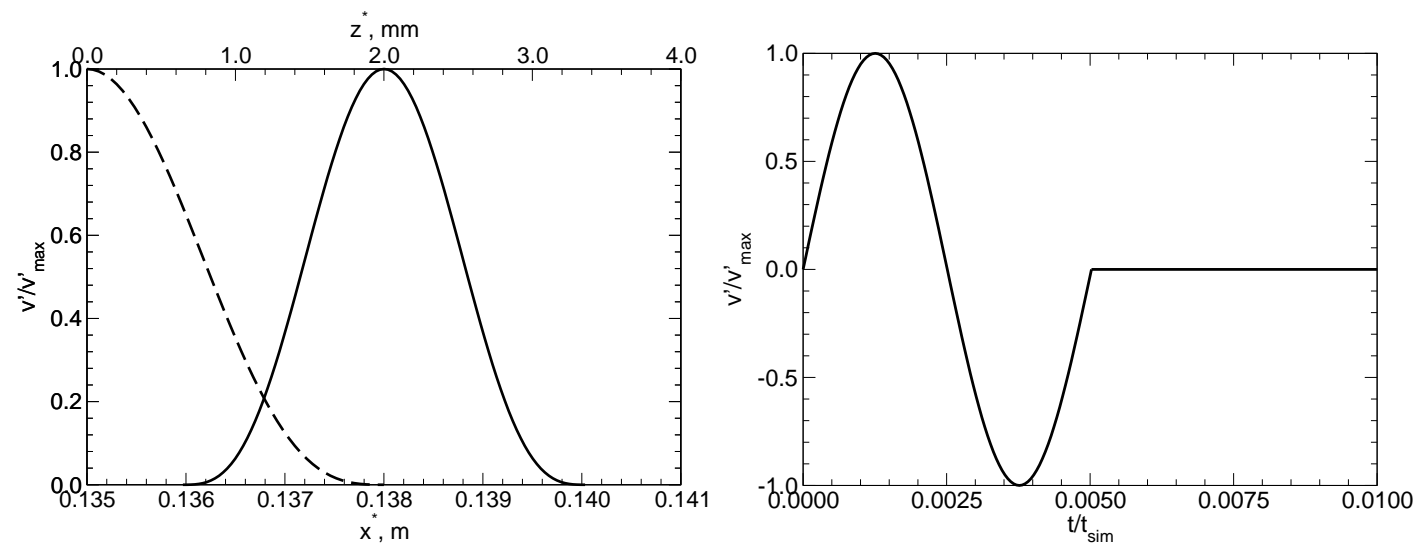

Figure 2.1: Wall-normal velocity at the wall (a) as a function of streamwise and azimuthal direction (left) and (b) as a function of time (right). In (a), the dashed line (- -) represents the azimuthal distribution and the solid line $(-)$ represents the streamwise distribution of the wall-normal velocity.

The forcing signal can be obtained from

$$
v\left(x_{h}, y=0, z_{h}, t\right)=\left\{\begin{aligned}
A \cos ^{3}\left(\pi x_{h}\right) \cos ^{3}\left(\pi z_{h}\right) \sin (-\omega t), & t<t_{1} \\
0, & t \geqslant t_{1}
\end{aligned}\right.
$$

where dimensionless coordinates $x_{h}$ and $z_{h}$ are defined as

$$
x_{h}=\frac{x-0.5\left(x_{2}+x_{1}\right)}{x_{2}-x_{1}} \quad \text { and } \quad z_{h}=\frac{z}{z_{2}-z_{1}}
$$

respectively. $A$ denotes the forcing amplitude and $\omega$ is the forcing frequency. As can be seen in figure 2.1, the forcing starts at $x_{1}^{*}=0.136 \mathrm{~m}$ in streamwise direction and ends at $x_{2}^{*}=0.140 m$, in the spanwsise direction it extends from $z_{1}^{*}=-0.002 m$ to $z_{2}^{*}=0.002 m$, thus resulting in a circular area of $4 \mathrm{~mm}$ diameter.

\subsection{2 harmonic forcing}

In the controlled transition simulations discussed in Chapter 5, the disturbances are introduced into the flow by time harmonic wall-normal blowing and suction through a disturbance slot near the upstream end of the computational domain. The nopenetration boundary condition for the wall normal velocity component is replaced 


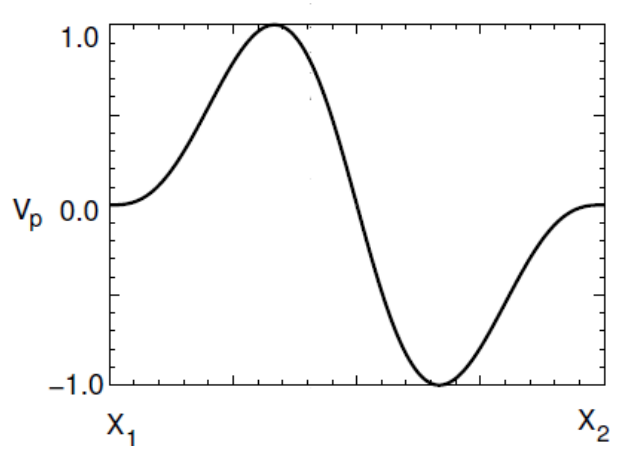

Figure 2.2: Schematic of the streamwise wall normal velocity disturbance distribution in the forcing slot used for introducing controlled disturbances.

at the blowing and suction slot by

$$
v(x, y=0, \varphi, t)=A_{\omega, k_{c}} v_{p}\left(x_{p}\right) \cos \left(k_{c} \varphi\right) \sin (\omega t)
$$

where $A_{\omega, k_{c}}, \omega$ and $k_{c}$ denote the amplitude, frequency and azimuthal wave number of a disturbance. The dimensionless coordinate $x_{p}$ is defined over the slot width $\left(x_{1} \leq x \leq x_{2}\right)$ by

$$
x_{p}=\frac{2 x-\left(x_{2}+x_{1}\right)}{x_{2}-x_{1}}
$$

where $x_{1}^{*}=0.136 m$ and $x_{2}^{*}=0.140 m$.

The wall normal velocity disturbance distribution $\left(v_{p}\right)$ in the streamwise direction represents a dipole modeled by a fifth-order polynomial, which is smooth everywhere including the endpoints (Harris, 1997) and is shown schematically in figure 2.2. 


\section{AXISYMMETRIC CONE BASE FLOW}

\subsection{Flow Parameters and Physical Problem}

The flow parameters used for the simulations presented in this thesis are based on the experimental conditions of the Boeing/AFOSR Mach 6 Quiet Tunnel (BAM6QT)at Purdue university (Schneider, 2008, 2009). The BAM6QT can be operated as a conventional noisy tunnel or as a quiet tunnel and it is currently one of the two hypersonic quiet tunnels in the world. It features low noise of about $0.05 \%$, which is similar to flight and an order of magnitude lower than conventional tunnels. The tunnel is a Ludwieg Tube - a long pressurized tube with a converging-diverging nozzle at the end. The flow passes from the driver tube, through the test section, diffuser, and finally to the vacuum tank. The flow is initiated by bursting a double diaphragm that is located downstream of the diffuser. When the flow begins, an expansion wave travels upstream and then reflects between the upstream end of the driver tube and the contraction. Run times of $3-5$ seconds under quiet flow conditions are typical at present (see also, Rufer \& Schneider, 2006; Juliano et al., 2008).

A summary of the flow parameters used for the simulations is provided in table 3.1. These flow parameters represent the current peak quiet performance of the BAM6QT. The approach flow has a Mach number of 6 and a unit Reynolds number

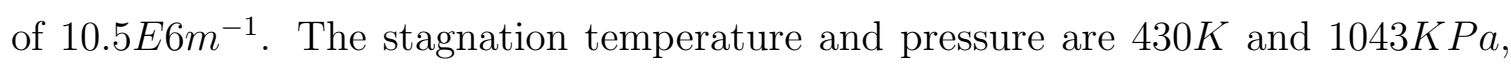
respectively.

The physical problem is a hypersonic boundary layer over a sharp circular cone investigated in the BAM6QT. The cone model used in the Purdue experiments has a semi vertex angle of $\theta_{c}=7^{\circ}$, and a cone length of $0.5 \mathrm{~m}$. The nose radius of the cone is $r_{\text {nose }}^{*}=0.05 \mathrm{~mm}$ and therefore, the cone can be considered as "a sharp cone." 


\begin{tabular}{|c|c|c|}
\hline \multicolumn{3}{|c|}{ Flow Parameters: } \\
\hline \multicolumn{3}{|c|}{ Approach flow : } \\
\hline$\overline{R e} / L$ & {$\left[m^{-1}\right]$} & $10.5 \cdot 10^{6}$ \\
\hline$M$ & $-]$ & 6.0 \\
\hline$T_{0}^{*}$ & $K]$ & 430.0 \\
\hline$p_{0}^{*}$ & $K P a]$ & 1043.0 \\
\hline \multicolumn{3}{|c|}{ Boundary layer edge : } \\
\hline$\overline{R e_{e} / L}$ & {$\left[m^{-1}\right]$} & $1313.6 \cdot 10^{6}$ \\
\hline$M_{e}$ & {$[-]$} & 5.35 \\
\hline$T_{e}^{*}$ & $K]$ & 63.9232 \\
\hline
\end{tabular}

Table 3.1: Flow parameters from the sharp cone experiments conducted in the Boeing/AFOSR Mach 6 quiet tunnel at Purdue University (Schneider, 2008, 2009).

\subsection{Simulation Strategy}

The basic flow for the circular cone boundary layer is computed using a high-orderaccurate finite difference code which was developed in our CFD Laboratory at the University of Arizona (Laible et al., 2008). The initial and boundary conditions are obtained from a precursor calculation using an in-house-developed finite-volume code (Gross \& Fasel, 2008). The entire procedure to obtain an accurate basic flow solution for the conical geometry is explained in detail in Laible et al. (2008). In figure 3.1 and 3.2, streamwise velocity and temperature profiles obtained from the high-order-accurate finite-difference code and the precursor calculation using the finite-volume code are compared to Mangler-transformed profiles calculated from a flat-plate similarity profile. Profiles at four different streamwise locations are shown for both adiabatic and isothermal wall, and at each of them the profiles show excellent agreement. Note that although the finite volume code predicts accurate boundary layer profiles, due to the lower order of accuracy, it is too diffusive to directly perform stability and transition simulations and would therefore require a very large number of grid cells/points.

A comparison of the boundary-layer thickness between the sharp cone basic flow 

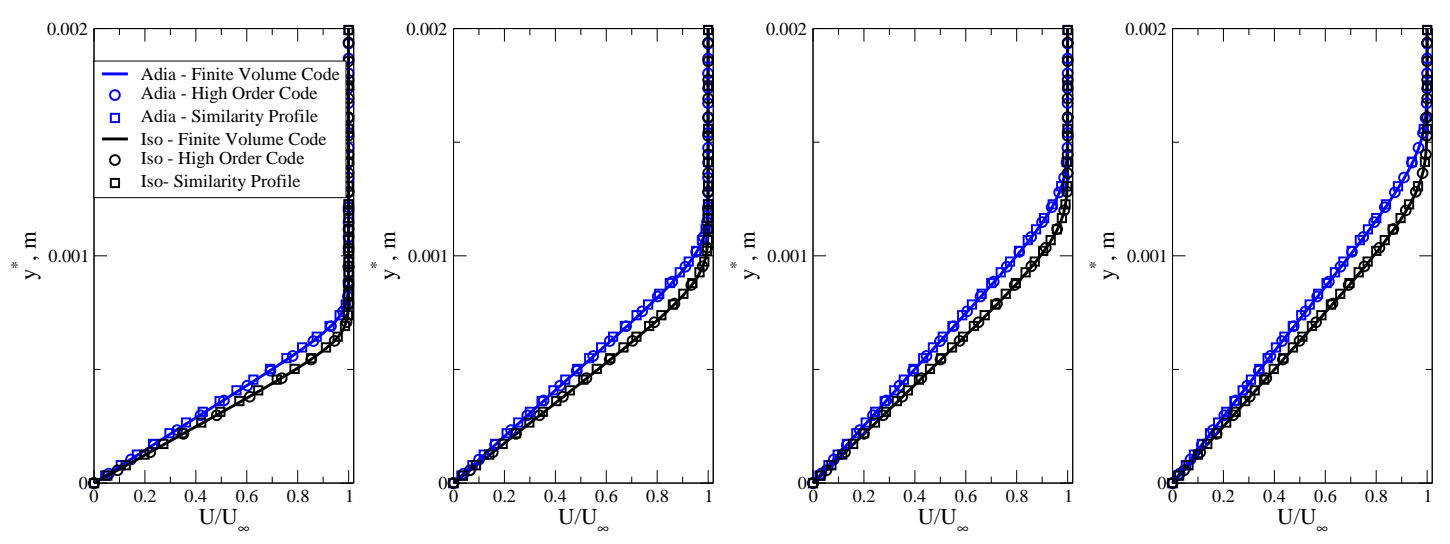

Figure 3.1: Comparison of streamwise velocity profiles computed by the precursor finite-volume code, the high-order code and the Mangler transformed flat plate similarity profile at the following streamwise locations: $x^{*}=0.100 m, x^{*}=0.200 m$, $x^{*}=0.300 m$ and $x^{*}=0.400 m$ (from left to right).
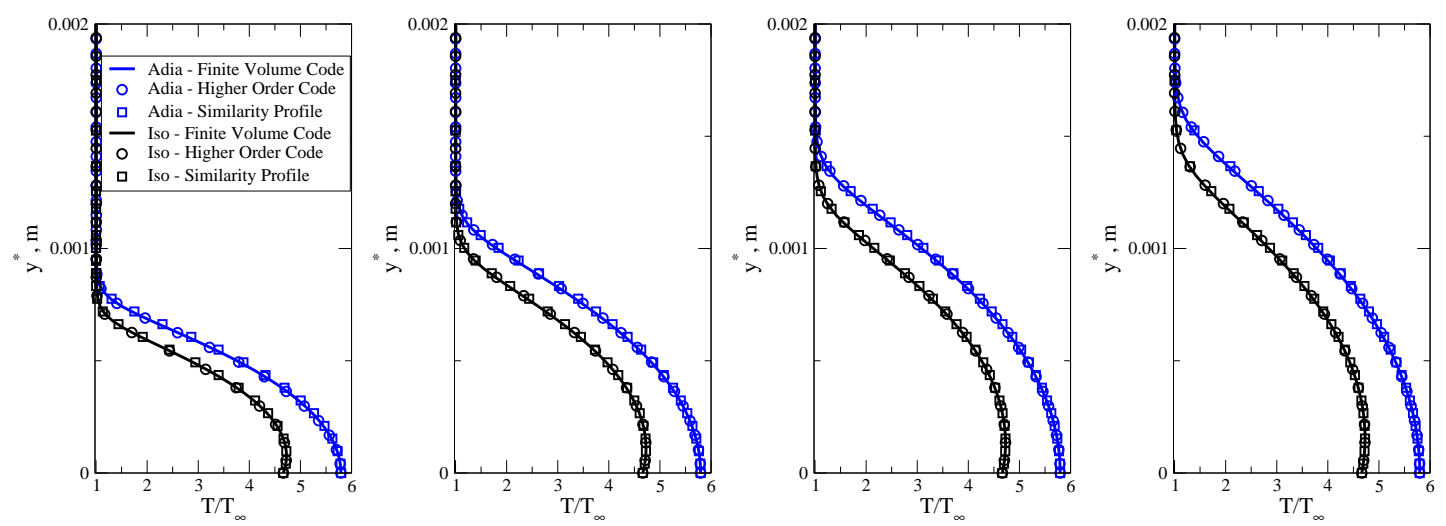

Figure 3.2: Comparison of temperature profiles computed by the precursor finitevolume code, the high-order code and the Mangler transformed flat plate similarity profile at the following streamwise locations: $x^{*}=0.100 m, x^{*}=0.200 m, x^{*}=0.300 m$ and $x^{*}=0.400 m$ (from left to right). 


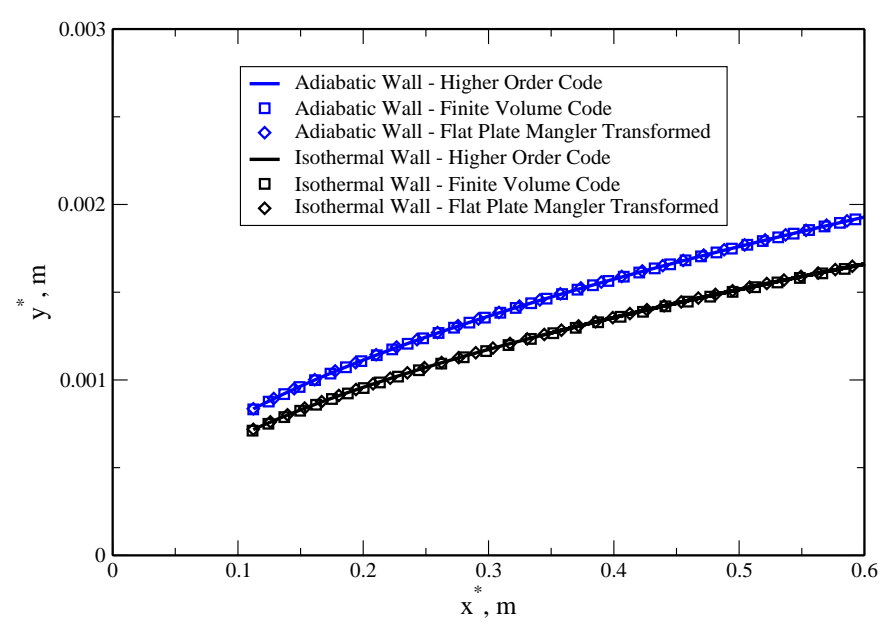

Figure 3.3: Comparison of the boundary layer thickness between the cone base flow simulations and a Mangler transformed flat plate similarity profile.

solution computed with the high-order code and a Mangler-transformed flat-plate similarity solution is shown in figure 3.3. Again, there is very good agreement. This agreement can be considered a validation of the basic flow calculation. Note that the boundary layer on the isothermal wall is thinner than the one on the adiabatic wall.

In order to be able to make a comparison with results from linear stability theory, the edge values for the cone boundary layer are determined as in Laible et al. (2008). The edge values are not constant in the streamwise direction, however the change is very small. Therefore, the edge values were assumed to be constant in the streamwise direction in order to simplify the post-processing. The boundary-layer-edge Mach number is $M_{e}=5.35$ and the unit Reynolds number is $R e_{e}=13.6 \mathrm{E} \mathrm{m}^{-1}$ for both adiabatic and isothermal wall. 


\section{THREE DIMENSIONAL WAVE PACKET SIMULATIONS}

Transition initiated by a three-dimensional wave packet is discussed in detail in this chapter for a sharp cone boundary layer at Mach 6. For hypersonic flows, it is still unclear what breakdown mechanism is most dominant and would transition a laminar boundary layer in a natural broad-band disturbance environment. The evolution of a three-dimensional wave packet in a boundary layer has often been used as an idealized model for natural, or unforced, transition to turbulence since it represents the impulse response of the boundary layer and thus includes the interactions between all frequencies and spanwise or azimuthal wave numbers. Hence, to provide some understanding of the natural transition scenario and to identify the dominant nonlinear breakdown mechanisms for hypersonic boundary layers, the growth and breakdown of a wave packet into a turbulent spot is investigated. Towards this end, several highly resolved DNS were performed and the results from these simulations are presented in this chapter. In order to generate a wave packet, a localized (short duration) threedimensional pulse disturbance is introduced into the boundary layer. This chapter starts with the discussion of a low-amplitude localized pulse disturbance simulation performed to investigate the linear development of a wave packet and then discusses simulations with increased amplitudes performed to investigate the weakly nonlinear and the late strongly nonlinear transition regimes.

\subsection{Simulation Setup}

The simulation setup follows the experimental study by Schneider and co-workers (Casper et al., 2009; Alba et al., 2010) in the Boeing/AFOSR Mach 6 Quiet Tunnel at Purdue University. They are currently conducting measurements of surface pressure fluctuations on a 7 degree sharp circular cone. In particular, they are measuring 
(a)

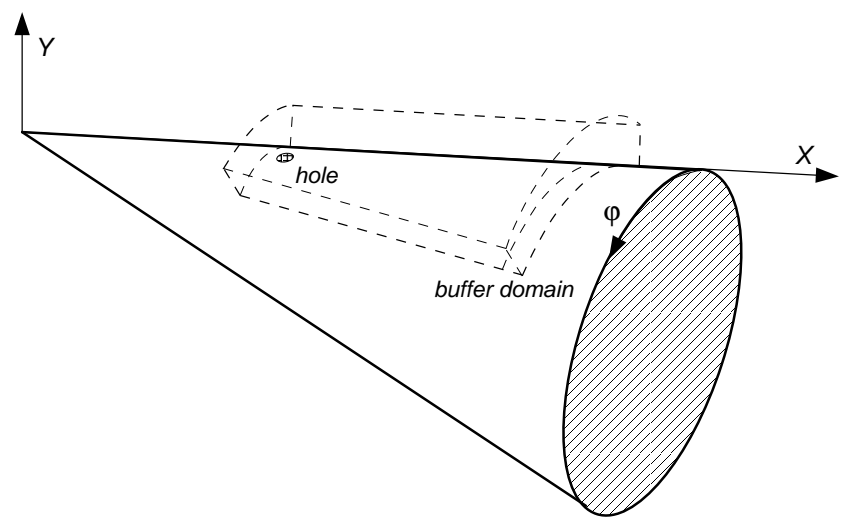

(b)

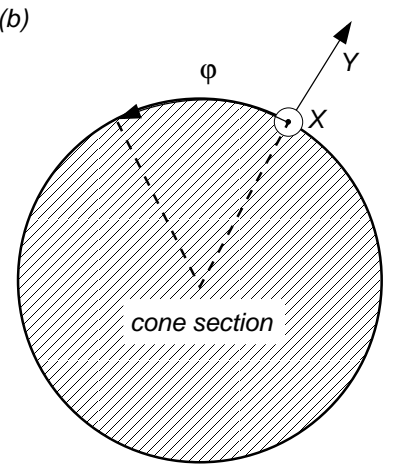

Figure 4.1: Cone model (a) with the computational domain and (b) cross section. The 'sharp' cone has a nose radius of $0.05 \mathrm{~mm}$ and a semi vertex angle of $7^{\circ}$.

pressure fluctuations beneath both naturally occurring instability wave packets and turbulent spots and the ones that are generated using spark and glow perturbers. While the glow perturber can only create discrete disturbances at a given frequency, the spark perturber can create an impulse with a broad-band frequency. Thus, the spark perturber experiments are the most relevant to the wave packet simulations discussed in this chapter.

A schematic of the computational domain and the corresponding coordinate system is shown in figure 4.1. The computational domain for all simulations starts at $x_{0}^{*}=0.1 m$ and, thus, does not include the nose tip. The outflow is positioned at $x_{L}^{*}=0.6 \mathrm{~m}$. A domain longer that the cone model used in the experiments is used to accommodate the buffer domain. The domain height is chosen as $y_{H}^{*}=0.123 \mathrm{~m}$. In the azimuthal direction, only one-third of the cone circumference is computed.

BAM6QT has a very short run time under quiet flow operation. The cone model remains cold at a constant temperature close to the room temperature. Therefore, an isothermal wall is the most appropriate boundary condition for the simulations (Schneider, 2009). In conventional tunnels, however, the run time is much longer and sometimes the cone model is preheated to bring it close to adiabatic wall temperature conditions (Bountin et al., 2008). Hence, two wall boundary conditions are used in the 
simulations to compare and study the effect of wall temperature under the BAM6QT flow conditions. An isothermal wall boundary condition with $T_{w}=300 \mathrm{~K}$ (Schneider, 2009) and an adiabatic wall $\left(T_{w}=T_{a w}\right)$ boundary condition are considered.

\subsection{Linear Wave Packet}

The linear transition regime for the cone is more complex than for the flat-plate geometry. For the cone, the spanwise curvature and the wave angle of a given disturbance (with a fixed azimuthal mode number) decrease in the downstream direction. The decreasing wave angle is a consequence of the body divergence of the cone geometry causing the azimuthal wave length of a given wave to increase in the downstream direction. The azimuthal wave length is defined as:

$$
\lambda_{z}=\frac{2 \pi r(x)}{k_{c}}
$$

at the streamwise position, $x$. Note that $k_{c}$ represents the azimuthal mode number. In the equation above, it is assumed that the ratio between boundary layer thickness and cone radius is small, hence the azimuthal wavelength is considered equal to the arc length on the cone surface. In this dissertation, a wave is labeled with a certain reduced frequency, $F$, and azimuthal mode number, $k_{c}$. For example, azimuthal mode number $k_{c}=1$ has an azimuthal wavelength corresponding to the circumference of the cone cross section, a wave with azimuthal mode number $k_{c}=2$ has an azimuthal wavelength corresponding to half the circumference, etc. Note that for a cone, the azimuthal mode number, $k_{c}$, has to be an integer and, consequently, only a discrete set of physically possible azimuthal wave lengths can exist. Whereas for a flat plate the spanwise wave length can, in principle, take any value in a continuous fashion. The reduced frequency, $F$, and the local Reynolds number, $R_{x}$, are defined as

$$
F=2 \pi \frac{f^{*} \nu_{e}^{*}}{U_{e}^{* 2}} \quad \text { and } \quad R_{x}=\sqrt{\frac{U_{e}^{*} x^{*}}{\nu_{e}^{*}}}
$$


respectively. Here, subscript, $e$, indicates the boundary layer edge values.

The linear stability regime of the cone boundary layer was investigated by introducing a localized three-dimensional pulse disturbance with a sufficiently small amplitude. An amplitude of $0.001 \%$ of the free-stream velocity was used to make sure that the packet developed linearly over the whole streamwise extent of the computational domain. In this research, the localized forcing was accomplished by a short-duration blowing and suction through a hole on the cone surface using the wall-normal velocity component. The pulse disturbance then develops into a threedimensional wave packet, which propagates downstream.

\subsubsection{Development of the Wave Packet}

The temporal and spatial development of the centerline wall-pressure disturbance amplitude is shown in figure 4.2 and 4.3 for the isothermal and adiabatic wall cases. Shown are time signals of the wave packet, which are plotted for several streamwise positions. This is also a convenient way of plotting the wave packet for experimentalists, since the measurements are made only at a few streamwise positions along the centerline. These figures provide an impression of the developments in the center region of the wave packet. Envelopes of these time signals, computed using Fourier transformations according to Gaster \& Grant (1975), are also included in figures 4.2 and 4.3. For both isothermal and adiabatic wall cases, it can be observed that the wave packet spreads and its amplitude increases as it propagates downstream. In these figures, a scale given with the signal on the bottom indicates the disturbance amplitude. It can be observed that the scale for the isothermal wall case is higher than the one for the adiabatic wall case. The waves within the wave packet in the case of the isothermal wall has reached larger amplitudes in comparison to the waves within the adiabatic wall wave packet. This suggests that the wall temperature has significant effects on the growth rate of disturbance waves. Typically, cooling the 
surface (isothermal wall or cold wall case) destabilizes the second-mode waves and stabilizes the first-mode waves. Surface cooling modifies the velocity and temperature profiles in the boundary layer and changes the stability characteristics of the boundary layer. The destabilized second-mode waves in the case of isothermal wall grows at a higher rate. As a result the envelope of the isothermal wall wave packet reaches a higher amplitude than the envelope of adiabatic wall wave packet.

The initial response of the flow to forcing through a hole is illustrated in figures 4.4 and 4.5 by contours of the instantaneous wall-pressure disturbance near the forcing location for several time instances for the isothermal and adiabatic wall cases. In figures $4.4 \mathrm{a}$ and $4.5 \mathrm{a}$ circular waves can be seen emanating outward from the hole. Initially, the wave packet has a completely three-dimensional structure, but as it propagates downstream the wave packet becomes less three-dimensional and the effects of stronger axisymmetric or two-dimensional waves can be observed for both the isothermal and adiabatic wall cases. This is expected because according to the linear stability theory at this Mach number, axisymmetric or two-dimensional (secondmode) waves experience higher streamwise amplification rates than do oblique waves. Both isothermal and adiabatic wall boundary layers respond similarly to the pulse disturbance.

Three snapshots of the wave packet for later time instances are shown in figures 4.6 and 4.7 for the two cases. Figures $4.6 \mathrm{a}$ and $4.7 \mathrm{a}$ are for early stages of its development. Three-dimensional waves (curved wave fronts) are seen to develop and lead the main wave packet as it propagates downstream. These waves are clearly visible in figures $4.6 \mathrm{~b}$ and $4.7 \mathrm{~b}$ and seem to travel faster than the main wave packet. Therefore, these waves could be the stable fast-mode waves or acoustic waves. It can be observed that the wave fronts within the main wave packet are predominantly axisymmetric. In addition to the axisymmetric waves, oblique waves can also be observed on the lateral sides of the packet. However, the amplitudes of these oblique waves are small compared to those of the dominant axisymmetric waves. These oblique waves are 


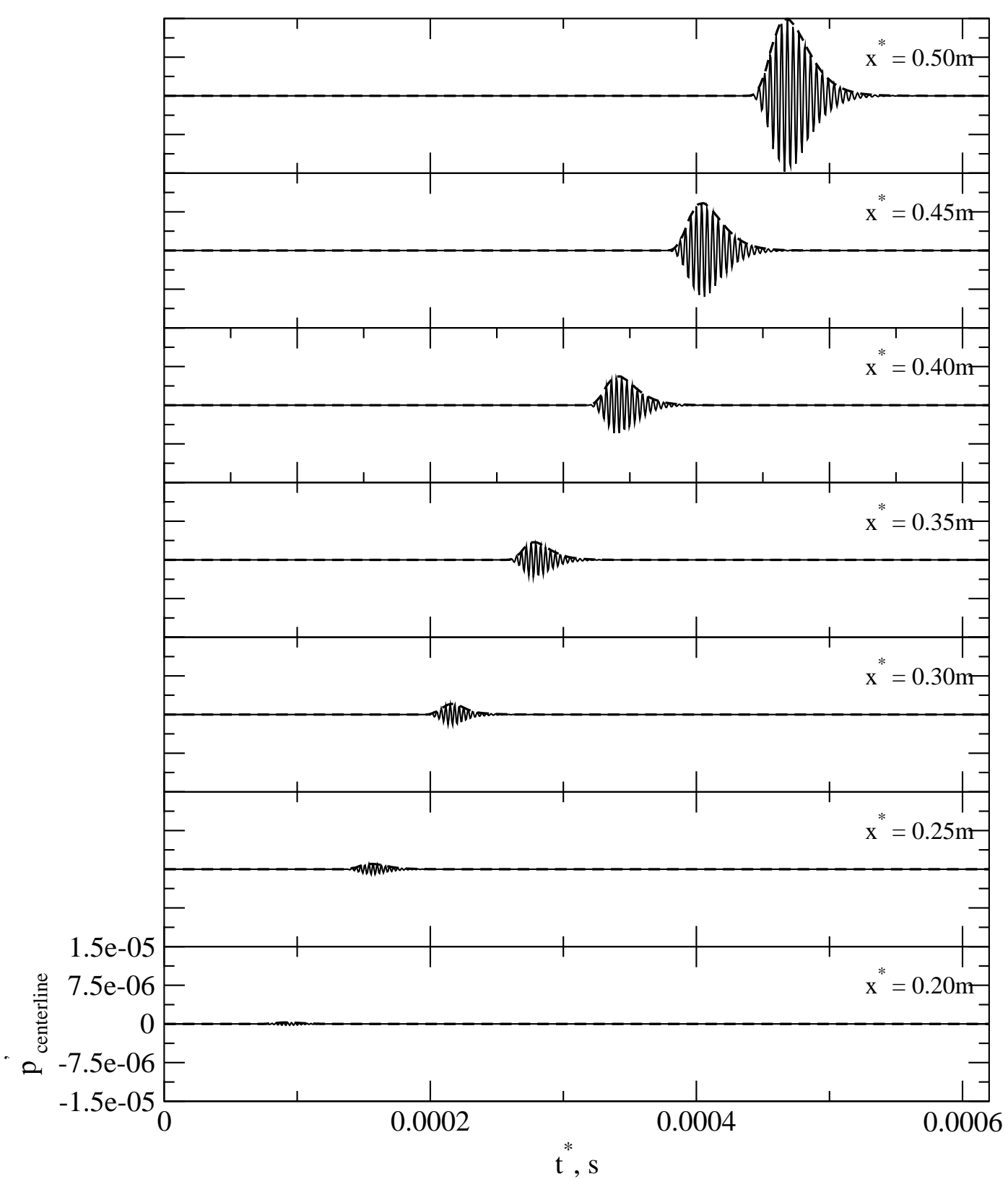

Figure 4.2: Temporal and spatial evolution of wall-pressure disturbance amplitude along the centerline for the linear wave packet. Solid lines represent the time signal and dashed lines its envelope. Isothermal Wall 


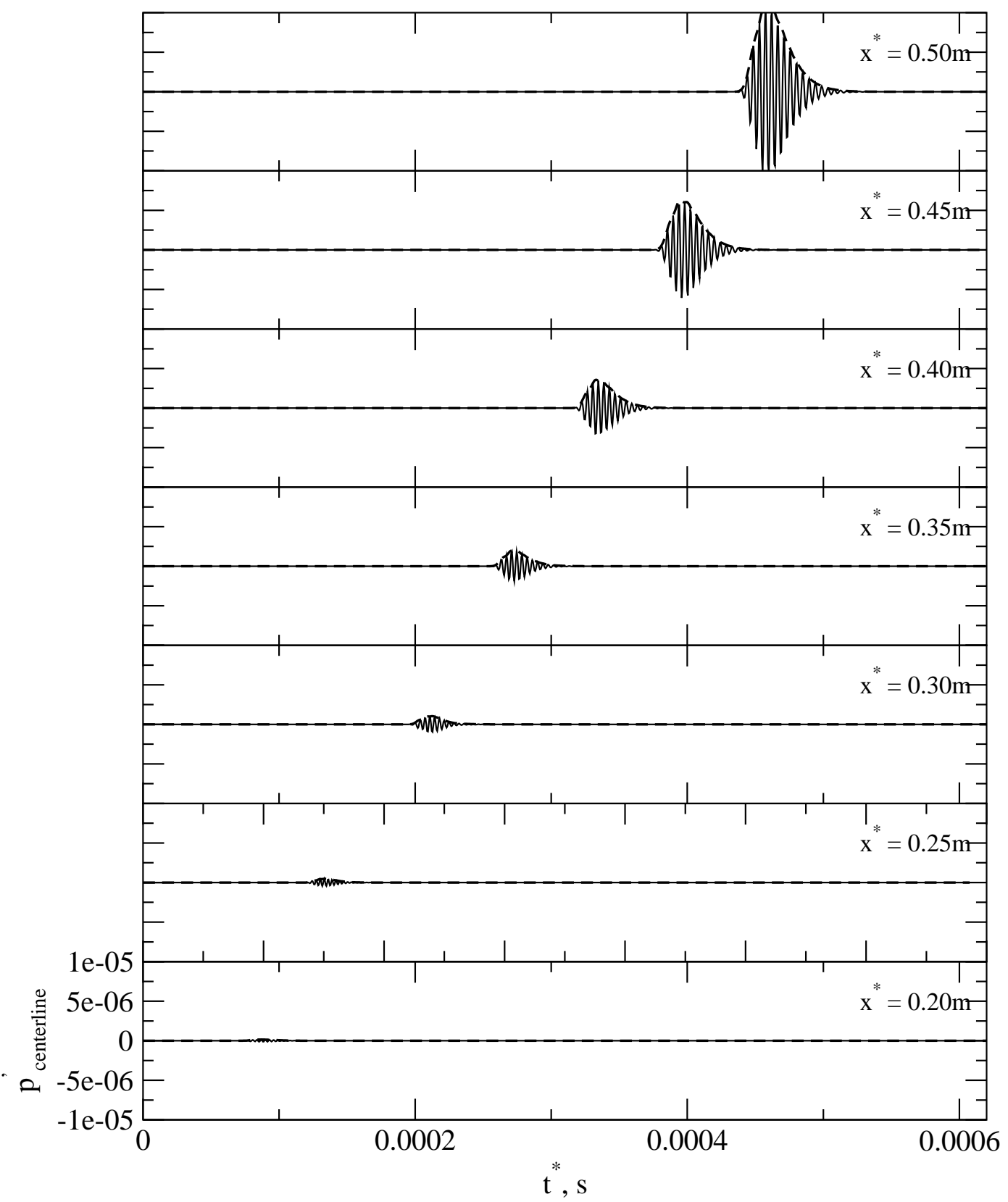

Figure 4.3: Temporal and spatial evolution of wall-pressure disturbance amplitude along the centerline for the linear wave packet. Solid lines represent the time signal and dashed lines its envelope. Adiabatic Wall 

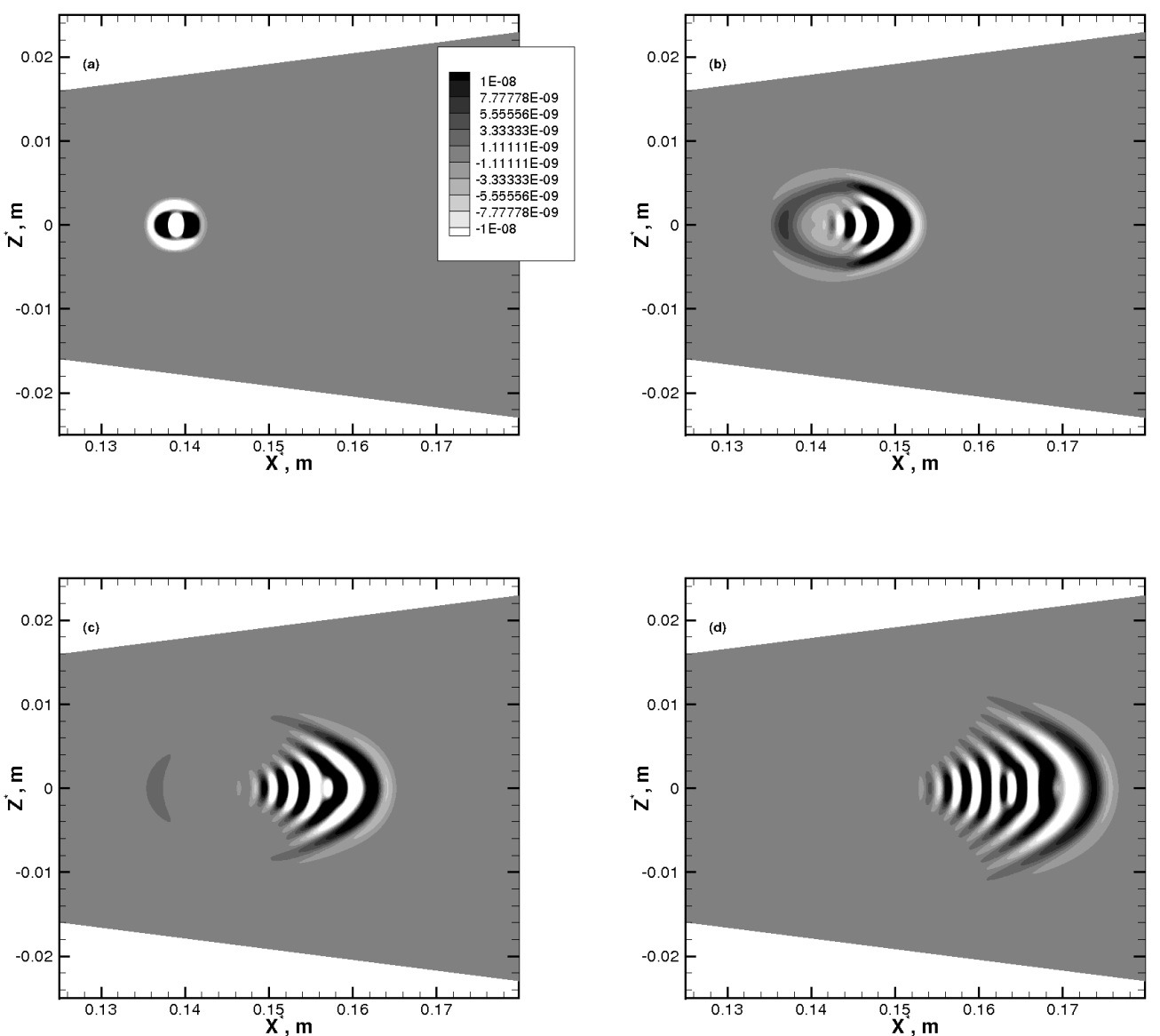

Figure 4.4: Snapshots of the linear wave packet showing the initial flow response to forcing through a hole on the cone surface. Shown are contours of wall-pressure disturbance on the unrolled cone surface at (a) $t^{*}=0.005 \mathrm{~ms}$ (b) $t^{*}=0.016 \mathrm{~ms}$ (c) $t^{*}=0.028 \mathrm{~ms}$ and $(\mathrm{d}) t^{*}=0.039 \mathrm{~ms}$. All the plots have the same contour levels. Isothermal Wall. 

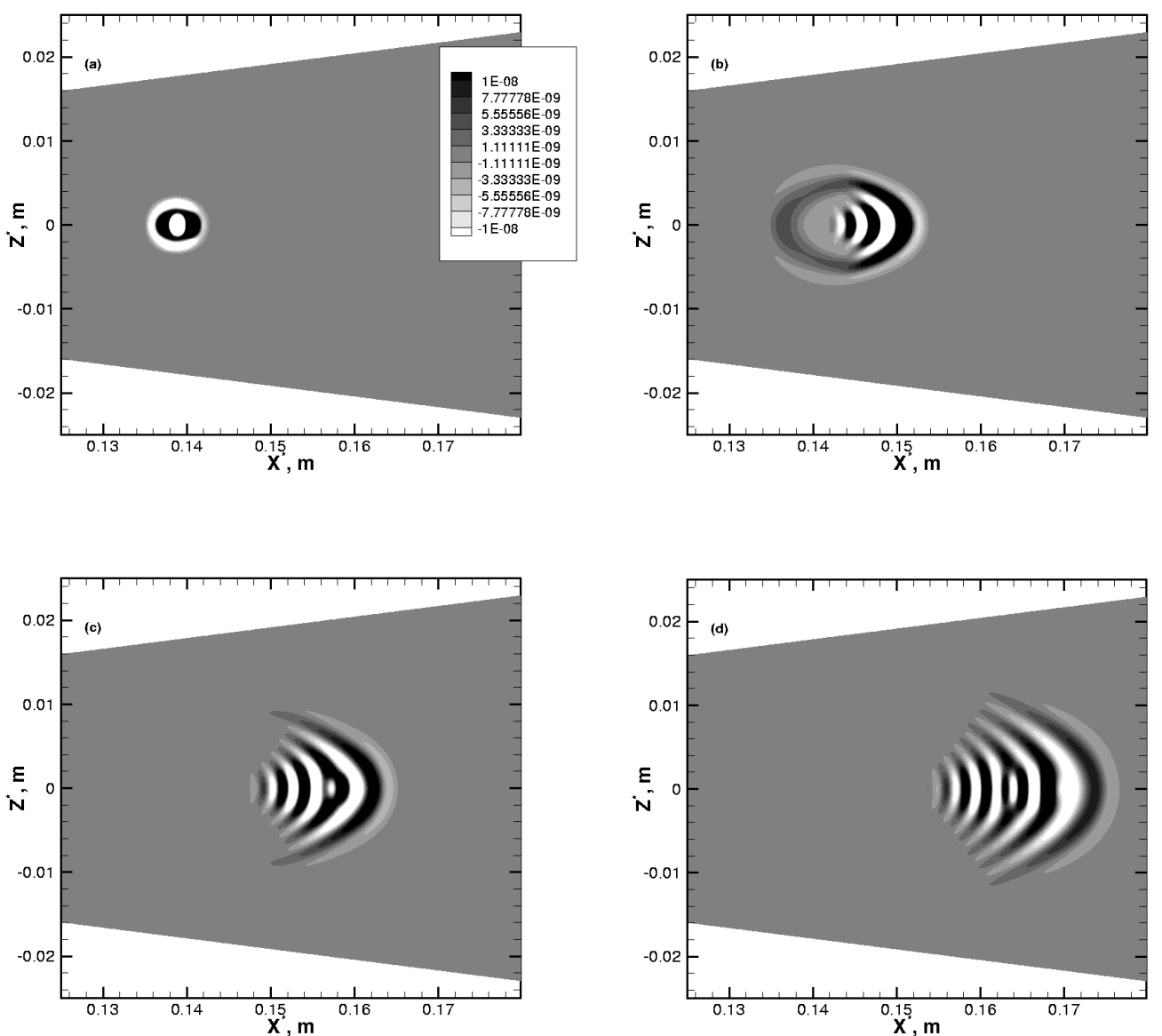

Figure 4.5: Snapshots of the linear wave packet showing the initial flow response to forcing through a hole on the cone surface. Shown are contours of wall-pressure disturbance on the unrolled cone surface at (a) $t^{*}=0.005 \mathrm{~ms}$ (b) $t^{*}=0.016 \mathrm{~ms}$ (c) $t^{*}=0.028 \mathrm{~ms}$ and $(\mathrm{d}) t^{*}=0.039 \mathrm{~ms}$. All the plots have the same contour levels. Adiabatic Wall. 
most likely first-mode waves, which have lower frequencies than the most amplified (dominant) axisymmetric waves. Like the axisymmetric waves, the oblique waves experience spatial and temporal growth as the wave packet propagates towards the outflow boundary of the computational domain as seen in figures $4.6 \mathrm{c}$ and $4.7 \mathrm{c}$. At $t^{*}=0.285 \mathrm{~ms}$ the acoustic waves that were seen propagating in front of the main wave packet have either almost (figure 4.6c) or completely (figure 4.7c) disappeared. Thus suggesting that these are the stable modes that continued to decay while propagating downstream. Also, it can be observed that the wave packet does not spread significantly in the azimuthal direction. Hence, the choice of the domain width of one-third of the cone circumference was large enough for the current investigation.

A close-up of the linear wave packets in the adiabatic and isothermal wall boundary layers is shown in figure 4.8 and 4.9. Two different contour levels are shown in order to illustrate that the flow structures seen in the wall pressure are strongly dependent on the chosen contour level. In figure 4.8a and 4.9a the contour levels were chosen such that the strongly amplified axisymmetric or two-dimensional waves and the weakly amplified low-frequency oblique first-mode waves are visible. In figure $4.8 \mathrm{~b}$ and $4.9 \mathrm{~b}$, however, the amplitude of the contour levels was increased to emphasize only the dominant axisymmetric waves.

\subsubsection{Disturbance Field}

For high-speed boundary layers (see, Balakumar \& Malik, 1992; Tumin, 2007; Fedorov, 2011), apart from the discrete modes seven branches of the continuous spectrum in the complex wavenumber space exist, four of which (two acoustic waves, one vorticity wave and one entropy wave) contribute to the flow field downstream of the disturbance source. The discrete spectrum of disturbances in high-speed boundary layers is clearly more complicated than in the low-speed case. At high Mach numbers there are two discrete modes (stable and unstable). Balakumar \& Malik (1992) 

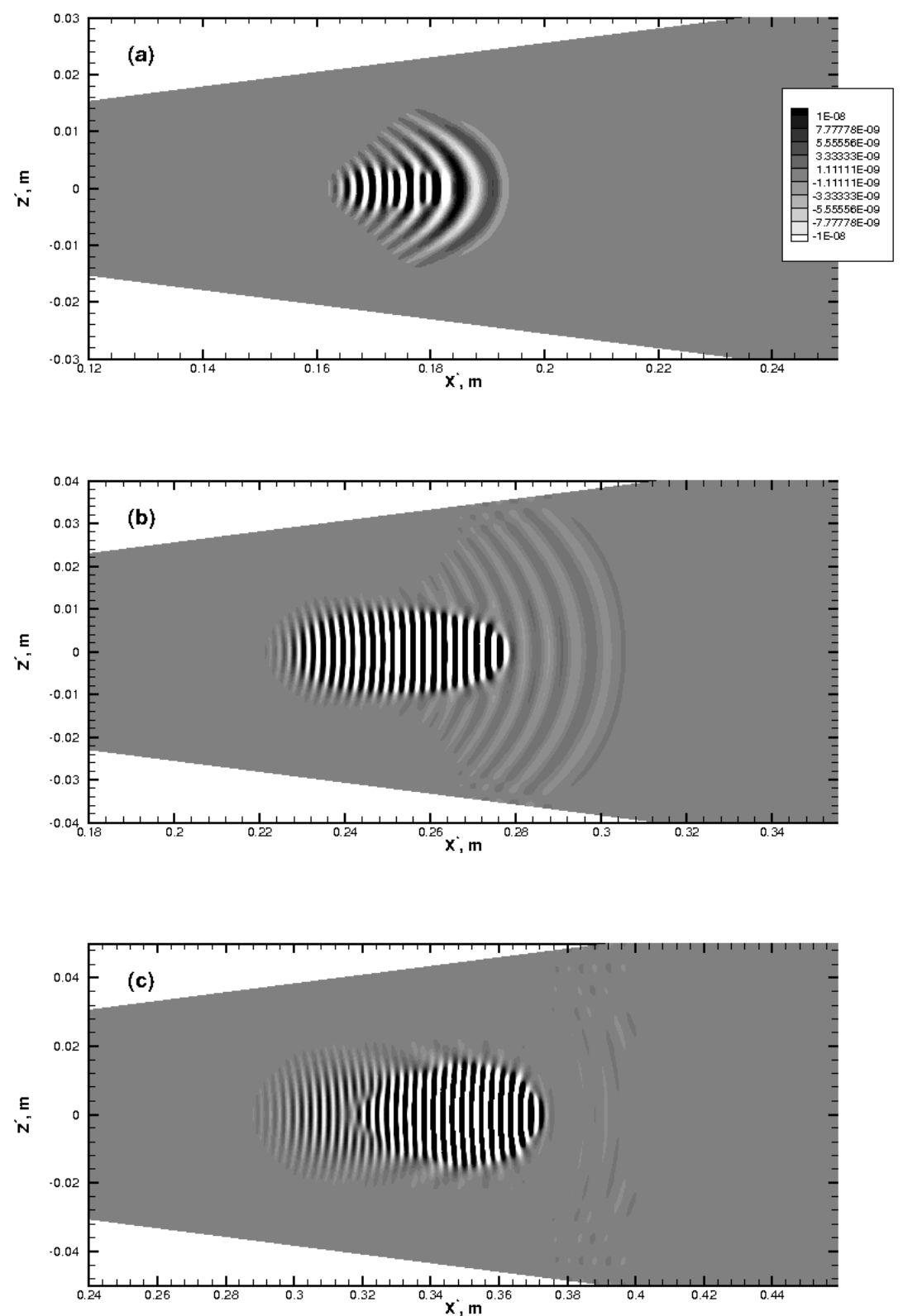

Figure 4.6: Snapshots of the linear wave packet illustrating its development in downstream direction. Shown are contours of wall-pressure disturbance on the unrolled cone surface at (a) $t^{*}=0.056 \mathrm{~ms}$ (b) $t^{*}=0.171 \mathrm{~ms}$ and (c) $t^{*}=0.285 \mathrm{~ms}$. All the plots have the same contour levels. Isothermal Wall. 

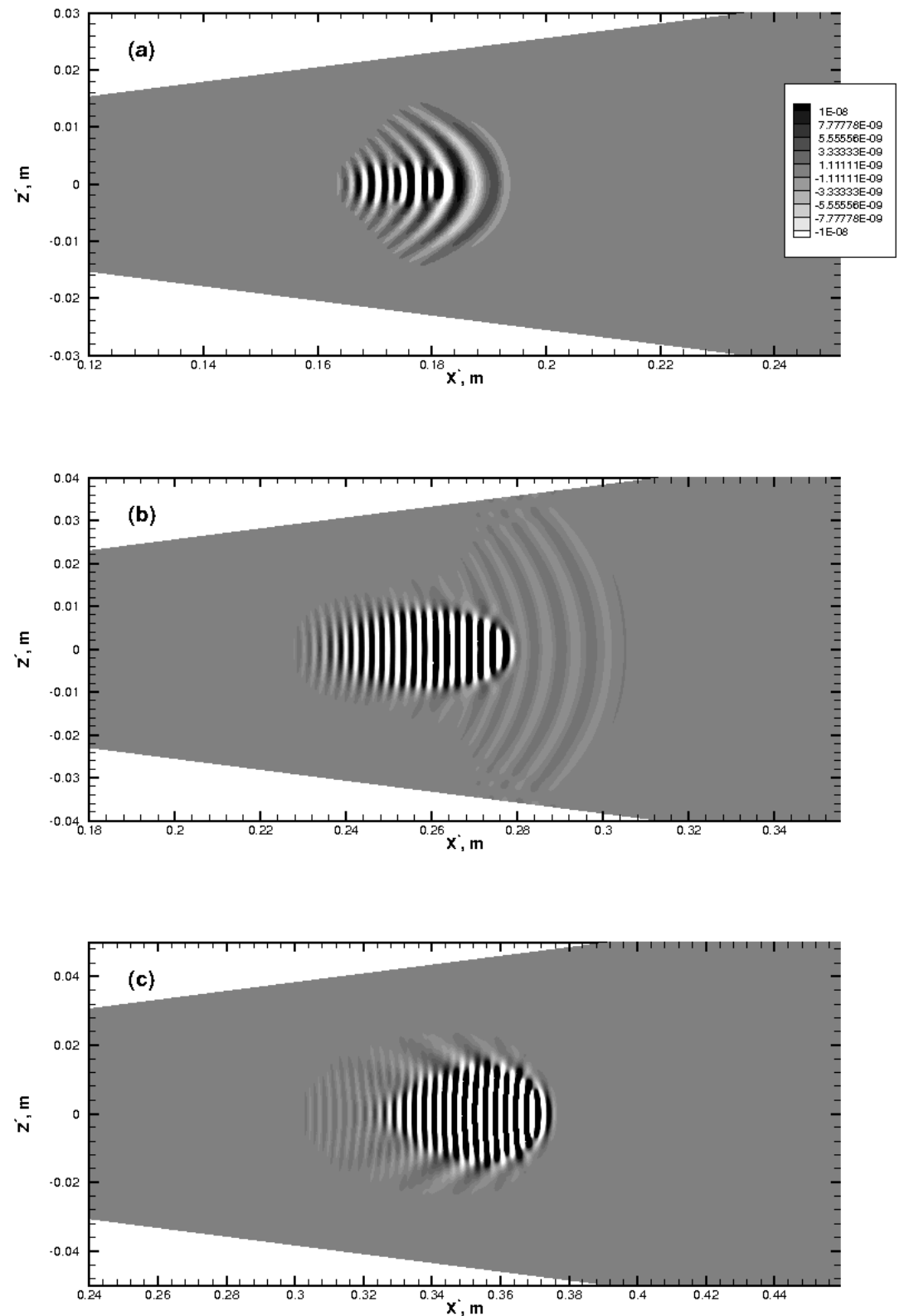

Figure 4.7: Snapshots of the linear wave packet illustrating its development in downstream direction. Shown are contours of wall-pressure disturbance on the unrolled cone surface at (a) $t^{*}=0.056 \mathrm{~ms}$ (b) $t^{*}=0.171 \mathrm{~ms}$ and (c) $t^{*}=0.285 \mathrm{~ms}$. All the plots have the same contour levels. Adiabatic Wall. 

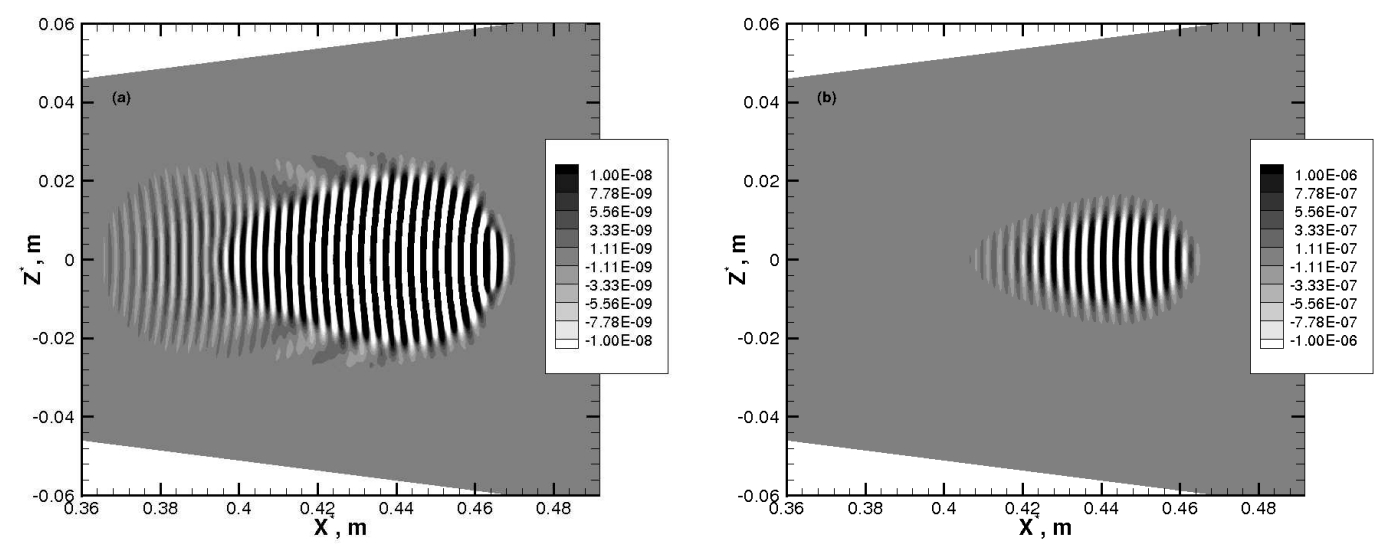

Figure 4.8: Close-up view of the linear wave packet at time $t^{*}=0.4 \mathrm{~ms}$. Shown are contours of wall-pressure disturbance using different contour levels for visualization. Isothermal Wall.
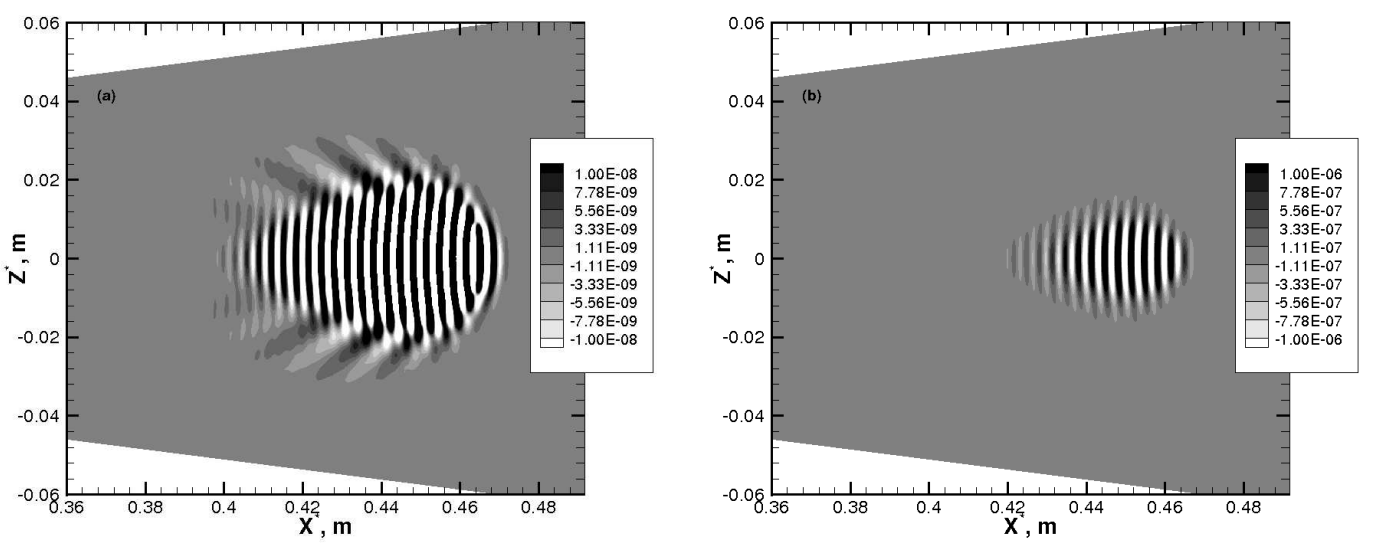

Figure 4.9: Close-up view of the linear wave packet at time $t^{*}=0.4 \mathrm{~ms}$. Shown are contours of wall-pressure disturbance using different contour levels for visualization. Adiabatic Wall. 
showed that receptivity coeffcients for the stable discrete modes are much larger than those for the unstable mode. Hence, the flow near the disturbance source is dominated by the continuous spectrum and the stable discrete modes. Further away from the disturbance source the unstable discrete mode dominates. Therefore, both the stable and unstable discrete modes are of interest for understanding transition mechanisms in hypersonic boundary layers.

By tracking the eigenvalues of the unstable slow discrete mode as obtained from LST, the stability diagrams as shown in figures 4.10 and 4.11 for the isothermal and adiabatic wall cases can be constructed. Contours of constant amplification rate, $\alpha_{i}$, are shown in the $R_{x}-F$ plane for $k_{c}=0$ (axisymmetric disturbance waves). LST results, are generated using the linear stability solver from Mack (1965, 1969, 1987). Note that Mack's linear stability solver is based on the flat-plate equations and do not account for transverse curvature and body divergence. However, it accounts for the change in azimuthal wavelength with the streamwise location. For the linear stability analysis using the Mack solver, self-similar compressible boundary layer profiles were used. The first- and second-mode unstable regions are clearly visible in figure 4.10 and 4.11. For the isothermal wall case the first-mode and second-mode unstable frequency bands are separated by a stable region. However, for the adiabatic wall case, the two frequency bands appear connected. The contour levels for the growth rate of second-mode waves have a maximum value of -0.0073 and -0.0065 for the isothermal and adiabatic wall cases respectively. The contour values indicates that cooling the surface promotes the second-mode instabilty and accelerates the growth of that mode. Furthermore, figure 4.12 compares the growth rates calculated with isothermal and adiabatic wall temperatures for several streamwise locations (indicated by local Reynolds numbers). At all locations the effect of wall cooling (isothermal or cold wall case) becomes apparent; as mentioned before it stabilizes the first-mode disturbances and destabilizes the second-mode disturbances. As the isothermal wall condition produces a thinner boundary layer, the second-mode frequency bands have 


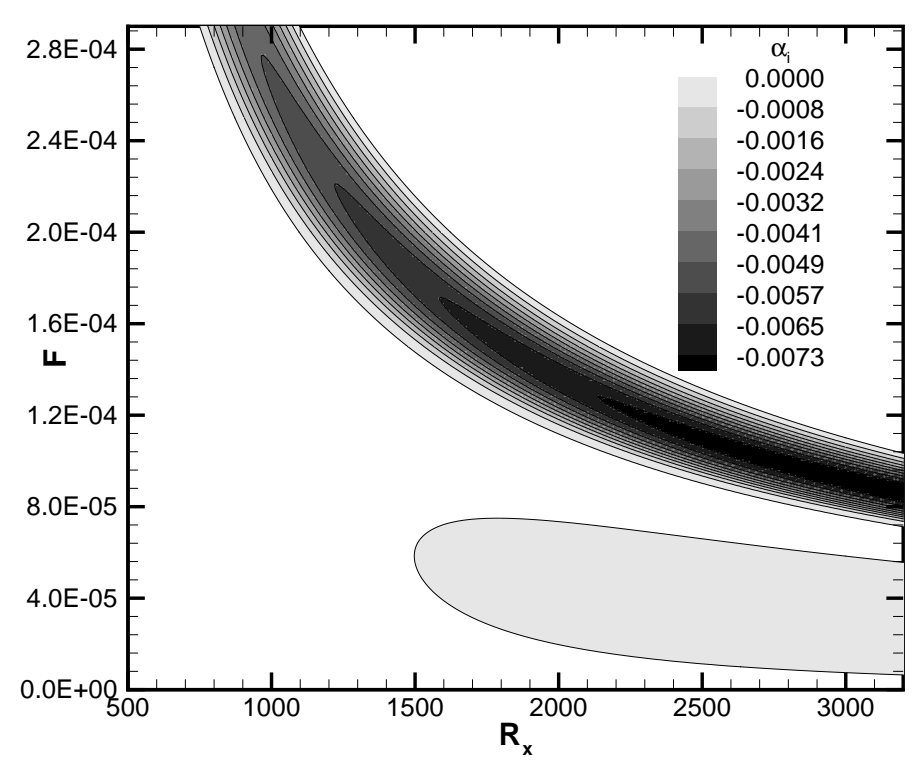

Figure 4.10: Contours of constant amplification rate $\alpha_{i}$ for axisymmetric disturbances $\left(k_{c}=0\right)$. Generated using Mack's LST solver (Mack, 1965, 1987). Isothermal Wall.

shifted to a slightly higher frequency. This shift in the frequency bands could be observed for all four streamwise locations plotted in figure 4.12.

Any linear disturbance can be considered to be composed of a sum of independent normal modes, each of which behaves exactly as if it were isolated and excited by a separate periodic disturbance source. Therefore, the linear wave packet here can be treated like this and by tracking all wave components of the wave packet from the location of the hole to the outflow, the amplitudes, growth rates and wave angles of all individual wave components were determined. In particular, disturbance amplitudes, wall-normal amplitude profiles "eigenfunctions" and phase were obtained by performing Fourier transformations in time. In numerical simulations using the complete Navier-Stokes equations, therefore including non-parallel effects, the spatial growth rate, $\alpha_{i}$ depends on the criterion used. The specific criterion or quantity used to calculate the growth rate will affect not only the absolute value of the growth rate, but also the position of the neutral curve. Fasel \& Konzelmann (1990) demon- 


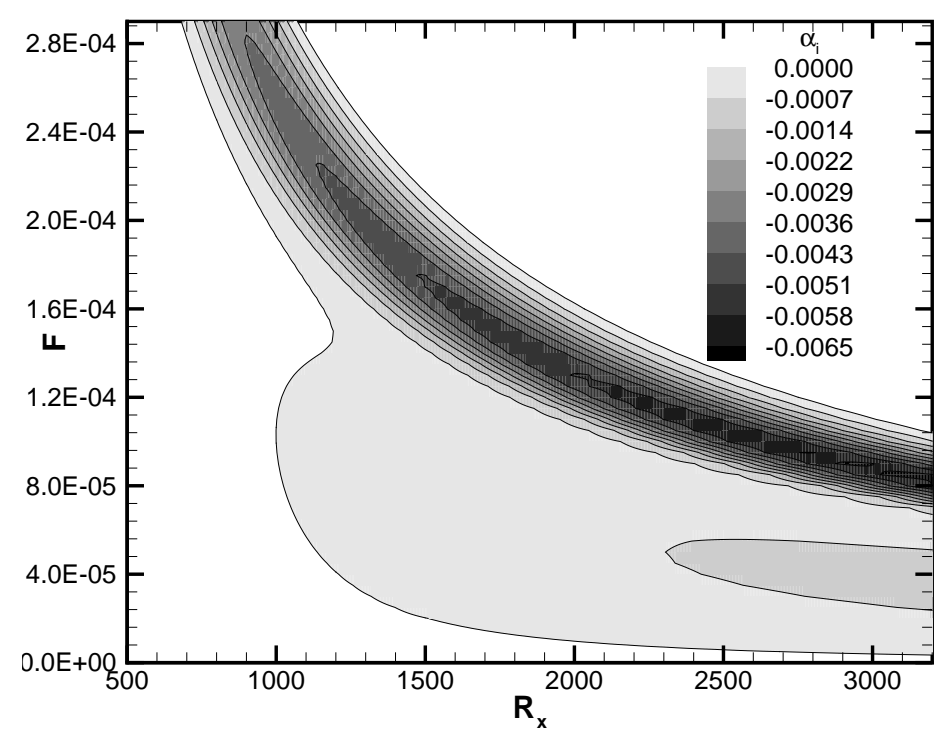

Figure 4.11: Contours of constant amplification rate $\alpha_{i}$ for axisymmetric disturbances $\left(k_{c}=0\right)$. Generated using Mack's LST solver (Mack, 1965, 1987). Adiabatic Wall.

strated this issue for an incompressible flat-plate boundary layer by applying various criteria to calculate the spatial growth rate of Tollmien-Schlichting waves. A comparison of streamwise wavelength, $\alpha_{r}$ and spatial growth rates, $\alpha_{i}$ for four axisymmetric disturbance waves with different frequencies $(F=1.04 E-04, F=1.09 E-04$, $F=1.15 E-04, F=1.23 E-04)$ is shown in figure 4.13 and 4.14 . Here, the spatial growth rate and the streamwise wavenumber are calculated based on the wall pressure disturbance as follows:

$$
\alpha_{i}=-\frac{d}{d x}\left[\ln \left(\left.A(x)\right|_{p_{w a l l}^{\prime}}\right)\right], \quad \alpha_{r}=\frac{d}{d x}\left[\left.\theta(x)\right|_{p_{\text {wall }}^{\prime}}\right] .
$$

In general, the streamwise wave number $\alpha_{r}$ is less sensitive to the criterion used and therefore less sensitive to the non-parallel effects. Hence the agreement between streamwise wave number from DNS and LST is very good for all the four frequencies shown in figures 4.13 and 4.14. Close to the forcing location the streamwise wave 

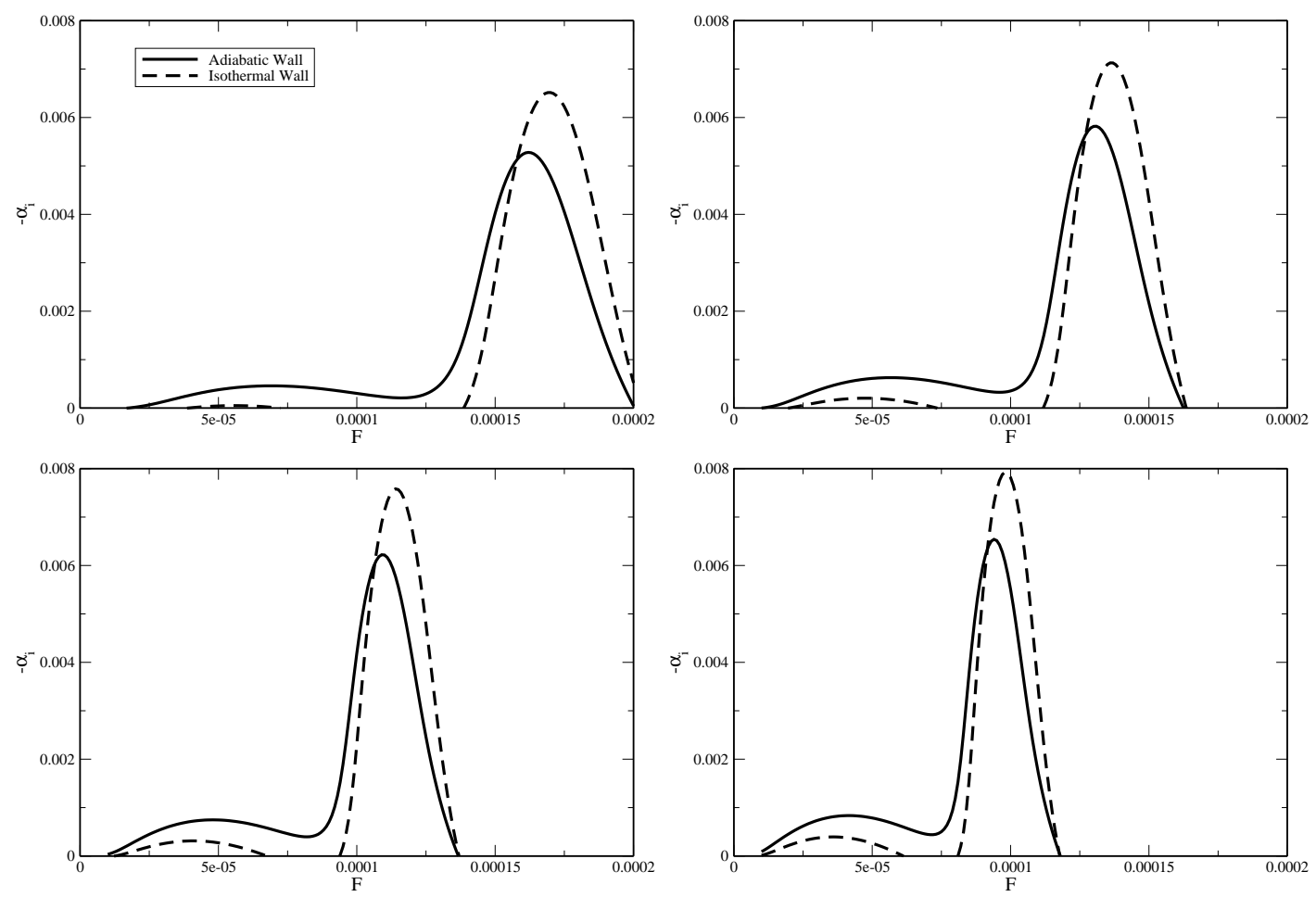

Figure 4.12: Comparison of spatial growth rates of axisymmetric disturbance waves $\left(k_{c}=0\right)$ obtained for adiabatic and isothermal wall cases at (a) $R_{x}=1600$ (top left) (b). $R_{x}=2000$ (top right) (c) $R_{x}=2400$ (bottom left) (d) $R_{x}=2800$ (bottom right). Growth rates are obtained using Mack's LST solver (Mack, 1965, 1987) and are plotted as a function of reduced frequency $F$. 
number $\alpha_{r}$ and the spatial growth rate $\alpha_{i}$ calculated from DNS is modulated by the superposition of damped waves. This modulation is more pronounced for the spatial amplification rate $\alpha_{i}$ than for the streamwise wave number $\alpha_{r}$. Close to the location where waves exhibit maximum spatial amplification there is a better agreement between the spatial amplification rate $\alpha_{i}$ calculated from DNS and LST. The reason for the small differences in spatial growth rate $\alpha_{i}$ at other streamwise locations is likely due to non-parallel effects.

The wall-normal amplitude and phase distributions for the streamwise velocity disturbance and pressure disturbance from DNS are compared to LST results in figure 4.15. The amplitude and phase distributions are taken at the downstream location $x^{*}=0.472 m\left(R_{x}=2600\right)$ for an axisymmetric disturbance wave with frequency $F=1.04 E-04$. The amplitude distributions from DNS and LST are normalized by their respective maximum values. There is a good agreement between DNS and LST results in figure 4.15, indicating that the simulations capture the linear disturbance development accurately.

The disturbance spectra obtained from Fourier transformation of the wall-pressure disturbance using 500 Fourier modes in time are shown in figures 4.16, 4.17 and 4.18. The broad disturbance spectrum produced by the 3D pulse is shown in figure 4.16. A strong peak can be seen in the spectrum, which corresponds to the pulse excitation. A closer look will reveal that the peak appears for a slightly higher frequency on the spectrum for the isothermal wall case. This may be due to the different receptivity of the thinner isothermal wall boundary layer. The streamwise development of the disturbance spectrum is shown in figures 4.17 and 4.18 for the two cases. As expected, the spectra exhibit a maximum at the azimuthal mode number $k_{c}=0$ for both cases at all streamwise locations, because according to linear stability theory, axisymmetric disturbances have higher amplification rates than corresponding $3 D$ oblique waves. In both cases, at subsequent downstream locations $\left(x^{*}=0.250 m, x^{*}=0.350 m, x^{*}=\right.$ $0.450 \mathrm{~m}$, and $\left.x^{*}=0.550 \mathrm{~m}\right)$, the spectrum broadens and spreads over higher azimuthal 

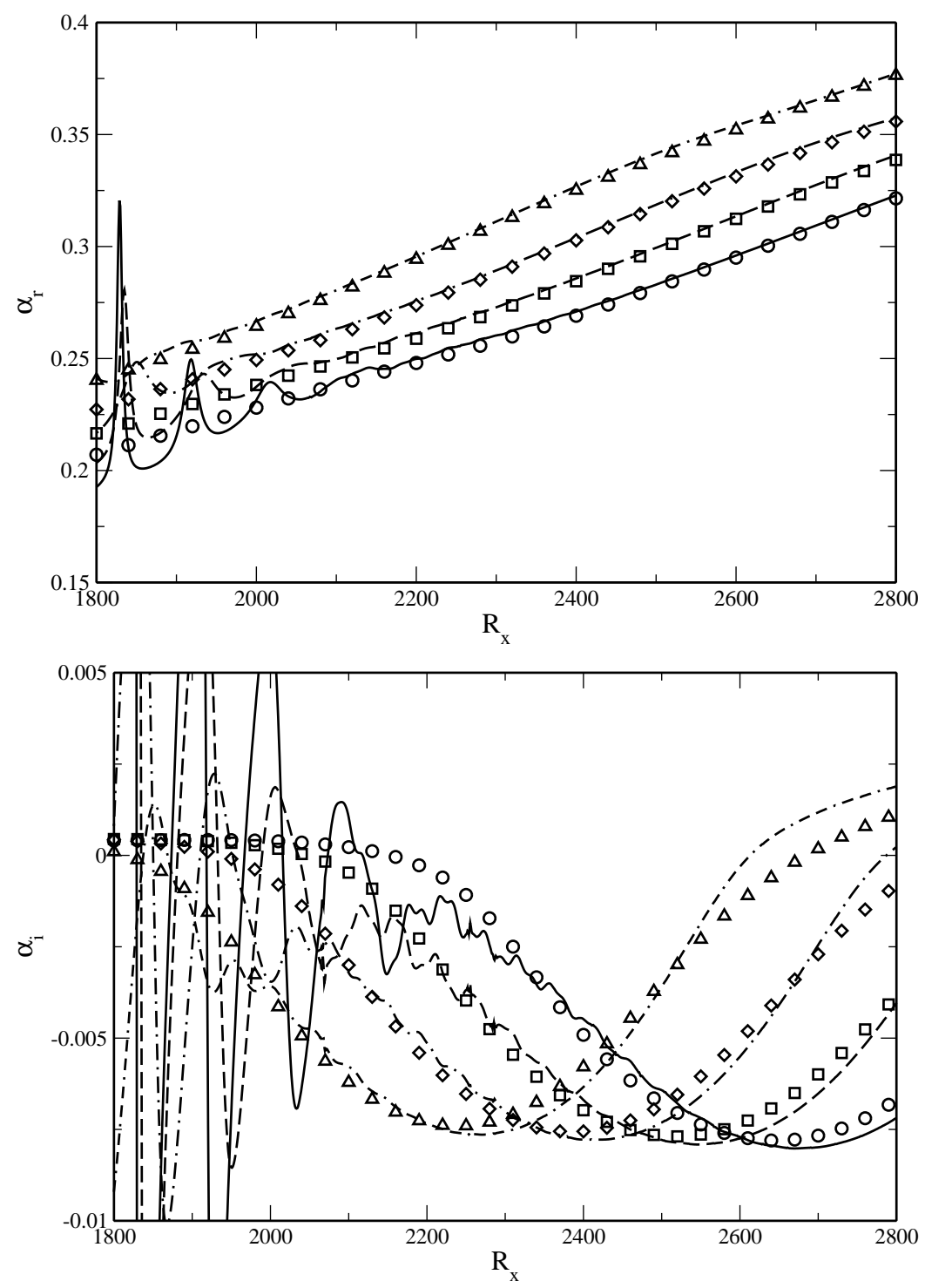

Figure 4.13: Downstream development of the complex streamwise wavenumber (streamwise wave number (top) and spatial amplification rate (bottom)) predicted by DNS (from wall pressure disturbance) and LST. Streamwise wave number $\left(\alpha_{r}\right)$ and spatial growth rates $\left(\alpha_{i}\right)$ are compared for four axisymmetric disturbance waves $\left(k_{c}=0\right)$ with different frequencies. Lines represent predictions by DNS and symbols represent predictions by LST. The frequencies are (a) $F=1.04 E-04(-$, o) (b) $F=1.09 E-04(---, \square)(\mathrm{c}) F=1.15 E-04(-.-.-, \diamond)$ and $(\mathrm{d}) F=1.23 E-04$ $(--.--, \triangle)$. LST results are computed using Mack's solver (Mack, 1965, 1987). Isothermal Wall. 

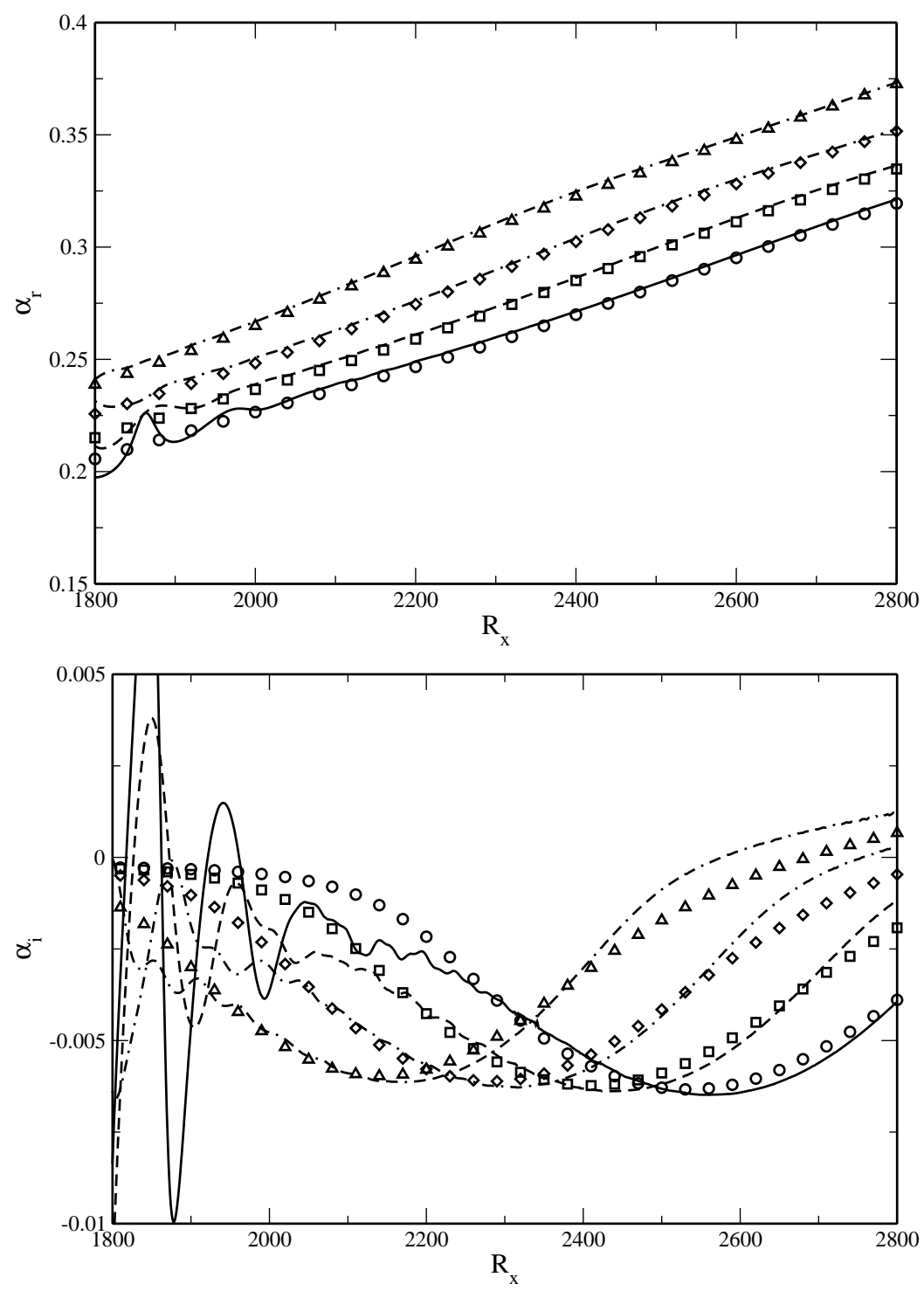

Figure 4.14: Downstream development of the complex streamwise wavenumber (streamwise wave number (top) and spatial amplification rate (bottom)) predicted by DNS (from wall pressure disturbance) and LST. Streamwise wave number $\left(\alpha_{r}\right)$ and spatial growth rates $\left(\alpha_{i}\right)$ are compared for four axisymmetric disturbance waves $\left(k_{c}=0\right)$ with different frequencies. Lines represent predictions by DNS and symbols represent predictions by LST. The frequencies are (a) $F=1.04 E-04(-, \circ)$ (b) $F=1.09 E-04(---, \square)(\mathrm{c}) F=1.15 E-04(-.-.-, \diamond)$ and $(\mathrm{d}) F=1.23 E-04$ $(--.--, \triangle)$. LST results are computed using Mack's solver (Mack, 1965, 1987). Adiabatic Wall. 

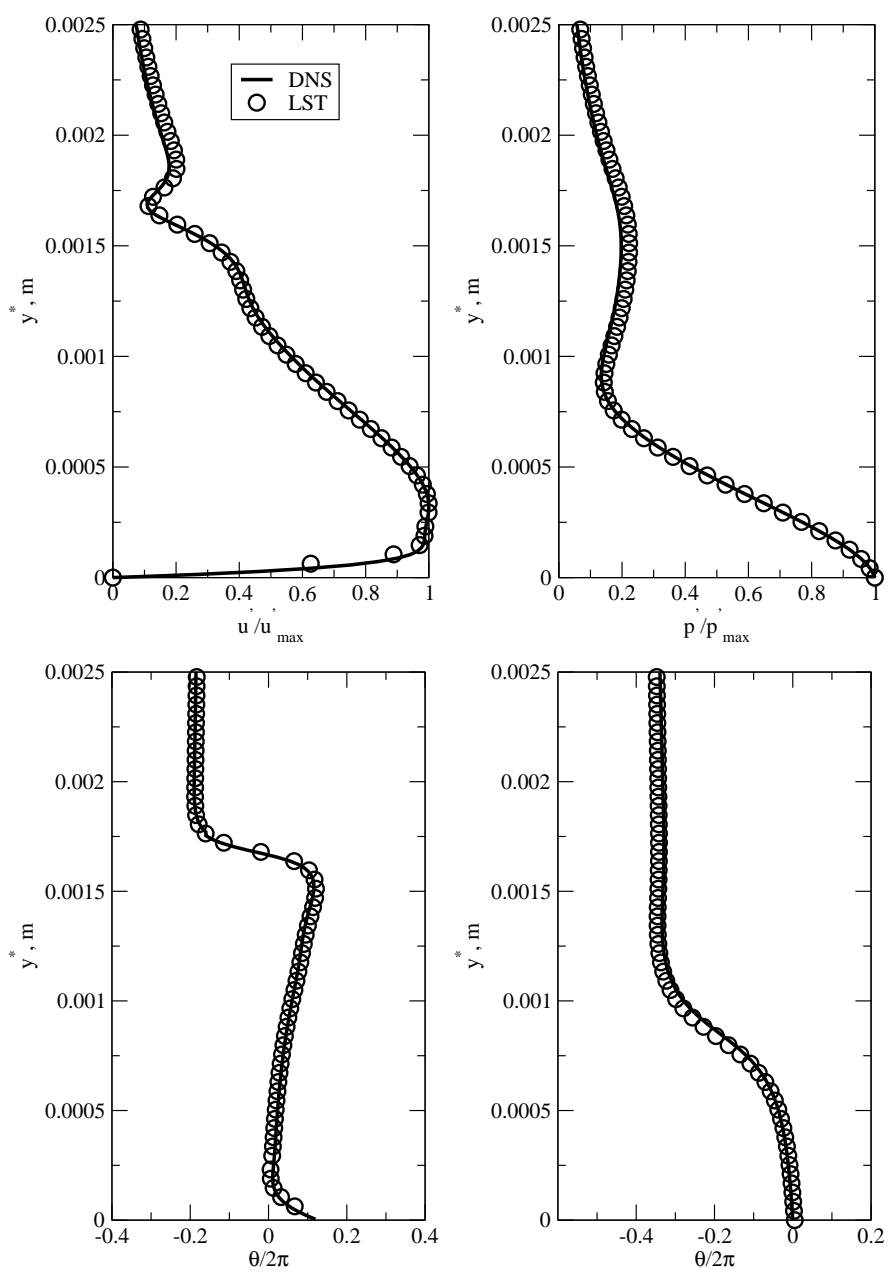

Figure 4.15: Comparison of wall-normal amplitude (top two plots) and phase distribution (bottom two plots) of the streamwise velocity and pressure disturbance to theoretical predictions from LST for frequency $F=1.04 E-04$ and azimuthal mode number $k_{c}=0$ (axisymmetric disturbance wave) at $R_{x}=2600$. LST results are computed using Mack's solverMack $(1965,1987)$. Adiabatic wall. 

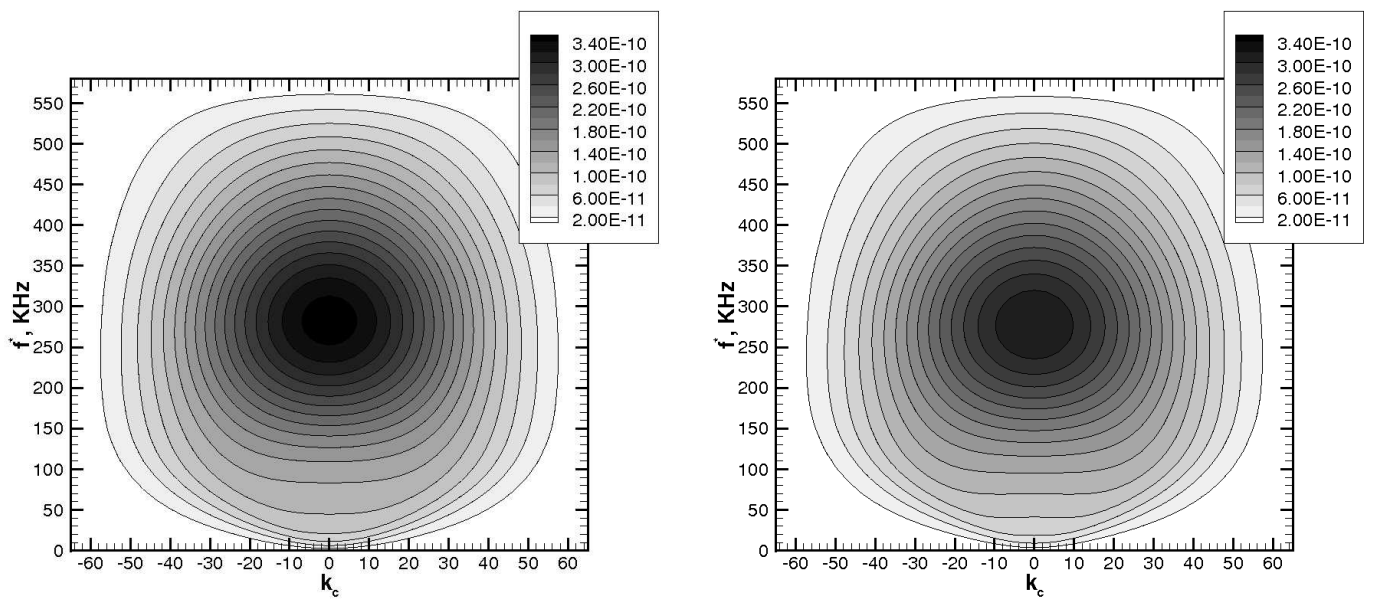

Figure 4.16: Disturbance spectrum in the azimuthal mode number - frequency $\left(k_{c}-\right.$ $\left.f^{*}\right)$ plane at the forcing location $\left(x^{*}=0.138 m\right)$ for the simulation with low forcing amplitude. Obtained from isothermal (left) and adiabatic (right) wall cases.

mode numbers. At the same time, lower frequency bands also becomes visible, making it appear as if the higher amplitude second-mode frequency band develops "legs." Similar to the higher second-mode-frequency band, the lower-frequency band is also amplified in the streamwise direction. However, unlike the second mode frequency band, the "legs" develop for oblique waves centered at around $k_{c} \pm 30$ for the isothermal wall case (figure4.17d) and at around $k_{c} \pm 25$ for the adiabatic wall case (figure4.18d). These low-frequency bands represent the first-mode oblique disturbances that are only weakly amplified when compared to the high-frequency second-mode waves and it appears slightly stronger in the adiabatic wall case than in the isothermal (cold) wall case, again confirming the stabilizing effect of wall cooling on the first-mode disturbances. Overall, the peak amplitude remains fixed at $k_{c}=0$ for both cases but shifts to lower frequencies as the boundary layer grows and becomes thicker in the downstream direction. 

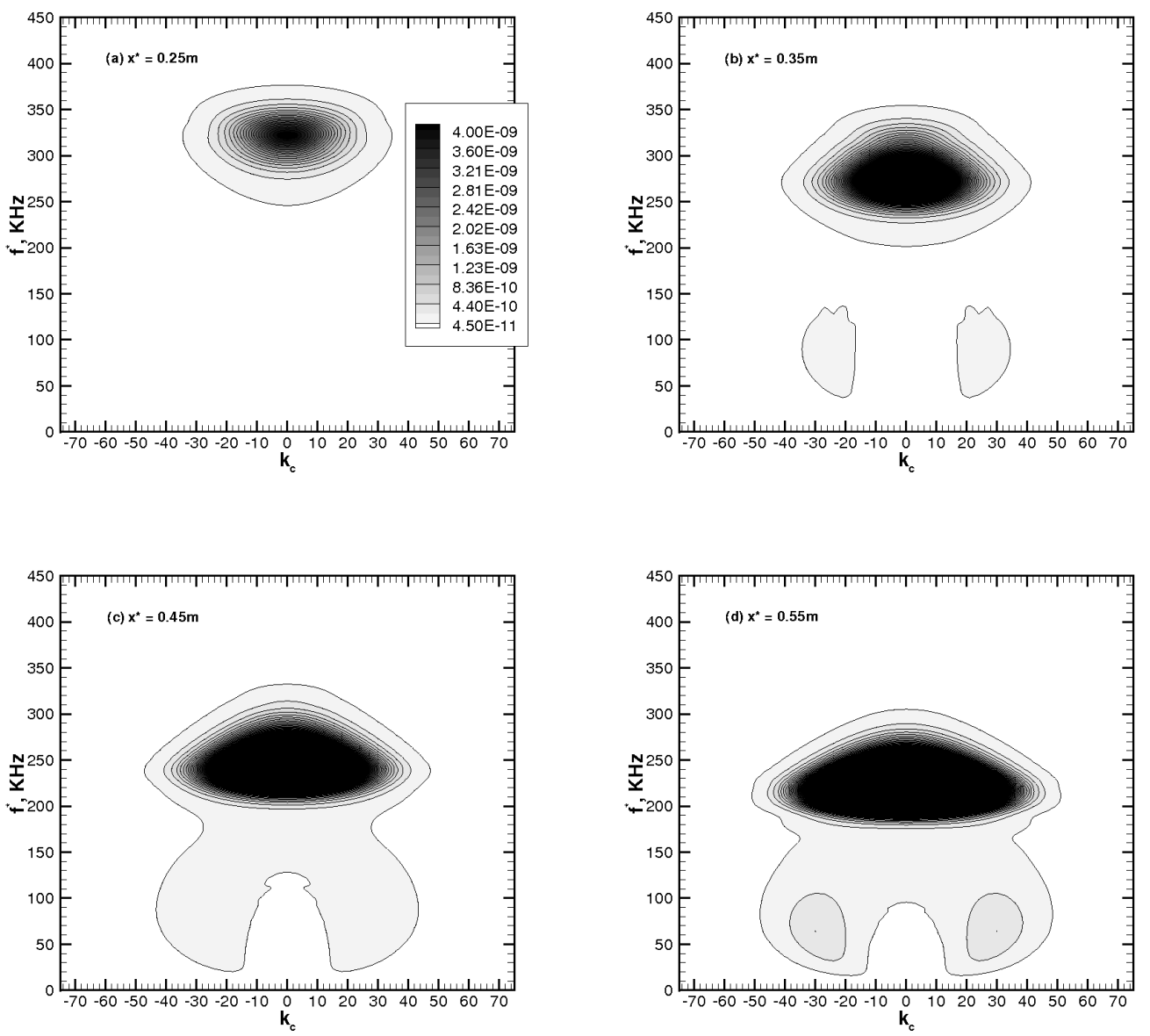

Figure 4.17: Disturbance spectrum in the azimuthal mode number - frequency $\left(k_{c}-\right.$ $\left.f^{*}\right)$ plane for the simulation with low forcing amplitude obtained from wall-pressure at different streamwise positions. (a) $x^{*}=0.250 \mathrm{~m}$, (b) $x^{*}=0.350 \mathrm{~m}$, (c) $x^{*}=0.450 \mathrm{~m}$, and $(\mathrm{d}) x^{*}=0.550 \mathrm{~m}$. All the plots have the same contour levels. Isothermal wall. 

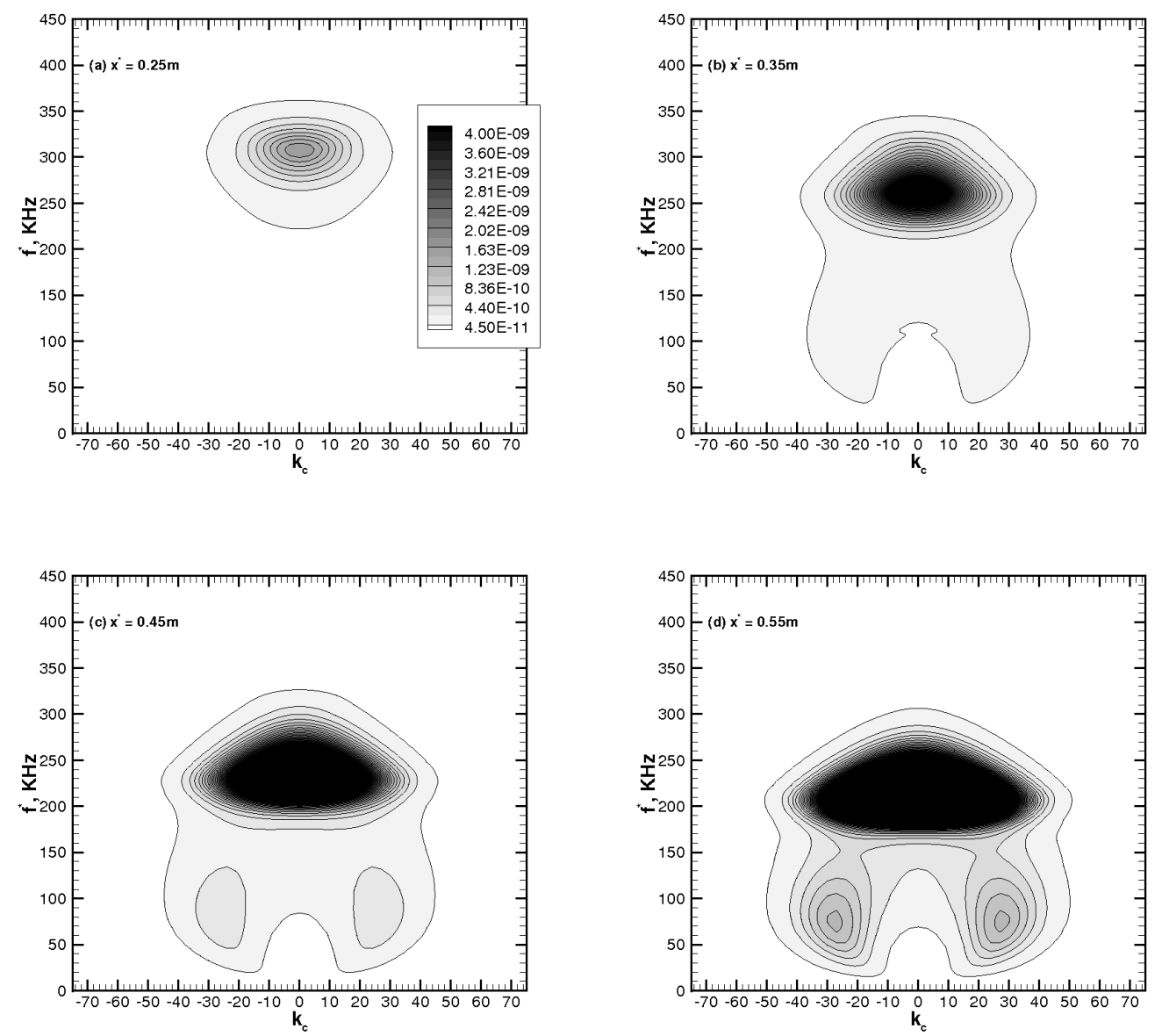

Figure 4.18: Disturbance spectrum in the azimuthal mode number - frequency $\left(k_{c}-\right.$ $\left.f^{*}\right)$ plane for the simulation with low forcing amplitude obtained from wall-pressure at different streamwise positions. (a) $x^{*}=0.250 \mathrm{~m}$, (b) $x^{*}=0.350 \mathrm{~m}$, (c) $x^{*}=0.450 \mathrm{~m}$, and (d) $x^{*}=0.550 \mathrm{~m}$. All the plots have the same contour levels. Adiabatic wall. 


\subsubsection{Comparison with flat plate}

The linear transition regime of a hypersonic flat-plate boundary layer was investigated by introducing a similar short-duration (localized) three-dimensional pulse disturbance with a very small amplitude (0.001\% of the free-stream velocity). The flow parameters used in the flat-plate simulation matched the edge values of the sharp cone boundary layer discussed above, thereby, facilitating a direct comparison between the development of a localized pulse disturbance in a sharp cone and a flatplate boundary layer. Only an isothermal wall boundary condition $\left(T_{w}=300 \mathrm{~K}\right)$ was considered for the flat-plate simulation. As in the case of a cone, the pulse disturbance develops into a three-dimensional wave packet, which propagates downstream. The temporal and spatial evolution of the centerline wall-pressure disturbance amplitude is shown in figure 4.19. Envelopes of these time signals (Gaster \& Grant, 1975) are also included in figure 4.19. In this figure, a scale given with the bottom signal indicates the disturbance amplitude. As for the cone, the wave packet spreads and its amplitude increases as it propagates downstream. However, it could be observed that the waves within the wave packet on a flat-plate has a larger streamwise wave length than in the case of cone (see, figure 4.2).

Amplitudes, wall-normal amplitude profiles "eigenfunctions" and phases were obtained from Fourier transformations of the data in time. Results obtained from the linear flat-plate wave packet simulation were compared to results from Linear Stability Theory (LST). Similar to the cone investigations, for comparison with LST, the linear stability solver from Mack $(1965,1969,1987)$ was employed. Again as before, for LST analysis using the Mack solver, self-similar compressible flat-plate boundary layer profiles were used for the baseflow.

In figure 4.20, contours of constant amplification rate, $\alpha_{i}$, are shown in the $R_{x}-F$ plane for spanwise mode number, $k=0$ (2D disturbance waves). The stability diagram in figure 4.20 looks very similar to the diagram obtained for the cone (fig- 


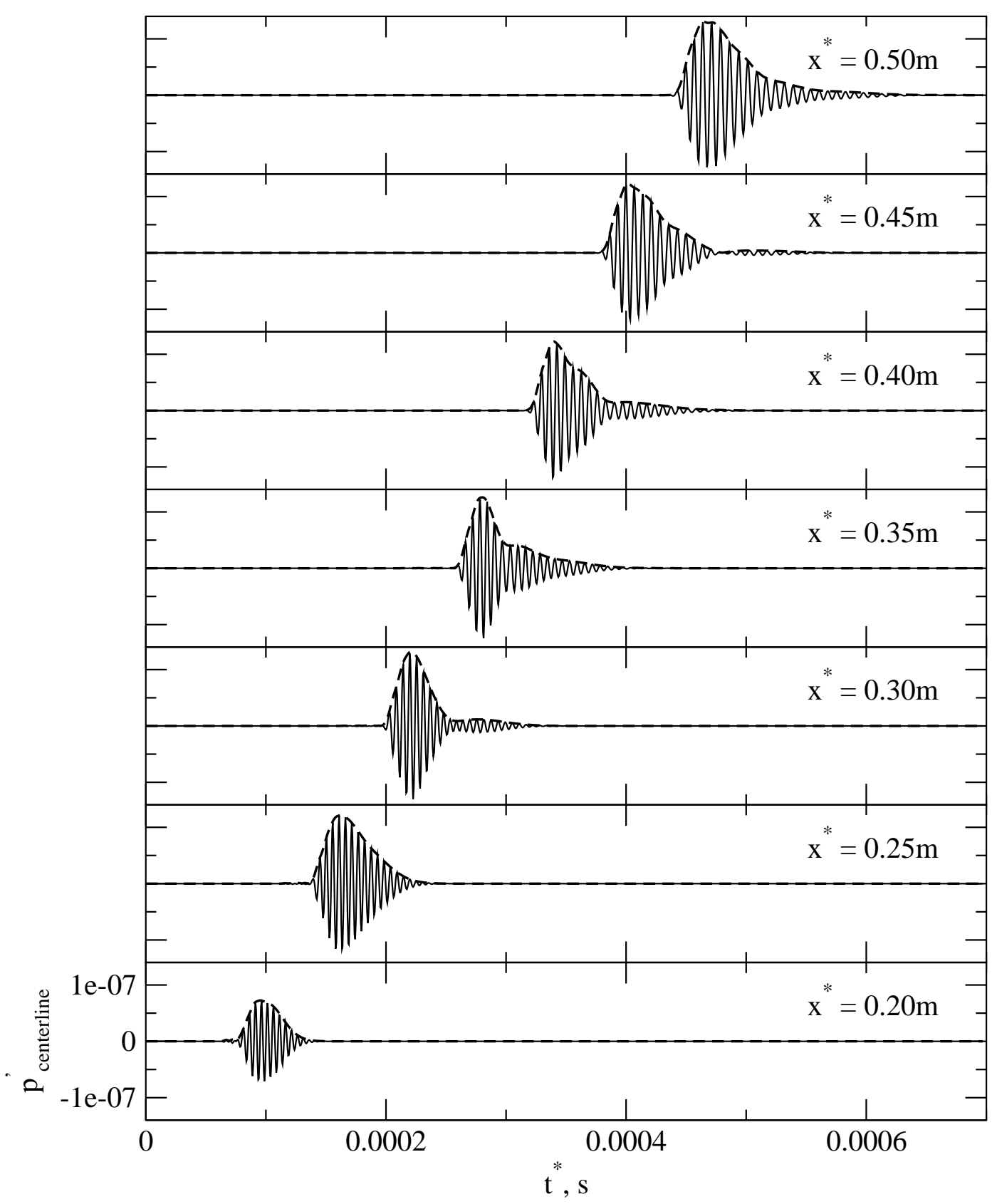

Figure 4.19: Temporal and spatial evolution of wall-pressure disturbance amplitude along the centerline for the linear wave packet on a flat-plate. Solid lines $(-)$ represent the time signal and dashed lines (- - ) its envelope. Isothermal wall. 


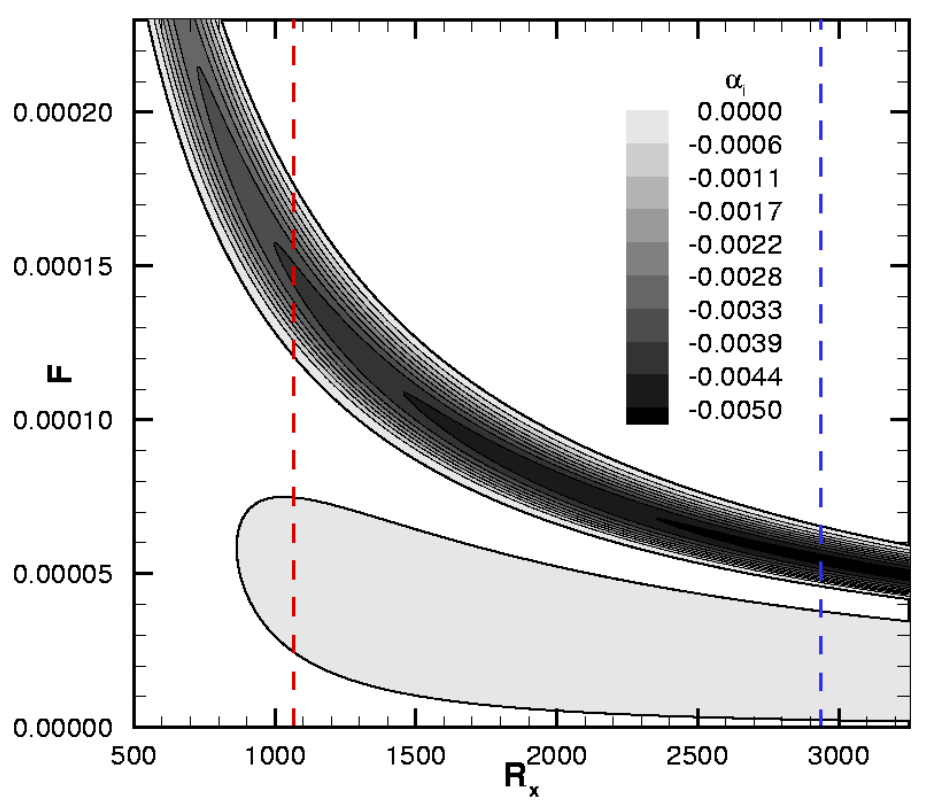

Figure 4.20: Contours of constant amplification rate $\alpha_{i}$ for 2D disturbances $(k=0)$. Obtained using Mack's LST solver Mack $(1965,1987)$. The low frequency band in the diagram corresponds to the first mode unstable region and the high frequency band represents the second mode unstable region. The vertical dashed lines in red and blue indicate the beginning and end of the computational domain used in the simulation. Isothermal wall. 
ure 4.10). As in figure 4.10 the first- and second-mode unstable regions are clearly visible in figure 4.20. The low-frequency band in the stability diagram corresponds to the first-mode unstable region and the high-frequency band represents the secondmode unstable region. Also note that the first-mode and second-mode unstable regions are separated by a stable region. The vertical dashed lines in red and blue indicate the start and end of the computational domain used in the simulations. In the simulations, the streamwise and wall-normal resolutions are chosen such that the relevant high-frequency two-dimensional second-mode waves are well resolved. To confirm that the resolution is sufficient the spatial growth rates, $\alpha_{i}$, and the streamwise wavenumber, $\alpha_{r}$, obtained from DNS are compared to LST results.

Streamwise wavelength and spatial growth rates obtained for three two-dimensional disturbance waves with different frequencies $(F=6.22 E-05, F=6.51 E-05$, and $F=6.81 E-05)$ from DNS are compared with LST in figure 4.21. As in the case of cone, the streamwise wavelength and spatial growth rates are calculated based on the wall pressure disturbance. The comparison resulted in similar outcomes as in the case of the cone. The streamwise wave number, $\alpha_{r}$, was found to be generally less sensitive to the criterion used to calculate it and therefore less sensitive to the non-parallel effects. Hence, the agreement between the streamwise wave number from DNS and LST is very good for all the three frequencies shown in figure 4.21. Close to the forcing location, however, similar to the cone results, the streamwise wave number, $\alpha_{r}$, and the spatial growth rate, $\alpha_{i}$, calculated for a flat-plate is strongly modulated by the superposition of damped or stable waves. This modulation is more pronounced for the spatial amplification rate, $\alpha_{i}$, than for the streamwise wave number, $\alpha_{r}$. Close to the location where disturbance waves exhibit maximum spatial growth there is a better agreement between the spatial amplification rate $\alpha_{i}$ from DNS and LST.

The wall-normal amplitude and phase distribution from the DNS for selected two-dimensional disturbances $(k=0)$ quantities at frequency $F=6.2246 E-05$ and streamwise position $x^{*}=0.437 m\left(R_{x}=2500\right)$ are compared to LST results in 

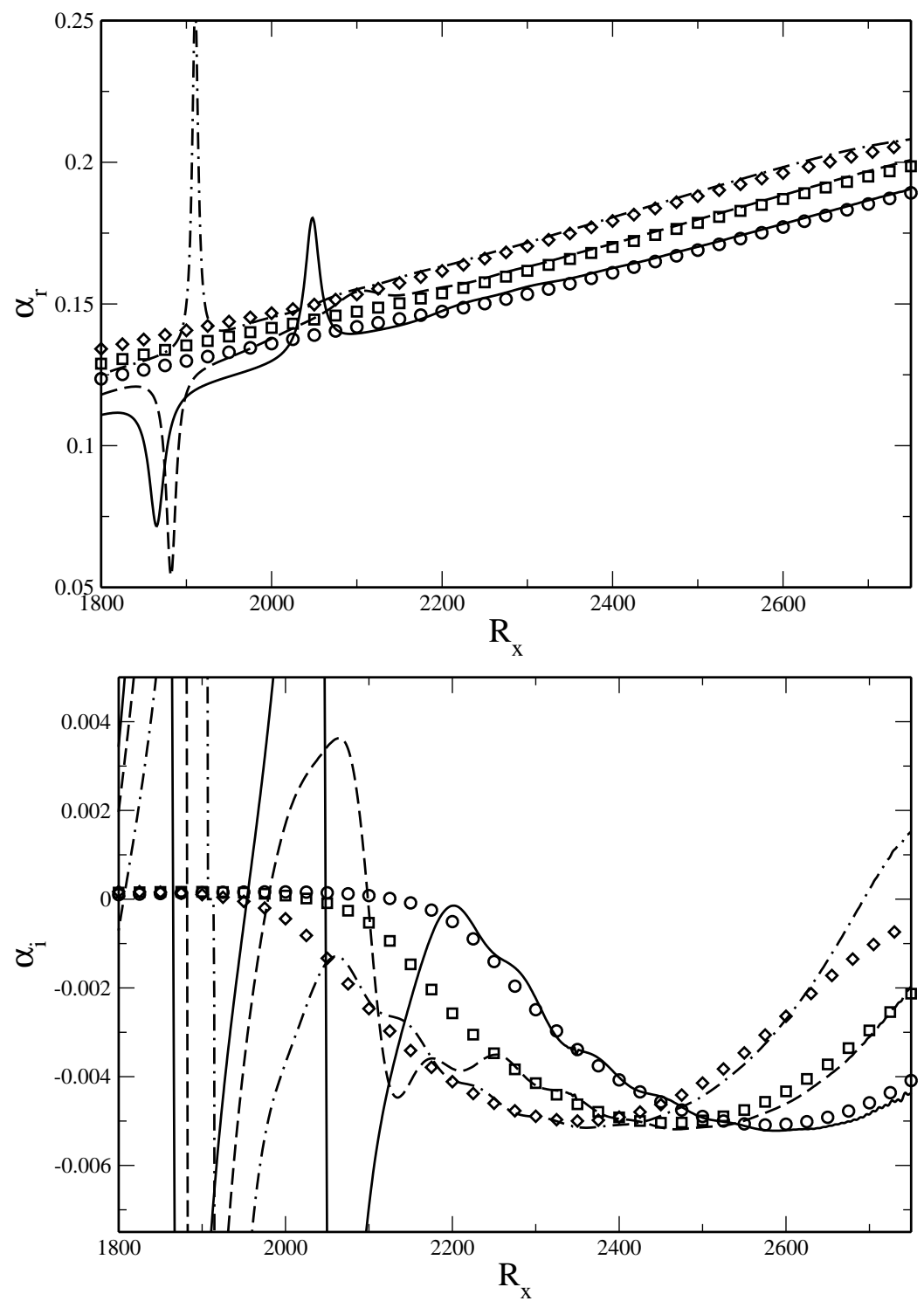

Figure 4.21: Comparison of streamwise wavelength $\left(\alpha_{r}\right)$ and spatial growth rates $\left(\alpha_{i}\right)$ for three 2D disturbance waves with different frequencies. Lines are from DNS and symbols are from LST. The frequencies are (a) $F=6.22 E-05(-, \circ)$ (b) $F=6.51 E-05(---, \square)$ and (c) $F=6.81 E-05(-.-.-, \diamond)$. LST results are computed using Mack's solver Mack $(1965,1987)$. Isothermal Wall. 

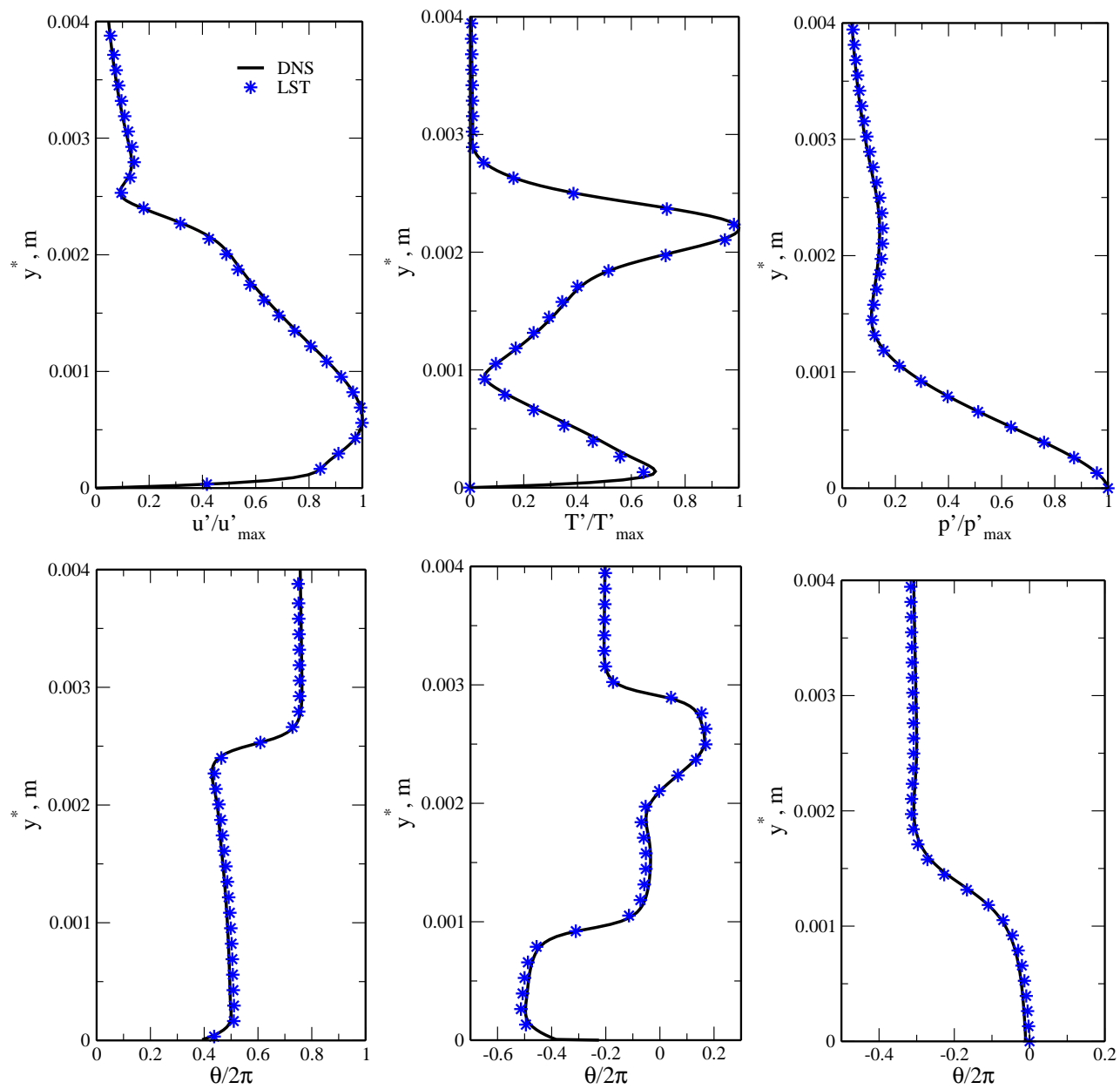

Figure 4.22: Comparison of wall-normal amplitude (top three plots) and phase distribution (bottom three plots) of the streamwise velocity, temperature and pressure disturbance to theoretical predictions from LST for frequency $F=6.2246 E-05$ and spanwise mode number $k=0$ at $R_{x}=2500$. LST results are computed using Mack's solver Mack $(1965,1987)$. Isothermal Wall. 
figure 4.22. The amplitude distributions for streamwise velocity, temperature and pressure disturbances obtained from DNS and LST are normalized by their respective maximum value. The excellent agreement between DNS and LST results in figure 4.22 is a strong indication that the simulations accurately capture the linear disturbance development.

The initial response of the flat-plate boundary layer flow to pulse forcing is illustrated in figure 4.23 by plotting contours of the instantaneous wall-pressure disturbance near the forcing location for several time instances. In figure $4.23 \mathrm{a}$, circular wave fronts can be seen emanating outward from the forcing location. Initially, the wave fronts within the packet are completely three-dimensional, an observation that is the same as for the cone. As the packet propagates downstream, however, the effects of stronger two-dimensional wave fronts can be observed near the centerline. This is expected because according to linear stability theory, two-dimensional waves experience higher streamwise amplification rates than oblique waves in hypersonic flat-plate boundary layers. Overall, the initial disturbance evolution in the flat-plate boundary layer is very similar to that observed for the isothermal cone in figure 4.4. It remains to see if the later developments are also going to be similar for the flat-plate and cone.

In order to answer that question, three snapshots of the flat-plate wave packet for later time instances are shown in figure 4.24. In figure 4.24a, three-dimensional wave fronts are seen to develop and lead the wave packet as it propagates downstream. Quite similar to what was observed for the cone boundary layer (see, figure 4.6a). These three-dimensional wave fronts are clearly visible in figure $4.24 \mathrm{~b}$ and seem to travel faster than the main wave packet. Therefore, these waves could be the stable fast-mode waves or acoustic waves, also observed in the case of a cone. It can be observed that the wave fronts close to the head of the main wave packet are predominantly two-dimensional. At the same time, wave fronts near the tail are three-dimensional (oblique). The packet experiences spatial and temporal growth as 

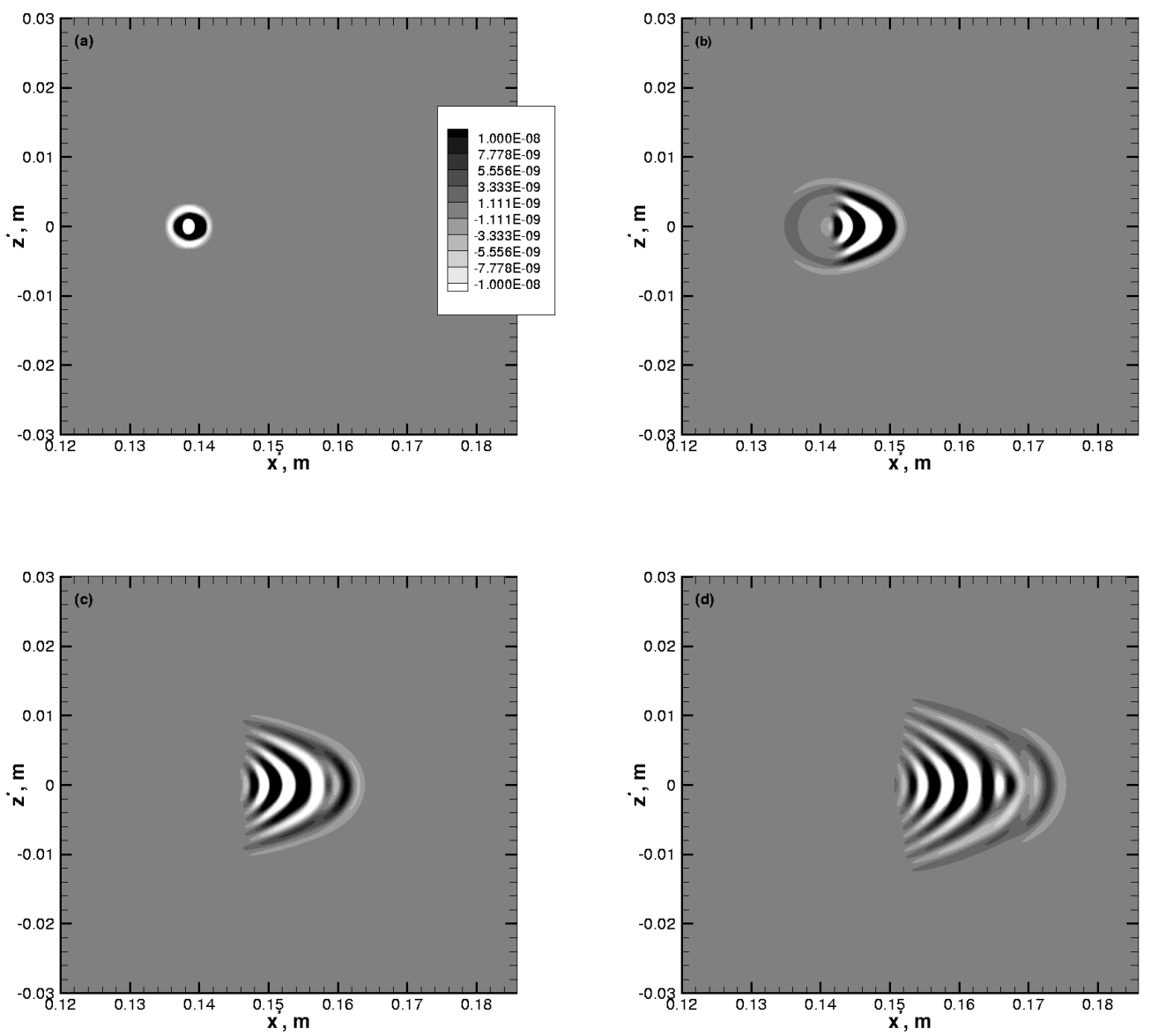

Figure 4.23: Snapshots of the linear wave packet showing the initial flow response to pulse forcing. Shown are contours of wall-pressure disturbance at (a) $t^{*}=0.006 \mathrm{~ms}$ (b) $t^{*}=0.017 \mathrm{~ms}$ (c) $t^{*}=0.029 \mathrm{~ms}$ and (d) $t^{*}=0.041 \mathrm{~ms}$. All the plots have the same contour levels. Isothermal Wall. 
it propagates towards the outflow boundary of the computational domain as seen in figure $4.24 \mathrm{c}$. It can be observed that the wave packet does not spread significantly in the spanwise direction, rather, it spreads over several wavelengths in the streamwise direction. Hence, the choice of the domain width was large enough for the flat-plate investigations. Also note the development of an arrowhead shape of the wave packet in figures $4.24 \mathrm{~b}$ and $\mathrm{c}$.

Despite the similarities in the shape during the initial disturbance evolution, the shape of a developed wave packet on the flat-plate is strikingly different from that of a sharp cone boundary layer discussed above. A comparison of linear wave packets for the flate-plate and a sharp cone boundary layer is shown in figure 4.25. As for the case of the flat-plate, the dominant wave fronts within the wave packet on a sharp cone are two-dimensional (or axisymmetric in the case of a cone). In addition, oblique waves seem to develop along the lateral sides of the wave packet in a sharp cone boundary layer. These oblique waves are not seen in the case of the flat-plate. The main reason for the different development is likely due to the fact that the wave angle decreases as the disturbances travel downstream in the case of a sharp cone. The decreasing wave angle is a consequence of the body divergence of the cone geometry causing the azimuthal wavelength of a particular wave to increase in the downstream direction. The waves within the wave packet on a sharp cone have a shorter streamwise wavelength than the waves in the flat-plate wave packet. This is due to the thinner boundary layer on the sharp cone in comparison to the boundary layer on the flat-plate.

In order to analyze the disturbance field, spectra were obtained from Fourier transformations of the wall-pressure disturbance using 500 Fourier modes in time. The broad disturbance spectrum produced by the short-duration localized pulse is shown in figure 4.26 in the spanwise mode number - frequency $\left(k-f^{*}\right)$ plane. A strong peak can be seen in the spectrum at frequency $f^{*} \sim 250 \mathrm{kHz}$, which corresponds to the pulse excitation. The frequency is directly related to the period of the localized 

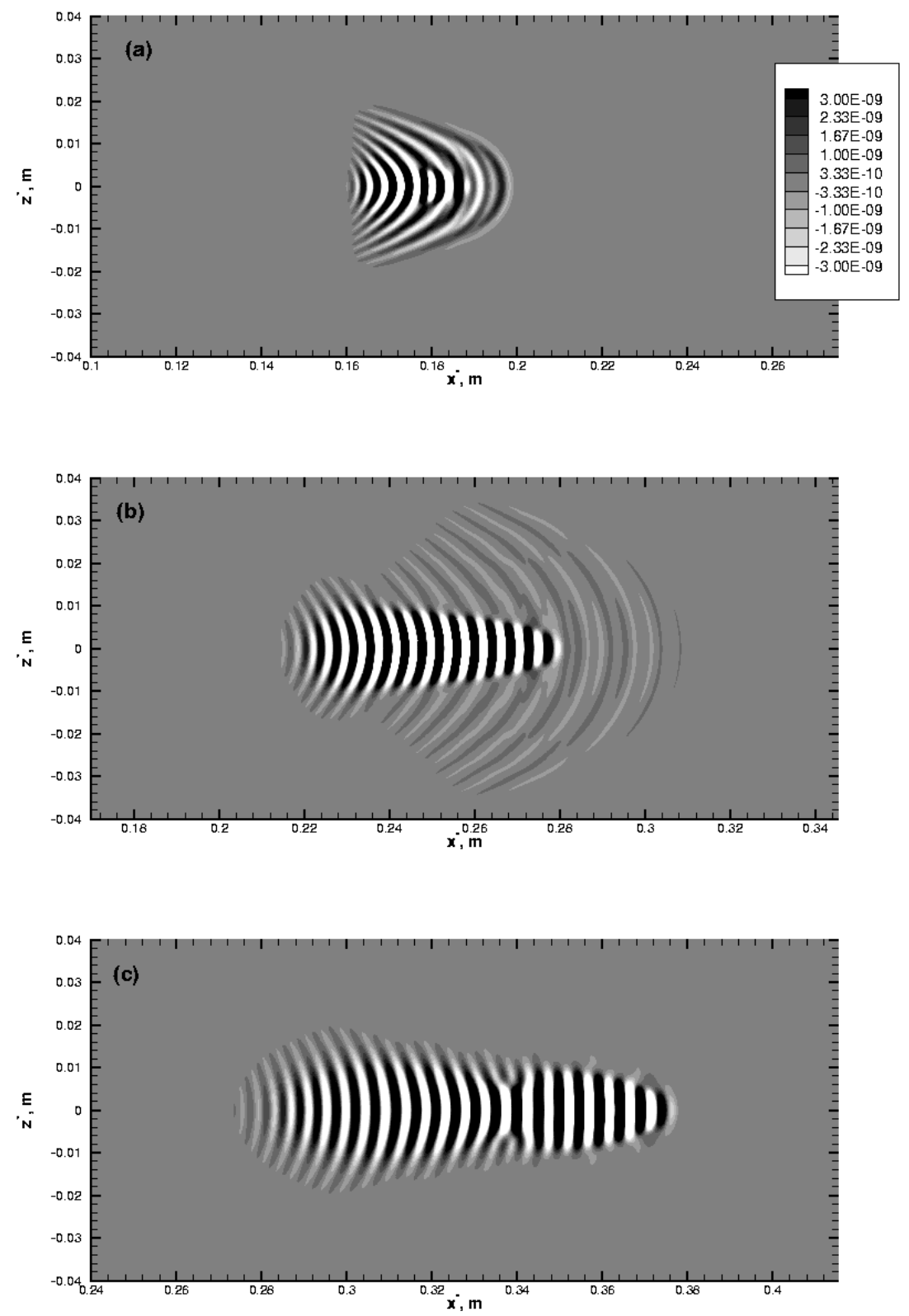

Figure 4.24: Snapshots of the linear wave packet illustrating its development in downstream direction. Shown are contours of wall-pressure disturbance at (a) $t^{*}=0.064 \mathrm{~ms}(\mathrm{~b}) t^{*}=0.174 \mathrm{~ms}$ and $(\mathrm{c}) t^{*}=0.291 \mathrm{~ms}$. All the plots have the same contour levels. Isothermal Wall. 

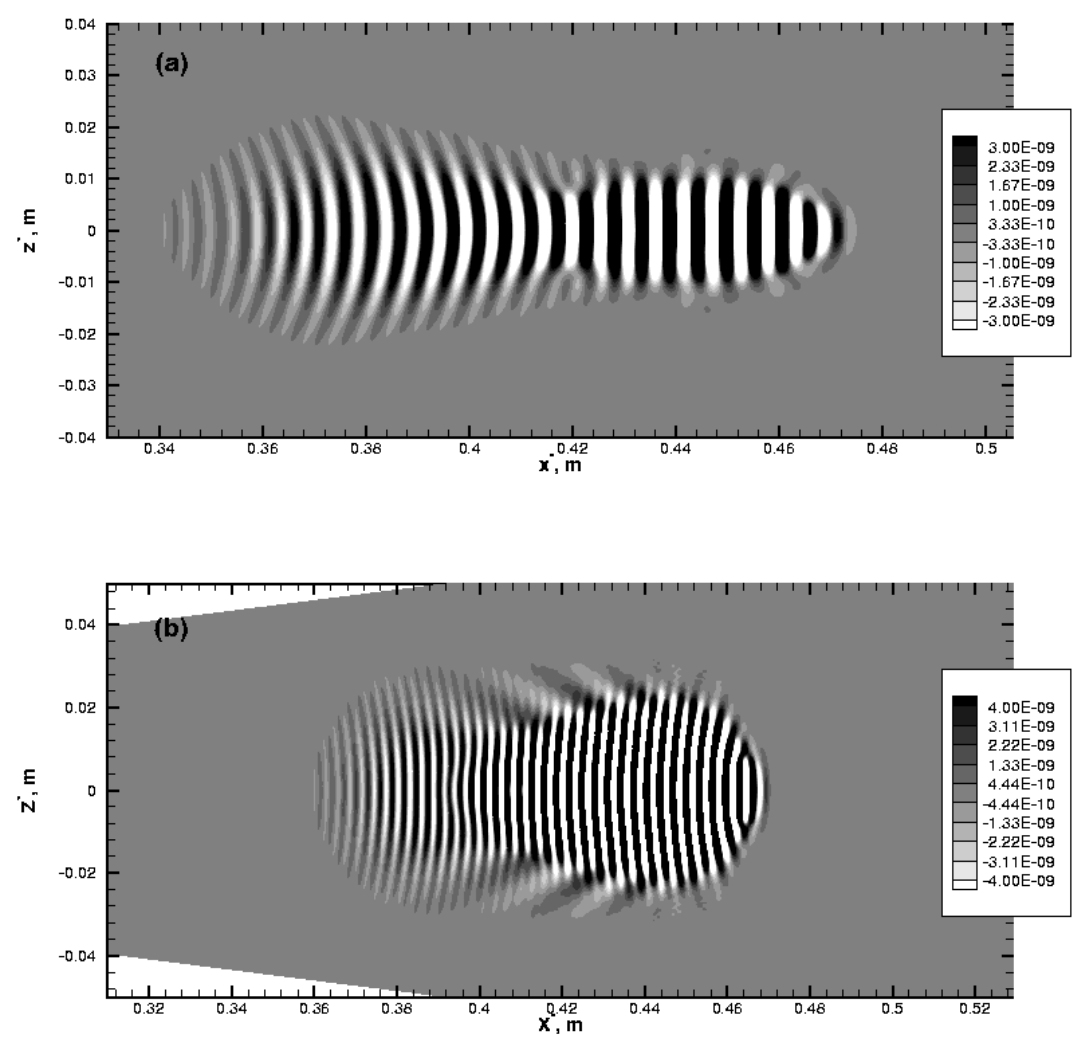

Figure 4.25: Comparison of linear wave packets in a flat-plate and a sharp cone boundary layer. Shown are contours of wall-pressure disturbance for (a) a flat-plate and (b) a sharp cone at $t^{*}=0.4 \mathrm{~ms}$. Isothermal Wall.

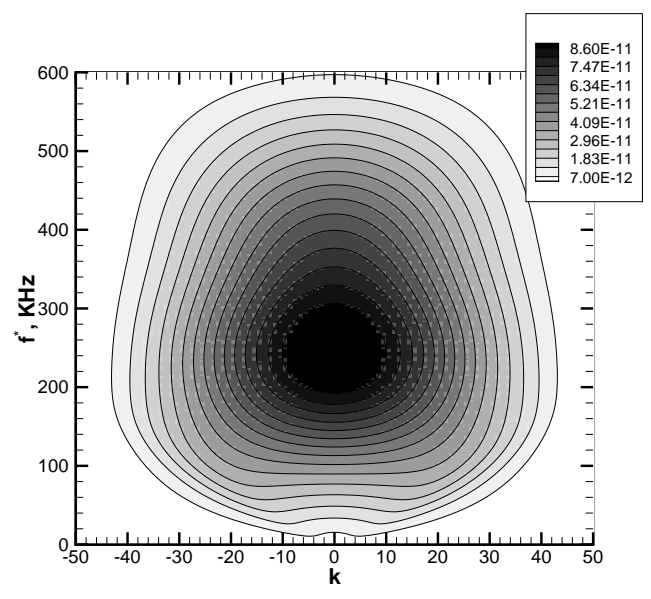

Figure 4.26: Disturbance spectrum in the spanwise mode number - frequency $\left(k-f^{*}\right)$ plane obtained at the forcing location $\left(x^{*}=0.138 m\right)$ for the simulation with low forcing amplitude. Isothermal wall. 

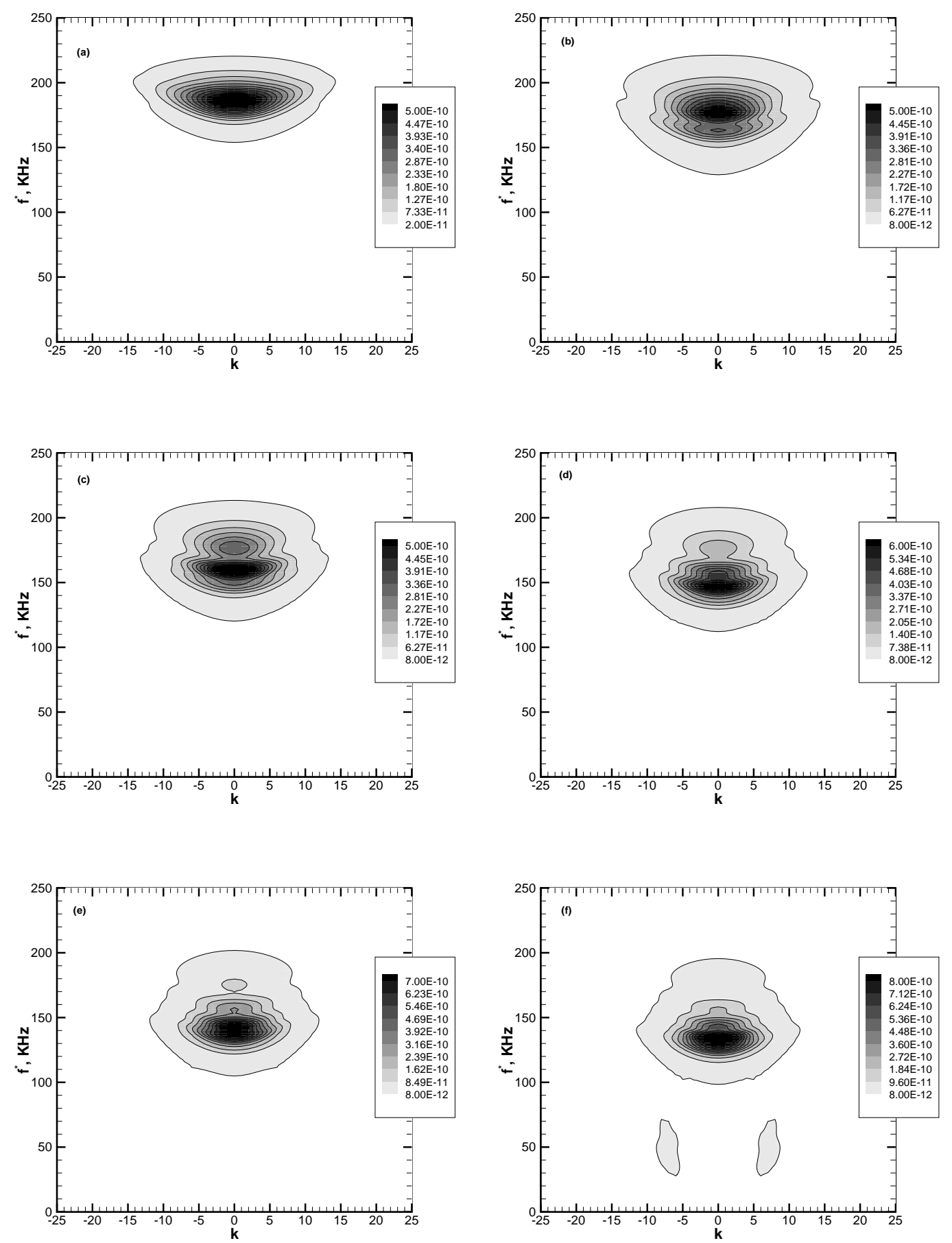

Figure 4.27: Disturbance spectrum in the spanwise mode number - frequency $\left(k-f^{*}\right)$ plane for the simulation with low forcing amplitude obtained from wall-pressure at different streamwise positions. (a) $x^{*}=0.250 \mathrm{~m}$, (b) $x^{*}=0.300 \mathrm{~m}$, (c) $x^{*}=0.350 \mathrm{~m}$, (d) $x^{*}=0.400 m$, (e) $x^{*}=0.450 m$ and (f) $x^{*}=0.500 m$. Isothermal Wall. 
pulse disturbance introduced into the boundary layer, it was reduced for the flat-plate (when compared to the cone, see, figure 4.16a) because the flat-plate boundary layer is thicker than the one on the cone, and, as a result, the dominant second-mode waves on the flat-plate has a lower frequency when compared to the dominant second-mode waves on a cone. The streamwise development of the disturbance spectrum is shown in figure 4.27. As expected, at all streamwise positions $\left(x^{*}=0.250 m, x^{*}=0.300 m\right.$, $x^{*}=0.350 m, x^{*}=0.400 m, x^{*}=0.450 m$ and $\left.x^{*}=0.500 m\right)$, the spectra exhibit a maximum at the spanwise mode number $k=0$, because two-dimensional disturbances have a higher streamwise amplification rate than oblique waves. Lower-frequency first-mode oblique disturbances with a peak at approximately $k \pm 7$ and $f^{*} \sim 50 \mathrm{kHz}$ also become visible. Overall, the peak amplitude remains fixed at $k=0$ but shifts to lower frequencies in the downstream direction as the boundary layer becomes thicker. For the flat-plate, the disturbance spectrum does not spread over higher spanwise mode numbers (oblique waves) to the extent it does for a sharp cone (see figure 4.17). This might be an effect of the decreasing wave angle in the case of a sharp cone.

\subsection{Weakly Nonlinear Wave Packet}

In the previous section the linear development of the wave packet was studied in detail by forcing the flow with a low-amplitude pulse. The dominant waves within the resulting linear wave packet were identified as the second-mode two-dimensional or axisymmetric disturbance waves. In addition, weaker first-mode oblique waves were also observed on the lateral sides of the wave packet. However, the main objective of this research is to identify the dominant nonlinear mechanisms for hypersonic boundary layers at Mach 6. Therefore, simulations were performed with a moderate forcing amplitude ( $0.5 \%$ of the free-stream velocity) to investigate the weakly nonlinear transition regime. The forcing amplitude was chosen such that the wave packet at first grows linearly before it reaches the weakly nonlinear regime. Both isothermal 
$\left(T_{w}=300\right)$ and adiabatic $\left(T_{w}=T_{a w}\right)$ wall boundary conditions are considered to study the effect of wall temperature on the early nonlinear regime.

\subsubsection{Development of the wave packet}

The temporal and spatial evolution of the centerline wall-pressure disturbance amplitude is shown in figures 4.28 and 4.29 for the weakly nonlinear wave packets. As for the linear wave packets, in these figures a scale given at the bottom indicates the disturbance amplitude. As observed for the linear wave packet, the scale for the isothermal wall case has a larger value compared to the one for the adiabatic wall. This is again attributed to the higher growth rates of the second-mode wave in the isothermal wall case (destabilizing effect on the second-mode waves). As a result, the waves within the weakly nonlinear wave packet for the isothermal wall case reached large amplitudes compared to the adiabatic wall case. The initial development of the packets in figures 4.28 and 4.29 looks similar to the linear wave packets shown in figures 4.2 and 4.3, respectively. For the isothermal wall case, the packet looks linear up to $x^{*}=0.35 m$ and for the adiabatic wall case, up to $x^{*}=0.45 m$. Later the wave packet becomes strongly deformed, caused by nonlinear interactions. The wave packet appears to be splitting into two parts as it propagates downstream, as indicated by the "kink" in the envelope of the wave packet. The weakly nonlinear wave packet for the isothermal wall case starts to show symptoms of nonlinear interactions slightly upstream of the packet for the adiabatic wall case. This is again due to the higher growth rates of second-mode waves in the isothermal wall boundary layer.

As discussed above, the initial evolution of the weakly nonlinear wavepacket is very similar to the linear wave packet discussed in the previous section. However, the later developments will be different due to nonlinear effects. In order to visualize the later development, three snapshots of the weakly nonlinear wave packets are shown in figures 4.30 and 4.31 for the isothermal and adiabatic wall case. These snapshots 


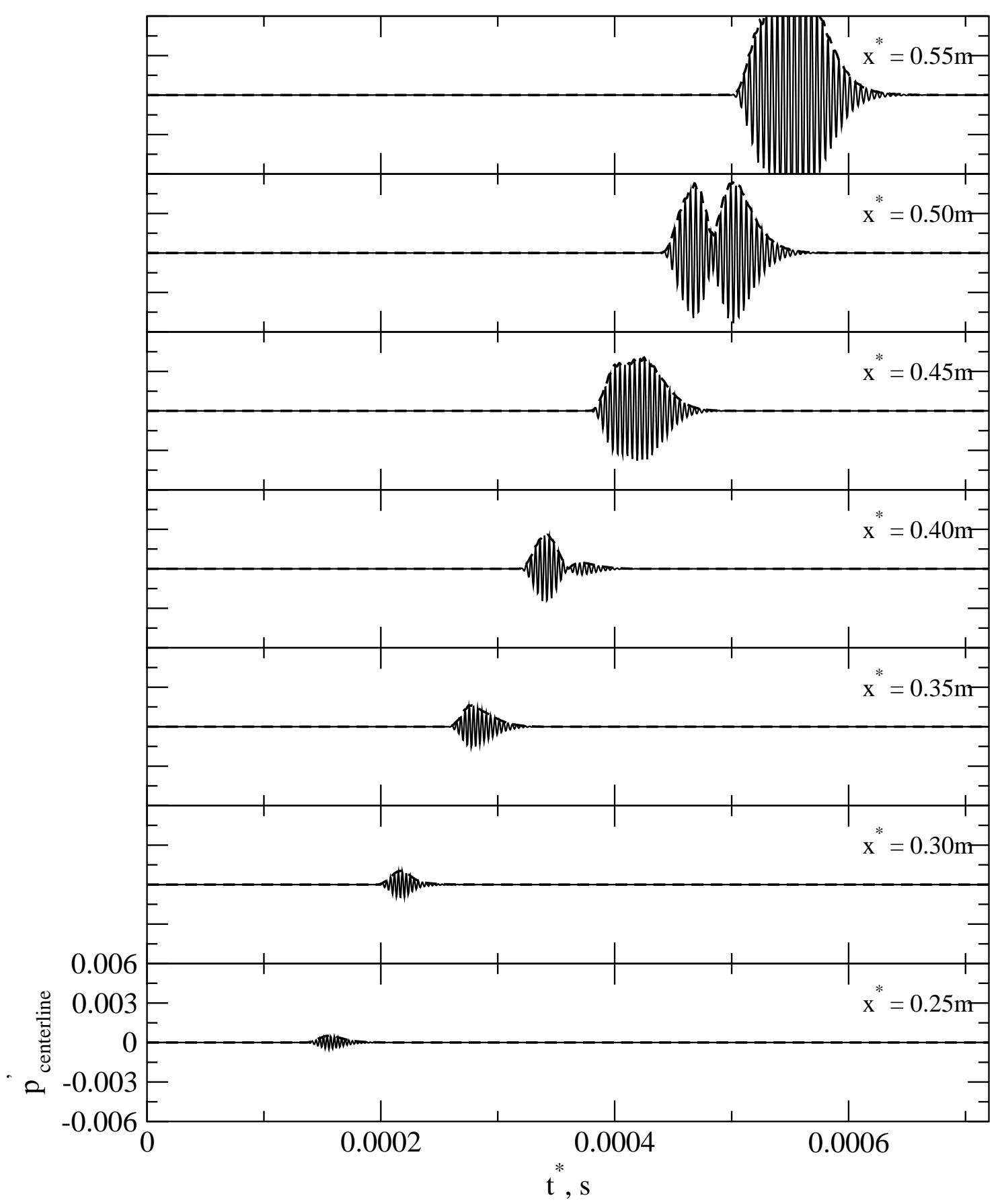

Figure 4.28: Temporal and spatial evolution of wall-pressure disturbance amplitude along the centerline for the weakly nonlinear wave packet. Isothermal Wall. 


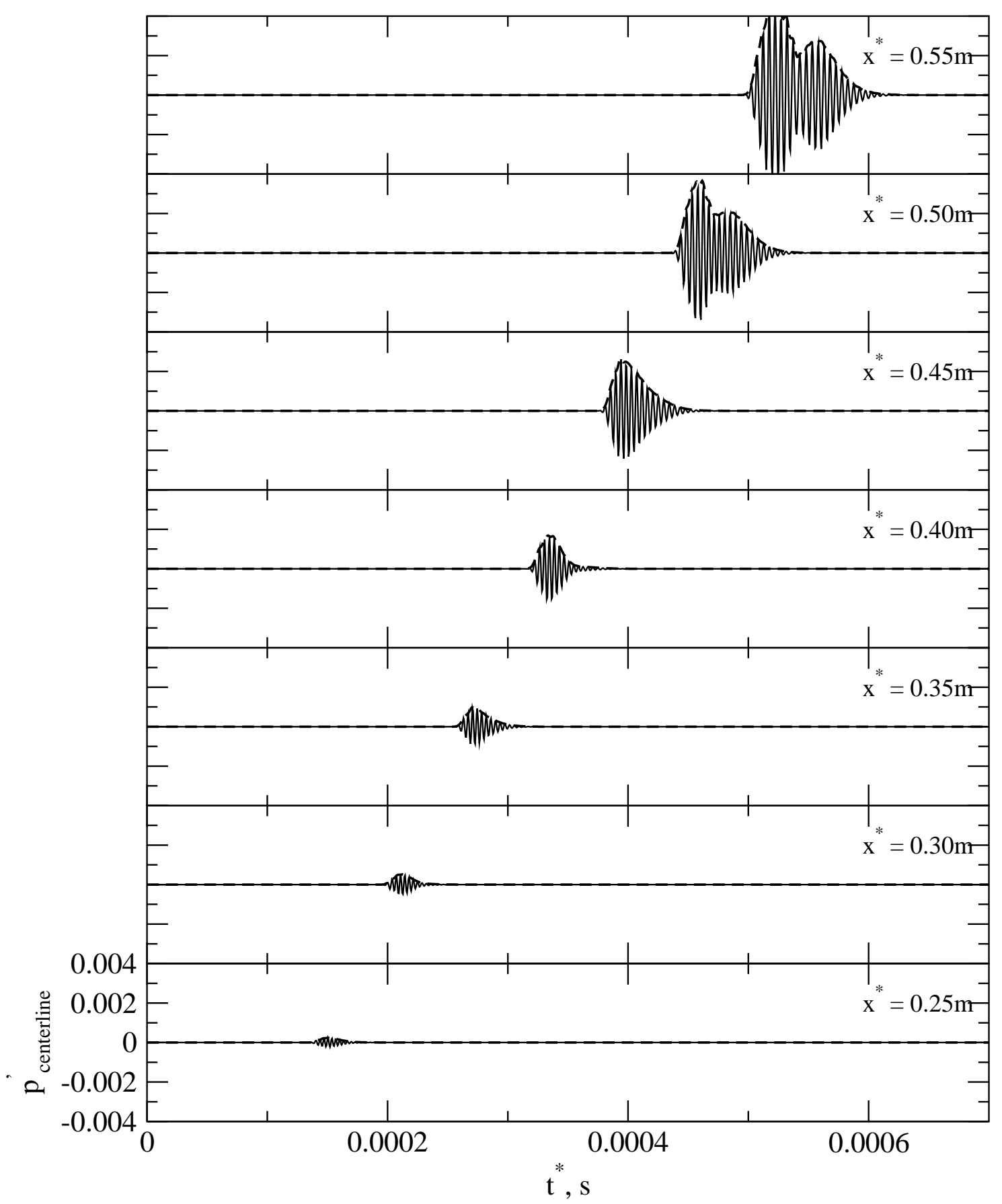

Figure 4.29: Temporal and spatial evolution of wall-pressure disturbance amplitude along the centerline for the weakly nonlinear wave packet. Adiabatic wall. 
are for $t^{*}=0.40 \mathrm{~ms}, t^{*}=0.46 \mathrm{~ms}$ and $t^{*}=0.51 \mathrm{~ms}$. In figures $4.30 \mathrm{a}$ and 4.31 for $t^{*}=0.40 \mathrm{~ms}$ the wave packet already appears slightly altered close to the tail by nonlinear effects. Circular wave fronts of the acoustic waves propagating in front of the main wave packets are clearly visible. At $t^{*}=0.46 \mathrm{~ms}$ the axisymmetric waves at the tail of the wave packet in figures $4.30 \mathrm{~b}$ and $4.31 \mathrm{~b}$ are modulated in the azimuthal direction. Later at $t^{*}=0.46 \mathrm{~ms}$, these waves are more strongly modulated in the azimuthal direction. This may be caused by nonlinear interaction of the axisymmetric waves with oblique waves. In addition to that, changes can also be observed in the center of the weakly nonlinear wave packets. By now the acoustic waves propagating in front of the main wave packets have weakened as these stable modes continue to decay while travelling downstream.

\subsubsection{Disturbance Field}

In figures 4.32 and 4.33, wall-pressure disturbance spectra are shown in the azimuthal wave number vs frequency plane for several streamwise positions. The disturbance spectra obtained for $x^{*}=0.25 m$ (see figures $4.32 \mathrm{a}$ and $4.33 \mathrm{a}$ ) look very similar to those of the linear wave packet (see figures $4.17 \mathrm{a}$ and $4.18 \mathrm{a}$ ), which confirms that initially the wave packet develops linearly. The disturbance spectrum for $x^{*}=0.35 \mathrm{~m}$ (figures $4.32 \mathrm{~b}$ and $4.33 \mathrm{~b}$ ) indicates weak nonlinear interactions as the spectrum starts to deform. For $x^{*}=0.45 m$ (figures $4.32 \mathrm{c}$ and $4.33 \mathrm{c}$ ) the spectrum broadens with respect to the azimuthal wave number range and additional frequency bands develop. In addition, low-frequency first-mode oblique waves could also be identified.

Farther downstream at $x^{*}=0.55 \mathrm{~m}$ the spectrum has spread further over higher azimuthal mode numbers, and continues to develop other frequency bands. This is a strong indication of nonlinear interactions between the dominant axisymmetric waves and oblique waves. Bands of low-frequency waves show up in the spectrum, which may have been generated nonlinearly. This development intensifies in the down- 

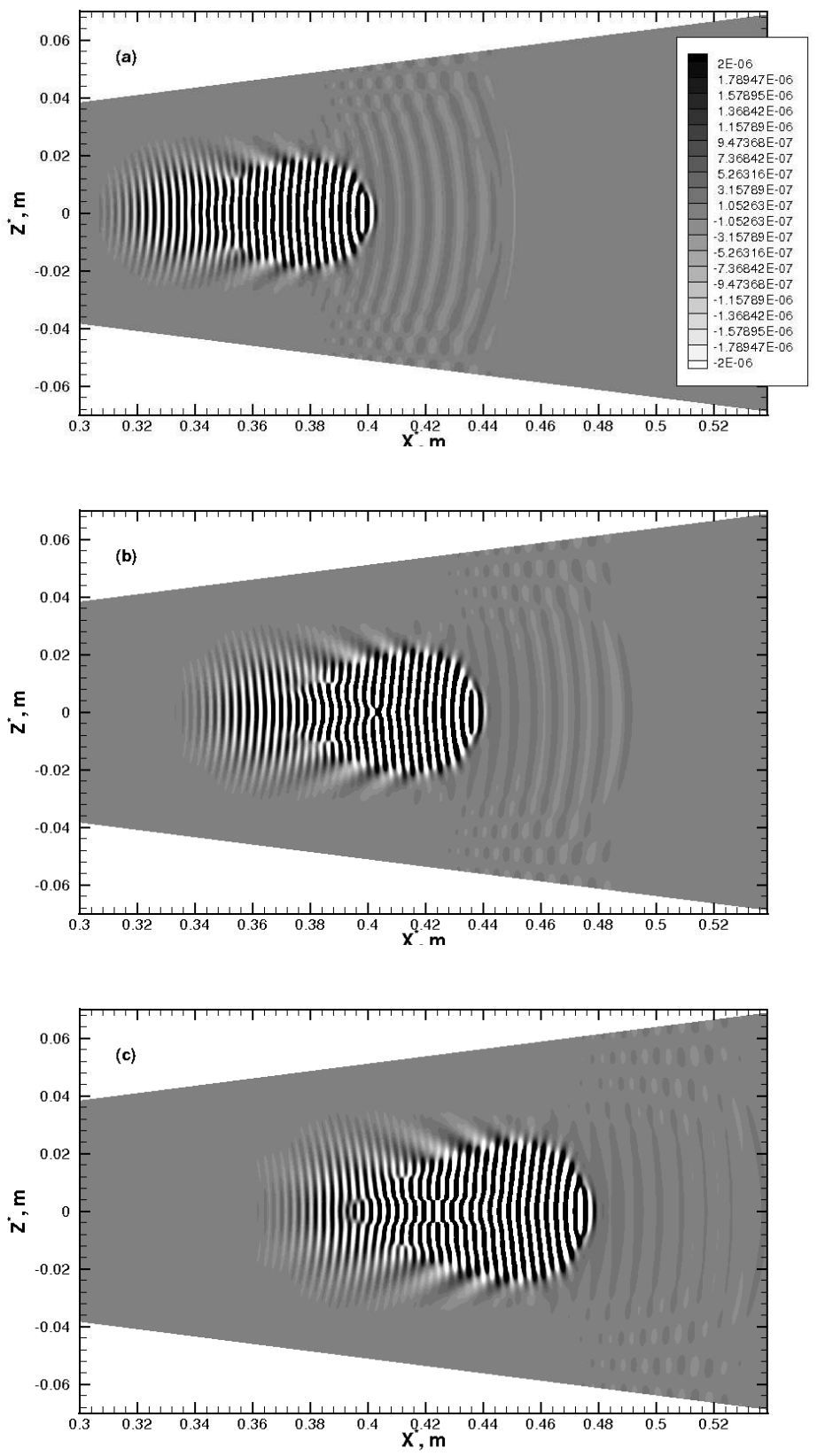

Figure 4.30: Snapshots of the weakly nonlinear wave packet illustrating its development in downstream direction. Shown are contours of wall-pressure disturbance on the unrolled cone surface at (a) $t^{*}=0.40 \mathrm{~ms}$, (b) $t^{*}=0.46 \mathrm{~ms}$ and (c) $t^{*}=0.51 \mathrm{~ms}$. All the plots have the same contour levels. Isothermal Wall. 

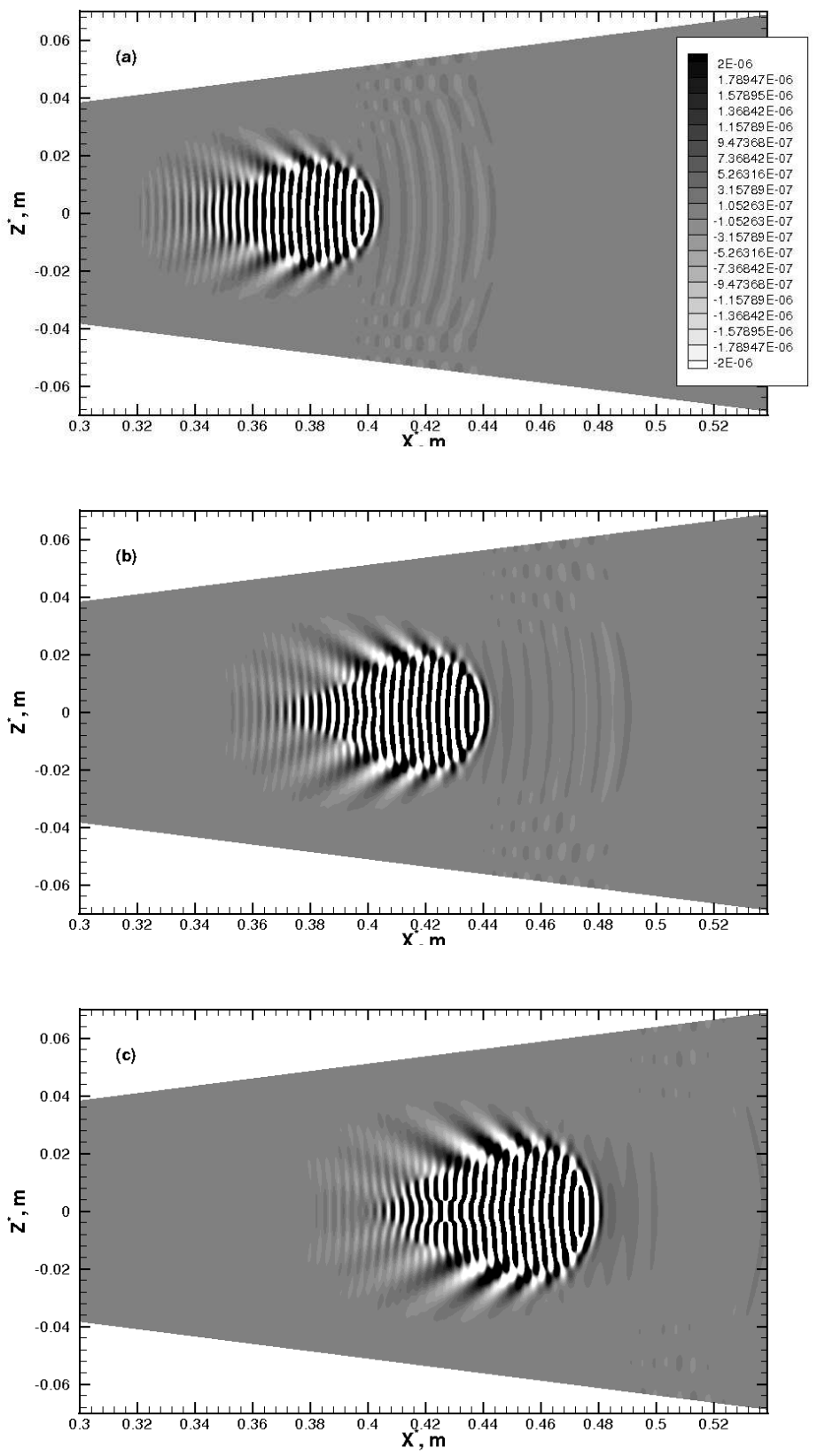

Figure 4.31: Snapshots of the weakly nonlinear wave packet illustrating its development in downstream direction. Shown are contours of wall-pressure disturbance on the unrolled cone surface at (a) $t^{*}=0.40 \mathrm{~ms}$, (b) $t^{*}=0.46 \mathrm{~ms}$ and (c) $t^{*}=0.51 \mathrm{~ms}$. All the plots have the same contour levels. Adiabatic Wall 

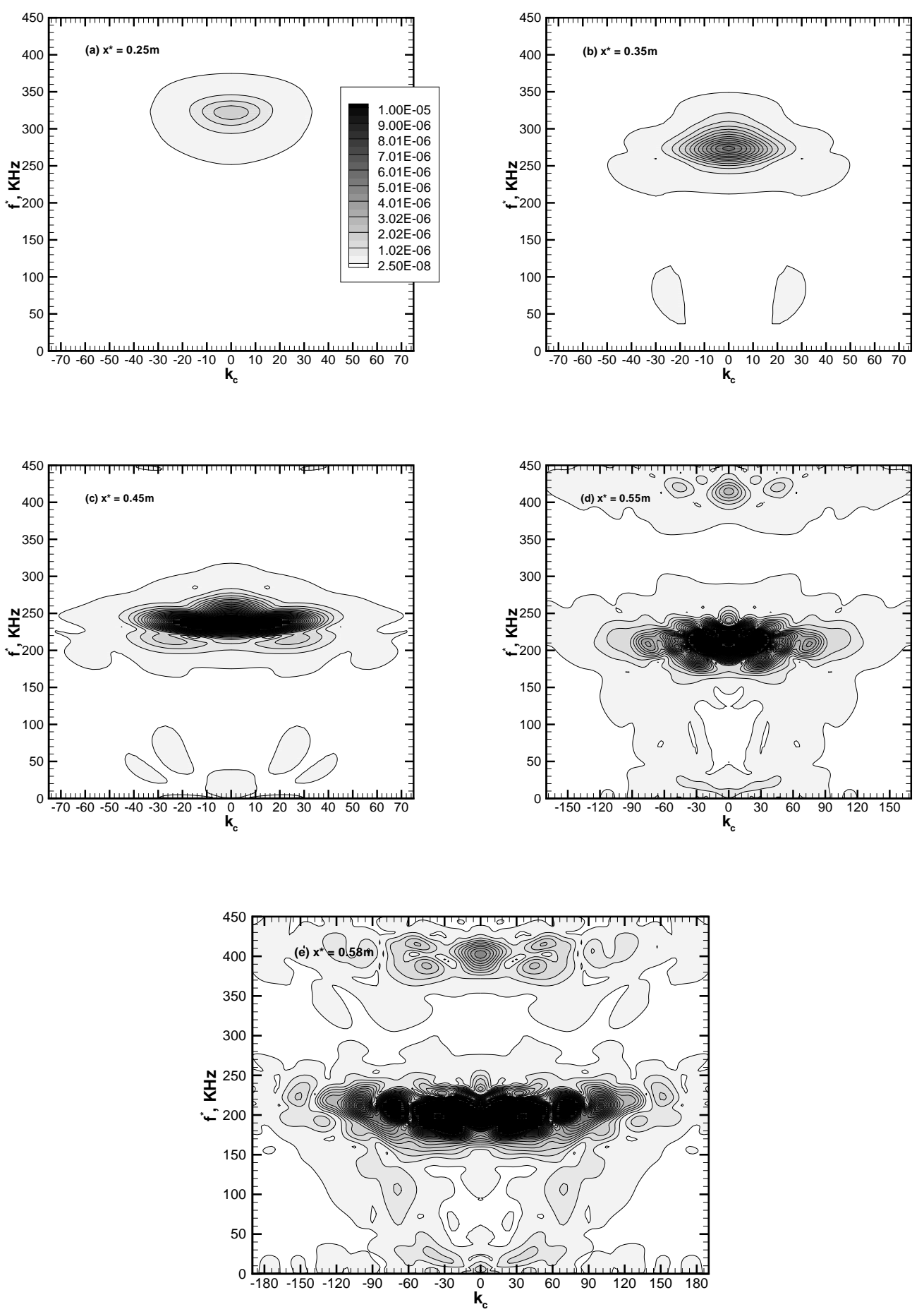

Figure 4.32: Disturbance spectrum in the azimuthal mode number - frequency $\left(k_{c}-\right.$ $\left.f^{*}\right)$ plane, obtained from wall-pressure at different streamwise positions. (a) $x^{*}=$ $0.250 m,(\mathrm{~b}) x^{*}=0.350 \mathrm{~m}$, (c) $x^{*}=0.450 \mathrm{~m}$, (d) $x^{*}=0.550 \mathrm{~m},(\mathrm{e}) x^{*}=0.580 \mathrm{~m}$. All the plots have the same contour levels. Isothermal wall. 

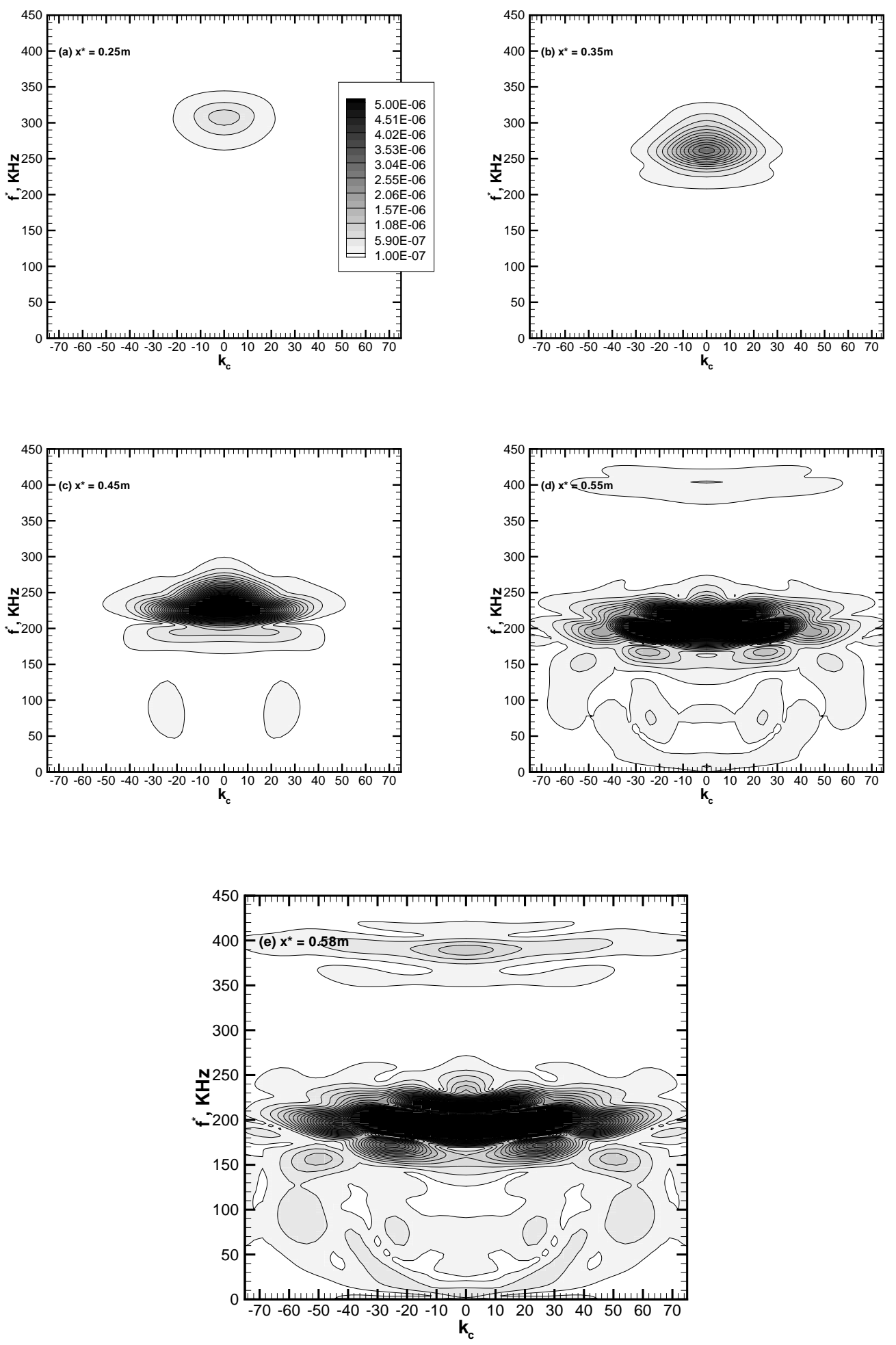

Figure 4.33: Disturbance spectrum in the azimuthal mode number - frequency $\left(k_{c}-\right.$ $f^{*}$ ) plane, obtained from wall-pressure at different streamwise positions. (a) $x^{*}=$ $0.250 \mathrm{~m},(\mathrm{~b}) x^{*}=0.350 \mathrm{~m}$, (c) $x^{*}=0.450 \mathrm{~m}$, (d) $x^{*}=0.550 \mathrm{~m}$, (e) $x^{*}=0.580 \mathrm{~m}$. All the plots have the same contour levels. Adiabatic wall. 
stream direction as can be observed by comparing the spectra at locations farther downstream with those at locations farther upstream. The spectrum for $x^{*}=0.580 \mathrm{~m}$ in figures $4.32 \mathrm{e}$ and $4.33 \mathrm{e}$ shows strong secondary peaks at the fundamental frequency $\left(f^{*} \approx 200 K H z\right)$ for larger azimuthal wave numbers. This development may be an indication of a fundamental resonance mechanism. Secondary peaks are observed for very high azimuthal mode numbers $\left(k_{c} \approx 40-180\right.$, see figure $\left.4.32 \mathrm{e}\right)$ for the isothermal wall case and were only observed for moderately high azimuthal mode numbers $\left(k_{c} \approx 40-70\right.$, see figure $\left.4.33 \mathrm{e}\right)$ for the adiabatic wall case. In addition secondary peaks are observed in figures $4.32 \mathrm{e}$ and $4.33 \mathrm{e}$ at approximately half the frequency $\left(f^{*} \approx 100 \mathrm{KHz}\right.$ ) of the high-amplitude frequency band for azimuthal mode number $k_{c} \pm 70$ for the isothermal wall case and for azimuthal mode number $k_{c} \pm 55$ for the adiabatic wall case, which would be an indication of a subharmonic resonance. However, the spectra in figures $4.32 \mathrm{e}$ and $4.33 \mathrm{e}$ suggests that fundamental resonance is much stronger than subharmonic resonance for both isothermal and adiabatic wall cases. Strong peaks were also observed for low-wave-number (shallow wave angle) second-mode oblique waves for both isothermal and adiabatic wall cases, which indicates a possible presence of an oblique breakdown mechanism. Higher harmonics $\left(f^{*} \approx 400 \mathrm{KHz}\right)$ of the high amplitude frequency band $\left(f^{*} \approx 200 \mathrm{KHz}\right)$ are also visible in figures $4.32 \mathrm{e}$ and $4.33 \mathrm{e}$. Note that strong second harmonic disturbances were also observed in the experiments by Stetson et al. (1983). Overall, the streamwise development of the disturbances for the isothermal and adiabatic wall cases are qualitatively similar.

\subsubsection{Comparison with flat plate}

The weakly nonlinear transition regime of a Mach 6 cone boundary layer have been investigated in the previous sections using a medium-amplitude localized pulse disturbance. The response of the flow to the medium-amplitude pulse indicated the 
presence of a fundamental and subharmanic resonance mechanism. Strong peaks were also observed for low-wave-number second-mode oblique waves, which indicate a possible presence of an oblique breakdown mechanism. In order to study the effect of geometry on these nonlinear mechanisms identified as the dominant mechanisms for a sharp cone, a weakly nonlinear wave packet simulation was performed for a flat-plate boundary layer. As for the linear wave packet in a flat-plate boundary layer discussed in section 4.2.3, the boundary layer edge values matched those of the cone and an isothermal wall boundary condition $\left(T_{w}=300 K\right)$ is used. The forcing amplitude was chosen such that the wave packet grows linearly before it enters the weakly nonlinear regime.

The temporal and spatial evolution of the centerline wall-pressure disturbance amplitude is shown in figure 4.34. As for the linear wave packet, in this figure a scale given for the bottom signal indicates the disturbance amplitude. The initial development of the packet looks similar to the linear wave packet shown in figure 4.19. Later the wave packet becomes strongly deformed, caused by nonlinear interactions (see signal at $x^{*}=0.5 \mathrm{~m}$ ). The wave packet appears to be splitting into two parts as it propagates downstream, as indicated by the "kink" in the envelope.

In order to visualize the later development, four snapshots of the nonlinear wave packet are shown in figure 4.35. The snapshots are for $t^{*}=0.17 \mathrm{~ms}, t^{*}=0.29 \mathrm{~ms}$, $t^{*}=0.41 \mathrm{~ms}$ and $t^{*}=0.52 \mathrm{~ms}$. In figure $4.35 \mathrm{a}$ for $t^{*}=0.17 \mathrm{~ms}$ three-dimensional wave fronts are seen to lead the main wave packet. These waves are also seen in figure 4.35b. They seem to travel slightly faster than the main wave packet. Note that these acoustic waves were also seen in the case of the linear wave packet. As for the linear case the wave fronts close to the head of the main wave packet are two-dimensional and the wave fronts near the tail are three-dimensional (oblique). In figure 4.35c the wave packet appears altered by nonlinear effects. The two-dimensional wave fronts close to the center of the wave packet looks modulated in the spanwise direction. This might be caused by nonlinear interaction of the two-dimensional waves with 


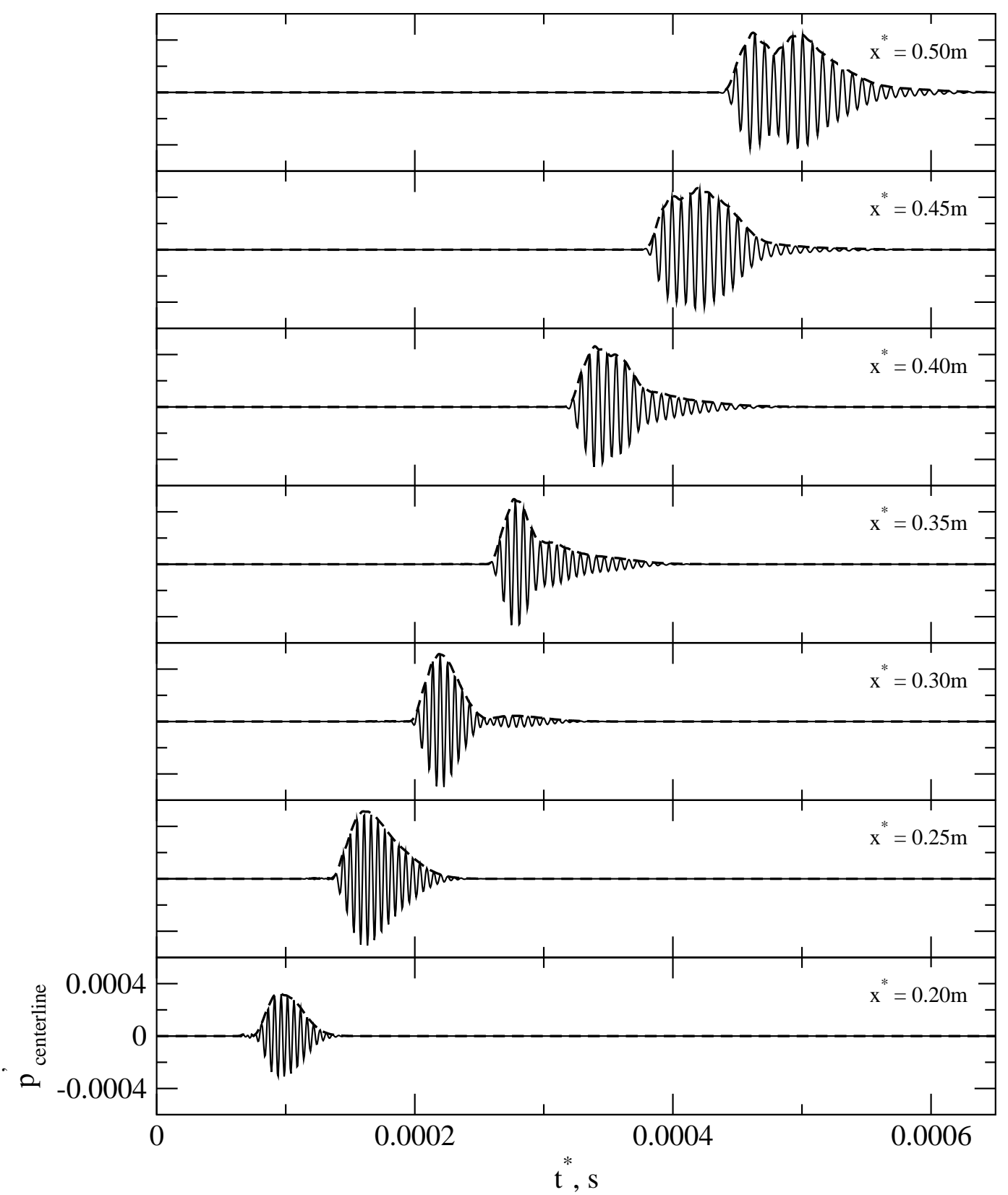

Figure 4.34: Temporal and spatial evolution of wall-pressure disturbance amplitude along the centerline for the nonlinear wave packet. Solid lines $(-)$ represent the time signal and dashed lines (- - ) its envelope. Isothermal wall. 
oblique waves. Furthermore, the packet seem to have spread more in the streamwise direction. Then for $t^{*}=0.52 \mathrm{~ms}$, in figure $4.35 \mathrm{~d}$, the packet looks more developed and has the shape of an arrowhead. Three-dimensional (oblique) wave fronts can be seen along the lateral sides of the wave packet. In addition, the wave fronts close to the center and tail of the packet look more oblique than in the earlier snapshot.

In figure 4.36 wall-pressure disturbance spectra are shown in the spanwise mode number vs frequency plane for several streamwise positions. The disturbance spectra obtained for $x^{*}=0.250 m$ (see figure 4.36 a) look very similar to those of the linear wave packet (see figure 4.27 a), which confirms that initially the wave packet develops linearly. The disturbance spectrum for $x^{*}=0.300 m$ (figure 4.36b) indicates weak nonlinear interactions as the spectrum starts to broaden with respect to the spanwise mode number range and additional frequency bands develop. For $x^{*}=0.350 \mathrm{~m}$ the spectrum has spread further over higher spanwise mode numbers, and continues to develop other frequency bands. This is an indication of nonlinear interactions between the dominant two-dimentional waves and oblique waves. In addition, lower frequency first-mode oblique waves could also be identified along with other lower frequency waves, which may have been generated nonlinearly. This development intensifies in the downstream direction as can be observed by comparing the spectra at locations farther downstream with those from locations farther upstream. The spectrum for $x^{*}=0.500 \mathrm{~m}$ in figure $4.36 \mathrm{f}$ shows strong peaks at the fundamental frequency $f^{*} \approx$ $140 \mathrm{KHz}$ for higher spanwise mode numbers upto $k \approx 20$. This development may be an indication of a fundamental resonance mechanism. Higher harmonics $f^{*} \approx$ $280 \mathrm{KHz}$ of the high-amplitude frequency band $f^{*} \approx 140 \mathrm{KHz}$ are also visible in figures $4.36 \mathrm{e}$ and f. Note again, that strong second harmonic disturbances were also observed in the experiments by Stetson et al. (1983). In addition, secondary peaks are observed in figure 4.36f at approximately half the frequency of the high-amplitude frequency band for spanwise mode number $k \pm 15$, which would be an indication of a subharmonic resonance. However, the spectrum in figure $4.36 \mathrm{f}$ indicates that 

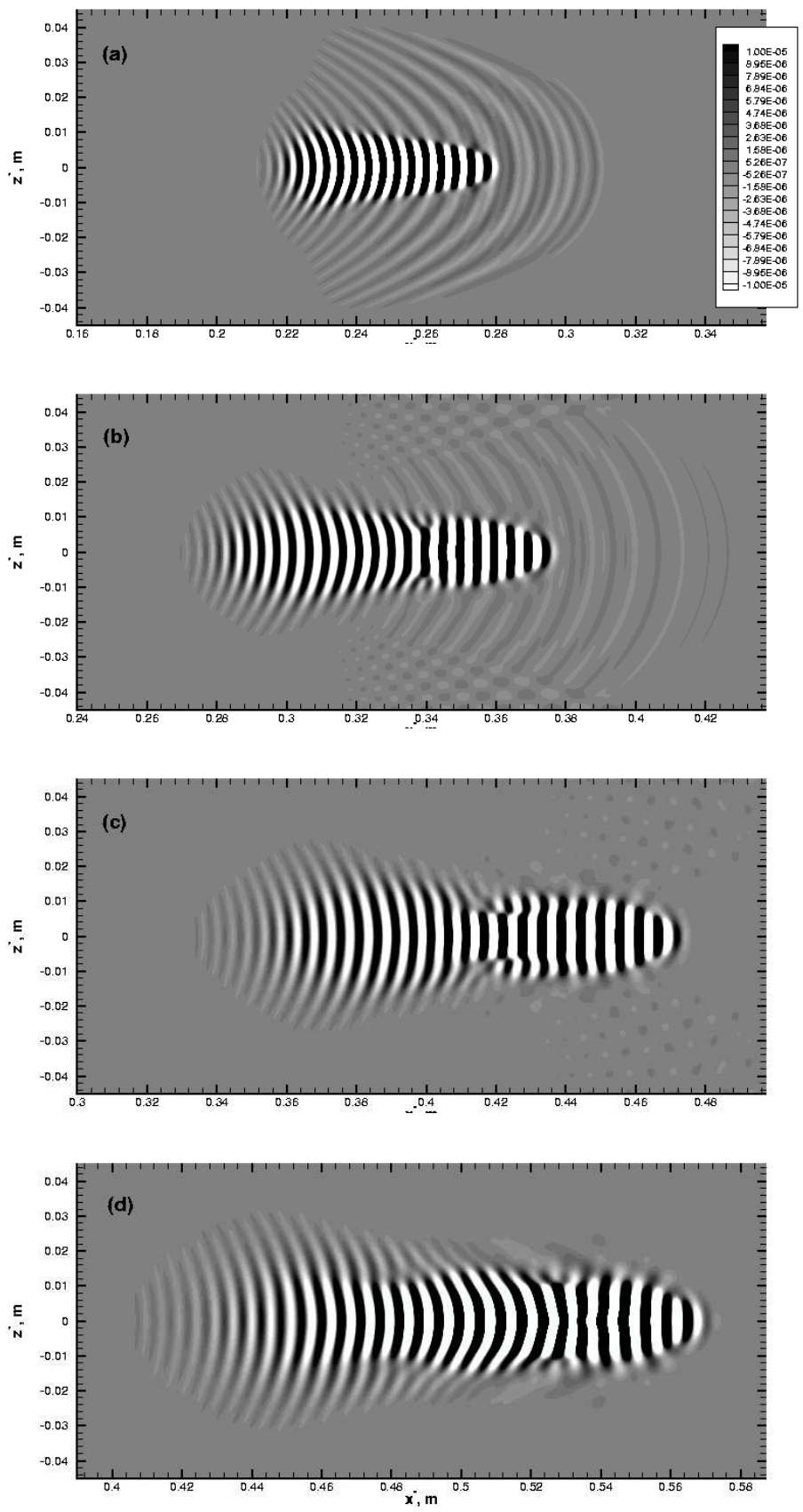

Figure 4.35: Snapshots of the nonlinear wave packet illustrating its development in downstream direction. Shown are contours of wall-pressure disturbance at (a) $t^{*}=0.17 \mathrm{~ms}$, (b) $t^{*}=0.29 \mathrm{~ms}$, (c) $t^{*}=0.41 \mathrm{~ms}$ and (d) $t^{*}=0.52 \mathrm{~ms}$. All the plots have the same contour levels. Isothermal Wall. 

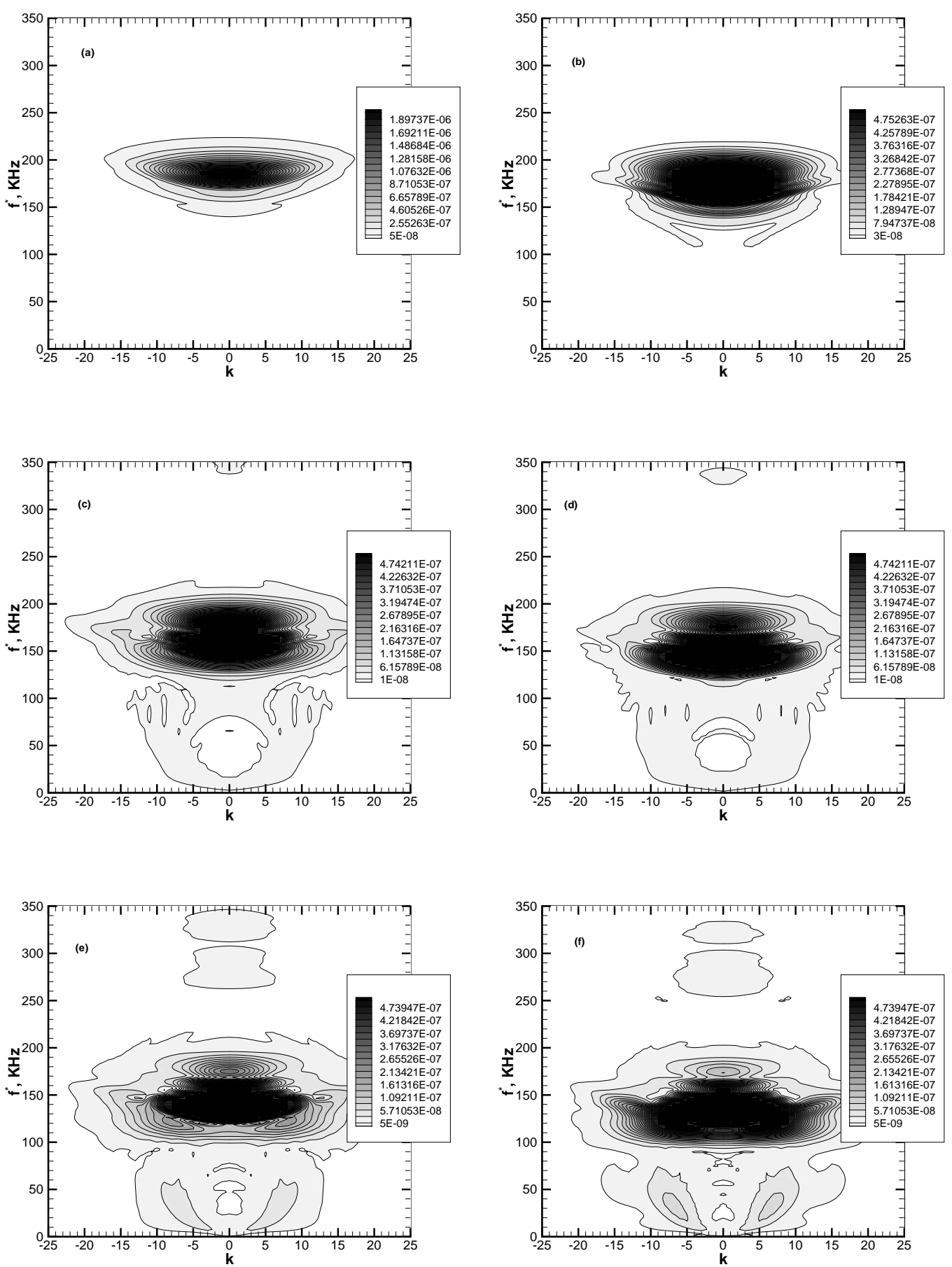

Figure 4.36: Disturbance spectrum in the spanwise mode number - frequency $\left(k-f^{*}\right)$ plane for the simulation with higher forcing amplitude obtained from wall-pressure at different streamwise positions. (a) $x^{*}=0.250 \mathrm{~m}$, (b) $x^{*}=0.300 \mathrm{~m}$, (c) $x^{*}=0.350 \mathrm{~m}$, (d) $x^{*}=0.400 \mathrm{~m}$, (e) $x^{*}=0.450 \mathrm{~m}$ and (f) $x^{*}=0.500 \mathrm{~m}$. 
fundamental resonance is much stronger than subharmonic resonance.

\subsection{Strongly Nonlinear Wave Packet}

Results discussed in the previous section indicate a possible presence of fundamental and subharmonic resonance mechanisms in a weakly nonlinear wave packet. Indications were that fundamental resonance was stronger than subharmonic resonance. In addition to these two resonance mechanisms, the strong peaks observed for lowwave-number second-mode oblique waves indicate the possible presence of an oblique breakdown mechanism. Both the linear and the weakly nonlinear regime were qualitatively similar for the isothermal and adiabatic wall conditions considered. In order to explore which of these mechanisms will prevail in the more strongly nonlinear regime and will lead to nonlinear turbulent breakdown, another simulation with a higher forcing amplitude (5\% of the free-stream velocity) was performed. The forcing amplitude was chosen carefully such that the wave packet would still, at first, grow linearly before reaching the nonlinear regime. Here, only an adiabatic wall condition is considered, since results were qualitatively similar for the two wall boundary conditions considered for the linear and weakly nonlinear wave packets.

\subsubsection{Initial development of the wave packet}

The evolution of the wall-pressure disturbance amplitude at the centerline is shown in figure 4.37. Again as in the previous two sections, in this figure the scale given with the bottom signal indicates the disturbance amplitude. Initially from $t^{*}=0.091 \mathrm{~ms}$ to $t^{*}=0.183 \mathrm{~ms}$ the shape of the wave packet looks similar to that of the linear wave packet. This suggests that the wave packet grows linearly in the beginning. Later at $t^{*}=0.274 \mathrm{~ms}$, the shape of the wave packet changes rapidly and much stronger than for the weakly nonlinear case. The packet appears to be splitting into two parts as it propagates in the downstream direction. Then the waves start to breakdown, 


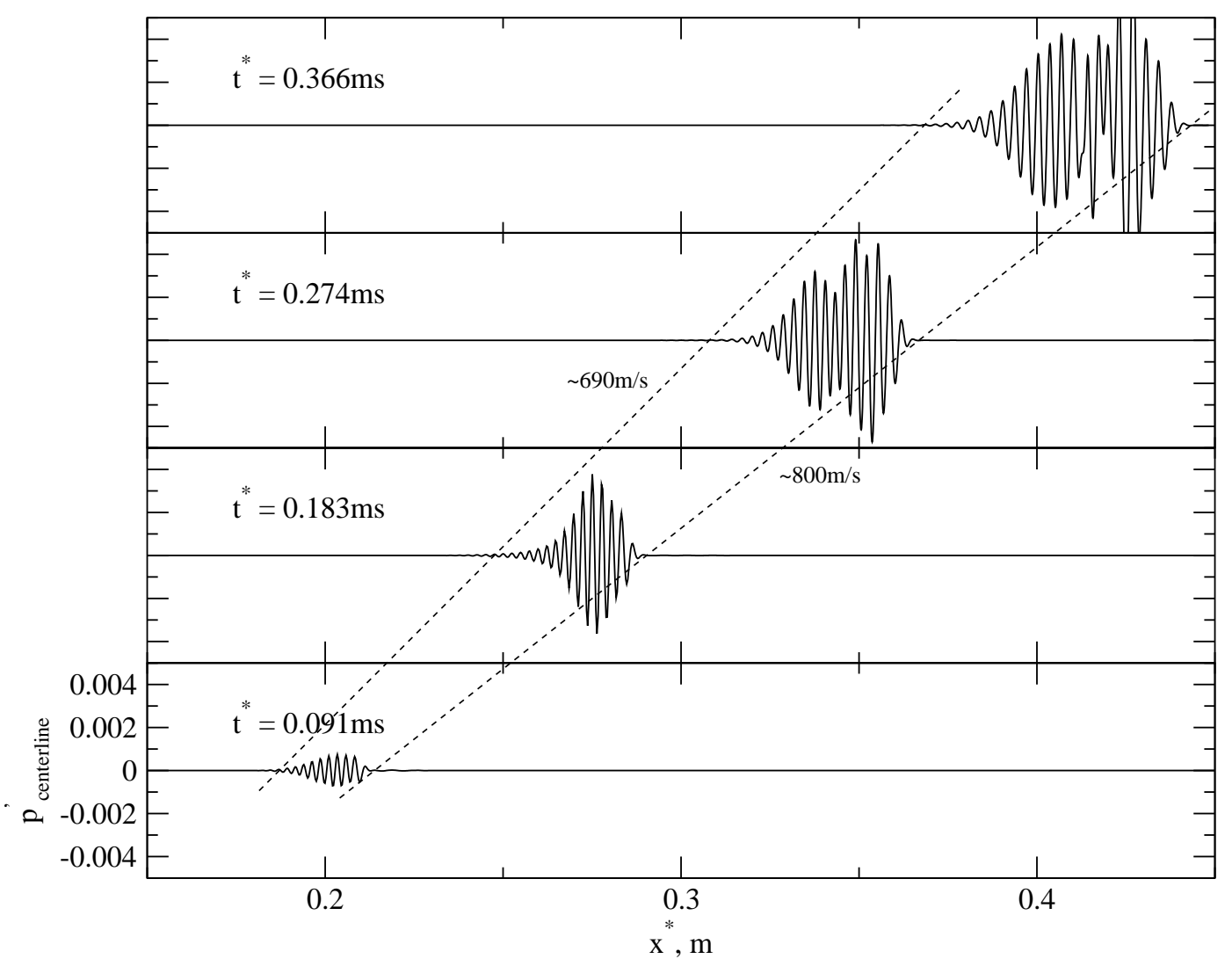

Figure 4.37: Temporal and spatial evolution of wall-pressure disturbance amplitude along the centerline for the strongly nonlinear wave packet. The locations of the front and the tail of the wave packet are indicated by dashed lines. Adiabatic wall. 


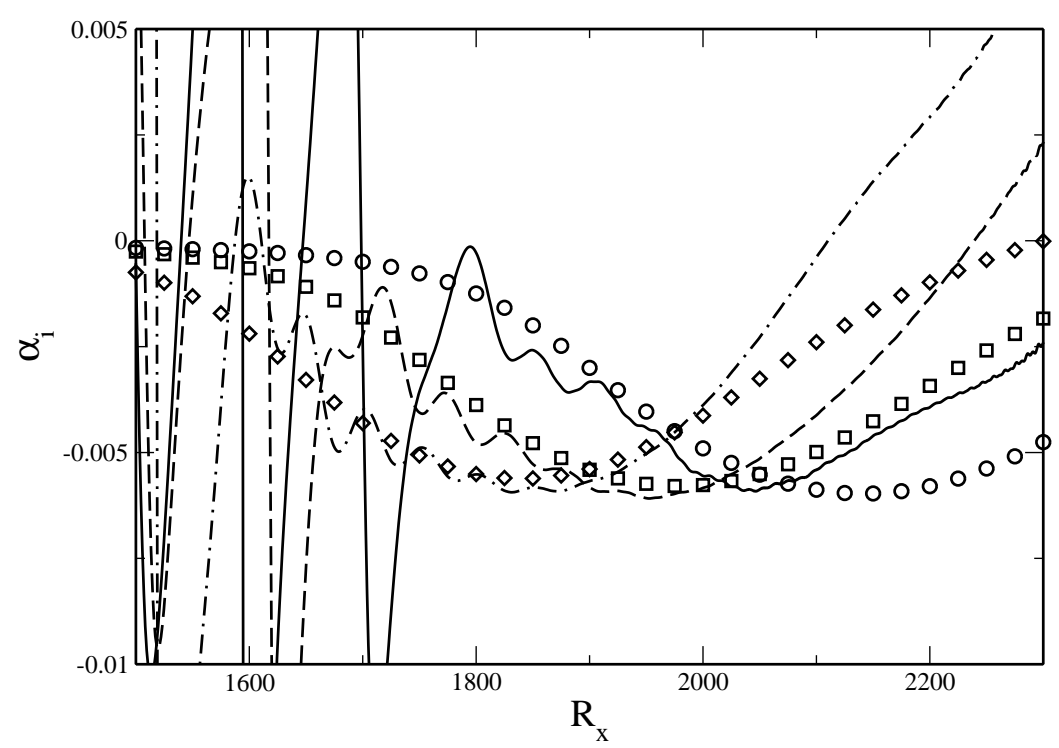

Figure 4.38: Comparison of spatial growth rates $\left(\alpha_{i}\right)$ for three 2D disturbance waves with frequencies (a) $F=1.22 E-04(-, \circ)$ (b) $F=1.32 E-04(---$, $\square)$ and (c) $F=1.42 E-04(-.-.-, \diamond)$. lines are DNS results and symbols are LST results. LST results are computed using Mack's solver (Mack, 1965, 1987). Adiabatic Wall.

first in the middle of the packet with second-mode waves still visible on either side. This observation is in qualitative agreement with wave packet measurements made on the nozzle wall of BAM6QT. There too, breakdown began to appear first in the middle of a nonlinear wave packet (Casper et al., 2011). The location of the front and the tail of the wave packet are indicated by dashed lines. The front end of the wave packet propagates at a velocity of about $800 \mathrm{~m} / \mathrm{s}$ and the tail propagates at a lower velocity of about $690 \mathrm{~m} / \mathrm{s}$. Note that the propagation speeds are only an approximate estimation.

In order to verify whether the initial wave packet indeed grows linearly, in figure 4.38 the spatial streamwise amplification rates are compared $\left(\alpha_{i}\right)$ for several axisymmetric disturbance waves obtained from the DNS with results from LST. The spatial growth rate $\left(\alpha_{i}\right)$ is calculated based on the wall-pressure disturbance amplitude. As observed for the linear wave packets, close to the forcing location the spatial growth rate $\left(\alpha_{i}\right)$ calculated from DNS is modulated by the superposition of 
damped waves. Nevertheless, figure 4.38 confirms that the disturbances within the wave packet grow linearly before reaching the nonlinear regime.

In figure 4.39 snapshots are shown for several time instances, which correspond to those in figure 4.37 and provide a visual impression of the initial evolution for the strongly nonlinear wave packet. The snapshot shown in figure 4.39a for the strongly nonlinear wave packet resembles the linear wave packet shown in figure $4.7 \mathrm{a}$. Weak three-dimensional waves are leading the wave packet in the downstream direction. These could be acoustic waves traveling slightly faster than the main wave packet. Within the wave packet, predominantly two-dimensional or axisymmetric wave fronts exist as in the linear and weakly nonlinear case. In addition, in the snapshot shown in figure $4.39 \mathrm{~b}$ the wave packet still looks similar to the linear wave packet shown in figure $4.7 \mathrm{~b}$, confirming that the wave packet grows linearly at first. However, later at $t^{*}=0.274 m s$ (figure $4.39 \mathrm{c}$ ) the dominant axisymmetric wave fronts are broken up due to nonlinear interactions. Moreover, weak oblique waves develop along the lateral sides of the wave packet. At a later time in figure $4.39 \mathrm{~d}$ for $t^{*}=0.366 \mathrm{~ms}$, the shape of the packet becomes even more deformed. In particular in the center of the wave packet the axisymmetric wave fronts appear to be breaking up into smaller structures. Along the lateral sides of the wave packet the oblique waves appear to become more dominant than before.

In order to analyze the disturbance field, spectra were obtained from the Fourier transformation of the wall-pressure disturbance data using 400 Fourier modes in time. The streamwise development of the disturbance spectrum is shown in figure 4.40. As expected, the spectra in figure 4.40a exhibit a maximum at the azimuthal mode number $k_{c}=0$. Also, the spectra in figure 4.40 a look very similar to those of the linear wave packet, which again confirms that initially the wave packet develops linearly. The spectrum broadens in the downstream direction and in figure 4.40b the spectrum looks slightly deformed, which might be due to weak nonlinear interactions. The disturbance spectrum for $x^{*}=0.28 m$ (figure 4.40c) shows strong indications of nonlinear 

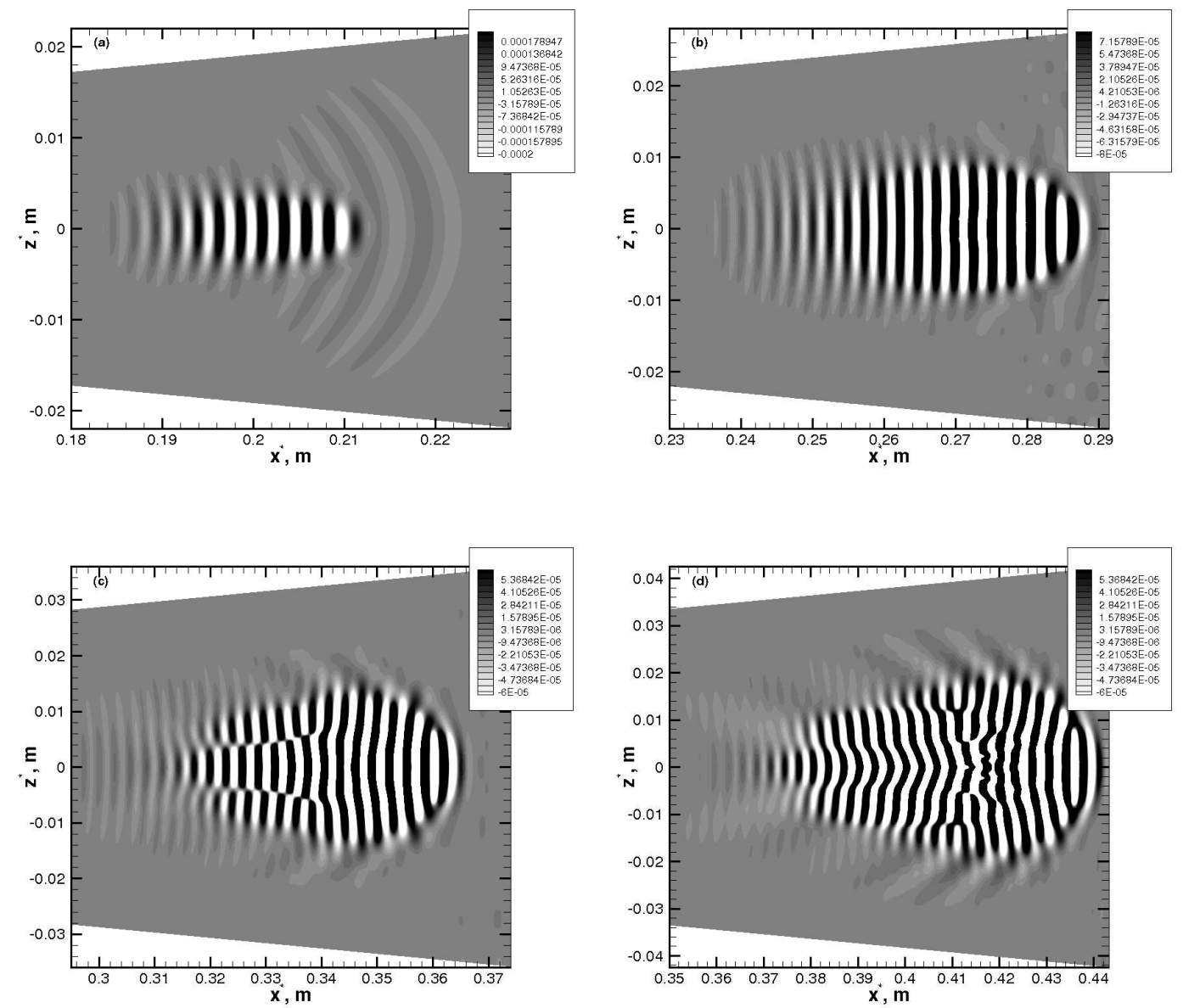

Figure 4.39: Snapshots of the strongly nonlinear wave packet corresponding to the centerline wall-pressure disturbance distributions shown in figure 4.37. Shown are contours of wall-pressure disturbance on the unrolled cone surface at (a) $t^{*}=0.091 \mathrm{~ms}$ (b) $t^{*}=0.183 \mathrm{~ms}(\mathrm{c}) t^{*}=0.274 \mathrm{~ms}$ and $(\mathrm{d}) t^{*}=0.366 \mathrm{~ms}$. Adiabatic Wall. 

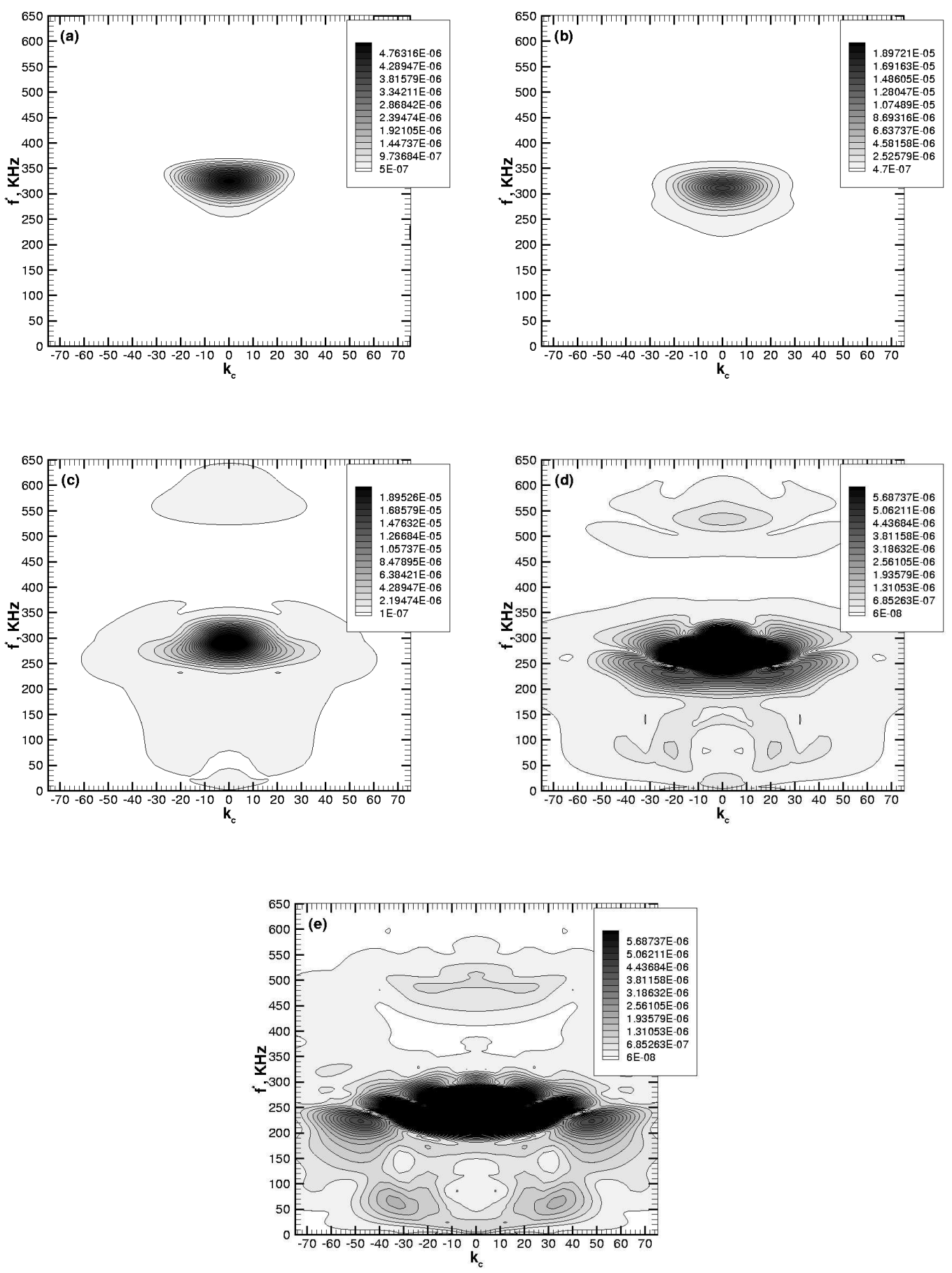

Figure 4.40: Disturbance spectrum in the azimuthal mode number - frequency $\left(k_{c}-\right.$ $\left.f^{*}\right)$ plane obtained from wall-pressure disturbance at various streamwise positions. (a) $x^{*}=0.20 m$, (b) $x^{*}=0.24 m$, (c) $x^{*}=0.28 m$, (d) $x^{*}=0.32 m$ and (e) $x^{*}=0.36 m$. Adiabatic wall. 
interactions as the spectrum broadens with respect to the azimuthal wave number range, and additional frequency bands develop. For $x^{*}=0.32 m$ (figure $4.40 \mathrm{~d}$ ) the spectrum has spread further over higher azimuthal mode numbers, and continues to develop other frequency bands. In addition, low-frequency first-mode oblique waves could also be identified along with other lower frequency waves, which may have been generated nonlinearly. This development intensifies in the downstream direction and the spectrum for $x^{*}=0.36 \mathrm{~m}$ in figure $4.40 \mathrm{e}$ shows strong secondary peaks at the fundamental frequency $f^{*} \approx 250 \mathrm{KHz}$ for larger azimuthal wave numbers. This development may be an indication of a fundamental resonance mechanism. Note that the spectrum in figure 4.40 shows no presence of a subharmonic resonance mechanism. Hence, fundamental resonance might be the dominant nonlinear mechanism for the investigated flow. However, strong peaks could still be observed for low-wavenumber second-mode oblique waves indicating the presence of oblique mechanisms. Both fundamental resonance and oblique breakdown mechanisms may play a role in the natural transition process. Higher harmonics of the high-amplitude frequency bands are also visible in figure $4.40 \mathrm{c}, \mathrm{d}$ and e.

\subsubsection{Flow structures within the turbulent spot}

To gain more insight into the flow structures generated by these strongly nonlinear interactions, iso-surfaces of total vorticity are shown in figures 4.41 and 4.42. Side views and top views of iso-surfaces of total vorticity are shown for $t^{*}=0.366 \mathrm{~ms}$ (see figure 4.41) and $t^{*}=0.4 \mathrm{~ms}$ (see figure 4.42). The iso-surfaces shown here for $t^{*}=0.366 \mathrm{~ms}$, correspond to the wall-pressure disturbance contours of figure $4.39 \mathrm{~d}$. Below the spot (close to the wall) dominant spanwise structures are present during the entire spot formation process. Such spanwise structures were also observed by Jocksch \& Kleiser (2008) in a Mach 5 flat-plate boundary layer and by Krishnan \& Sandham (2006a) in their Mach 6 flat-plate boundary layer simulations. These spanwise- 


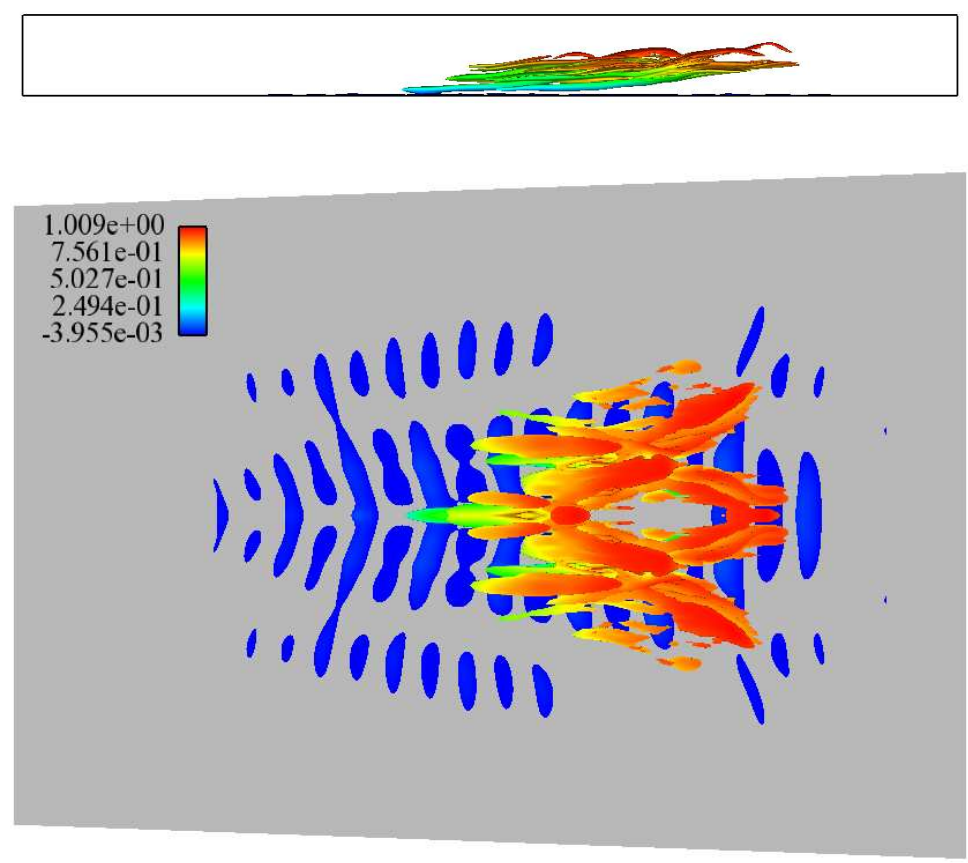

Figure 4.41: Visualization of spot using total vorticity iso-surface. Shown are side view and top view of total vorticity iso-surface at $t^{*}=0.366 \mathrm{~ms}$ on the unrolled cone surface. Iso-surface is coloured with streamwise velocity magnitude. Both plots have the same total vorticity magnitude of 500. Adiabatic Wall. 

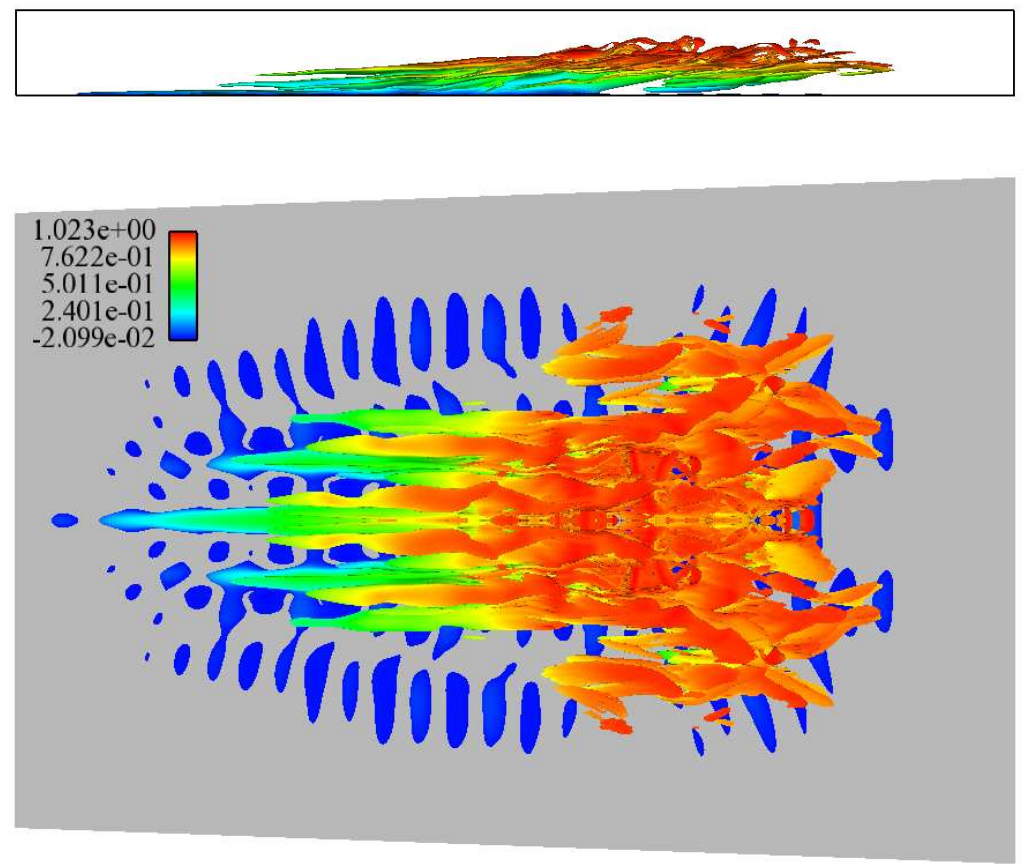

Figure 4.42: Visualization of spot using total vorticity iso-surface. Shown are side view and top view of total vorticity iso-surface at $t^{*}=0.4 \mathrm{~ms}$ on the unrolled cone surface. Iso-surface is coloured with streamwise velocity magnitude. Both plots have the same total vorticity magnitude of 500. Adiabatic wall. 
oriented structures are larger and more ordered compared to the structures away from the wall and they spread all along the wall from the leading edge of the developing spot to the tail. These spanwise structures seen in the hypersonic boundary layers are not seen in supersonic (first mode dominant) boundary layers (Jocksch \& Kleiser, 2008). In hypersonic boundary layers additional inviscid modes are present and the instability mechanism involves reflection of acoustic waves between the solid surface and the sonic line. Therefore, the location and shape of these spanwise structures suggests that these might be related to (second mode waves) Mack modes (Krishnan \& Sandham, 2006a).

Later at $t^{*}=0.4 \mathrm{~ms}$ (see figure 4.42), these structures develop into a localized turbulent region or turbulent spot. The localized turbulent region starts to show typical properties of a turbulent spot. In the tail region streamwise elongated structures can be identified (can be seen in the side view and the top view). In the top view, small-scale structures can be seen to develop in the center of the spot, while the spanwise structures are still dominant starting from the leading edge of the spot to the center. However, the strength of these spanwise structures decreases towards the tail of the spot. The fact that the region from the tip to the center of the spot is the region where the local transition process takes place, suggests the occurrence of these spanwise structures as a transitional phenomenon (Jocksch \& Kleiser, 2008).

For $t^{*}=0.423 m s$ in figure 4.43 the turbulent spot is even more developed, showing even clearer characteristics of a "young" turbulent spot. In the end view (figure 4.43a), the "wingtip" with the typical lateral overhang can be clearly identified. In figure $4.43 \mathrm{~b}$ a typical overhang region at the leading edge of the turbulent spot can be observed. Hairpin-shaped substructures, which play an important role in turbulence production mechanisms, are clearly visible in the top view (see figure 4.43c). A further developed mature turbulent spot at $t^{*}=0.490 \mathrm{~ms}$ is shown in figure 4.44 . A top view (figure $4.44 \mathrm{c}$ ) of the spot shows the arrowhead-shape of the core. In addition, streamwise elongated structures could be seen extending from the center 


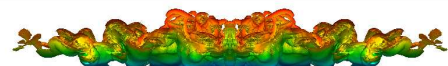

(a) End view

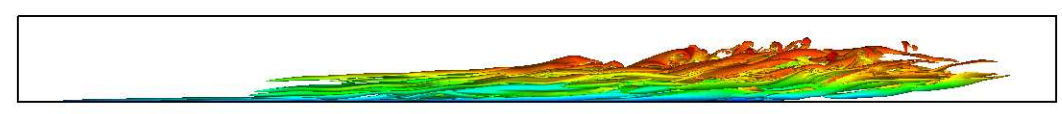

(b) Side view

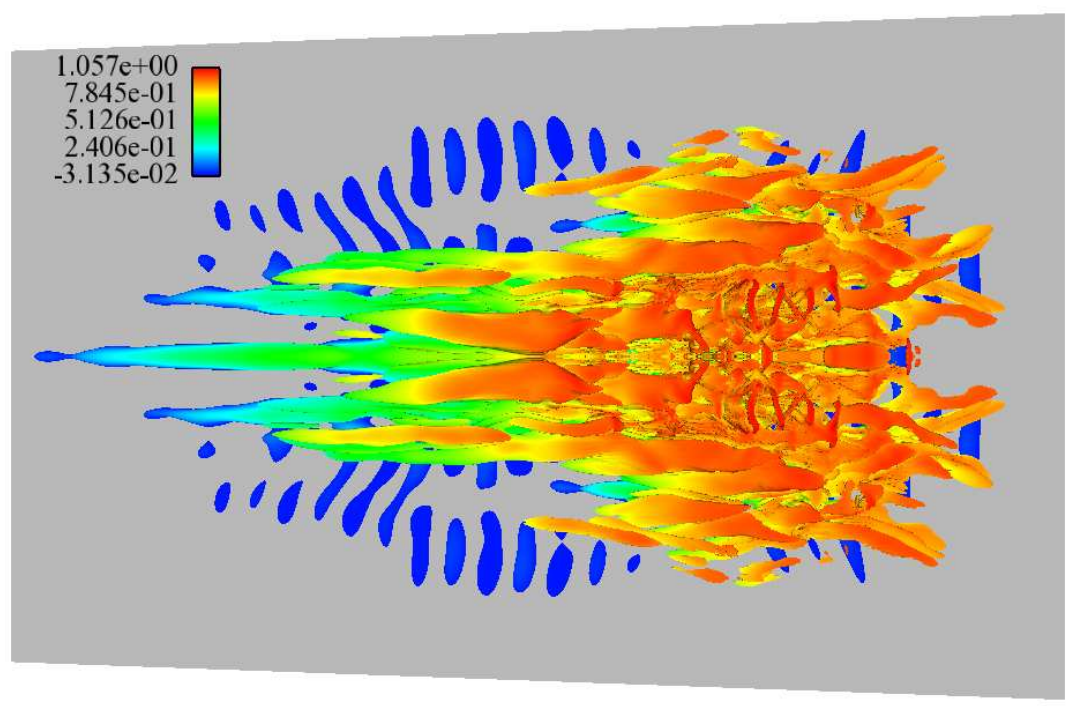

(c) Top view

Figure 4.43: Visualisation of a "young" turbulent spot at $t^{*}=0.423 \mathrm{~ms}$. Shown are (a) end view, (b) side view and (c) top view of total vorticity iso-surfaces on the unrolled cone surface. Iso-surfaces are coloured with streamwise velocity magnitude. All the plots have the same total vorticity magnitude of 600 . Adiabatic wall. 
(a) End view

(b) Side view

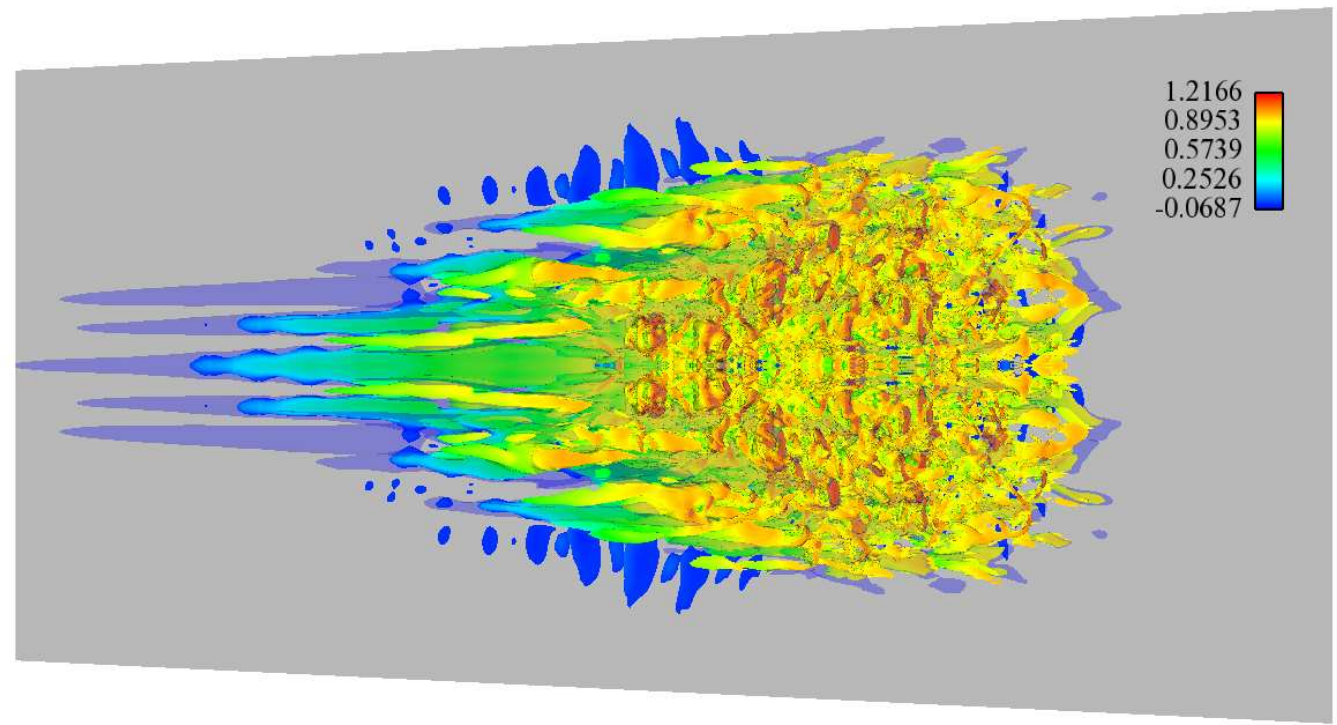

(c) Top view

Figure 4.44: Visualisation of a "mature" turbulent spot at $t^{*}=0.490 \mathrm{~ms}$. Shown are (a) end view, (b) side view and (c) top view of total vorticity iso-surfaces on the unrolled cone surface. Iso-surfaces are coloured with streamwise velocity magnitude. All the plots have the same total vorticity magnitude of 700 . Adiabatic Wall. 


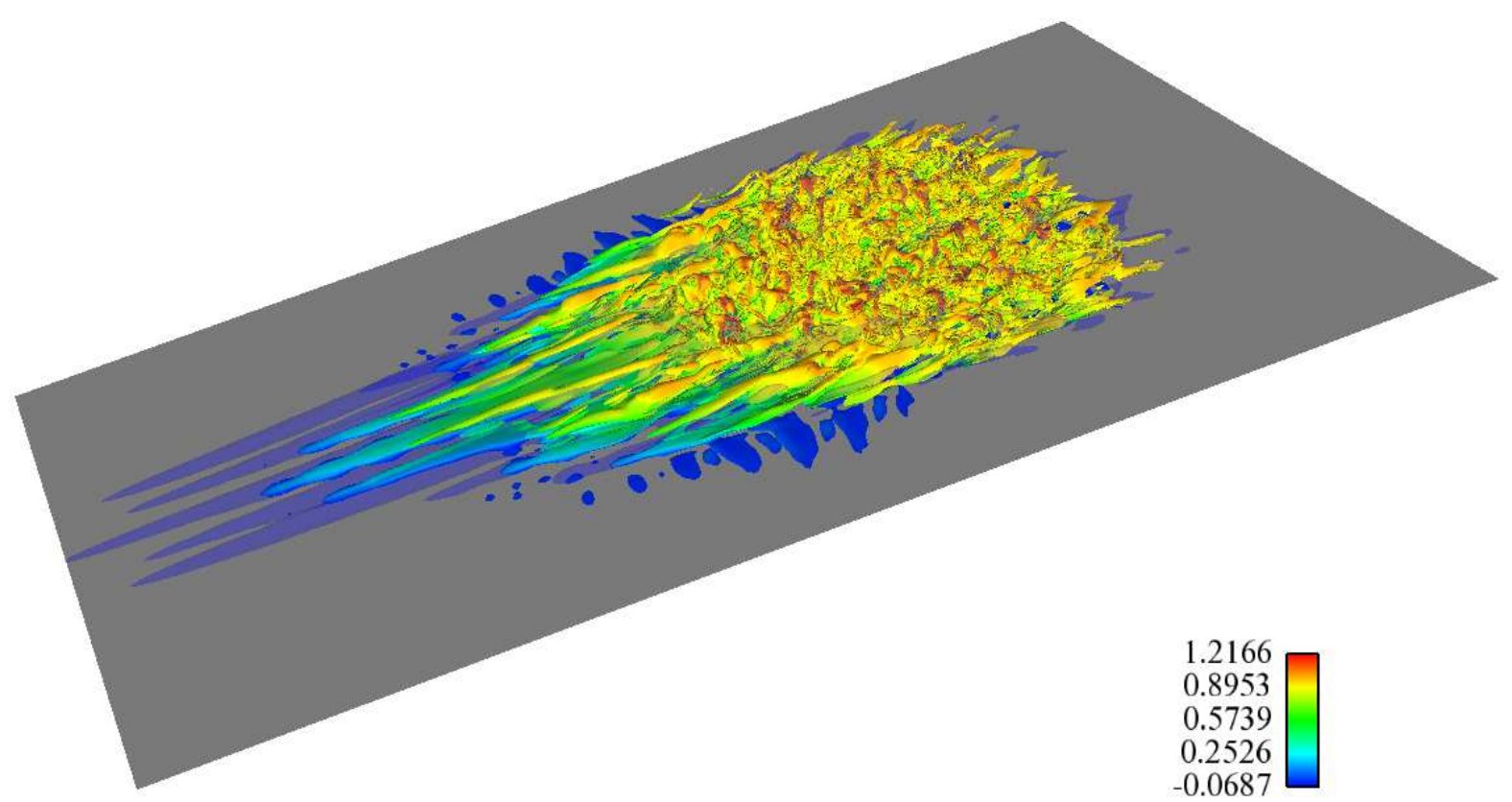

(a) Perspective view

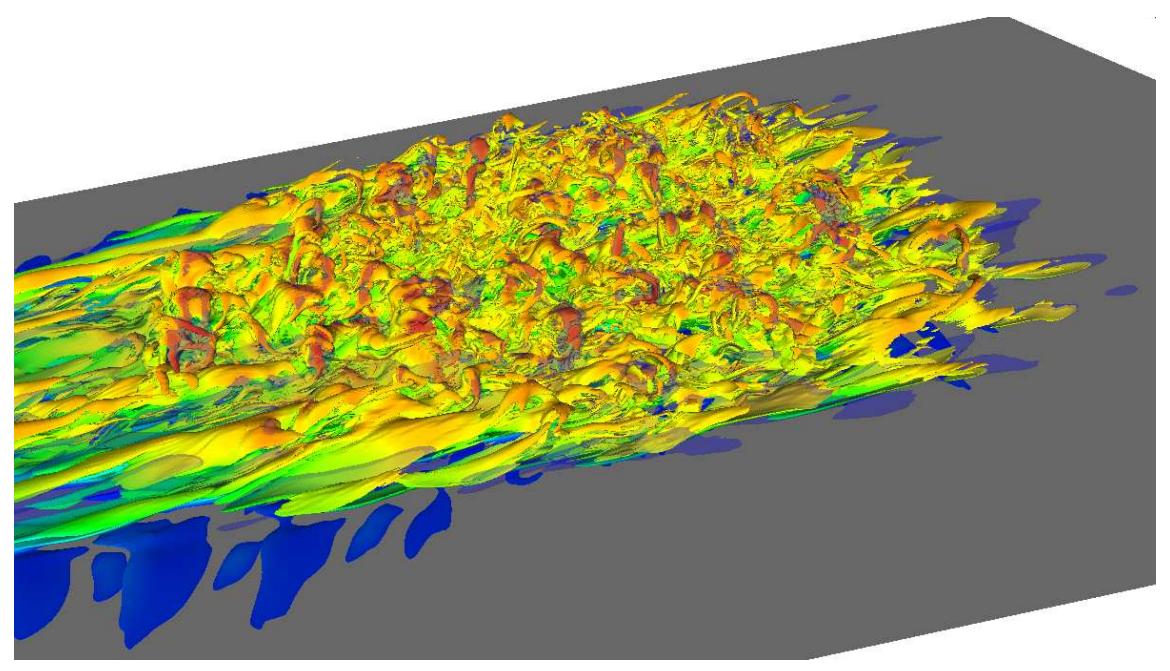

(b) Close-up of the turbulent core

Figure 4.45: (a) Perspective view of the turbulent spot and (b) Close-up view of the turbulent core region of the spot. Shown are iso-surface of total vorticity on the unrolled cone surface at $t^{*}=0.490 \mathrm{~ms}$. Iso-surfaces are coloured with streamwise velocity magnitude. Both plots have the same total vorticity magnitude of 700 . Adiabatic Wall. 
region of the spot to the tail region. Structures at the tail region of the spot are very close to the wall (see side view in figure 4.44b). This region is the so-called calm area behind the turbulent spot. A perspective view of the turbulent spot is shown in figure $4.45 \mathrm{a}$ and a close- $\mathrm{up}$ view of the turbulent core region of the spot is shown in figure $4.45 \mathrm{~b}$.

To gain more information about substructures within the turbulent spot, contours of streamwise vorticity are shown in figure 4.46 in $y-\varphi$ planes at five selected streamwise (constant $x$ ) locations along the "young" turbulent spot shown in figure 4.43. Dark contour regions represent positive streamwise vorticity and white regions represent negative streamwise vorticity. The interesting features seen in each of the planes are discussed as follows. The first plane at $x^{*}=0.437 m$ (figure $4.46 \mathrm{a}$ ) is in the tail/calm region of the young turbulent spot where shear exists at the wall but no other large-scale flow structures are present. In figure $4.46 \mathrm{~b}$, the streamwise vorticity on the $y-\varphi$ plane at $x^{*}=0.445 m$ is shown. Here stronger shear exists close to the wall and in addition to that, significant streamwise vorticity can be seen for $y<0.0016 \mathrm{~m}$ and $|\varphi|<0.1 \mathrm{rad}$. Note that the near-wall activity is concentrated in the central regions of the spot and near the spanwise edge of the spot, the regions of concentrated vorticity exist farther away from the wall. The near-wall streamwise vorticity concentrations become even more confined to the central region of the turbulent spot farther downstream at $x^{*}=0.453 \mathrm{~m}$ (see figure 4.46c). The streamwise vorticity contour shown in $y-\varphi$ plane for $x^{*}=0.461 \mathrm{~m}$ (figure $4.46 \mathrm{~d}$ ) is in the turbulent core region of the spot. Here, almost all of the near-wall vorticity concentrations are confined to $|\varphi|<0.15 \mathrm{rad}$ and away from the wall strong vorticity concentration exists for $|\varphi|<0.25 \mathrm{rad}$. At $x^{*}=0.469 \mathrm{~m}$ (figure 4.46e), there is almost no near-wall activity. This location is in the leading edge overhang region. Figure 4.46e suggests that the overhang region of the spot consists of vortices that do not contribute to the generation of near-wall turbulence.

Another set of streamwise vorticity contours is plotted in figure 4.47 for a later time 
$t^{*}=0.490 \mathrm{~ms}$. Here, the selected streamwise positions for the "mature" turbulent spot are similar to those of the "young" spot in figure 4.46. Similar observations, as for the "young" spot could be made for the "mature" spot in figure 4.47. However, there is a higher concentration of streamwise vorticity at the corresponding locations in the case of the "mature" spot. In addition, the concentration of streamwise vorticity has spread farther in both the wall-normal and spanwise directions. The contour in $y-\varphi$ plane for $x^{*}=0.510 m$ (figure $4.47 \mathrm{~d}$ ) shows strong vorticity concetration for $y<0.004 m$ and $|\varphi|<0.35 \mathrm{rad}$.

Figure 4.48 shows spanwise vorticity on the $x-y$ plane for various azimuthal positions. The five selected azimuthal positions are (a) $\varphi=0$, (b) $\varphi=0.048$, (c) $\varphi=0.077$, (d) $\varphi=0.097$ and (e) $\varphi=0.117$. Here dark contour regions represent negative spanwise vorticity and white regions represent positive spanwise vorticity. At all azimuthal positions weak concentrations of spanwise vorticity that are farthest downstream and away from the wall coincide with the leading edge overhang region. Upstream of this location, strong spanwise vorticity can be identified high in the boundary layer: this corresponds to the turbulent core region. These turbulent structures (seen clearly in figure 4.48b) are the heads of hair-pin-like vortices that are slowly rolling up. Farther upstream, several inclined vorticity layers could be identified close to the wall and within the boundary layer. These vorticity layers appear to have their base in the near-wall regions. In figure 4.49, spanwise vorticity contours are shown for the same azimuthal positions at a later time $t^{*}=0.490 \mathrm{~ms}$. At all azimuthal positions, structures similar to those observed at $t^{*}=0.423 \mathrm{~ms}$ (figure 4.48) can still be identified. However, a much stronger concentration of spanwise vorticity exists high in the boundary layer at all azimuthal positions in the turbulent core region of the spot.

Contours of wall-normal density gradient are shown in figure 4.50 for several azimuthal positions. The selected azimuthal positions are the same as the ones in figure 4.48. As for spanwise vorticity, wall-normal density gradient contours are also 

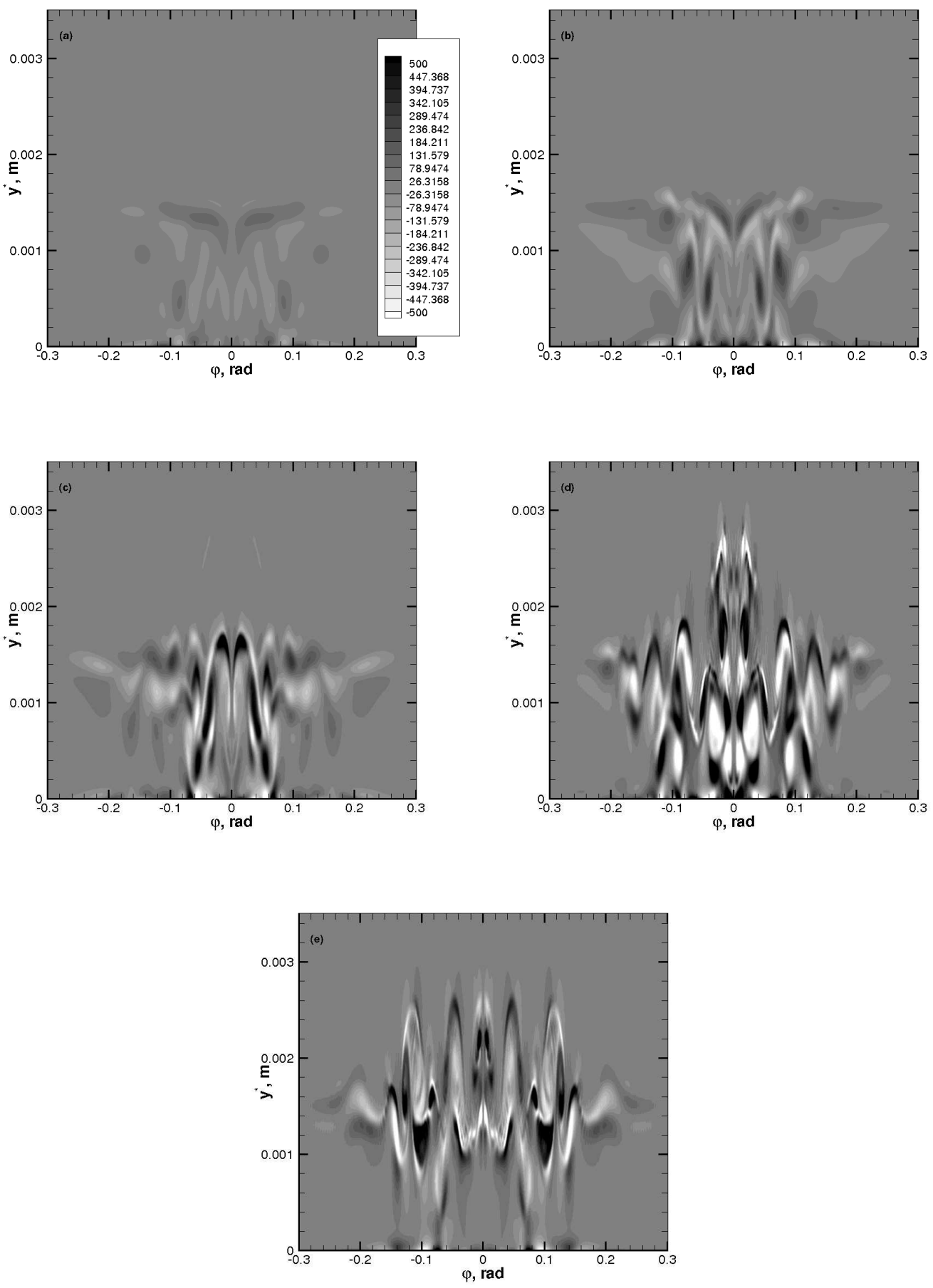

Figure 4.46: Contours of streamwise vorticity in $y-\varphi$ plane obtained for five streamwise locations at $t^{*}=0.423 \mathrm{~ms}$. (a) $x^{*}=0.437 \mathrm{~m}$, (b) $x^{*}=0.445 \mathrm{~m}$, (c) $x^{*}=0.453 \mathrm{~m}$, (d) $x^{*}=0.461 \mathrm{~m}$ and (e) $x^{*}=0.469 \mathrm{~m}$. All the plots have the same contour levels. Adiabatic wall. 

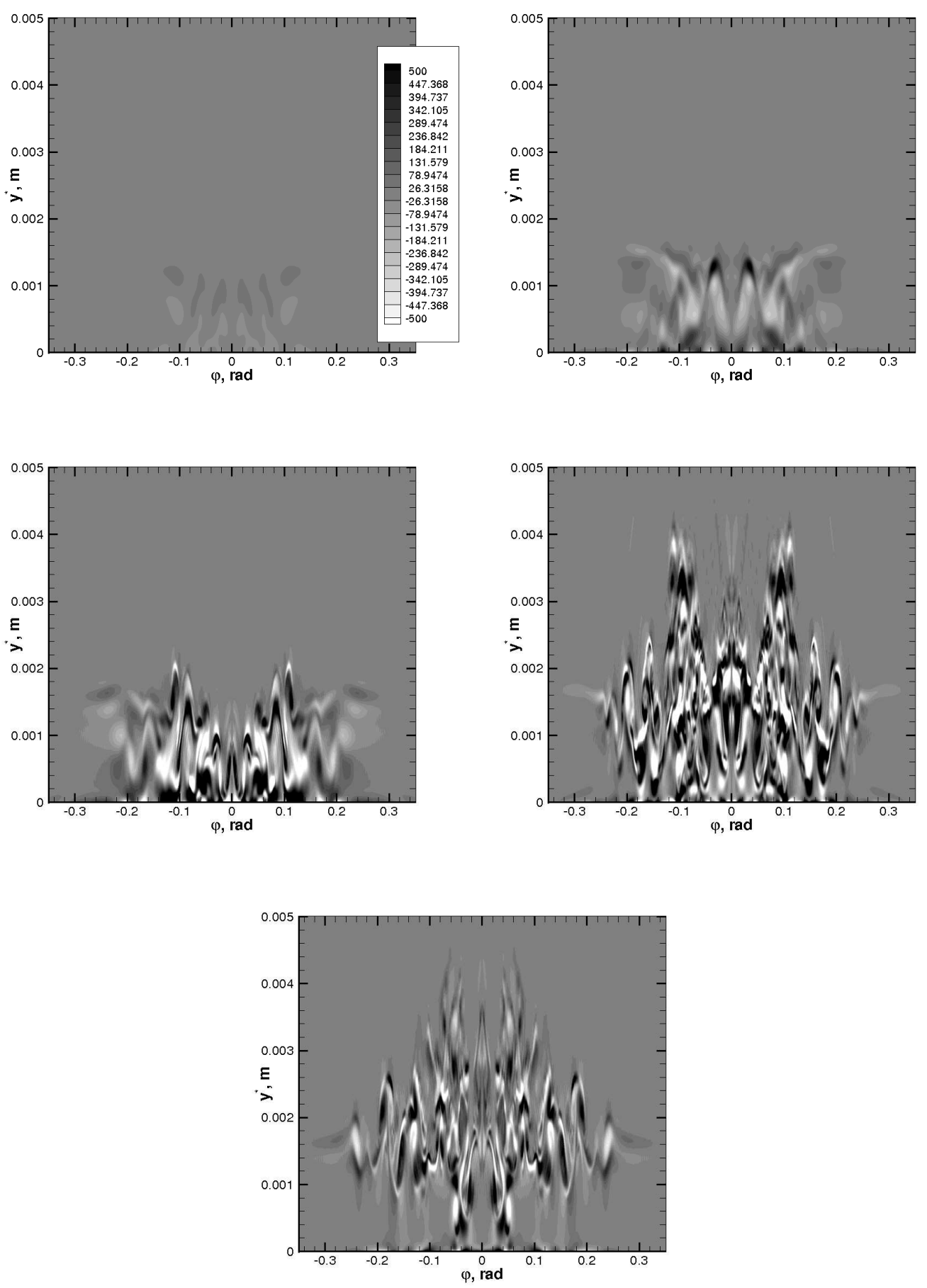

Figure 4.47: Contours of streamwise vorticity in $y-\varphi$ plane obtained for five streamwise locations at $t^{*}=0.490 \mathrm{~ms}$. (a) $x^{*}=0.469 \mathrm{~m}$, (b) $x^{*}=0.486 \mathrm{~m}$, (c) $x^{*}=0.496 \mathrm{~m}$, (d) $x^{*}=0.510 \mathrm{~m}$ and (e) $x^{*}=0.526 \mathrm{~m}$. All the plots have the same contour levels. Adiabatic wall. 

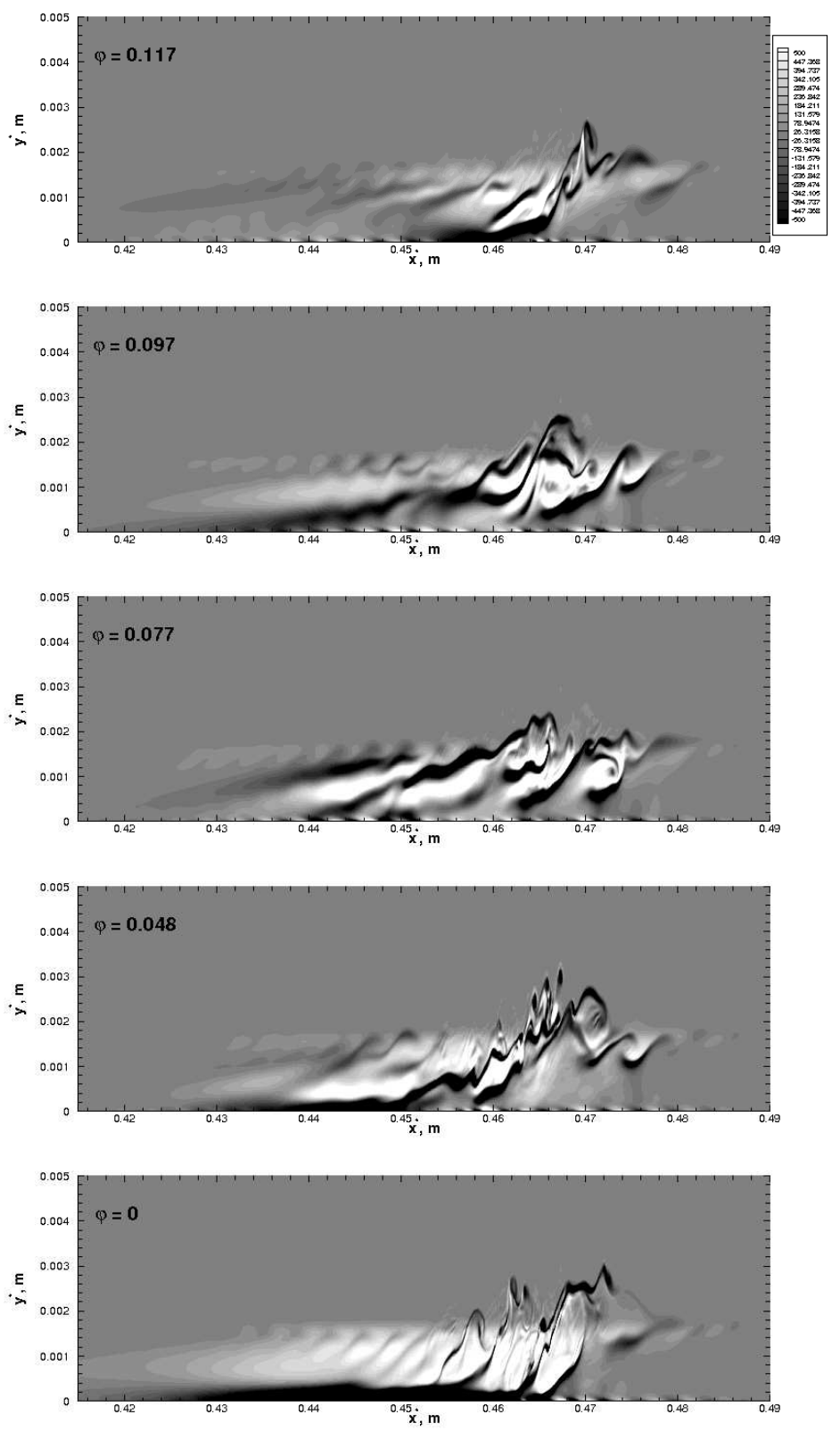

Figure 4.48: Contours of spanwise vorticity on the $x-y$ plane for five azimuthal positions at $t^{*}=0.423 \mathrm{~ms}$. (a) $\varphi=0$, (b) $\varphi=0.048$, (c) $\varphi=0.077$, (d) $\varphi=0.097$ and (e) $\varphi=0.117$ (from bottom to top). All the plots have the same contour levels. Adiabatic Wall. 

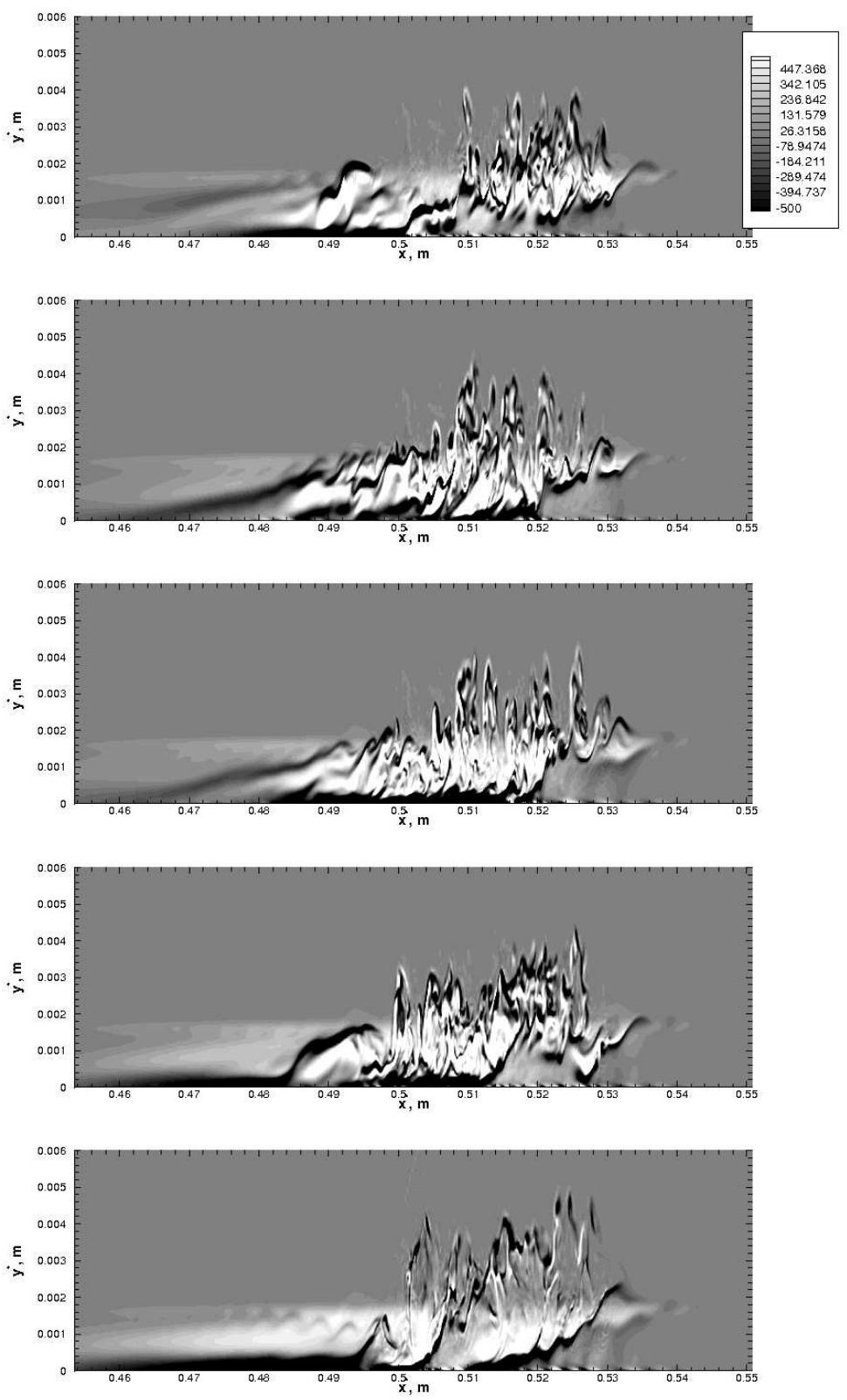

Figure 4.49: Contours of spanwise vorticity on the $x-y$ plane for five azimuthal positions at $t^{*}=0.490 \mathrm{~ms}$. (a) $\varphi=0$, (b) $\varphi=0.048$, (c) $\varphi=0.077$, (d) $\varphi=0.097$ and (e) $\varphi=0.117$ (from bottom to top). All the plots have the same contour levels. Adiabatic Wall. 

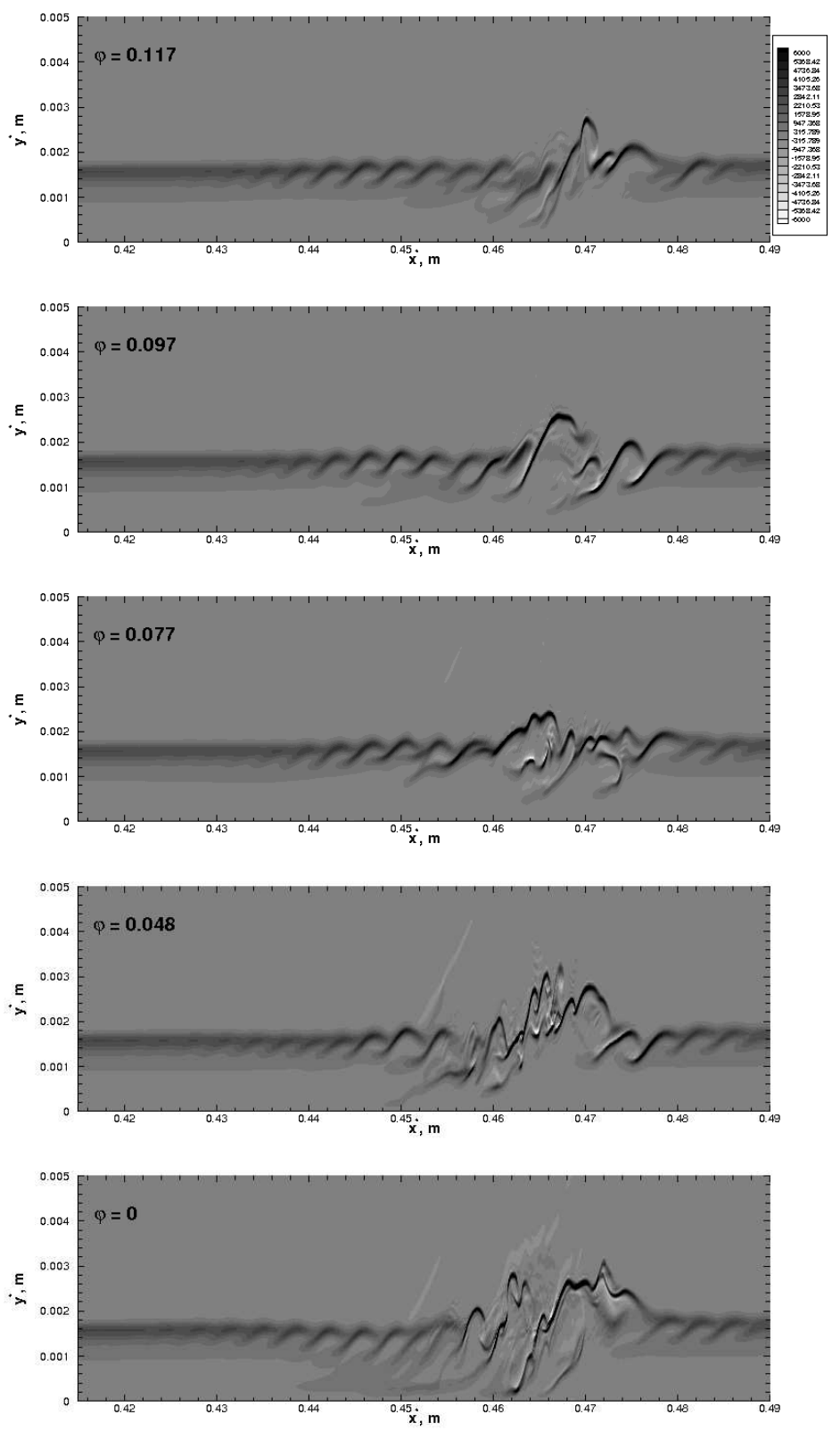

Figure 4.50: Contours of wall-normal density gradient on the $x-y$ plane for five azimuthal positions at $t^{*}=0.423 \mathrm{~ms}$. (a) $\varphi=0$, (b) $\varphi=0.048$, (c) $\varphi=0.077$, (d) $\varphi=0.097$ and (e) $\varphi=0.117$ (from bottom to top). All the plots have the same contour levels. Adiabatic Wall. 

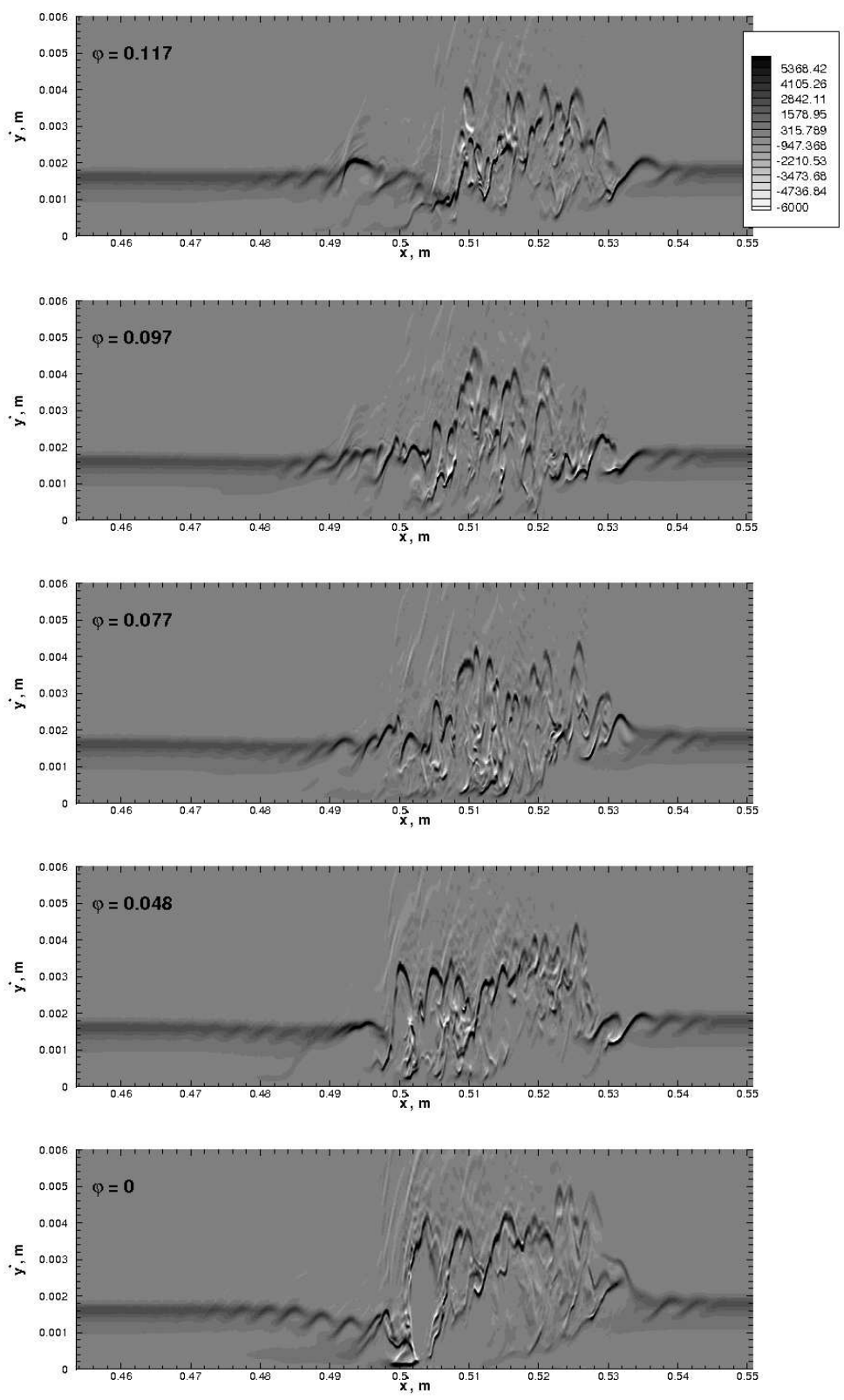

Figure 4.51: Contours of wall-normal density gradient on the $x-y$ plane for five azimuthal positions at $t^{*}=0.490 \mathrm{~ms}$. (a) $\varphi=0$, (b) $\varphi=0.048$, (c) $\varphi=0.077$, (d) $\varphi=0.097$ and (e) $\varphi=0.117$ (from bottom to top). All the plots have the same contour levels. Adiabatic Wall. 
shown in the $x-y$ plane for each azimuthal positions. This type of flow visualization could be used for comparison with Schlieren pictures taken from experiments. Schlieren photographs are widely utilized to reveal flow structures in high-speed experiments. At all azimuthal positions remarkable "rope-like" structures appear close to the boundary layer edge, both in front and at the tail region of the turbulent spot. Contours of wall-normal density gradient have also been plotted for a later time $t^{*}=0.490 \mathrm{~ms}$ in figure 4.51 for the same five azimuthal positions. The "rope-like" structures are still visible both at the front and the tail region of the spot. These rope-like waves appear to be a phenomenon of high-speed flow. Smith (1994) wrote an excellent review of the literature in regard to these waves. Similar waves were observed in the flow visualization of numerical results for an axisymmetric high-speed boundary layer by Pruett \& Zang (1992). They incorrectly concluded that these were artifacts of secondary instability associated with second-mode primary instability waves. Later, Pruett \& Chang (1995) concurred with Stetson \& Kimmel (1993) in that the original interpretation of these waves was in error and showed these waves to be an artifact of large-amplitude second-mode disturbances. Though the effect of the disturbance obliqueness angle on the appearance of the "ropes" remains unclear, Pruett \& Chang (1995) speculate that equal and opposite oblique second-mode waves will appear rope-like in schlieren images. Note that at all azimuthal locations in addition to the rope-like waves, the wall-normal density gradient contours (figure 4.50 and 4.51) also show the dominant structures near the turbulent core region as seen in the spanwise vorticity contours in figure 4.48 and 4.49 .

\subsubsection{General features of the turbulent spot}

The local spanwise-averaged skin friction coefficient at $t^{*}=0.490 \mathrm{~ms}$ is plotted in figure 4.52 as a function of streamwise distance. Here, the skin friction coefficient is averaged over $|\varphi|<0.148 \mathrm{rad}$. Both the laminar and the turbulent values of the skin 


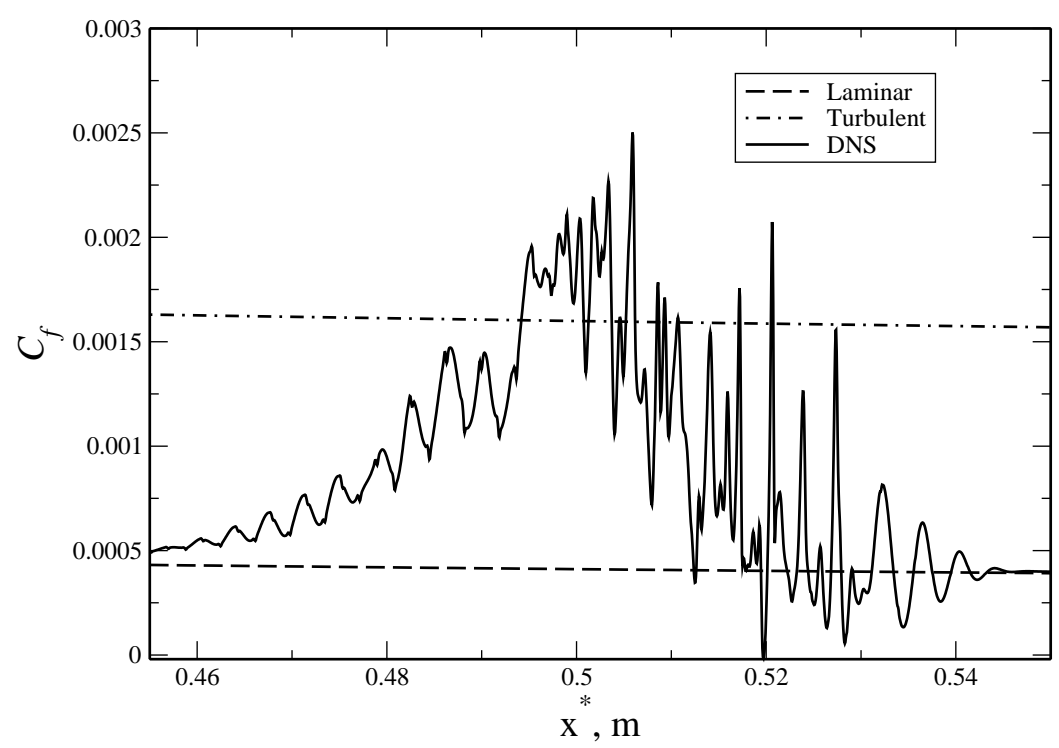

Figure 4.52: Skin friction coefficient as function of streamwise distance at $t^{*}=$ $0.490 \mathrm{~ms}$. Data was averaged in spanwise direction over $|\varphi|<0.148 \mathrm{rad}$. Laminar and turbulent skin-friction coefficients are shown for comparison. Adiabatic Wall.

friction coefficients are shown for comparison. Although the value of skin friction from our DNS modulates greatly, its average over the core region $(0.49 \leq x \leq 0.51)$ of the spot is close to the turbulent value. The largest value of skin friction occur near the center of the spot. Both upstream and downstream to this location, the skin friction decays gradually. A semi-log plot of the spanwise-averaged streamwise velocity profiles at several streamwise locations along the spot core $(0.49 \leq x \leq$ 0.51 ) at $t^{*}=0.490 \mathrm{~ms}$ is shown in figure 4.53a. And a mean of these spanwiseaveraged profiles over the streamwise extent of the spot core is shown in figure 4.53b. The equation for the turbulent viscous sublayer $\left(u^{+}=y^{+}\right)$and the log layer $\left(u^{+}=\right.$ 2.5log $\left.\left(y^{+}\right)+5.0\right)$ are also plotted in figure 4.53. A clear log layer does not exist yet; however, these profiles confirm that a turbulent region is beginning to develop inside the spot.

The physical mechanisms that lead to the growth of a spot in the lateral and wall-normal direction are different. The wall-normal growth of the spot is similar 


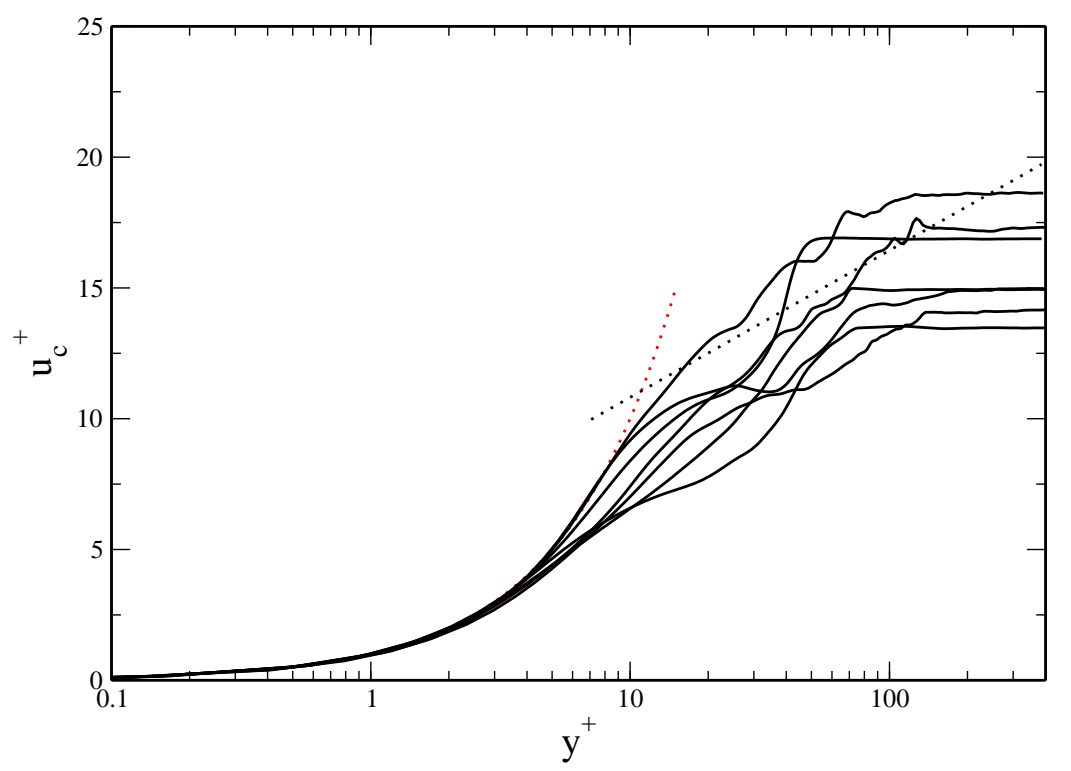

Figure 4.53: Van Driest transformed streamwise velocity profiles normalized with the friction velocity in the spot core at $t^{*}=0.490 \mathrm{~ms}$. Spanwise averaged profiles over $|\varphi|<0.148 \mathrm{rad}$ at several streamwise locations within the spot core $(0.49 \leq x \leq 0.51)$. The dotted line in red indicate the linear sublayer and the dotted line in black indicate the law of the wall. Adiabatic Wall.

to the classical entrainment mechanism. Whereas in the streamwise direction, the difference between the convective speeds of the front and the tail of the spot decides its growth. In the lateral direction the spot is believed to grow by destabilizing the surrounding laminar flow (Gad-el Hak et al., 1981). Fischer (1972) reported the variation of the wall-normal and lateral spreading angles for the disturbance region with local Mach number. The spreading angle relative to the wall remained constant with the Mach number, whereas the lateral spreading angle decreased significantly with the Mach number. Lateral spreading angles of 10 to 11 degrees have been reported for incompressible/subsonic boundary layers. In hypersonic boundary layers lateral spreading angles have been reported in the range from 1.7 to 3 degrees in the literature. For the turbulent spot discussed in this proposal, the lateral spreading angle is 2.3 degrees. 


\subsection{Summary of Wave Packet Simulations}

In this chapter, the growth and breakdown of a wave packet into a turbulent spot was studied for a sharp circular cone at Mach 6. The objective of this research work is to investigate transition initiated by a broad disturbance spectrum as a model of a "natural" transition scenario. In order to generate a wide range of disturbance waves, the boundary layer is forced by a short duration (localized) blowing and suction pulse. The pulsed forcing leads to three-dimensional wave packets, which travel in the downstream direction.

First, the linear development of the wave packet was studied by pulsing the flow with a low amplitude ( $0.001 \%$ of the free-stream velocity). Contours of instantaneous wall-pressure illustrate that near the disturbance source the wave packet has an entirely three-dimensional structure. However, as it propagates downstream, stronger two-dimensional or axisymmetric wave fronts develop. In addition to these axisymmetric wave fronts, oblique waves were also observed on the lateral sides of the wave packet. However, the amplitudes of these oblique waves were small compared to the dominant axisymmetric waves. The wall-pressure disturbance spectrum exhibited a maximum at the azimuthal mode number $k_{c}=0$. The spectrum broadened in the downstream direction as the lower frequency first-mode oblique waves are amplified. However, the peak amplitude remained at $k_{c}=0$ which shifted to lower frequencies in the downstream direction as the boundary layer got thicker. This was expected as for high Mach numbers, two-dimensional or axisymmetric waves experience the highest streamwise amplification rate. Overall, the streamwise development of the disturbances was qualitatively similar for both isothermal and adiabatic wall cases.

To investigate the weakly nonlinear transition regime, the flow was pulsed with a medium-amplitude disturbance ( $0.5 \%$ of the free-stream velocity). Snapshots of instantaneous wall-pressure disturbance contours illustrate that the wave packet develops linearly at first before the high-amplitude axisymmetric wave fronts in the 
wave packet become modulated in the azimuthal direction due to nonlinear interactions. The wall pressure disturbance spectrum confirmed that the wave packets develop linearly at first. However, in the weakly nonlinear regime strong secondary peaks were observed at the fundamental frequency $\left(f^{*} \approx 200 \mathrm{KHz}\right)$ for larger azimuthal wave numbers. This development may be an indication of a fundamental resonance mechanism. In addition, secondary peaks are observed at approximately half the frequency of the high-amplitude frequency band for higher azimuthal mode numbers, which would be an indication of a subharmonic resonance. However, the spectrum suggests that fundamental resonance is much stronger than subharmonic resonance. Strong peaks were also observed for low-wave-number (shallow wave angle) second-mode oblique waves, which indicates a possible presence of a oblique breakdown mechanism. Higher harmonics of the high amplitude frequency band are also visible. Again, the streamwise development of the disturbances was qualitatively similar for the adiabatic and isothermal wall cases.

Finally, to identify more clearly which of these mechanisms are dominant in a strongly nonlinear wave packet and, in particular, to explore which mechanisms ultimately lead to nonlinear turbulent breakdown, a simulation with a higher forcing amplitude (5\% of free-stream velocity) was performed. Contours of instantaneous wall-pressure indicates that the nonlinear wave packet still grows linearly at first before reaching the weakly nonlinear regime. Eventually, farther downstream, strong nonlinear interactions lead to breakdown into local patches of turbulent flow (turbulent spot) surrounded by otherwise laminar flow. Dominant spanwise structures were observed close to the wall during the entire spot formation process. These spanwiseoriented structures are more coherent than the structures away from the wall. Later, these early structures develop into a "mature" turbulent spot. The wall pressure disturbance spectrum indicates that both second-mode fundamental resonance and oblique breakdown mechanisms may be the dominant mechanisms for the investigated flow. Both mechanisms may play a role in the natural transition process for a cone 
boundary layer at Mach 6 . 


\section{CONTROLLED TRANSITION SIMULATIONS}

The wave packet simulations discussed in Chapter 4 have provided strong evidence for a possible presence of fundamental and subharmonic resonance mechanisms in the nonlinear transition regime of a hypersonic cone boundary layer at Mach 6. There were indications that fundamental resonance was much stronger than the subharmonic resonance. In addition to the two resonance mechanisms, the wave packet simulations have also indicated a possible presence of an oblique breakdown mechanism. Both second-mode fundamental resonance and oblique breakdown mechanisms seem to be the relevant nonlinear mechanisms for the investigated flow. However, a nonlinear mechanism is only relevant for the transition process if that mechanism can indeed completely transition a laminar boundary layer to turbulence. Hence, this chapter addresses the important question: Does these nonlinear mechanisms lead to a turbulent boundary layer?

In the wave packet simulations, due to the broadband forcing of multiple frequencies and wave numbers, the transition physics are highly complex. This is particularly true for possible nonlinear interactions between different wave components of the broad spectrum. Therefore, in order to answer the question raised above, it is necessary to perform simulations where the number of possible nonlinear interactions are limited. Towards that end, controlled transition simulations are performed where only a few specified modes are forced. For example, to explore the role of the "classical" fundamental breakdown (K-type), only two modes are forced, i.e.. a primary axisymmetric wave with high amplitude and a pair of oblique waves with small amplitude. In particular, the parameter space is scanned with small and medium scale simulations to identify the "most dangerous" cases for these nonlinear mechanisms. Based on the results of this parameter study, large scale highly resolved simulations that proceed deep into transition to turbulence have been performed and discussed 
in detail in this chapter. Note that in this chapter only an isothermal wall condition is considered, as this is the most relevant boundary condition for the BAM6QT at Purdue University.

\subsection{Parameter Study}

Parameter studies were performed for both fundamental and subharmonic resonance to identify the most relevant cases and to confirm if indeed fundamental resonance is stronger than the subharmonic resonance. Fundamental resonance or $(\mathrm{K}-$ or Klebanoff-type) breakdown is initiated by a secondary instability mechanism involving a two-dimensional (here an axisymmetric) primary wave $(1,0)$ and a symmetric pair of oblique secondary waves $((1,1)$ and $(1,-1))$ of the same frequency. On the other hand, subharmonic resonance is characterized by a secondary instability mechanism between a primary axisymmetric wave $(2,0)$ and a pair of secondary oblique or three dimensional disturbance waves $(1, \pm 1)$ at half the frequency of the primary wave. Note that in this dissertation, a wave is denoted by $(n, k)$, where $n$ represents the frequency and $k$ the azimuthal wave number, both normalized by the corresponding values of the primary pair of oblique waves. The strength of the fundamental and subharmonic resonance is strongly influenced by the amplitude of the primary wave and the wave angle of the secondary oblique wave pair. For a cone this issue is even more complicated than for a flat-plate, because the wave angle of a disturbance wave changes in the downstream direction. Therefore, a parameter study was performed first to find the most strongly resonating oblique wave pair for both fundamental and subharmonic resonance.

\subsubsection{Fundamental Resonance}

Fundamental resonance can be initiated by forcing the primary wave $(1,0)$ at a large amplitude and the secondary disturbance waves $(1, \pm 1)$ at a low amplitude. The 


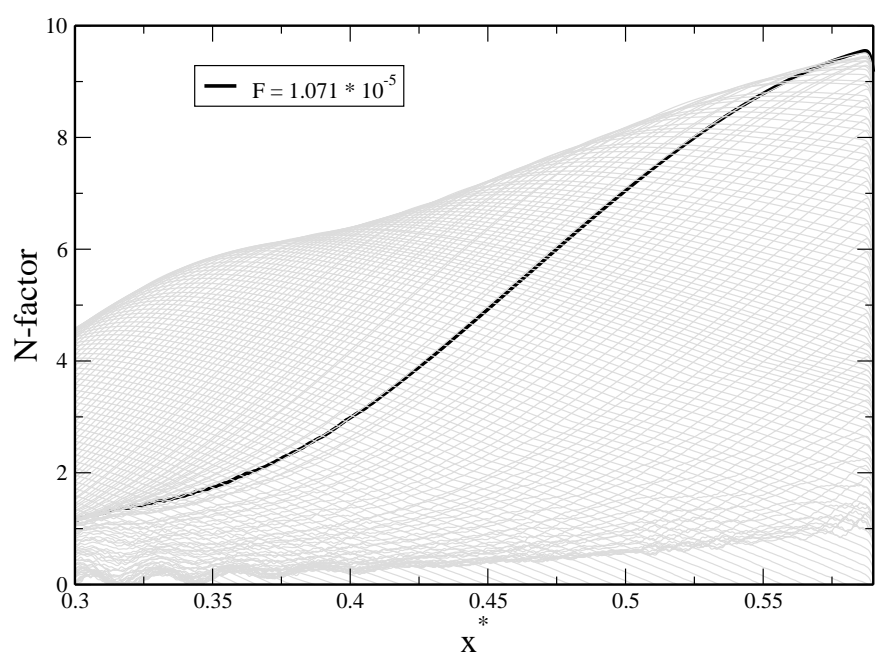

Figure 5.1: N-factor curves for axisymmetric waves $\left(k_{c}=0\right)$ obtained from the lowamplitude wave packet simulation.

dominant (linearly most amplified) axisymmetric wave was chosen as the primary wave. The most amplified primary wave was found from comparing the $\mathrm{N}$-factors for axisymmetric waves for various frequencies. The streamwise distribution of the Nfactors is plotted in figure 5.1 for axisymmetric disturbance waves $\left(k_{c}=0\right)$ as obtained from the low-amplitude wave packet simulation. The N-factor of an instability wave is defined as

$$
N_{n, k_{c}}(x)=\ln \left(\frac{A_{n, k_{c}}(x)}{A_{n, k_{c}, 0}}\right),
$$

where the subscript $n$ and $k_{c}$ denote the frequency and azimuthal wave number of the wave, respectively, $A_{n, k_{c}}(x)$ is the amplitude of the disturbance wave at a certain downstream position and $A_{n, k_{c}, 0}$ is the amplitude of the disturbance wave at its lower neutral point. In figure 5.1 the $\mathrm{N}$-factor reached by the most amplified axisymmetric wave $\left(k_{c}=0\right)$ is about 9.5 . The axisymmetric wave that reached the highest $\mathrm{N}$ factor within the computational domain has a non-dimensional frequency of $F=$ $1.071 \times 10^{-5}\left(f^{*} \approx 210 k H z\right)$ and was therefore chosen as the primary wave $(1,0)$ for a parameter study to determine the most strongly resonating oblique secondary wave pair $(1, \pm 1)$. 

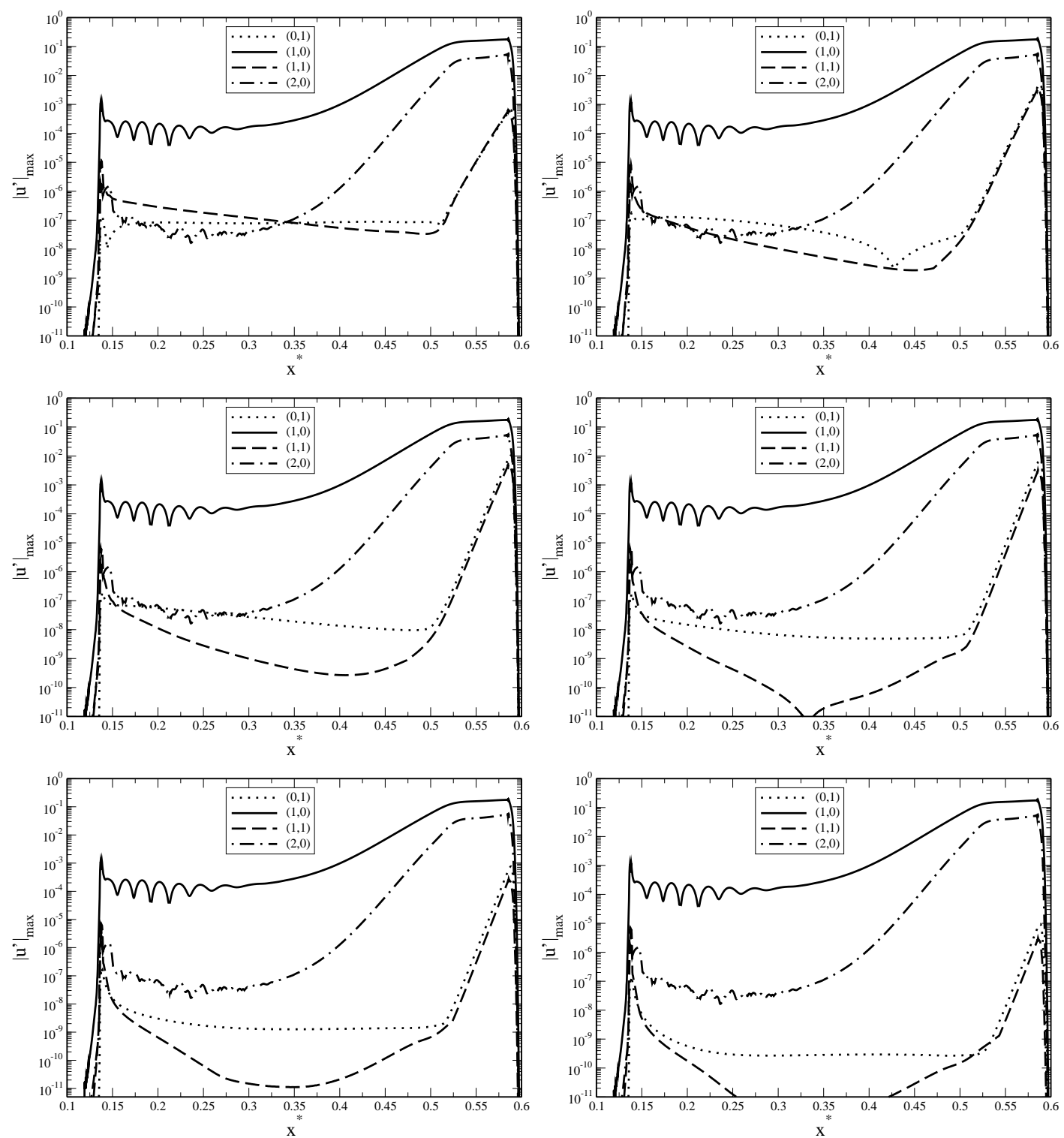

Figure 5.2: Maximum of streamwise velocity disturbance versus downstream distance for the cases with azimuthal wave number $k_{c}=70$ (top left), $k_{c}=90$ (top right), $k_{c}=110$ (center left), $k_{c}=130$ (center right), $k_{c}=150$ (bottom left) and $k_{c}=170$ (bottom right). Shown are selected modes, which play an important role in the early nonlinear stage of fundamental resonance. 


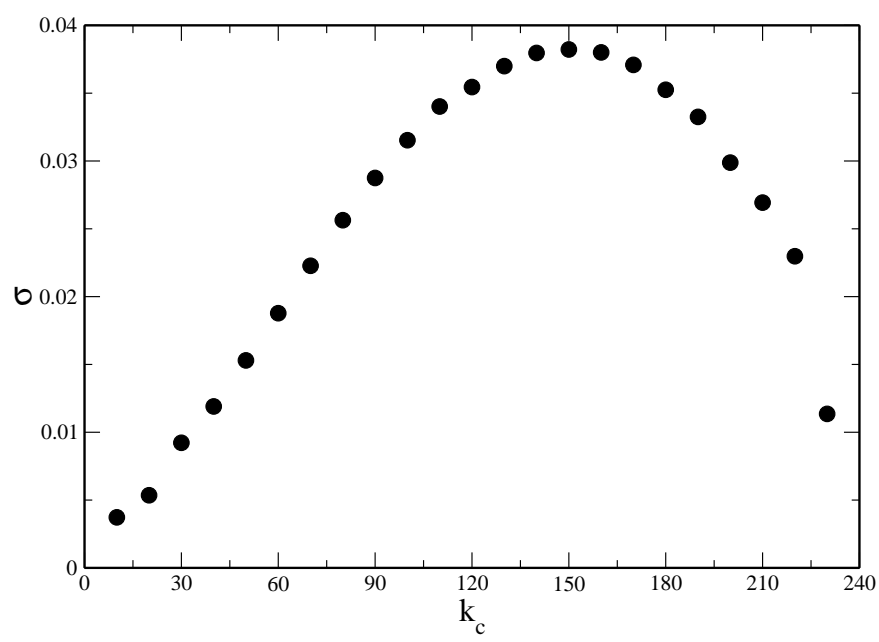

Figure 5.3: Fundamental secondary growth rate $(\sigma)$ as a function of azimuthal mode number $\left(k_{c}\right)$.

A series of low-resolution simulations was performed for several azimuthal wave numbers $\left(k_{c}\right)$ ranging from 10 to 230 . In these simulations the axisymmetric primary wave $(1,0)$ with nondimensional frequency of $F=1.071 \times 10^{-5}\left(f^{*} \approx 210 k \mathrm{~Hz}\right)$ was forced at a moderate amplitude $\left(A_{1,0}=0.1 \%\right.$ of the free-stream velocity $)$ and the secondary oblique waves $(1, \pm 1)$ were forced at a low amplitude $\left(A_{1, \pm 1}=0.001 \%\right.$ of the free-stream velocity). The results obtained from the parameter study are presented in figures 5.2 and 5.3. In figure 5.2, the downstream amplitude development of selected modes is shown for the low-resolution simulation performed for azimuthal mode numbers $k_{c}=70, k_{c}=90, k_{c}=110, k_{c}=130, k_{c}=150$ and $k_{c}=170$. These curves were obtained from plotting the wall-normal maximum of streamwise velocity disturbances. As can be observed in figure 5.2, when the axisymmetric primary wave $(1,0)$ exceeds a certain threshold amplitude, the secondary oblique wave pair $(1, \pm 1)$ and the steady longitudinal vortex mode $(0,1)$ experience strong secondary growth. Figure 5.3 shows the growth rate $(\sigma)$ of the secondary oblique wave pair $(1, \pm 1)$ as a function of azimuthal mode number $\left(k_{c}\right)$. The growth rates $(\sigma)$ were extracted at the streamwise position $x^{*}=0.58 \mathrm{~m}$. A broad band of azimuthal modes experiences 
resonant secondary growth, but according to figure $5.3 \mathrm{a}$, waves with $k_{c}=150$ have the strongest secondary growth rate.

\subsubsection{Subharmonic Resonance}

Subharmonic resonance can be initiated by forcing the primary axisymmetric wave $(2,0)$ at a large amplitude and the secondary disturbance waves $(1, \pm 1)$ at a low amplitude. Note that, a wave is denoted by $(n, k)$ and in particular, $n$ represents the frequency normalized by the value of the primary pair of oblique waves which are at half the frequency of the primary axisymmetric waves here, therefore, the primary axisymmetric wave is labelled as mode $(2,0)$, in contrast to being referred to as mode $(1,0)$ in the case of fundamental resonance. The axisymmetric wave with frequency of $F=1.071 \times 10^{-5}\left(f^{*} \approx 210 \mathrm{kHz}\right)$ that reached the highest $\mathrm{N}$-factor was also chosen as the primary wave for the subharmonic parameter study. Several low-resolution simulations were performed for different azimuthal wave numbers $\left(k_{c}\right)$. As in the case of fundamental resonance parameter studies, the primary axisymmetric wave $(2,0)$ was forced at a moderate amplitude of $A_{1,0}=0.1 \%$ of the free-stream velocity and the secondary oblique waves $(1, \pm 1)$ were forced at a low amplitude of $A_{1, \pm 1}=0.001 \%$ of the free-stream velocity.

The results from the low resolution simulations are presented in figure 5.4 and 5.5. Figure 5.4 shows the downstream development of the wall-normal maximum of streamwise velocity disturbances for selected modes that play an important role in the early nonlinear stage of subharmonic resonance for select cases $\left(k_{c}=60\right.$ and $\left.k_{c}=80\right)$. It can be observed that once the primary wave $(2,0)$ reaches a certain threshold amplitude, the secondary oblique waves $(1, \pm 1)$ experiences secondary growth, which is stronger than its linear growth. However, the results in figure 5.4 indicate that the secondary growth in the case of subharmonic resonance is not strong when compared to the fundamental resonance discussed in the previous section. To confirm, growth 

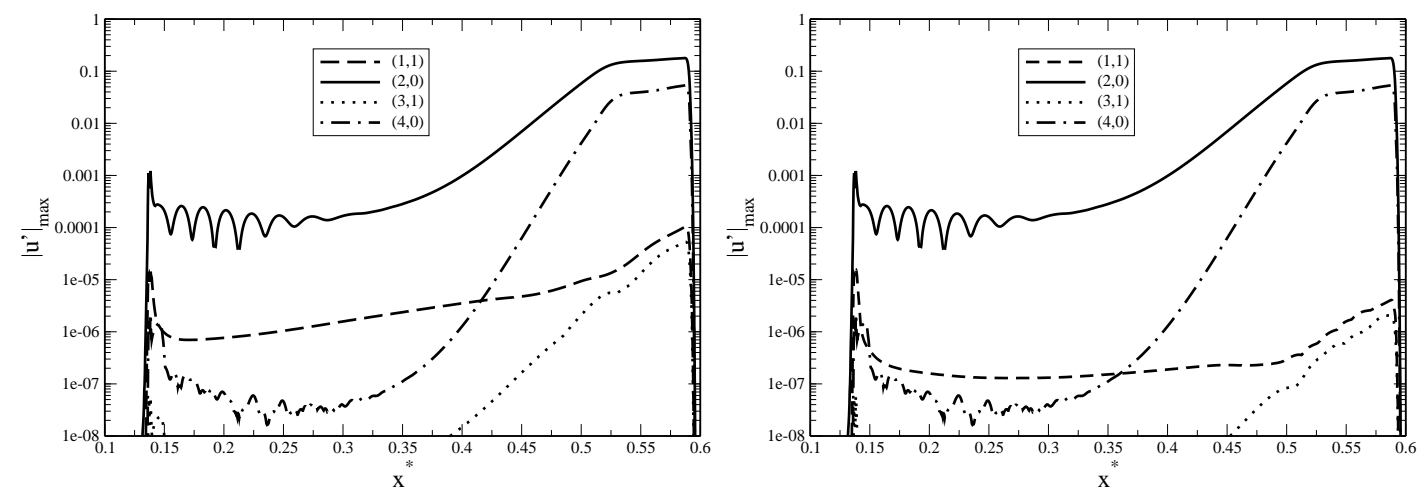

Figure 5.4: Maximum of streamwise velocity disturbance versus downstream distance for the cases with azimuthal wave number $k_{c}=60$ (left) and $k_{c}=80$ (right). Shown are selected modes, which play an important role in the early nonlinear stage of subharmonic resonance.

rates $(\sigma)$ were extracted at the streamwise position $x^{*}=0.55 m$ and are plotted in figure 5.5 as a function of azimuthal mode number $\left(k_{c}\right)$. This plot suggests that secondary oblique waves in the vicinity of azimuthal mode number $k_{c}=60$ experiences the strongest secondary growth. However, when compared to the secondary growth for the case of fundamental resonance (see figure 5.6), the subharmonic secondary growth is considerably weaker.

It will be of great interest to know if the results obtained from these parameter studies can be related to the wave packet simulations discussed in the previous chapter. Therefore, in figure 5.7 the disturbance spectrum obtained from the weakly nonlinear wave packet discussed in the previous chapter is plotted again. The disturbance spectrum is obtained by performing Fourier transformation of the wall-pressure disturbance at a streamwise position $x^{*}=0.58 \mathrm{~m}$. Marked on this spectrum by red and blue circles, are the results from the fundamental and subharmonic parameter studies. It is remarkable how distinct peaks could be identified in the vicinity of azimuthal mode number $k_{c} \sim 150$ and frequency $f^{*} \sim 210 k H z$, identified by the parameter study for the strongest fundamental resonance and also in the vicinity of azimuthal mode number $k_{c} \sim 60$ and frequency $f^{*} \sim 105 k H z$, identified for the maximum subharmonic secondary growth in the parameter study. These results, once 


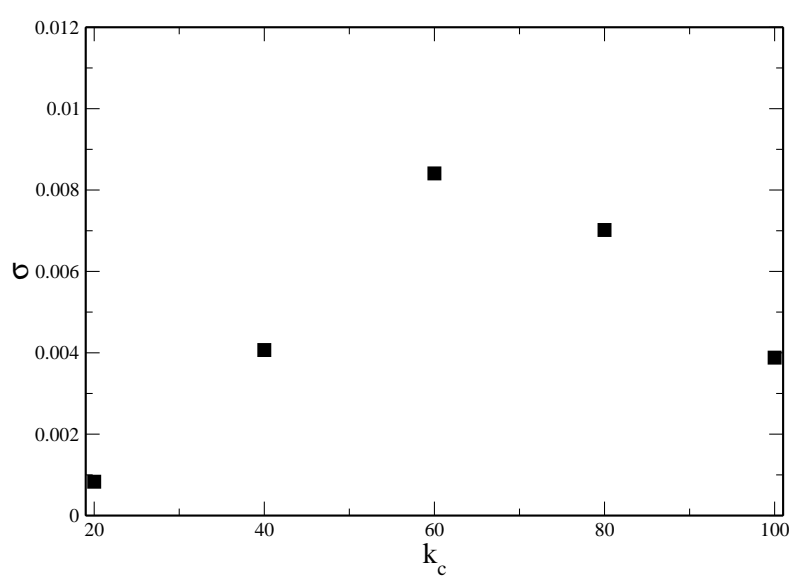

Figure 5.5: Subharmonic secondary growth rate $(\sigma)$ as a function of azimuthal mode number $\left(k_{c}\right)$.

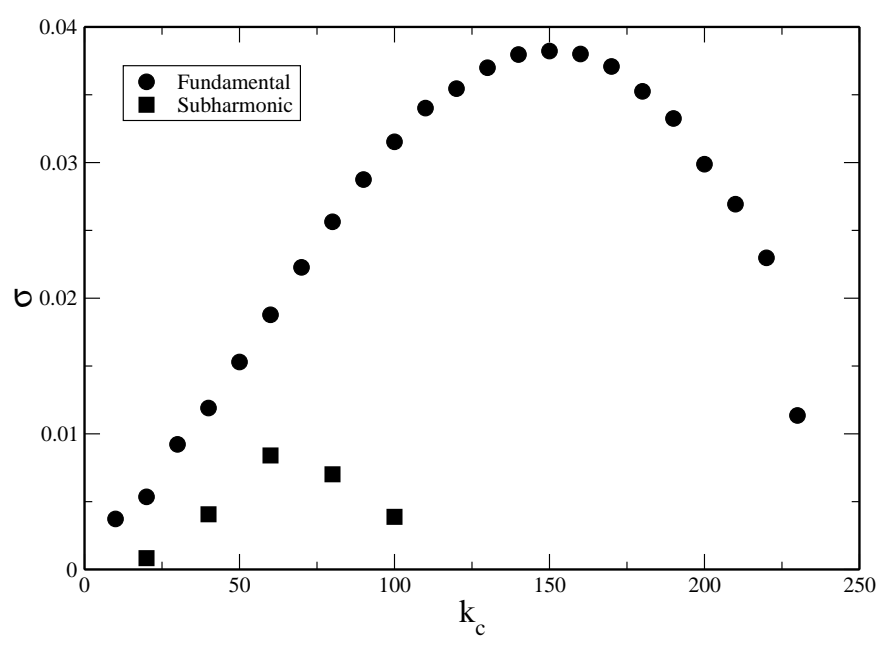

Figure 5.6: Fundamental and subharmonic secondary growth rates $(\sigma)$ as a function of azimuthal mode number $\left(k_{c}\right)$. 


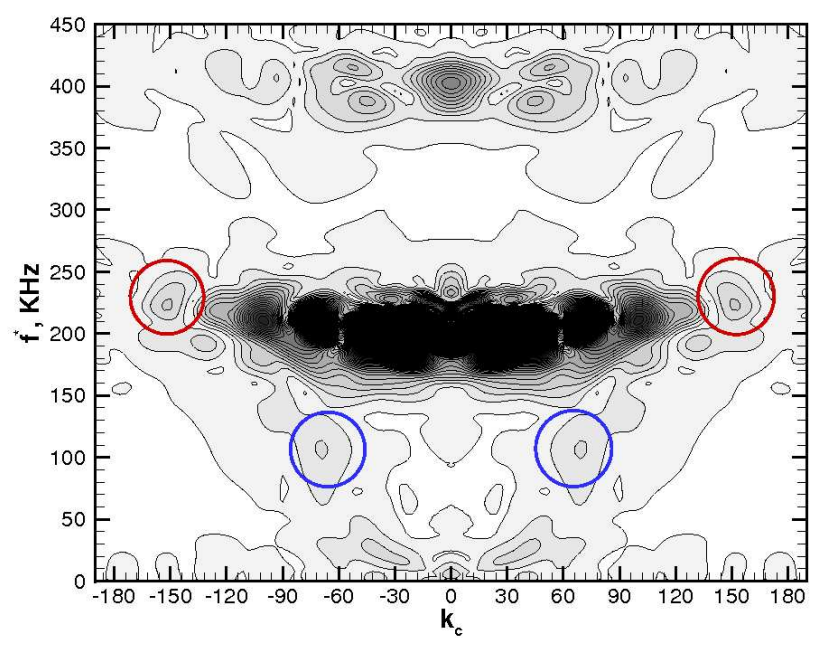

Figure 5.7: Disturbance spectrum in the azimuthal mode number - frequency $\left(k_{c}-f^{*}\right)$ plane from the weakly nonlinear wave packet simulation. Obtained from wall-pressure disturbance at $x^{*}=0.58 \mathrm{~m}$. In the spectrum, red color circle marks a peak in the spectrum that corresponds to the strongest fundamental resonance in the vicinity of $k_{c} \sim 150$ and blue circle marks the peak in the spectrum that corresponds to the strongest subharmonic resonance in the vicinity of $k_{c} \sim 60$.

again confirm the findings of the wave packet investigations, namely that, fundamental resonance is much stronger than the subharmonic resonance for the cone boundary layer at Mach 6.

\subsection{Fundamental Breakdown}

From the parameter study discussed previously for fundamental resonance, oblique waves with azimuthal mode number $k_{c}=150$ were identified as the most strongly resonating oblique waves. Therefore, a set of highly resolved fundamental breakdown simulations were performed with azimuthal mode $k_{c}=150$ for the secondary wave pair. In these simulations, the axisymmetric primary wave $(1,0)$ with a nondimensional frequency of $F=1.071 \times 10^{-5}\left(f^{*} \approx 210 k \mathrm{~Hz}\right)$ was forced with a large amplitude $\left(A_{1,0}=4.0 \%\right.$ of the free-stream velocity). A summary of the forcing parameters used in these fundamental breakdown simulations is provided in table 5.1. 


\begin{tabular}{l|lll} 
& CFUND 1 & CFUND 2 & CFUND 3 \\
\hline \hline$A_{[1,0]}$ & 0.04 & 0.04 & 0.04 \\
$A_{[1, \pm 1]}$ & $1.0 \mathrm{E}-3$ & 0.01 & 0.02 \\
\hline \hline
\end{tabular}

Table 5.1: Forcing parameters for the fundamental breakdown simulations. Note that the notation $[n, k]$ is used in order to identify a particular wave according to its frequency $n$ and its azimuthal wavenumber $k$. $n$ denotes multiples of the fundamental forcing frequency $f^{*}=210 k H z$ and $k$ multiples of the azimuthal wavenumber.

The downstream development of the wall-normal amplitude maximum of the $\mathrm{u}$ velocity disturbance as obtained from simulation CFUND 1 is presented in figure 5.8. Selected modes that play an important role in the early nonlinear stage of a fundamental resonance mechanism are shown. As before, when the axisymmetric primary wave $(1,0)$ reaches a threshold amplitude, the secondary mode $(1,1)$ and the steady longitudinal vortex mode $(0,1)$ starts to grow $\left(\right.$ at $x^{*} \sim 0.47 m$ ) faster than the primary wave and eventually reach the same amplitudes as the primary mode $(1,0)$ at $x^{*} \sim 0.52 m$. Higher harmonics modes $(2,0)$ and $(2,1)$ are also generated. When modes $(1,1)$ and $(0,1)$ approached the amplitude of the primary mode $(1,0)$ all higher modes experienced rapid streamwise growth as the transition process became strongly nonlinear, which is an indication of the onset of the final breakdown to turbulence. Note that in the nonlinear region, the steady longitudinal vortex mode $(0,1)$ has the highest amplitude $\left(0.52 m<x^{*}<0.57 m\right)$. However, close to the end of the computational domain, the steady mode $(0,2)$ has the highest amplitude.

The downstream development of the time- and azimuthally-averaged skin friction coefficient, $C_{f}$, is shown in figure 5.9. The skin friction coefficient is calculated according to

$$
c_{f}=\frac{\left.2 \bar{\mu} \frac{\partial \bar{u}}{\partial y}\right|_{y=0}}{R e},
$$

where the Reynolds number, $R e$, is based on an arbitrary reference length, $L^{*}$ and the flow quantities in the free-stream. Note that symbols with an overbar, $\bar{\phi}$, represent the Reynolds-averaged, i.e. , time- and spanwise-averaged, flow quantities. The skin 
friction curves obtained from the simulations is also compared with the theoretical turbulent value estimated by White (1991),

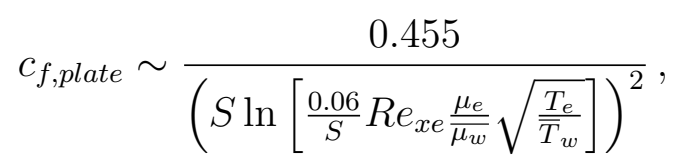

where the subscript $e$ refers to the boundary layer edge conditions and subscript $w$ to wall conditions. The factor $S$ can be obtained from

$$
S=\frac{\left(\frac{\bar{T}_{a w}}{T_{e}}-1\right)^{\frac{1}{2}}}{\arcsin (A)+\arcsin (B)},
$$

with $A$ and $B$ defined as

$$
A=\frac{2 a^{2}-b}{\sqrt{b^{2}+4 a^{2}}} \quad \text { and } \quad B=\frac{b}{\sqrt{b^{2}+4 a^{2}}},
$$

where $a$ and $b$ are given by

$$
a=\sqrt{\frac{\gamma-1}{2} M_{e}^{2} \frac{T_{e}}{\bar{T}_{w}}} \quad \text { and } \quad b=\left(\frac{\bar{T}_{a w}}{\bar{T}_{w}}-1\right),
$$

respectively. Note that $\bar{T}_{a w}$ denotes the adiabatic wall temperature for a turbulent boundary layer at the same flow conditions. Since this value is not known, it has to be estimated using the turbulent recovery factor (White, 1991; Roy \& Blottner, 2006),

$$
r_{\text {turb }} \sim(P r)^{\frac{1}{3}} \sim 0.9
$$

The above relationship used for estimating the skin friction is only valid for a turbulent flat-plate boundary layer. Therefore, a correction for the cone geometry was employed as suggested by van Driest (1952) and White (1991) based on the von Kármán momentum integral: $C_{f, \text { cone }}=G . \times C_{f, p l a t e}$, where $1.1<G<1.15$. Note that the theoretical turbulent skin friction curve in figure 5.9 is calculated with $G=1.15$. The skin friction plotted in figure 5.9 initially follows the laminar curve up to $x^{*} \sim 0.41 \mathrm{~m}$. Then the skin friction increases from the laminar curve towards the turbulent curve. 
However, farther downstream it unexpectedly drops sharply to almost the laminar value before rising again steeply and overshooting the turbulent skin friction curve close to the end of the computational domain. The skin friction curve suggests that the boundary layer is close to a turbulent boundary layer at the end of the computational domain.

A close examination of the results shown in figures 5.8 and 5.9 reveals that the initial rise in skin friction is due to the large amplitude reached by the primary wave $(1,0)$. The first peak in the skin friction corresponds to the streamwise location where the primary wave $(1,0)$ saturates $\left(x^{*} \sim 0.52 m\right)$. This peak in skin friction also roughly coincides with the location where the secondary disturbances (e.g., modes $(1,1)$ and $(0,1))$ reach their largest amplitude levels. As the primary wave starts to decay following the nonlinear saturation, the skin friction dips to almost the laminar value. Then, a much steeper rise in skin friction occurs when all higher modes experience very strong nonlinear amplification. As a result of this steep rise, the skin friction reaches values higher than the theoretical turbulent curve. Note that this particular development of the skin friction is likely due to the "controlled" transition scenario where only a few selected waves are forced. In a "natural" transition scenario this development may be less pronounced or even completely absent. These results raise an interesting question, whether the dip in the skin friction could be influenced by increasing the amplitude of the oblique secondary wave pair $(1, \pm 1)$.

In order to answer this question, two more "controlled" fundamental breakdown simulations, CFUND 2 and CFUND 3, were performed where the forcing amplitudes for the oblique secondary waves were increased to $1.0 \%$ and $2.0 \%$ of the free-stream velocity, respectively. The forcing amplitude for the primary wave was kept the same as in CFUND 1 (4.0\% of the free-stream velocity). For selected modes, the streamwise velocity disturbance development from CFUND 2 and CFUND 3 are plotted in figures 5.10and 5.11. Overall, the amplitude distribution looks very similar to CFUND 1. However, the time- and azimuthally-averaged skin friction coefficient 


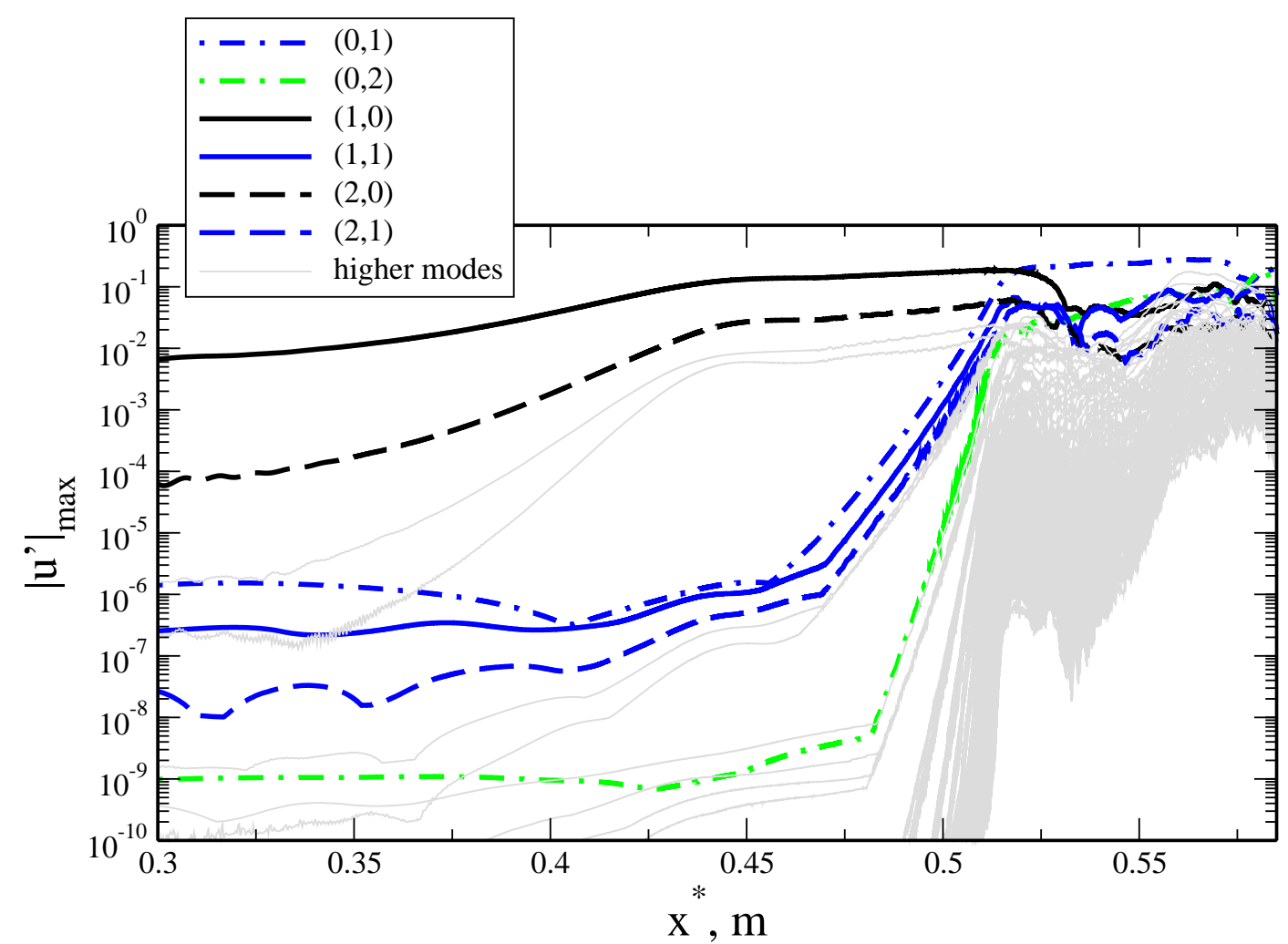

Figure 5.8: Streamwise development of the maximum u-velocity disturbance amplitude from CFUND 1 . Note that when the axisymmetric primary wave $(1,0)$ reaches a threshold amplitude, the secondary mode $(1,1)$ and the steady longitudinal vortex mode $(0,1)$ grow faster than the primary wave and eventually reach the same amplitude as the primary mode. Higher harmonics, modes $(2,0)$ and $(2,1)$ are generated and when modes $(1,1)$ and $(0,1)$ approached the amplitude of the primary mode $(1,0)$ all higher modes experienced rapid streamwise growth. In the nonlinear region the steady longitudinal vortex mode $(0,1)$ has the highest amplitude. Close to the end of the computational domain, however, the steady mode $(0,2)$ has the highest amplitude. 


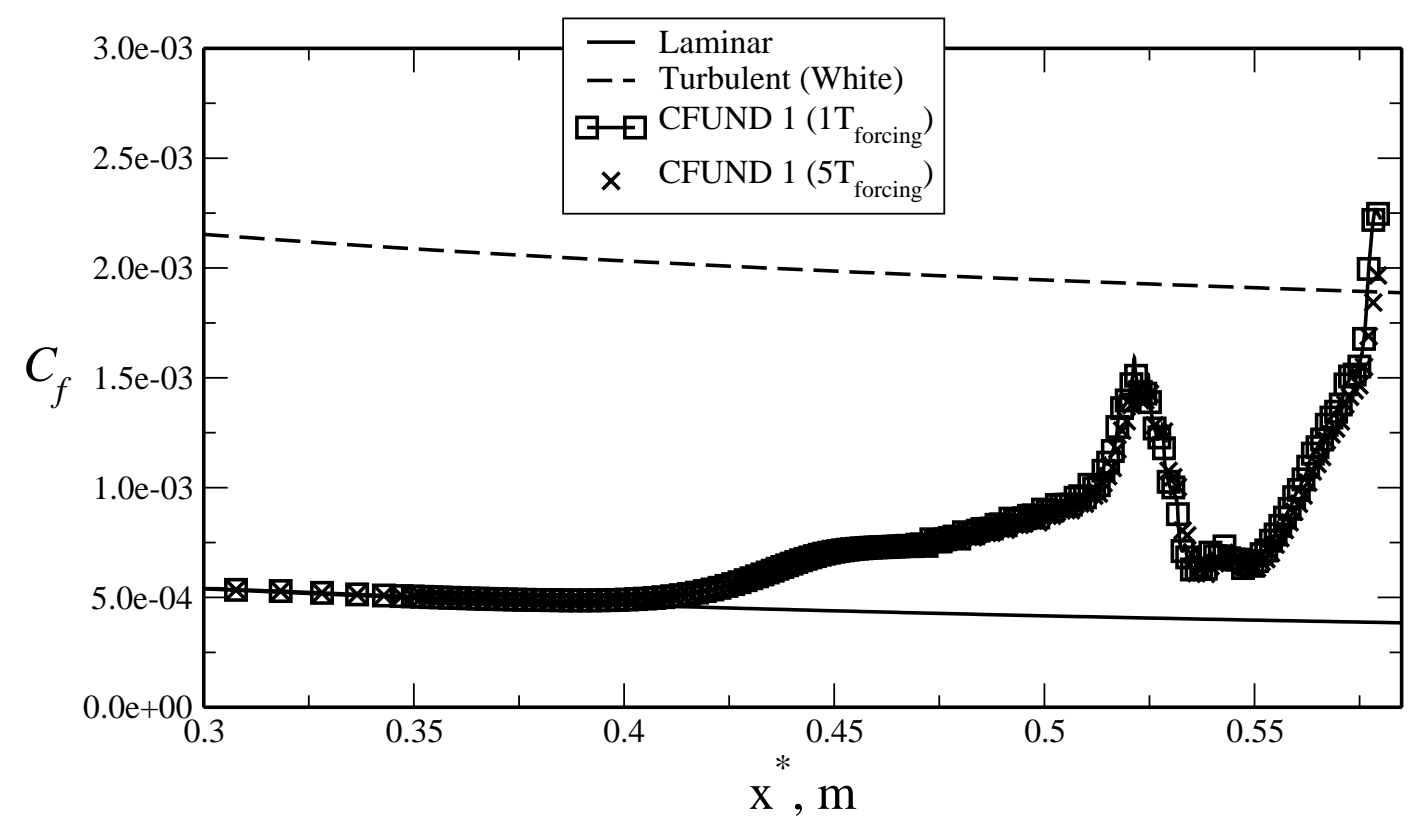

Figure 5.9: Time and azimuthal averaged skin friction coefficient from CFUND 1. Initial rise in skin friction is caused by the large amplitude primary wave $(1,0)$. This is followed by a dip caused by nonlinear saturation of the primary wave $(1,0)$. Then a steeper rise in skin friction occurs when all higher modes experience nonlinear growth. Note that the interval for the time average is indicated by the number of forcing periods. 
from CFUND 2 and CFUND 3 as plotted in figure 5.12 looks slightly different. As the forcing amplitude of the oblique secondary waves are increased, the first peak in the skin friction distribution shifts slightly upstream. This is due to the fact that the secondary growth of the oblique wave starts at a higher amplitude level and consequently reaches nonlinear amplitudes slightly upstream. Therefore, the onset of the rapid streamwise growth of higher modes also shifts slightly upstream with increasing forcing amplitude of secondary waves. In CFUND 2 and CFUND 3, as the primary wave is forced with the same amplitude as in CFUND 1, the location of transition onset remains the same for all three cases. This confirms that the initial rise in skin friction (onset of transition) is due to the large amplitude of the primary wave $(1,0)$.

Isocontours of the time-averaged wall heat flux $(d T / d y)$ and skin friction are shown in figures 5.13 and 5.14. Remarkable are the streamwise alligned "hot" streaks. These streaks look similar to the streamwise streaks observed in the Purdue experiments using temperature sensitive paint (Berridge et al., 2010). The qualitative similarity of the "hot" streaks observed in our simulations and the streamwise streaks observed in the Purdue experiments for a flared cone (see figure $7 \mathrm{~b}$ in Berridge et al. (2010)), may be an indication that fundamental resonance might have played a role in the unforced "natural" transition experiments at Purdue.

Identification of dominant flow structures and their streamwise development will give further insight into the underlying physical mechanisms of the transition process. Instantaneous flow structures identified by the Q-criterion is shown in figures 5.15, 5.16 and 5.17. Q is the second invariant of the velocity gradient tensor and can be computed using:

$$
Q=\frac{1}{2}\left(W_{i j} W_{i j}-S_{i j} S_{i j}\right)
$$

where

$$
S_{i j}=\frac{1}{2}\left(\frac{\partial u_{i}}{\partial x_{j}}+\frac{\partial u_{j}}{\partial x_{i}}\right), \quad W_{i j}=\frac{1}{2}\left(\frac{\partial u_{i}}{\partial x_{j}}-\frac{\partial u_{j}}{\partial x_{i}}\right) .
$$




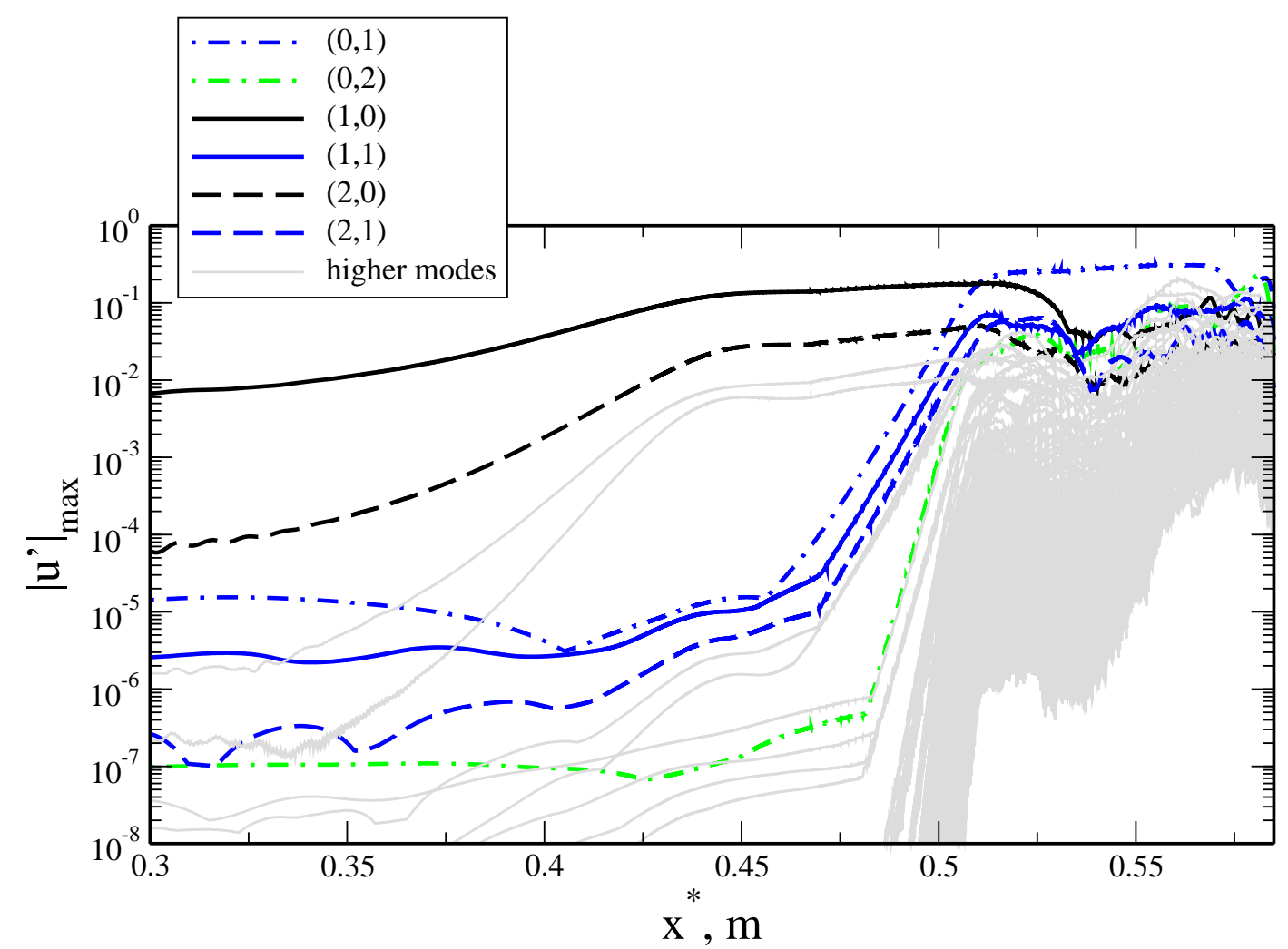

Figure 5.10: Streamwise development of the maximum u-velocity disturbance amplitude from CFUND 2. Note that when the axisymmetric primary wave $(1,0)$ reaches a threshold amplitude, the secondary mode $(1,1)$ and the steady longitudinal vortex mode $(0,1)$ grow faster than the primary wave and eventually reach the same amplitude as the primary mode. Higher harmonics, modes $(2,0)$ and $(2,1)$ are generated and when modes $(1,1)$ and $(0,1)$ approached the amplitude of the primary mode $(1,0)$ all higher modes experienced rapid streamwise growth. In the nonlinear region the steady longitudinal vortex mode $(0,1)$ has the highest amplitude. Close to the end of the computational domain, however, the steady mode $(0,2)$ has the highest amplitude. Overall the observations were very similar to what was seen for CFUND 1. 


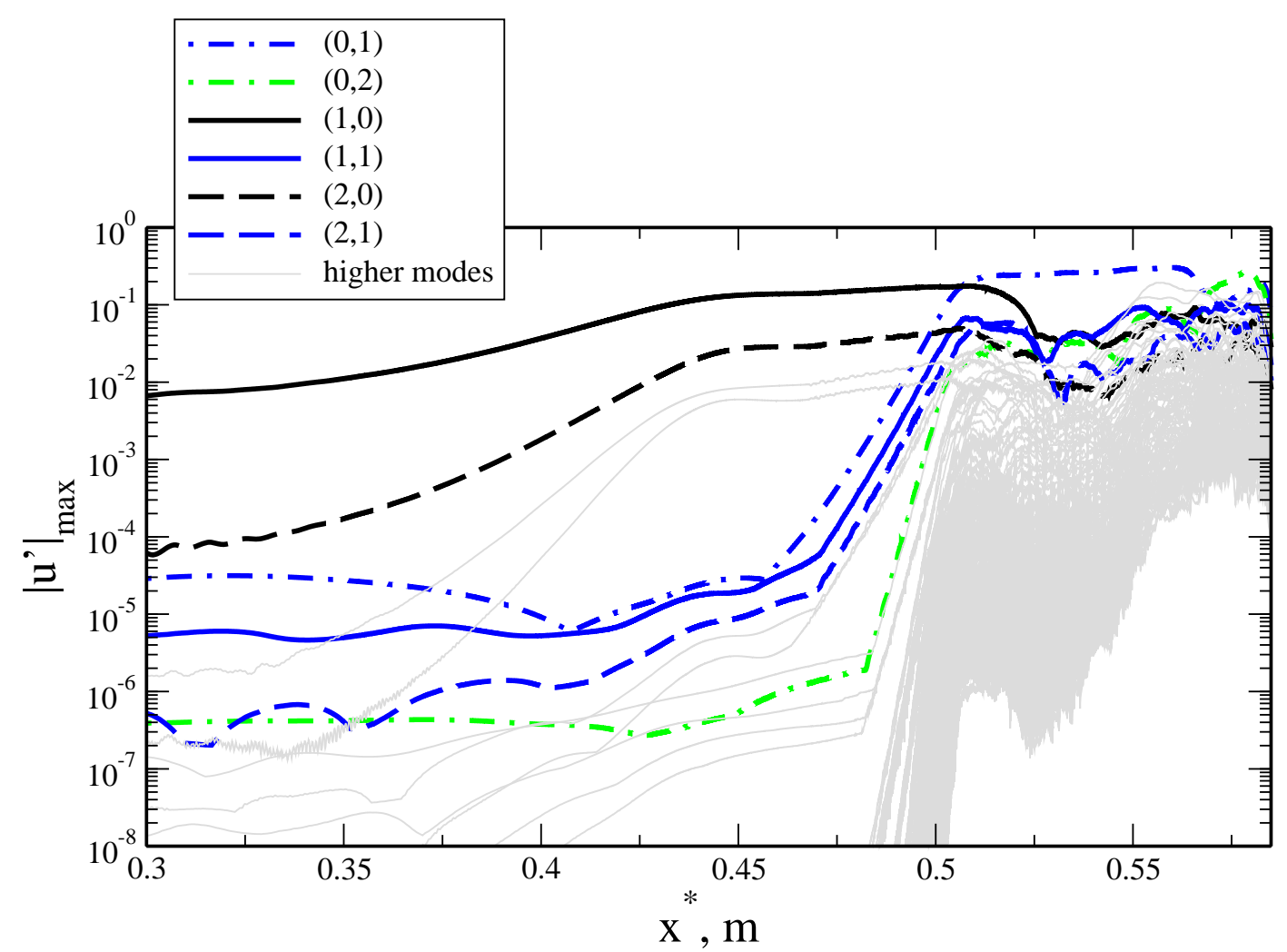

Figure 5.11: Streamwise development of the maximum u-velocity disturbance amplitude from CFUND 3. Note that when the axisymmetric primary wave $(1,0)$ reaches a threshold amplitude, the secondary mode $(1,1)$ and the steady longitudinal vortex mode $(0,1)$ grow faster than the primary wave and eventually reach the same amplitude as the primary mode. Higher harmonics, modes $(2,0)$ and $(2,1)$ are generated and when modes $(1,1)$ and $(0,1)$ approached the amplitude of the primary mode $(1,0)$ all higher modes experienced rapid streamwise growth. In the nonlinear region the steady longitudinal vortex mode $(0,1)$ has the highest amplitude. Close to the end of the computational domain, however, the steady mode $(0,2)$ has the highest amplitude. Overall the observations were very similar to what was seen for CFUND 1 and 2. Note that close to the end of the computational domain the steady mode $(0,2)$ is much more stronger than in for CFUND 1 and 2 . 


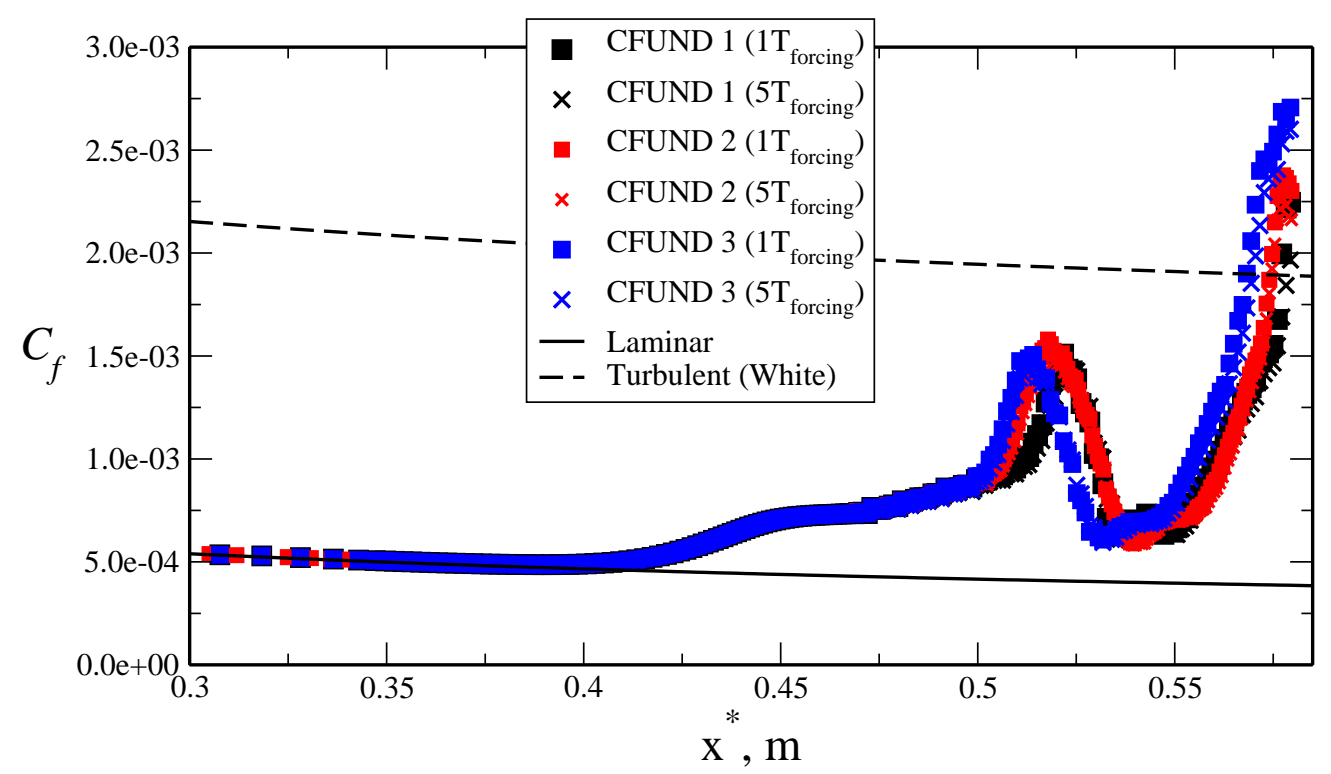

Figure 5.12: Time- and azimuthally-averaged skin friction coefficient from CFUND 1, CFUND 2 and CFUND 3. Initial rise in skin friction is caused by the large amplitude primary wave $(1,0)$. This is followed by a dip caused by nonlinear saturation of the primary wave $(1,0)$. Then a steeper rise in skin friction occurs when all higher modes experience nonlinear growth. The same observation has been made for all three cases, however, as the forcing amplitude of the oblique secondary waves are increased for CFUND 2 and CFUND 3, the first peak in the skin friction distribution shifts slightly upstream. Note that the interval for the time average is indicated by the number of forcing periods. 

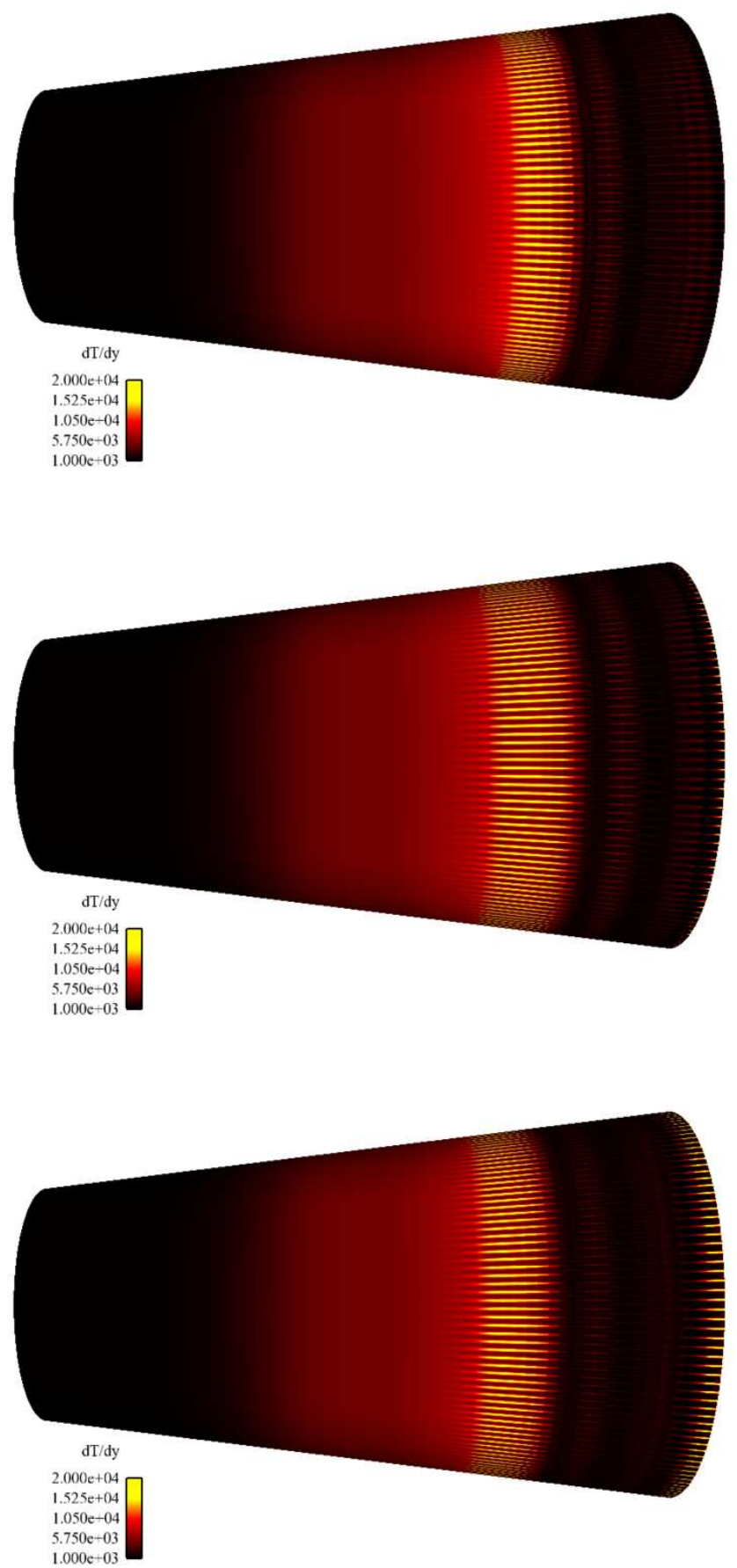

Figure 5.13: Time-averaged heat transfer $(d T / d y)$ at the wall from CFUND 1 (top), CFUND 2 (center) and CFUND 3 (bottom). The streamwise alligned "hot" streaks observed in our simulations looks qualitatively similar to the streamwise streaks observed in the Purdue experiments using temperature sensitive paints for a flared cone (Berridge et al., 2010). 

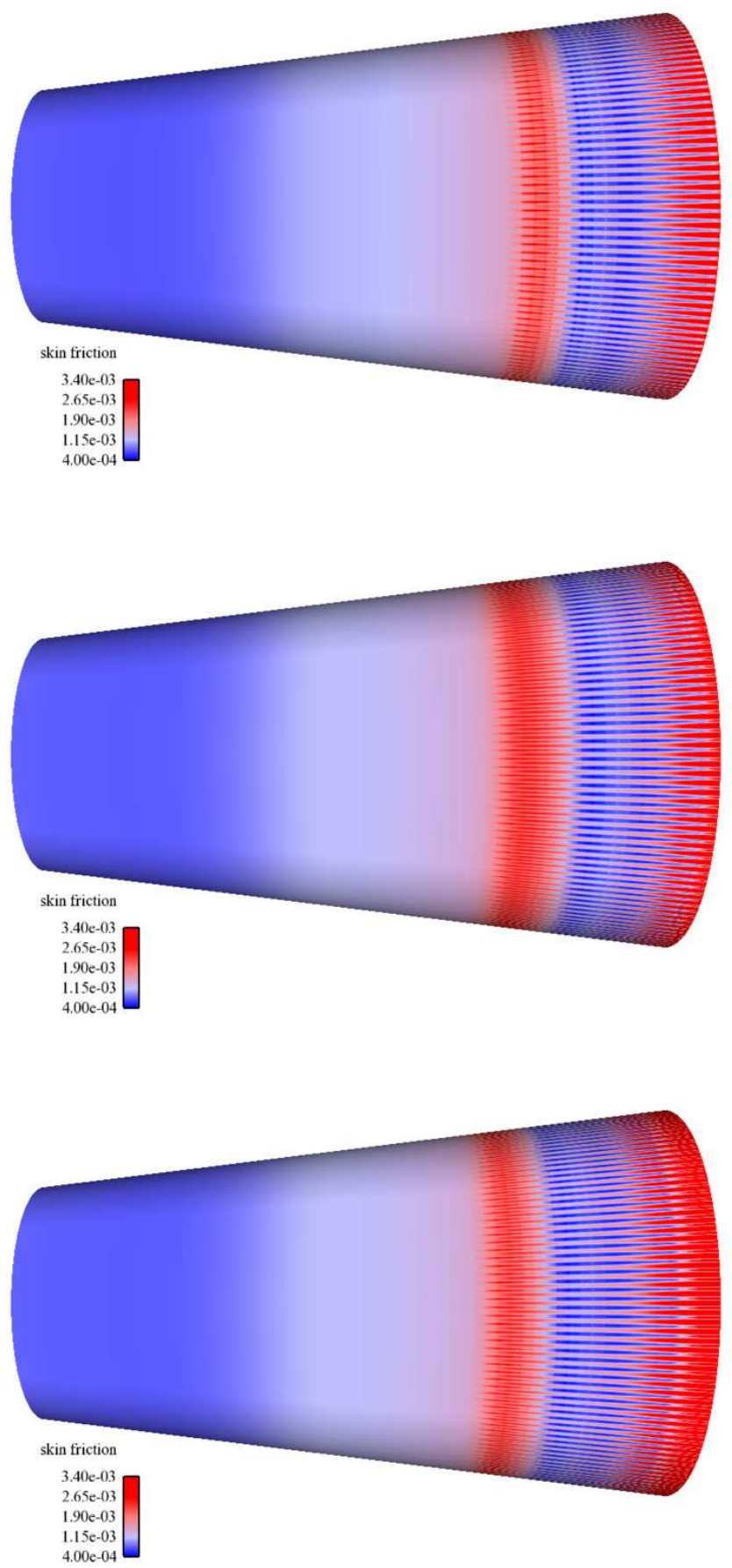

Figure 5.14: Time-averaged skin friction from CFUND 1 (top), CFUND 2 (center) and CFUND 3 (bottom). The streamwise alligned "hot" streaks observed in our simulations looks qualitatively similar to the streamwise streaks observed in the Purdue experiments using temperature sensitive paints for a flared cone (Berridge et al., 2010). 

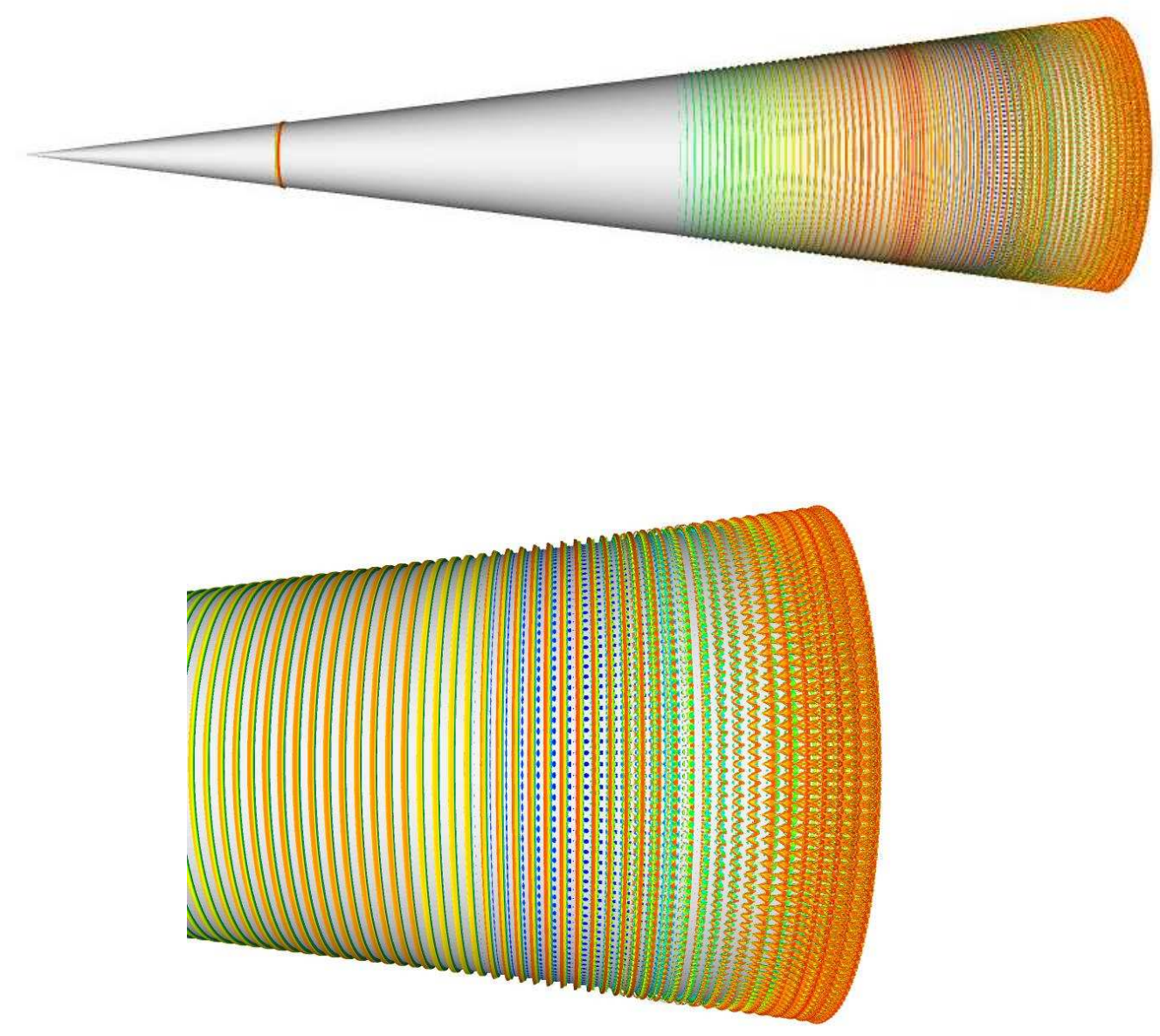

Figure 5.15: Visualization of flow structures by isosurface of $\mathrm{Q}$ criterion from CFUND 3. (a) Full view of the cone (top) and (b) close-up view of the last downstream part of the cone (bottom). The isosurface is colored using the streamwise velocity magnitude. 

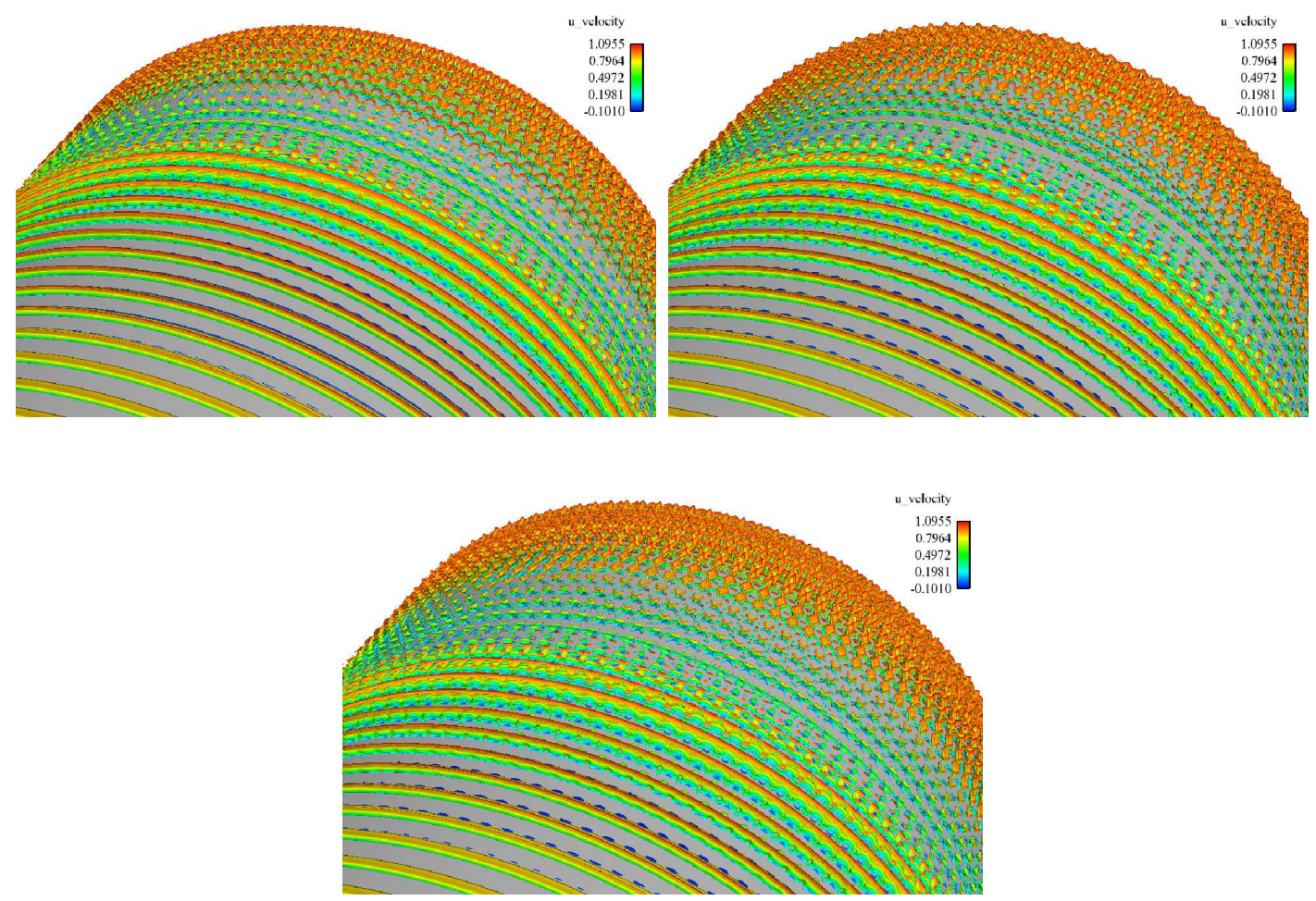

Figure 5.16: Visualization of flow structures by isosurface of Q criterion. Shown are perspective view of isosurface of $\mathrm{Q}$ criterion from CFUND 1 (top left), CFUND 2 (top right) and CFUND 3 (bottom). The isosurface is colored using the streamwise velocity magnitude. 


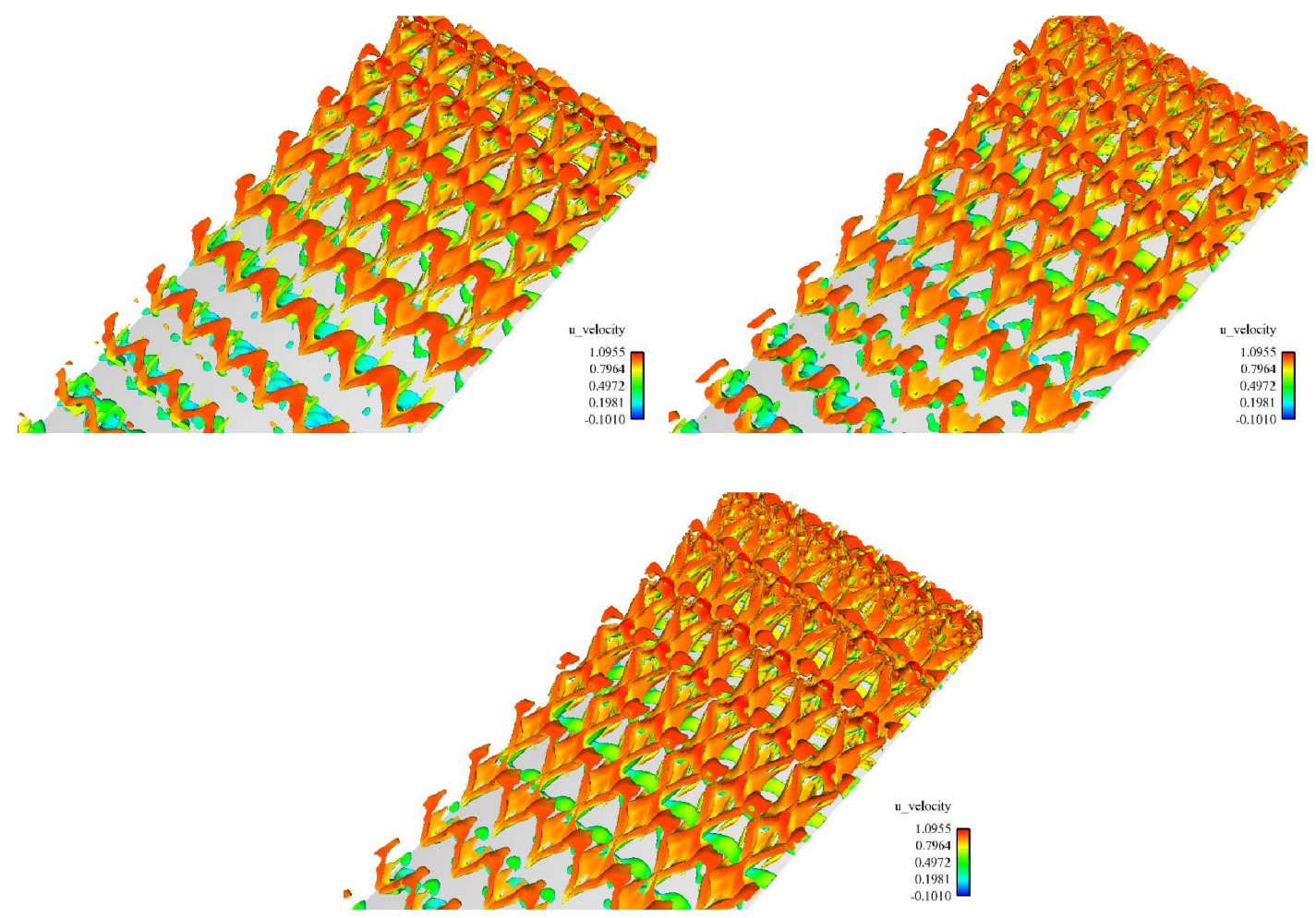

Figure 5.17: Visualization of flow structures by isosurface of $\mathrm{Q}$ criterion. Shown are close-up view of isosurface of $\mathrm{Q}$ criterion from CFUND 1 (top left), CFUND 2 (top right) and CFUND 3 (bottom). The isosurface is colored using the streamwise velocity magnitude. 
Positive values of $\mathrm{Q}$ reveal flow regions where the magnitude of the rotation rate, $W_{i j}$, exceeds the magnitude of the strain rate, $S_{i j}$. In figure 5.15 the flow structures from CFUND 3 are plotted. The flow structures reveal the typical evolution of a K-type breakdown: During the initial stage of the transition process the dominant wave is axisymmetric. Once nonlinear interactions cause the oblique secondary waves to amplify rapidly, the dominant axisymmetric waves become modulated in the azimuthal direction. The interaction of the dominant axisymmetric wave and finite amplitude oblique waves leads to the formation of $\Lambda$-vortices. The $\Lambda$-vortices appear in an aligned pattern because the primary axisymmetric wave and the secondary oblique waves have the same frequency.

Additionally, the evolution of the flow structures during the final stage of transition can be better observed in the perspective and close-up view of figure 5.16 and 5.17. The aligned arrangement of the $\Lambda$-vortices can be clearly identified. The tips of the $\Lambda$-vortices begin lifting away from the surface while the legs remain close to the wall. Hairpin-shaped vortices start to appear on the tip of the $\Lambda$-vortices. Eventually these structures breakdown to smaller scales as the flow starts to become turbulent close to the end of the computational domain.

The time- and azimuthally-averaged streamwise velocity profiles plotted in wall coordinates can provide more insight on how far transition has progressed. The streamwise velocity is rescaled with the friction velocity and for compressible flow, the velocity is typically transformed using the van Driest transformation,

$$
u_{c}^{+}=\int_{0}^{\bar{u}} \sqrt{\frac{\bar{T}_{w}}{\bar{T}}} d u
$$

The transformed velocity profiles corresponding to several streamwise positions are shown in figure 5.18. The profiles are obtained from CFUND 1, CFUND 2 and CFUND 3. Note that the dotted lines represent the theoretical curves for the linear 
sublayer and the logarithmic overlap region, which follows the form

$$
u^{+}=y^{+}, u^{+}=\frac{1}{\kappa} \ln \left(y^{+}\right)+C .
$$

For the von Kármán constant, $\kappa$, typically a value of $\kappa=0.41$ is used while the constant $C$ is about 5.2 (Roy \& Blottner, 2006). For an upstream location, $x^{*}=0.4 m$ the velocity profile looks like a laminar profile, as this location is upstream of the transition onset location $\left(x^{*} \sim 0.41 m\right)$. For locations close to the peak in the skin friction $\left(x^{*}=0.57 m\right)$, the van Driest transformed velocity approaches the theoretical curves. This indicates that the flow is starting to become turbulent. The profiles from the three cases showed similar characteristics.

\subsection{Oblique Breakdown}

In addition to providing evidence for the presence of a strong fundamental resonance mechanism the wave packet simulations have also indicated a possible presence of a strong oblique breakdown mechanism in the nonlinear transition regime of a sharp cone boundary layer at Mach 6. Oblique breakdown is not a secondary instability mechanism in the classical sense. Rather than a primary disturbance causing nonlinear growth of a lower amplitude secondary disturbance, oblique breakdown is initiated only by a pair of oblique disturbance waves with identical, but opposite, wave angles - modes $(1, \pm 1)$. The pair of oblique disturbances grows and eventually reaches amplitudes large enough to cause nonlinear amplification of numerous higher modes. As mentioned previously in Section 1, the oblique breakdown mechanism was first discovered for supersonic flows by Thumm (1991) and Fasel et al. (1993) in DNS of flat-plate boundary layer transition at Mach 1.6. They showed that in the nonlinear region the primary pair of oblique waves transfer energy to a steady mode (longitudinal vortex) with twice the spanwise wave number of the primary oblique waves. The growth of this steady mode results from a wave-vortex triad $((1,1),(1,-1)$ and $(0,2))$ 

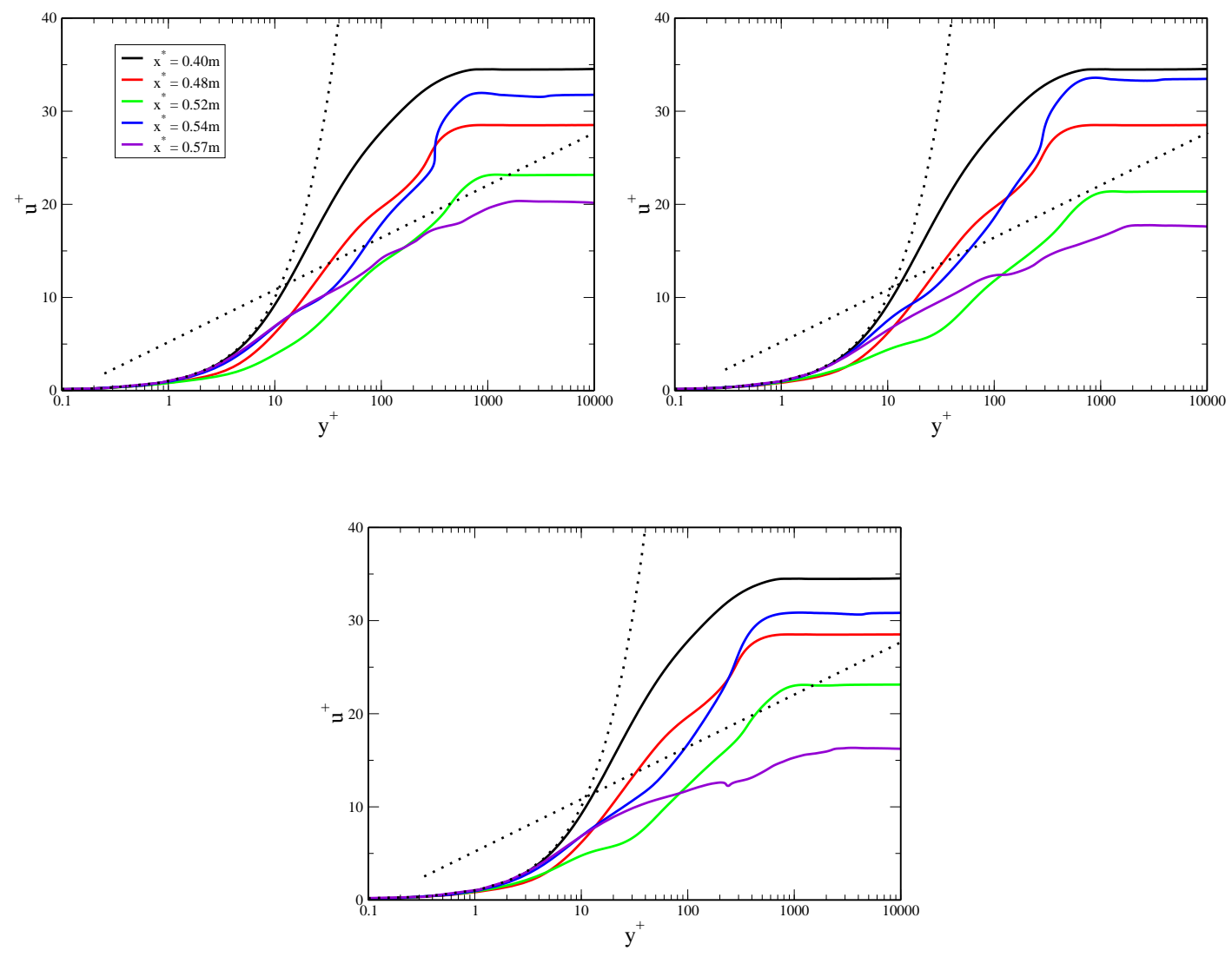

Figure 5.18: Van Driest transformed streamwise velocity profiles normalized with the friction velocity for different streamwise positions from CFUND 1 (top left), CFUND 2 (top right) and CFUND 3 (bottom). For streamwise position close to the peak in the skin friction $\left(x^{*}=0.57 m\right)$, the van Driest transformed velocity approaches the theroretical curves. The dotted lines indicate the linear sublayer and the law of the wall. 

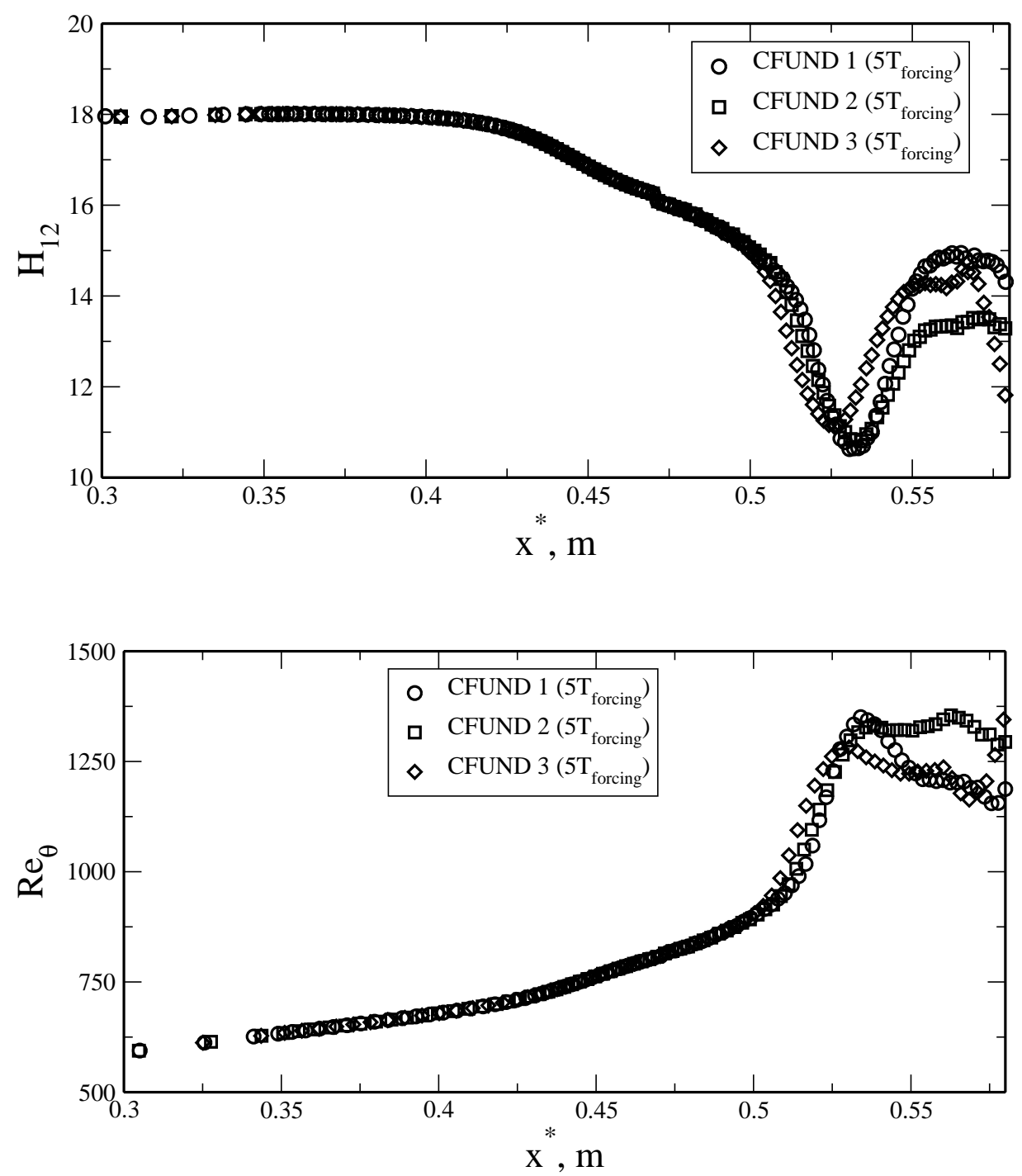

Figure 5.19: Downstream development of select mean-flow properties: (a) shape factor, $H_{12}$ (top) and (b) Reynolds number based on momentum thickness, $R e_{\theta}$ (bottom) from CFUND 1, CFUND 2 and CFUND 3. The time average was obtained for five forcing periods ( $\left.T_{\text {forcing }}\right)$. 

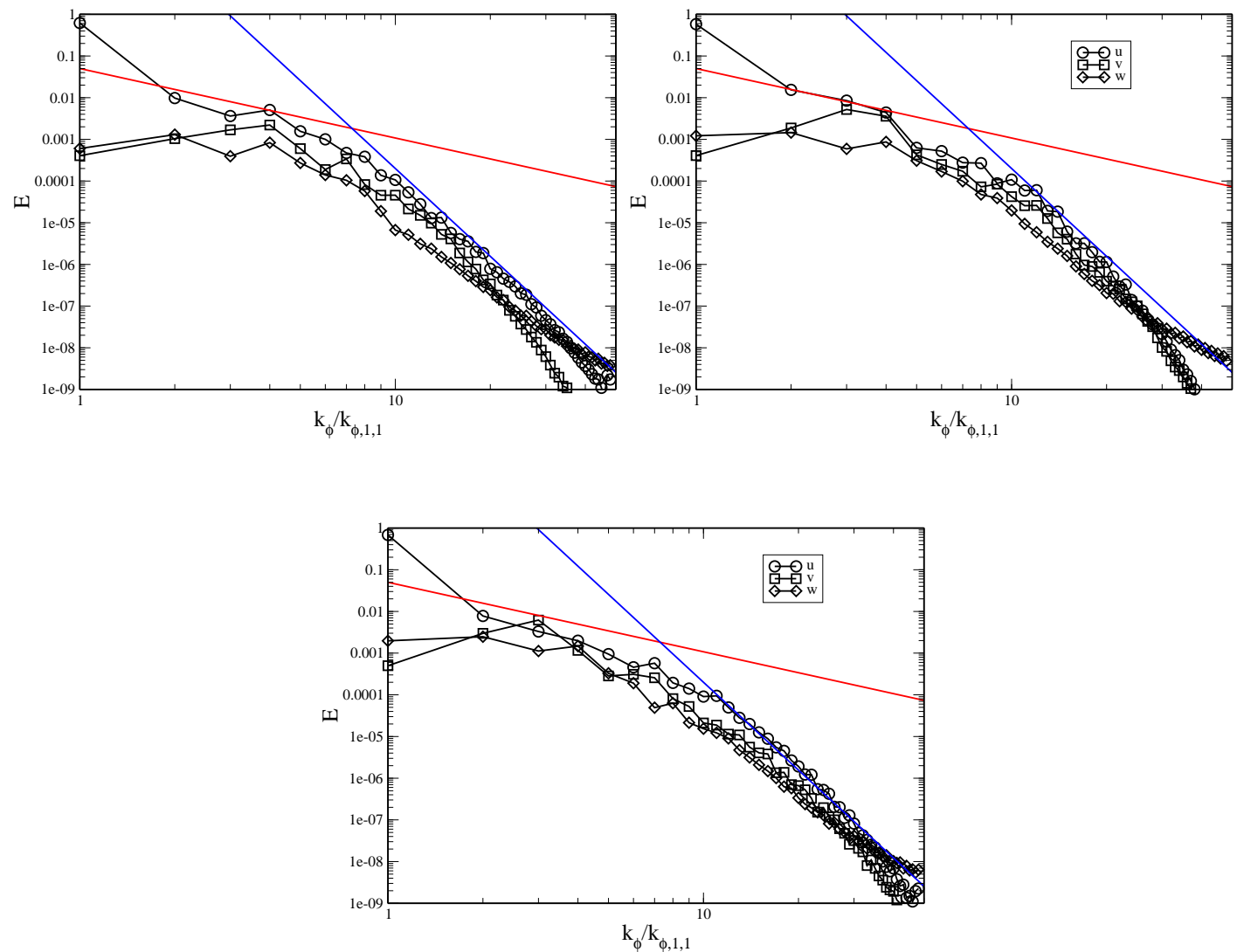

Figure 5.20: Energy spectra versus $k_{\phi}$ for $u, v$ and $w$ from CFUND 1 (top left), CFUND 2 (top right) and CFUND 3 (bottom) at $x^{*}=0.57 \mathrm{~m}$. The red and blue lines indicate the theoretical estimate for the decay of the energy spectrum: $E \sim k_{\phi}^{-5 / 3}$ (red line) and $E \sim k_{\phi}^{-7}$ (blue line). 


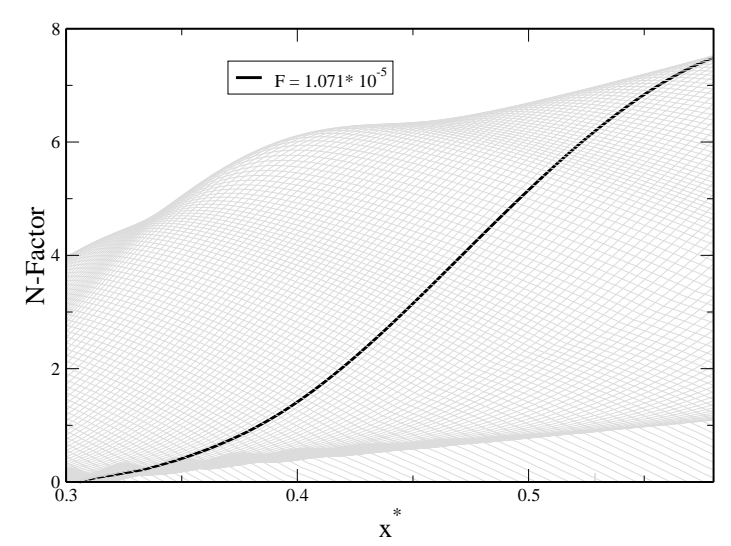

Figure 5.21: $\mathrm{N}$-factor curves for oblique waves with azimuthal wave number $k_{c}=20$. The N-factor curves were obtained from the low-amplitude wave packet simulation.

and is considered to be the dominant mechanism early in the transition process. As transition progresses, higher harmonics are generated and the spectrum is rapidly broadened, especially in the spanwise direction. Although for Mach numbers greater than 4, two-dimensional second-mode disturbances are most amplified. Low-wavenumber second-mode oblique waves experience similar linear growth. Husmeier \& Fasel (2007) demonstrated that oblique breakdown of shallow angle waves may also be relevant for hypersonic Mach numbers.

In order to compare with the previously discussed fundamental breakdown simulations and to find out if oblique breakdown can indeed be a viable path to complete breakdown to turbulence a highly resolved oblique breakdown simulation was performed. The disturbance spectrum obtained from wave packet simulations discussed in Chapter 4 showed strong peaks for shallow oblique waves with azimuthal wave numbers $k_{c} \pm 20$. Consequently, waves with this azimuthal wave numbers were forced in the present simulation in order to initiate the transition process via oblique breakdown. The frequency was chosen to be $F=1.071 \times 10^{-5}\left(f^{*} \approx 210 k H z\right)$, because it is linearly the most amplified frequency for the azimuthal mode number $k_{c}=20$ as shown in figure 5.21. Note that the dominant axisymmetric wave $\left(k_{c}=0\right)$ and the dominant oblique wave with $k_{c}=20$ have about the same frequency. 
The downstream development of the wall-normal amplitude maximum of the $\mathrm{u}$ velocity disturbances as obtained from the oblique breakdown simulation is presented in figure 5.22. Shown are the modes that play an important role in the early nonlinear stages of transition via an oblique breakdown mechanism. Highlighted are the signature modes of oblique breakdown: the initially forced mode $(1,1)$, the nonlinearly generated steady (longitudinal) modes $(0,2),(0,4),(0,6)$ and higher mode $(1,3)$. In contrast to the "classical" breakdown processes (K-type or N/H-type), in oblique breakdown, it is not possible to separate the linear and the nonlinear region with distinct downstream locations. This is mainly because of the different nonlinear mechanisms that are responsible for the growth of three-dimensional disturbances. The longitudinal mode $(0,2)$ and the traveling modes $(2,0)$ and $(2,2)$ are generated by the first level of nonlinear interaction. These are a direct consequence of the nonlinear interaction of the pair of oblique waves with themselves. The second level of nonlinear interactions produces higher harmonics, e.g., mode $(1,3)$. Despite being generated by nonlinear interactions, both the steady modes $(0,2),(0,4)$ and the higher traveling modes $(1,3)$ and $(1,5)$ experience a stronger amplification than the primary wave $(1,1)$, and eventually reach the same amplitude level. Once a certain threshold amplitude is reached, nonlinear mechanisms eventually trigger the transition process, then all higher modes experienced stronger streamwise growth as the transition process becomes highly nonlinear, which leads to the onset of the final breakdown to turbulence. In the nonlinear regime, the steady longitudinal vortex mode $(0,2)$ has the highest amplitude (at $\left.x^{*} \sim 0.47 m\right)$, mode $(0,4)$ has the second highest amplitude and mode $(0,6)$ has the third highest. Close to the end of the computational domain, however, it is mode $(0,4)$ and other higher modes that have the highest amplitude. The transition process seem to be dominated by the generation and final breakdown of these steady (modes) longitudianal vortices.

The downstream development of the time-- and azimuthally averaged skin friction coefficient, $C_{f}$ is shown in figure 5.23. The skin friction initially follows the laminar 


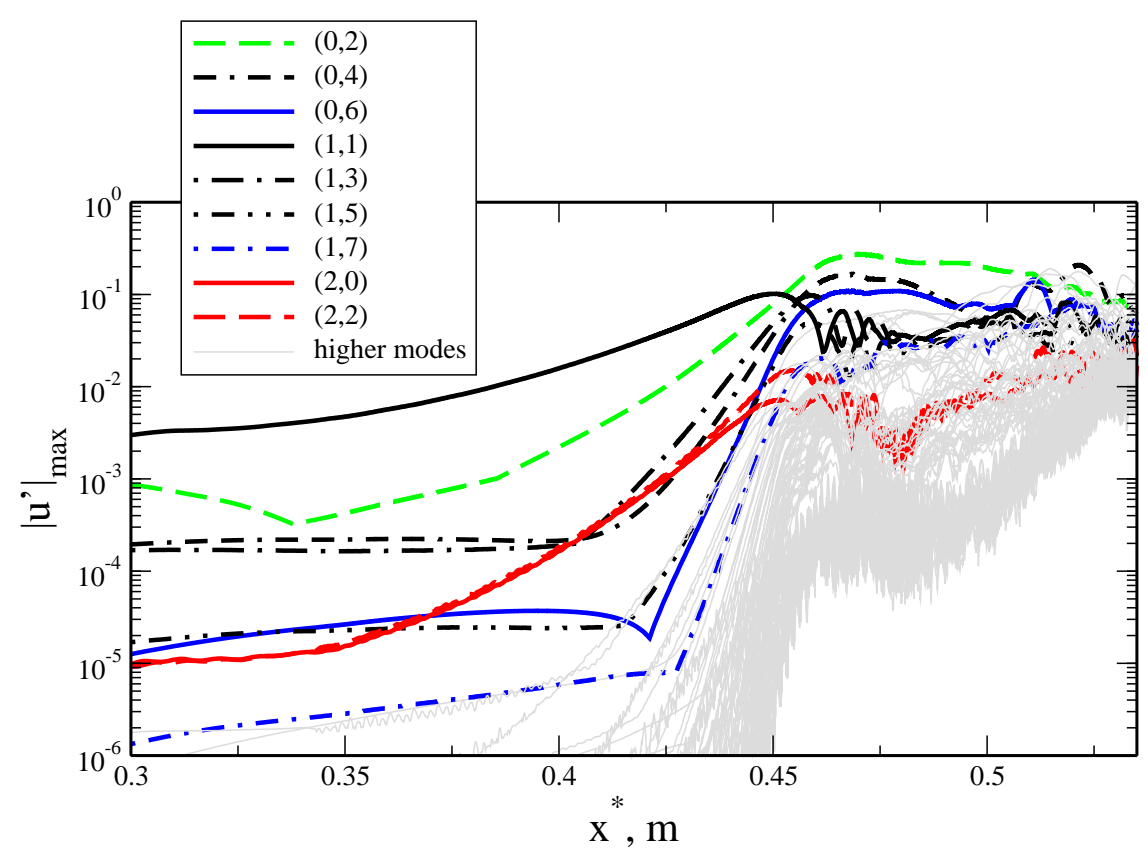

Figure 5.22: Streamwise development of the maximum u-velocity disturbance amplitude from the oblique breakdown simulation.

curve up to $x^{*} \sim 0.44 m$. Then there is a small initial rise and then a dip in the skin friction coefficient, similar to the initial rise and fall observed in the fundamental breakdown simulations. As in the case of fundamental breakdown the initial rise in skin friction is related to the high amplitude primary wave. However, the rise is much less pronounced for oblique breakdown, which could be due to the lower amplitude of the primary wave $(1,1)$ because the oblique primary wave is not as strongly amplified as axisymmetric disturbances. Farther downstream, however, the skin friction rises again steeply and overshoots the turbulent skin friction curve close to the end of the computational domain.

In figure 5.24, isocontours of the time-averaged wall-heat flux $(d T / d y)$ and skin friction obtained from the oblique breakdown simulation is shown. As for the fundamental breakdown simulations, streamwise arranged "hot" streaks were also observed for oblique breakdown in both wall-heat flux and skin friction. However, these streaks 


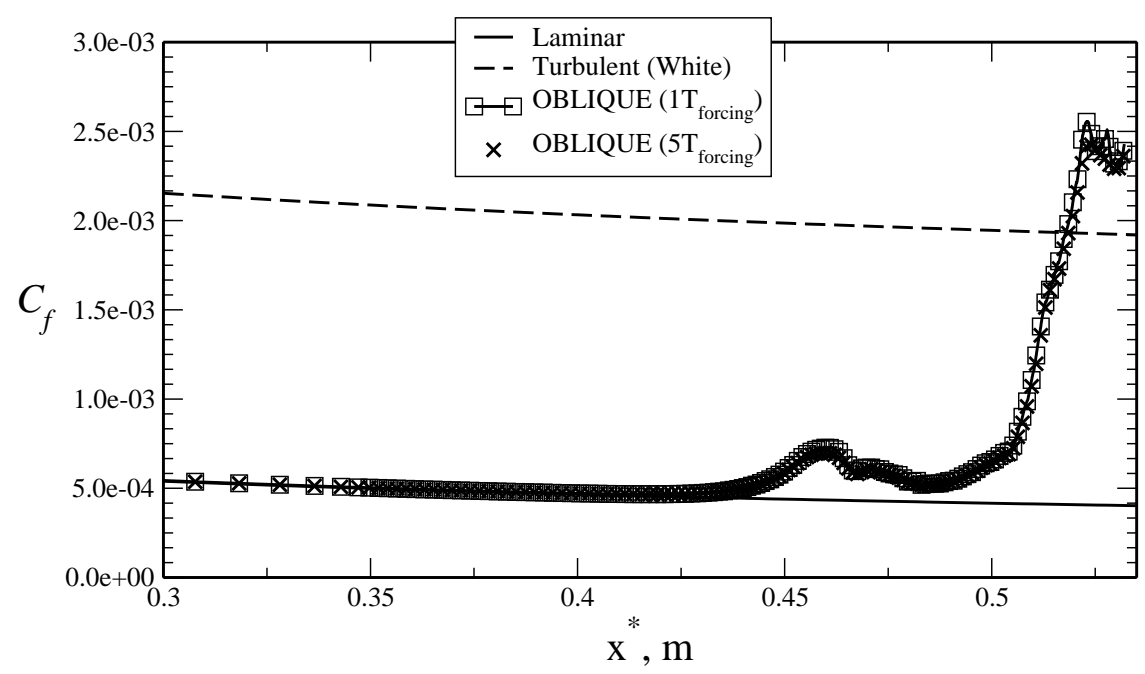

Figure 5.23: Time and azimuthal averaged skin friction coefficient from the oblique breakdown simulation. Note that the interval for time average is indicated by the number of forcing periods.

look strikingly different from those for fundamental breakdown. These streaks are a consequence of the steady modes (longitudinal vortex). In the case of oblique breakdown the nonlinearly generated steady modes are $(0,2),(0,4),(0,6)$, etc. Therefore the azimuthal spacing of these streaks are different for oblique breakdown. The first upstream appearance of the streaks is due to the steady mode $(0,2)$, this is the mode that reaches the highest amplitude first. There are about 36 streaks around the circumference of the cone and that spacing is directly related to the $(0,2)$ mode. Downstream close to the outflow, however, several higher steady modes $((0,4),(0,6)$, etc) have reached amplitudes higher than that of the $(0,2)$ mode. Hence, several streaks appear with a wide range of azimuthal spacing before starting to breakdown close to the end of the computational domain.

Flow structures from the oblique breakdown simulation are presented in figures 5.25 and 5.26. Figure 5.25 shows isosurface of Q criterion on the cone. Upstream close to the nose of the cone, flow structures at the forcing location are visible. Downstream of the forcing location, the linear regime is mainly influenced by the forced modes $(1,1)$. Thus the flow structures in the linear regime represent the superposition 

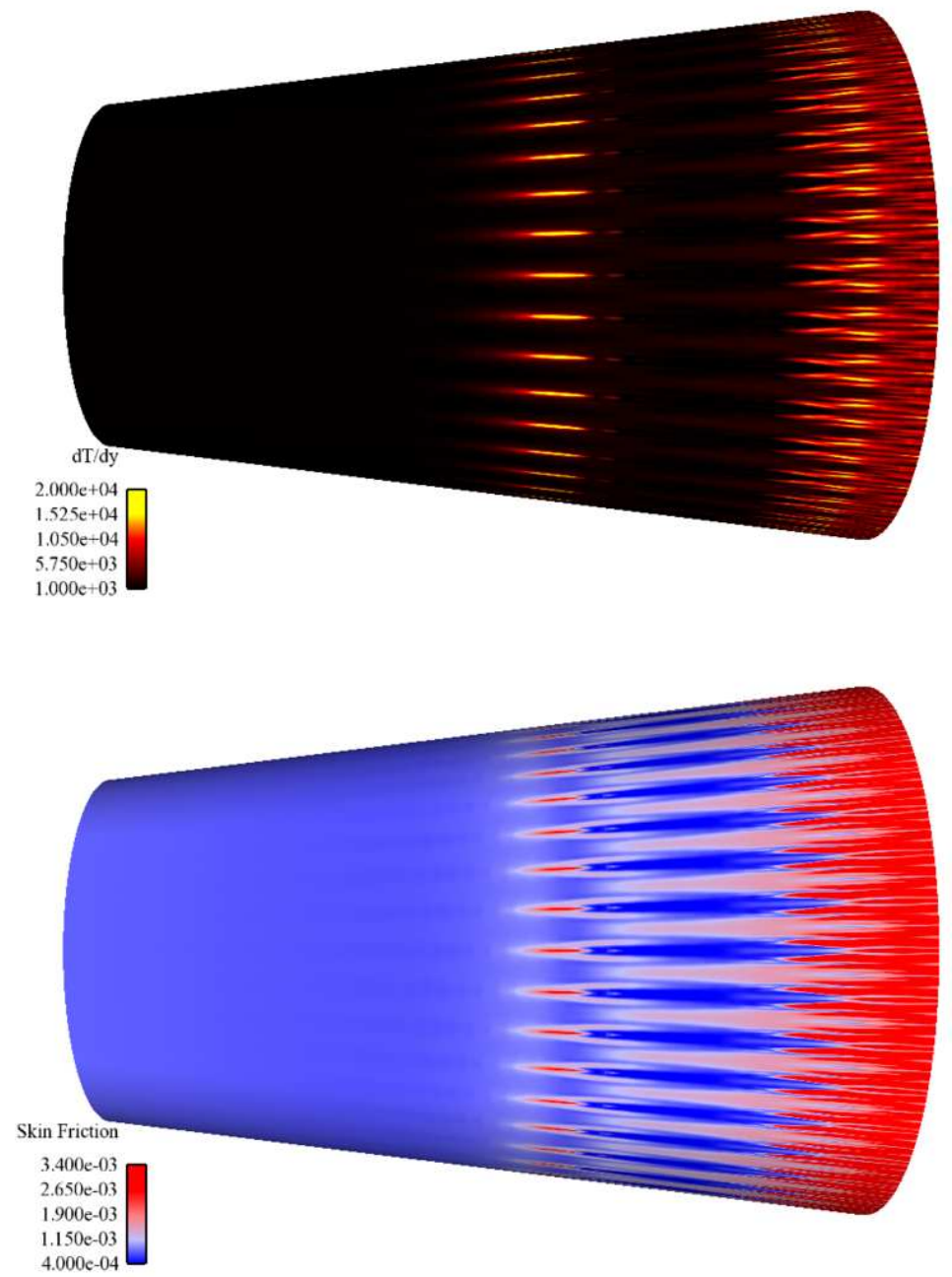

Figure 5.24: Time averaged heat transfer $(d T / d y)$ and skin friction at the wall from the oblique breakdown simulation. The streamwise aligned streaks observed in the case of oblique breakdown looks strikingly different from that observed for fundamental breakdown. 


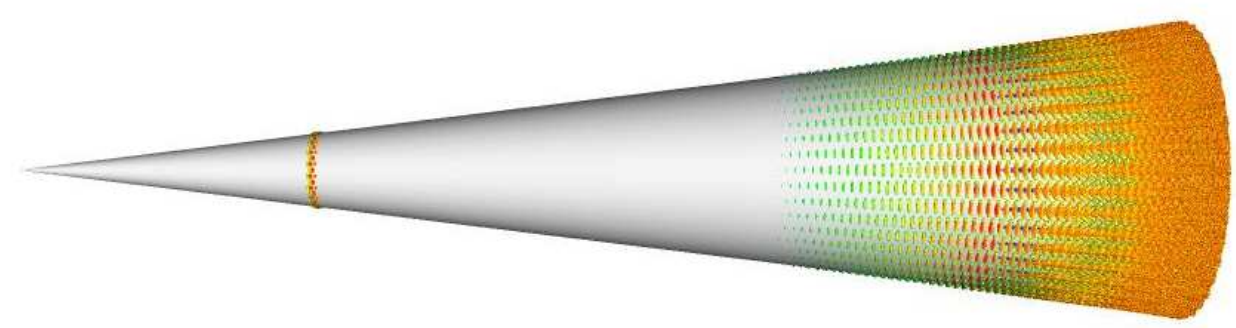

Figure 5.25: Visualization of flow structures by isosurface of $\mathrm{Q}$ criterion from the oblique breakdown simulation. The isosurface is colored using the streamwise velocity magnitude.

of both waves up to about $x^{*} \sim 0.44 m$. A checkerboard pattern, that is typical for a pair of oblique waves could be clearly seen on the isosurface shown in figures 5.26a. Then in the nonlinear regime generation of higher modes, especially the vortex modes $(0,2),(0,4),(0,6)$ alter the flow field. Finally as the nonlinear interactions become stronger, the flow breaks up into wedge-shaped regions where small-scale flow structures develop (see figures 5.26b).

In comparison to fundamental breakdown, the onset of transition, the initial rise and dip in skin friction and the final rise in skin friction in oblique breakdown show a similar trend. However, it is extremely difficult to compare transition onset between these two mechanisms because they are of a completely different nature. The true onset of transition for fundamental breakdown could be considered to occur after the first rise in skin friction because this initial distortion of the mean flow is caused entirely by the axisymmetric primary disturbance wave. With this consideration, the true onset of transition occurs later for fundamental breakdown. At this point, however, the slope of the skin friction is about the same for both breakdown scenarios. Thus, it is difficult to draw any concrete conclusions regarding the relative dominance of one transition mechanism over the other and it suffices to say that both are likely 


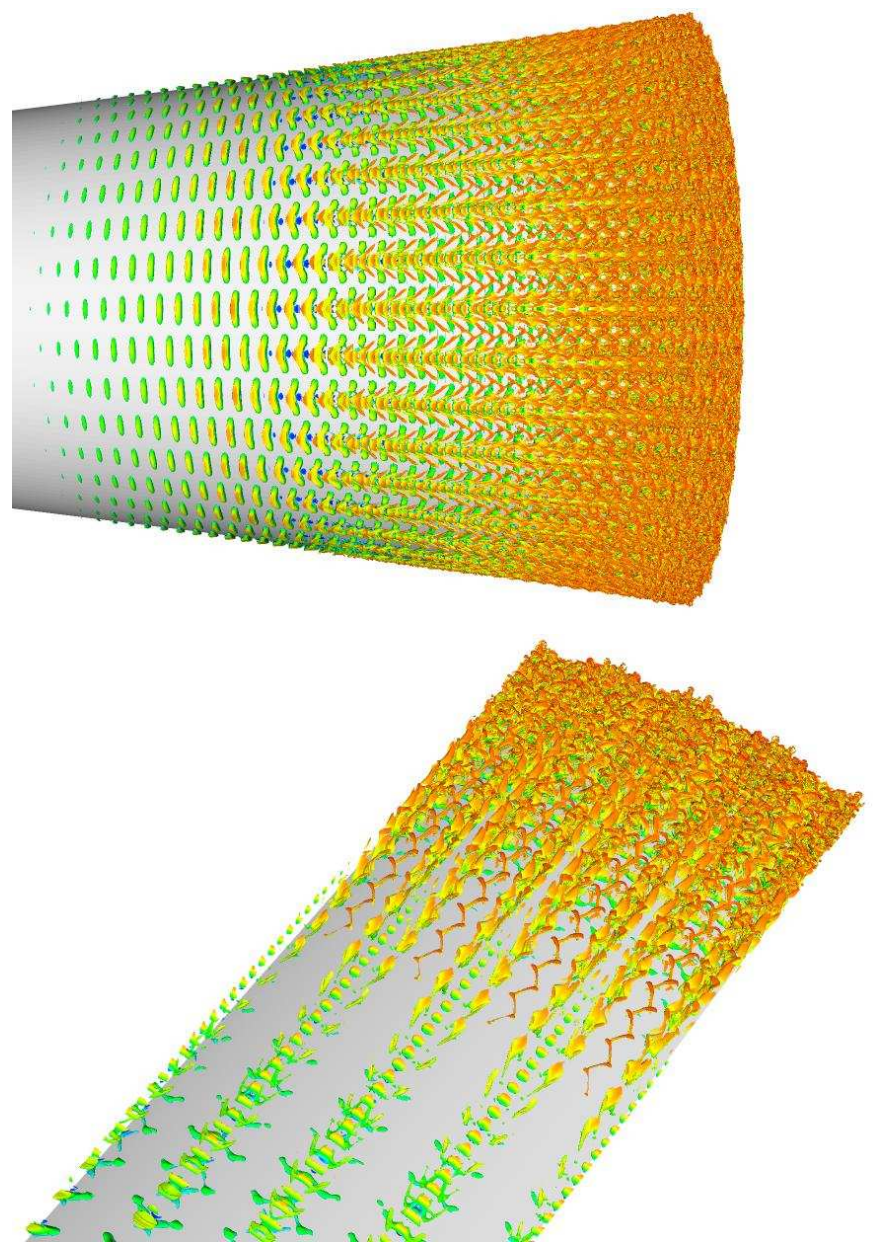

Figure 5.26: Close-up view of isosurface of $\mathrm{Q}$ criterion from the oblique breakdown simulation on the last downstream part of the cone (top) and at the late stage of transition (bottom). The isosurface is colored using the streamwise velocity magnitude. 


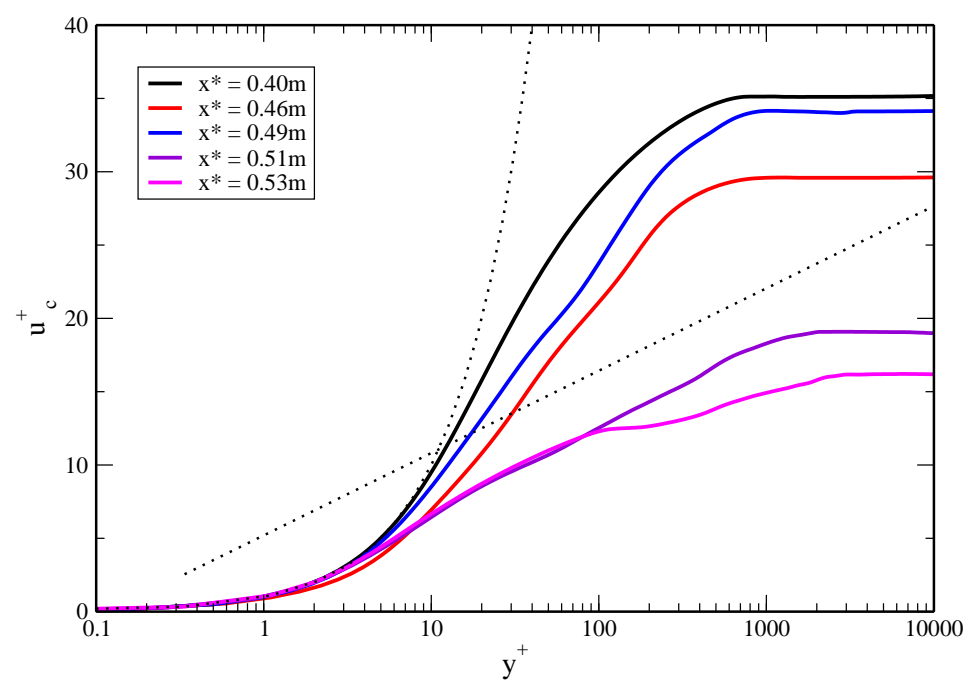

Figure 5.27: Van Driest transformed streamwise velocity profiles normalized with the friction velocity for different streamwise positions from the oblique breakdown simulation.

to be relevant for hypersonic boundary layers.

\subsection{Summary of controlled transition simulations}

The wave packet simulations provided strong evidence for a possible presence of fundamental and subharmonic resonance mechanisms in the nonlinear transition regime. However, the fundamental resonance was much stronger than the subharmonic resonance. In addition, strong peaks were observed for low-wave-number (shallow wave angle) second-mode oblique waves, which indicates a possible presence of oblique breakdown mechanism. Therefore controlled transition simulations were performed for fundamental resonance and oblique breakdown mechanisms. Fundamental resonance is mainly influenced by the forcing amplitude of the primary wave and the wave angle of the secondary oblique wave pair. For the cone geometry the wave angle of oblique disturbance waves changes in the downstream direction. Therefore, first a parameter study was performed to determine the most strongly resonating oblique wave pair. Several low-resolution simulations were performed for azimuthal wave 

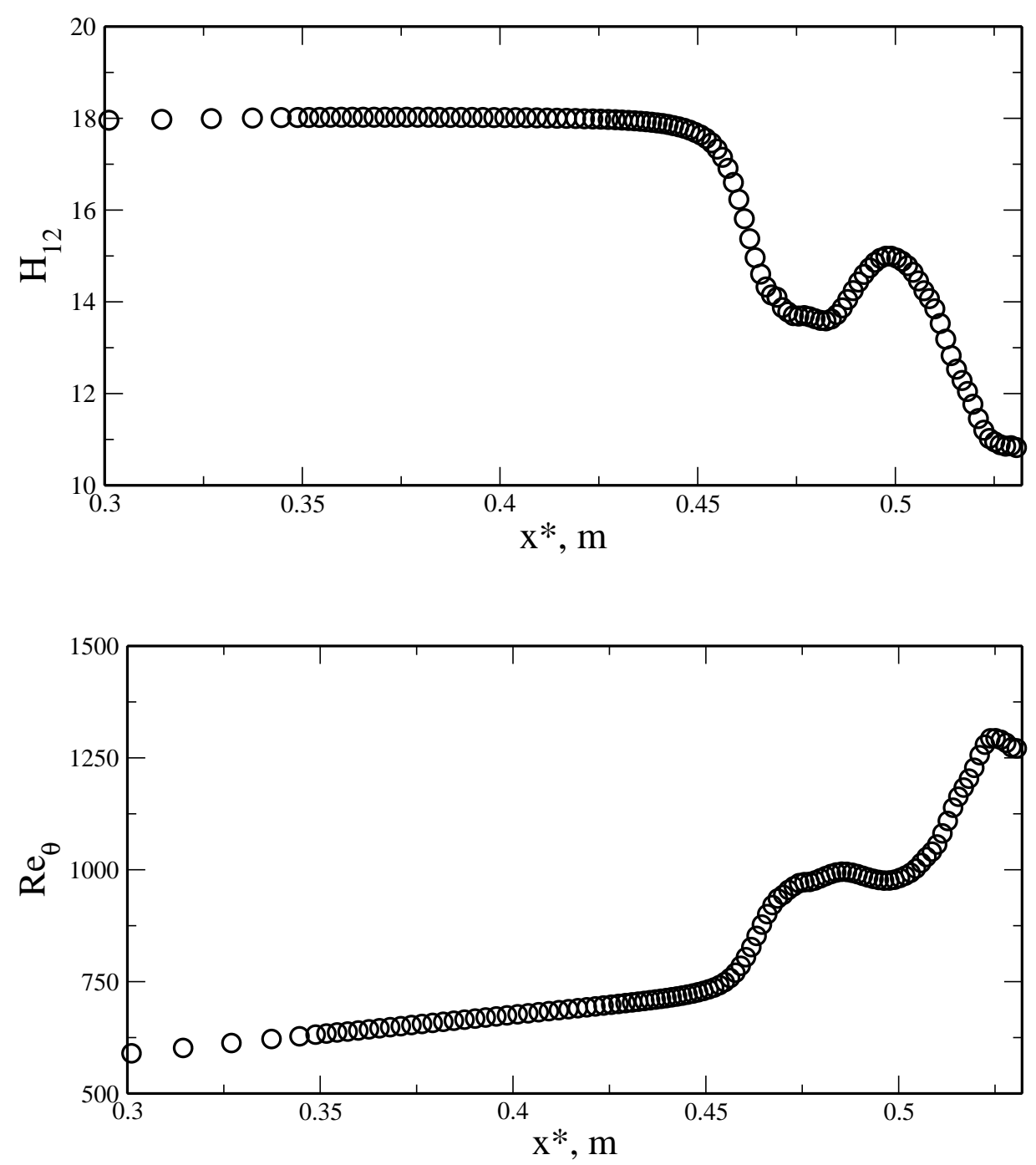

Figure 5.28: Downstream development of select mean-flow properties: (a) shape factor, $H_{12}$ (top) and (b) Reynolds number based on momentum thickness, $R e_{\theta}$ (bottom) from the oblique breakdown simulation. Time average was obtained for five forcing periods $\left(T_{\text {forcing }}\right)$. 

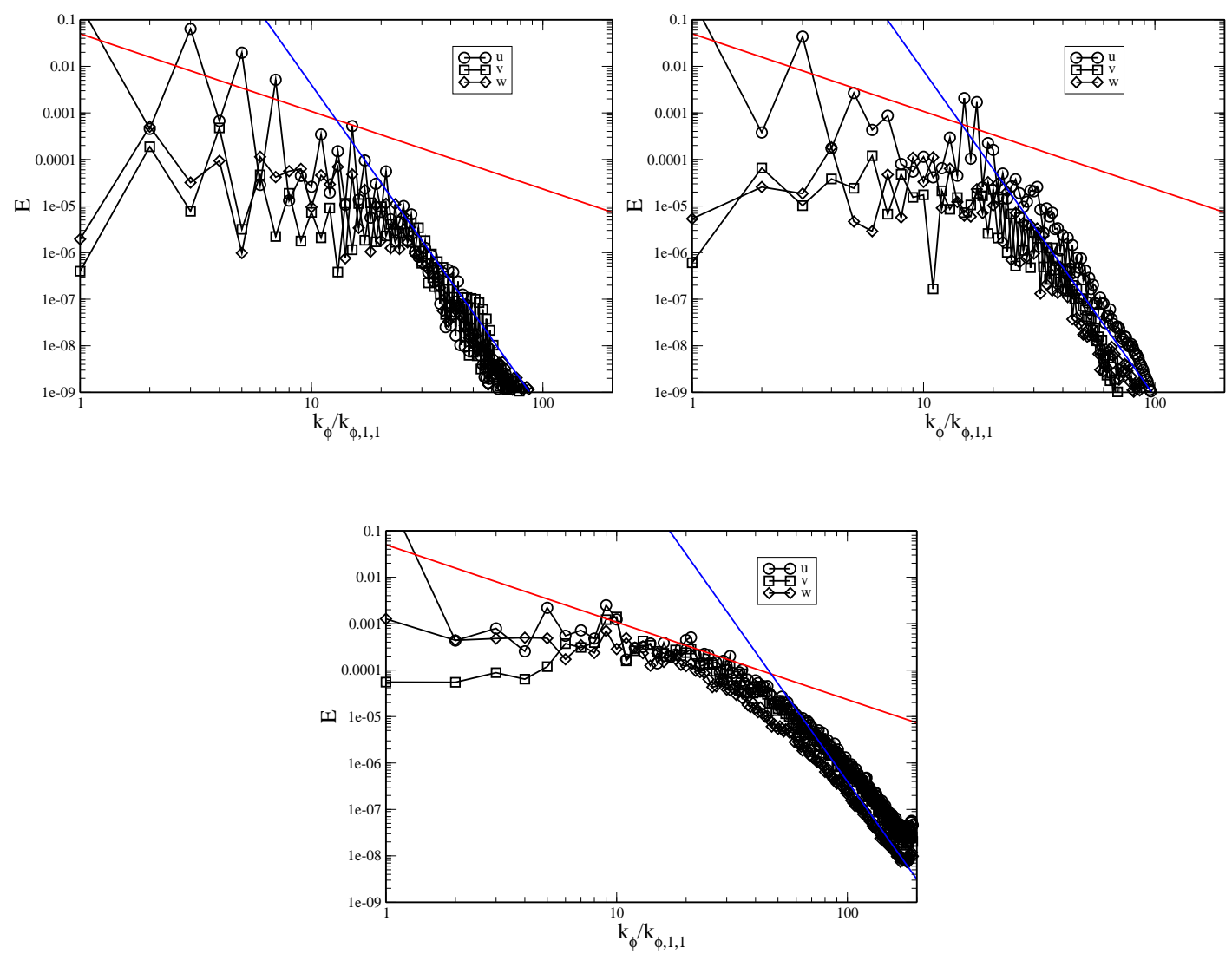

Figure 5.29: Energy spectra versus $k_{\phi}$ for $u, v$ and $w$ at (a) $x^{*}=0.47 m$, (b) $x^{*}=$ $0.49 \mathrm{~m}$ and $(\mathrm{c}) x^{*}=0.53 \mathrm{~m}$. The red and blue lines indicate the theoretical estimate for the decay of the energy spectrum: $E \sim k_{\phi}^{-5 / 3}$ (red line) and $E \sim k_{\phi}^{-7}$ (blue line). 
numbers $\left(k_{c}\right)$ ranging from 10 to 230 . In these simulations, the axisymmetric primary wave $(1,0)$ with frequency $F=1.071 \times 10^{-5}\left(f^{*}=210 \mathrm{kHz}\right)$ was forced at a moderate amplitude $\left(A_{1,0}=0.1 \%\right.$ of the free-stream velocity) and the secondary oblique waves $(1, \pm 1)$ were forced at a low-amplitude $\left(A_{1, \pm 1}=0.001 \%\right.$ of the free-stream velocity) From this parameter study, azimuthal mode number $k_{c}=150$ was identified as the most strongly resonating secondary wave.

A set of highly resolved "controlled" fundamental breakdown simulations were performed using the most strongly resonating oblique wave pair as secondary waves. The skin friction coefficient obtained from these simulations initially follows the laminar curve up to $x^{*} \sim 0.41 \mathrm{~m}$. Then the skin friction increases from the laminar curve towards the theoretical turbulent one. However, farther downstream the skin friction drops back to near the laminar curve before rising again steeply and even overshooting the theoretical turbulent curve close to the end of the computational domain. A closer look at the streamwise development of disturbance waves, revealed that the initial rise in skin friction is caused by the large-amplitude primary wave $(1,0)$. The first peak in the skin friction roughly corresponds to the streamwise location where the primary wave $(1,0)$ attains nonlinear saturation. As the primary wave starts to decay following the nonlinear saturation, the skin friction decreases strongly. Then, finally, a steep rise in skin friction occurs when all higher modes experience strong nonlinear amplification, and results in an overshoot of the theoretical turbulent curve.

Highly resolved oblique breakdown simulations were also performed to find out if it can indeed be a viable path to complete breakdown to turbulence. Secondmode oblique waves with azimuthal wave number $k_{c} \pm 20$ and frequency $F=1.071 \times$ $10^{-5}\left(f^{*} \approx 210 k H z\right)$ were forced to initiate the oblique breakdown. The time- and azimuthally-averaged skin friction coefficient obtained from this simulation follows the laminar curve up to $x^{*} \sim 0.44 m$. Then, there is a small rise, and then, a dip in the skin friction coefficient, which is similar to the initial rise and fall observed in the fundamental breakdown simulations. As in the case of fundamental breakdown, 
the initial rise here is also related to the high-amplitude primary wave. However, this initial rise is much smaller for oblique breakdown, which could be due to the lower linear growth and hence, the lower amplitude of the primary wave. Farther downstream, the skin friction rises again steeply and overshoots the turbulent skin friction curve. Quite similar to the non-monotonic behavior observed for fundamental breakdown. 


\section{SUMMARY AND CONCLUSIONS}

Laminar-turbulent transition in hypersonic boundary layers has a dramatic effect on heat transfer and skin friction. This effect is critical for reentry vehicles and for modern airbreathing hypersonic cruise vehicles. Yet, the physics of the transition process is still not understood well enough to be used for modelling and predicting transition. Hypersonic transition experiments are notoriously difficult and expensive to perform. On the other hand, direct numerical simulations of transition in highspeed boundary layers require large CPU time and high resolution. Hence, most of the earlier research efforts for hypersonic transition have focused on the receptivity and the linear stages of boundary layer transition. However, in hypersonic flows, due to compressibility, the nonlinear transitional flow regime may extend over a significant downstream extent of a flight vehicle. As a result, transition prediction tools based on the linear $\mathrm{N}$-factor calculations are not sufficient to estimate the location where the flow becomes fully turbulent. Hence, in order to develop new transition prediction tools which incorporate nonlinear transition physics, it is very important to understand the nonlinear stages of transition to turbulence in hypersonic boundary layers.

Numerical investigations, such as the work presented in this dissertation, compliment the experimental transition research efforts. Direct numerical simulations (DNS) of hypersonic boundary layer transition can provide data at a resolution and quality that cannot be obtained from laboratory experiments. Despite the large compute time and high grid resolution requirements, with recent advances in computer power and efficient parallelizable numerical algorithms, DNS has become a powerful tool for hypersonic boundary layer transition research. Hence, DNS is the method of choice for this research work and it is employed to investigate laminar-turbulent transition in a sharp cone boundary layer at Mach 6 . 
The objective of this research is to explore the dominant nonlinear mechanisms in a "natural" broad band disturbance environment and use this knowledge to perform controlled transition simulations to study these mechanisms in more detail. Towards this end, a "natural" transition scenario was modeled and investigated using wave packet disturbances. In order to generate a wide range of disturbance waves, the boundary layer is forced by a short duration (localized) blowing and suction pulse. The pulsed forcing leads to three-dimensional wave packets which travel in downstream direction. The flow parameters used in the simulations were based on the experimental conditions of the Boeing/AFOSR Mach 6 Quiet Tunnel at Purdue University.

The linear development of the wave packet was studied first, by pulsing the boundary layer with a low amplitude ( $0.001 \%$ of the free-stream velocity). Contours of instantaneous wall-pressure illustrate that near the disturbance source the wave packet has an entirely three-dimensional structure. However, as it propagates downstream stronger axisymmetric or two-dimensional wave fronts develop. In addition to these axisymmetric disturbance waves oblique waves were also observed on the lateral sides of the wave packet. However, the amplitudes of these oblique waves were small compared to the dominant axisymmetric waves. The wall-pressure disturbance spectrum exhibited a maximum at the azimuthal mode number $k_{c}=0$. The spectrum broadened in downstream direction as the lower frequency first mode oblique waves are amplified. However, the peak amplitude remained at $k_{c}=0$ which shifted to lower frequencies in the downstream direction. This was expected as for high Mach numbers axisymmetric or two-dimensional waves experience the highest streamwise amplification rate.

Then to investigate the weakly nonlinear transition regime, the boundary layer was forced with a medium amplitude pulse ( $0.5 \%$ of the freestream velocity). Snapshots of instantaneous wall-pressure disturbance contours illustrate that the wave packet develops linearly at first before the high amplitude two-dimensional wave fronts in 
the wave packet becomes modulated in the azimuthal direction due to nonlinear interactions. The wall pressure disturbance spectrum confirmed that the wave packet develop linearly at first. However, in the weakly nonlinear regime strong secondary peaks were observed at the fundamental frequency $f^{*} \approx 200 \mathrm{KHz}$ for larger azimuthal wave. This development may be an indication of a fundamental resonance mechanism. Higher harmonics $f^{*} \approx 400 \mathrm{KHz}$ of the high amplitude frequency band $f^{*} \approx 200 \mathrm{KHz}$ are also visible. Note that strong second harmonic disturbances were also observed in the experiments by Stetson et al. (1983). In addition, secondary peaks are observed at approximately half the frequency of the high amplitude frequency band, which would be an indication of a subharmonic resonance. However, the spectrum indicated that the fundamental resonance was stronger than subharmonic resonance. Strong peaks were also observed for low-wave-number second mode oblique waves which indicates a possible presence of oblique breakdown mechanism.

Finally, to identify more clearly which of these mechanisms are dominant in a strongly nonlinear wave packet and in particular to explore which mechanisms ultimately lead to nonlinear turbulent breakdown, a third simulation with a higher forcing amplitude (5\% of freestream velocity) was performed. The temporal and spatial development of the centerline wall-pressure disturbance amplitude indicates that the nonlinear wave packet still grows linearly at first before reaching the weakly nonlinear regime. Eventually, further downstream strong nonlinear interactions lead to breakdown into local patches of turbulent flow (turbulent spots) surrounded by otherwise laminar flow. The wall-pressure disturbance spectrum showed similar trends as seen for the weakly nonlinear wave packet and again, indicated that fundamental resonance and oblique breakdown might be the dominant nonlinear mechanism for the investigated flow.

A set of highly resolved "controlled" fundamental breakdown and oblique breakdown simulations were performed. The skin friction coefficient ob tained from the fundamental breakdown simulations initially follows the laminar curve up to $x^{*} \sim 0.41 \mathrm{~m}$. 
Then the skin friction increases from the laminar curve towards the theoretical turbulent one. However, farther downstream the skin friction drops back to nearly the laminar curve before rising again steeply and even overshooting the theoretical turbulent curve close to the end of the computational domain. Such nonmonotonic behaviour of skin friction has not been observed for low speed flows. A closer look at the results, revealed that the initial rise in skin friction is caused by the large amplitude primary wave $(1,0)$. The first peak in the skin friction roughly corresponds to the streamwise location where the primary wave $(1,0)$ saturates. As the primary wave starts to decay following the nonlinear saturation, the skin friction decreases strongly. Then finally, a steep rise in skin friction occurs when all higher modes experience strong nonlinear amplification, and results in an overshoot of the theoretical turbulent curve.

Oblique breakdown simulations produced skin friction curves that also showed similar trends. It initially follows the laminar curve up to $x^{*} \sim 0.44 m$. Then there is a small initial rise and then a dip in the skin friction coefficient, similar to the initial rise and fall observed in the fundamental breakdown simulations. As in the case of fundamental breakdown the initial rise in skin friction is related to the high amplitude primary wave. However, the rise is much smaller for oblique breakdown which could be due to the lower amplitude of the primary wave. Farther downstream the skin friction rises again steeply and overshoot the turbulent skin friction curve close to the end of the computational domain. Both fundamental breakdown and oblique breakdown produced streamwise streaks that looked qualitatively similar to the streaks observed in the experiments at Purdue University. Both mechanisms may have played a role in the natural "unforced" transition experiments conducted at Purdue and both fundamental breakdown and oblique breakdown may indeed be viable paths to complete breakdown to turbulence in hypersonic boundary layers at Mach 6. 


\section{REFERENCES}

Adams, N. A. \& Kleiser, L. 1993 Numerical simulation of transition in a compressible flat plate boundary layer. In Transitional and Turbulent Compressible Flows (ed. L. D. Kral \& T. A. Zang), FED 151, pp. 101-110. ASME.

Alavyoon, F., Henningson, D. S. \& Alfredsson, P. H. 1986 Turbulent spots in plane poiseuille flow-flow visualization. Phys. Fluids 29, 1328-1331.

Alba, C. R., Casper, K. M., Beresh, S. J. \& Schneider, S. P. 2010 Comparison of experimentally measured and computed second-mode disturbances in hypersonic boundary-layers. AIAA-2010-0897.

Anderson, J. D. 1997 A history of Aerodynamics and its impact on flying machines. Cambridge University Press.

Anderson, J. D. 2006 Hypersonic and high temperature gas dynamics, 2nd edn. American Institute of Aeronautics and Astronautics.

Balakumar, P. \& Malik, M. R. 1992 Discrete modes and continuous spectra in supersonic boundary layers. J. Fluid Mech. 239, 631-656.

Beckwith, I. E., Creel, T. R., Chen, F.-J. \& Kendall, J. M. 1983 Free stream noise and transition measurements in a Mach 3.5 pilot quiet tunnel. AIAA1983-0042.

Berridge, D., Chou, A., Ward, C., Steen, L., Gilbert, P., Juliano, T., Schneider, S. \& Gronvall, J. 2010 Hypersonic boundary-layer transition experiments in a Mach 6 quiet tunnel. AIAA-2010-1061.

Berry, S. A. \& Horvarth, T. J. 2007 Discrete roughness transition for hypersonic flight vehicles. AIAA-2007-0307.

Bountin, D., Shiplyuk, A. \& Maslov, A. 2008 Evolution of nonlinear processes in a hypersonic boundary layer on a sharp cone. J. Fluid Mech. 611, 427-442.

Carlson, D. R., Widnall, S. E. \& Peeters, M. F. 1982 A flow-visualization study of transition in plane Poiseuille flow. J. Fluid Mech. 121, 487-505.

Casper, K. M., Beresh, S. J., Henfling, J. F., Spillers, R. W., Pruett, B. \& SCHNeIDER, S. P. 2009 Hypersonic wind-tunnel measurements of boundarylayer pressure fluctuations. AIAA-2009-4054.

Casper, K. M., Beresh, S. J. \& Schneider, S. P. 2011 Pressure fluctuations beneath turbulent spots and instability wave packets in a hypersonic boundary layer. AIAA-2011-0372. 
Chen, F.-J., Malik, M. R. \& Beckwith, I. E. 1988 Comparison of boundarylayer transition on a cone and flat plate at Mach 3.5. AIAA-1988-0411.

Chen, F. J., Malik, M. R. \& Beckwith, I. E. 1989 Boundary-layer transition on a cone and flat plate at Mach 3.5. AIAA J. 27, 687-693.

Cohen, J. 1994 The initial evolution of a wave-packet in a laminar boundary layer. Phys. Fluids 6, 1133-1143.

Cohen, J., Breuer, K. S. \& Haritonidis, J. H. 1991 On the evolution of a wave packet in a laminar boundary layer. J. Fluid Mech. 225, 595-621.

Corke, T. C., Cavalieri, D. A. \& Matlis, E. 2002 Boundary-layer instability on sharp cone at Mach 3.5 with controlled input. AIAA J. 40 (5), 1015-1018.

Demetriades, A. 1960 An experiment on the stability of hypersonic laminar boundary layers. J. Fluid Mech. 7, 385-396.

Demetriades, A. 1977 Boundary layer instability observations at Mach number 7. J. Appl. Mech. 99, 7-10.

VAN DRIEst, E. 1952 Turbulent boundary layer on a cone in a supersonic flow at zero angle of attack. J. Aero. Sci. 19, 55-57.

Eissler, W. \& Bestek, H. 1996 Spatial numerical simulations of linear and weakly nonlinear wave instabilities in supersonic boundary layers. Theoret. Comput. Fluid Dyn. 8, 219-235.

Emmons, H. W. 1951 The laminar-turbulent transition in a boundary layer: Part I. J. Aero. Sci. 18.

Ermolaev, Y. G., Kosinov, A. D. \& Semionov, N. V. 1996 Experimental investigation of laminar-turbulent transition process in supersonic boundary layer using controlled disturbances. In Nonlinear Instability and Transition in ThreeDimensional Boundary Layers (ed. P. W. Duck \& P. Hall), pp. 17-26. Kluwer Academic Publishers.

Fasel, H., Thumm, A. \& Bestek, H. 1993 Direct numerical simulation of transition in supersonic boundary layer: Oblique breakdown. In Transitional and Turbulent Compressible Flows (ed. L. D. Kral \& T. A. Zang), FED 151, pp. 77-92. ASME.

Fasel, H. F. \& Konzelmann, U. 1990 Non-parallel stability of a flat-plate boundary layer using the complete Navier-Stokes equations. J. Fluid Mech. 221, 311-347. 
Fedorov, A. V. 2003 Receptivity of a high-speed boundary layer to acoustic disturbances. J. Fluid Mech. 491, 101-129.

Fedorov, A. V. 2011 Transition and stability of high-speed boundary layers. Ann. Rev. Fluid Mech. 43, 79-95.

Ferziger, J. H. 1998 Numerical Methods for Engineering Application, 2nd edn. Wiley-Interscience.

Fezer, A. \& Kloker, M. 1999 Spatial direct numerical simulation of transition phenomena in supersonic flat-plate boundary layers. In Laminar-Turbulent Transition (ed. H. F. Fasel \& W. S. Saric), pp. 415-420. Springer.

Fezer, A. \& Kloker, M. 2004 Grenzschichtumschlag bei Überschallströmung. Sonderforschungsbericht 259. DFG.

Fischer, M. C. 1972 Spreading of a turbulent disturbance. AIAA J. 10 (7), 957-959.

Fisher, D. F. \& Dougherty, N. S. 1982 In-flight transition measurement on a $10^{\circ}$ cone at Mach numbers from 0.5 to 2.0. TP 1971. NASA.

Forgoston, E. \& Tumin, A. 2005 Initial-value problem for three-dimensional disturbances in a compressible boundary layer. Phys. Fluids 17 (8).

Forgoston, E. \& Tumin, A. 2006 Three-dimensional wave packets in a compressible boundary layer. Phys. Fluids 18 (10).

Gasperas, G. 1987 The stability of the compressible boundary layer on a sharp cone at zero angle of attack. AIAA-1987-0494.

Gaster, M. 1975 A theoretical model of a wave packet in the boundary layer on a flat plate. Proc. Roy. Soc. 347 (1649), 271-289.

Gaster, M. \& Grant, I. 1975 An experimental investigation of the formation and development of a wave packet in a laminar boundary layer. Proc. Roy. Soc. 347 (1649), 253-269.

Glezer, A., Katz, Y. \& Wygnanski, I. 1989 On the breakdown of the wave packet trailing a turbulent spot in a laminar boundary layer. J. Fluid Mech. 198, $1-26$.

Goldstein, M. E. \& Hultgren, L. S. 1989 Boundary-Layer receptivity to longwave free-stream disturbances. Ann. Rev. Fluid Mech. 21, 137-166.

Gross, A. \& FAsel, H. F. 2008 High-order accurate numerical method for complex flows. AIAA J. 46, 204-214. 
Gad-el Hak, M., Blackwelder, R. F. \& Riley, J. J. 1981 On the growth of turbulent regions in laminar boundary layers. J. Fluid Mech. 110, 73-95.

HARRIS, P. J. 1997 Numerical investigation of transitional compressible plane wakes. $\mathrm{PhD}$ thesis, The University of Arizona.

Henningson, D. \& Kim, J. 1991 On turbulent spots in plane poiseuille flow. $J$. Fluid Mech. 228, 183-205.

Henningson, D., Spalart, P. \& Kim, J. 1987 Numerical simulations of turbulent spots in plane poiseuille and boundary-layer flow. Phys. Fluids 30, 2914-2917.

Husmeier, F. \& FAsel, H. F. 2007 Numerical investigations of hypersonic boundary layer transition for circular cones. AIAA-2007-3843.

Husmeier, F., Mayer, C. S. J. \& Fasel, H. F. 2005 Investigation of transition of supersonic boundary layers at Mach 3 using DNS. AIAA-2005-0095.

Jacobs, R. G. \& Durbin, P. A. 2001 Simulations of bypass transition. J. Fluid Mech. 428, 185-212.

Jocksch, A. \& KLeISER, L. 2008 Growth of turbulent spots in high-speed boundary layers on a flat plate. Int. J. Heat Fluid 29, 1543-1557.

Juliano, T. J., Schneider, S. P., Aradag, S. \& Knight, D. 2008 Quiet-flow ludwieg tube for hypersonic transition research. AIAA J. 46 (7), 1757-1763.

Katz, Y., Seifert, A. \& Wygnanski, I. 1982 On the evolution of the turbulent spot in a laminar boundary layer with a favourable pressure gradient. J. Fluid Mech. 221, 1-22.

KendalL, J. M. 1975 Wind tunnel experiments relating to supersonic and hypersonic boundary-layer transition. AIAA J. 13, 290-299.

Konzelmann, U. 1990 Numerische Untersuchungen zur räumlichen Entwicklung dreidimensionaler Wellenpakete in einer Plattengrenzschichtströmung. PhD thesis, Universität Stuttgart.

Konzelmann, U. \& FAsel, H. 1991 Numerical simulation of a three-dimensional wave packet in a growing flat plate boundary layer. In Boundary Layer Transition and Control, pp. 24.1-24.11.

Kosinov, A. D., Maslov, A. A. \& Semionov, N. V. 1997 An experimental study of generation of unstable disturbances on the leading edge of a plate at $\mathrm{M}=2 . J$. Appl. Mech. Tech. Phys. 38 (1), 45-50. 
Kosinov, A. D., Semionov, N. V. \& Shevelkov, S. G. $1994 a$ Investigation of supersonic boundary layer stability and transition using controlled disturbances. In Methods of Aerophysical Research (ed. A. M. Kharitonov), , vol. 2, pp. 159-166.

Kosinov, A. D., Semionov, N. V., Shevelkov, S. G. \& Zinin, O. I. $1994 b$ Experiments on the nonlinear instability of supersonic boundary layers. In Nonlinear Instability of Nonparallel Flows (ed. D. T. Valentine, S. P. Lin \& W. R. C. Philips), pp. 196-205. Springer.

Krishnan, L. \& Sandham, N. D. $2006 a$ Effect of Mach number on the structure of turbulent spots. J. Fluid Mech. 566, 225-234.

Krishnan, L. \& Sandham, N. D. $2006 b$ On the merging of turbulent spots in a supersonic boundary-layer flow. Int. J. Heat Fluid 27, 542-550.

Krishnan, L. \& Sandham, N. D. 2006c Turbulent spots in a compressible boundary-layer flow. In Proceedings of 6th IUTAM Symposium on LaminarTurbulent Transition (ed. R. Govindarajan). Springer.

Krishnan, L. \& Sandham, N. D. 2007 Strong interaction of a turbulent spot with a shock-induced separation bubble. Phys. Fluids 19.

Laible, A. C., Mayer, C. S. J. \& Fasel, H. F. 2008 Numerical investigation of supersonic transition for a circular cone at Mach 3.5. AIAA-2008-4397.

Laible, A. C., Mayer, C. S. J. \& Fasel, H. F. 2009 Numerical investigation of transition for a cone at mach 3.5: Oblique breakdown. AIAA-2009-3557.

VAN LEER, B. 1982 Flux-vector splitting for the Euler equations. In International Conference on Numerical Methods in Fluid Dynamics, , vol. 170, pp. 507-512. Springer-Verlag.

Lin, T. C., Grabowsky, W. R. \& Yelmgren, K. E. 1984 The search for optimum configurations for re-entry vehicles. Journal of Spacecraft and Rockets 21 (2), $142-149$.

MA, Y. \& Zhong, X. 2003 Receptivity of a supersonic boundary layer over a flat plate. part 1. wave structures and interactions. J. Fluid Mech. 488, 37-78.

MACK, L. M. 1965 Computation of the stability of the laminar compressible boundary layer. In Methods of Comp. Physics (ed. B. Alder, S. Fernbach \& M. Rotenberg), , vol. 4, pp. 247-299. Academic Press.

MACK, L. M. 1969 Boundary-layer stability theory. Internal Document 900-277. Jet Propulsion Laboratory, Pasadena, California. 
MACK, L. M. 1975 Linear stability theory and the problem of supersonic boundarylayer transition. AIAA J. 13, 278-289.

MACK, L. M. 1984 Boundary-layer linear stability theory. AGARD Report 709. Advisory Group for Aerospace Research and Development.

MACK, L. M. 1987 Stability of axisymmetric boundary layers on sharp cones at hypersonic mach numbers. AIAA-1987-1413.

MALIK, M. R. 1984 Instability and transition in supersonic boundary layers. In Laminar-Turbulent Boundary Layers (ed. E. M. Uram \& H. E. Weber), pp. 139 147.

Maslov, A. A., Shiplyuk, A. N., Sidorenko, A. A. \& Arnal, D. 2001 Leading-edge receptivity of a hypersonic boundary layer on a flat plate. J. Fluid Mech. 426, 73-94.

MAYer, C. S. J. 2009 Numerical investigation of the nonlinear transition regime in supersonic boundary layers. PhD thesis, The University of Arizona.

Mayer, C. S. J., von Terzi, D. A. \& Fasel, H. F. 2008 DNS of complete transition to turbulence via oblique breakdown at Mach 3. AIAA-2008-4398.

Mayer, C. S. J., von Terzi, D. A. \& Fasel, H. F. 2009 DNS of complete transition to turbulence via oblique breakdown at Mach 3: Part II. AIAA-20093558 .

Mayer, C. S. J., Wernz, S. \& FASel, H. F. 2007 Investigation of oblique breakdown in a supersonic boundary layer at Mach 2 using DNS. AIAA-2007-0949.

Medeiros, M. A. F. \& Gaster, M. $1999 a$ The influence of phase on the nonlinear evolution of wavepackets in boundary layers. J. Fluid Mech. 397, 259-283.

Medeiros, M. A. F. \& Gaster, M. $1999 b$ The production of subharmonic waves in the nonlinear evolution of wavepackets in boundary layers. J. Fluid Mech. 399, $301-318$.

Meitz, H. \& FASEL, H. F. 2000 A compact-difference scheme for the Navier-Stokes equations in vorticity-velocity formulation. J. Comp. Phys. 157, 371-403.

Morelli, E. A., Derry, S. D. \& Smith, M. S. 2005 Aerodynamic parameter estimation for the X-43A (Hyper-X) from flight data. AIAA-2005-5921.

Morkovin, M. V., Reshotko, E. \& Herbert, T. 1994 Transition in open flow systems - a reassessment. Bull. Am. Phys. Soc. 39 (1882). 
NARAsimha, R. 1985 The laminar-turbulent transition zone in the boundary layer. Prog. Aerospace Sci. 22, 81-111.

Peebles, C. 2008 Road to Mach 10: Lessons Learned from the X-43A Flight Research Program. American Institute of Aeronautics and Astronautics.

Peebles, C. 2011 Eleven Seconds into the Unknown: A History of the Hyper-X Program. American Institute of Aeronautics and Astronautics.

Poinsot, T. \& Lele, S. 1992 Boundary conditions for direct simulations of compressible viscous flows. J. Comp. Phys. 101, 104-129.

Pruett, C. D. \& Chang, C.-L. 1995 Spatial direct numerical simulation of highspeed boundary-layer flows. part II: Transition on a cone in Mach 8 flow. Theoret. Comput. Fluid Dyn. 7, 397-424.

Pruett, C. D. \& ZAng, T. A. 1992 Direct numerical simulation of laminar breakdown in high-speed, axisymmetric boundary layers. Theoret. Comput. Fluid Dyn. 3, 345-367.

Pruett, C. D., Zang, T. A., Chang, C.-L. \& Carpenter, M. H. 1995 Spatial direct numerical simulation of high-speed boundary-layer flows. part I: Algorithmic considerations and validation. Theoret. Comput. Fluid Dyn. 7, 49-76.

ReDA, D. C. 1979 Boundary-layer transition experiments on sharp, slender cones in supersonic free flight. AIAA J. 17 (8), 803-810.

Redford, J. A., Sandham, N. D. \& Roberts, G., T. 2011 Numerical simulations of turbulent spots in supersonic boundary layers: Effects of mach number and wall temperature. Prog. Aerospace Sci. In press.

Reshotko, E. 2008 Transition issues for atmospheric entry. Journal of Spacecraft and Rockets 45, 161-164.

Roy, C. J. \& Blottner, F. G. 2006 Review and assessment of turbulence models for hypersonic flows. Prog. Aerospace Sci. 42, 469-530.

Rufer, S. J. \& Schneider, S. P. 2006 Hot-wire measurements of instability waves on cones at Mach 6. AIAA-2006-3054.

SANDBERG, R. D. 2004 Numerical investigation of transitional and turbulent supersonic axisymmetric wakes. $\mathrm{PhD}$ thesis, The University of Arizona.

Sandham, N. D., Adams, N. A. \& Kleiser, L. 1995 Direct simulation of breakdown to turbulence following oblique instability waves in a supersonic boundary layer. Appl. Sci. Res. 54, 223-234. 
Saric, W. S., Reed, H. L. \& Kerschen, E. J. 2002 Boundary-Layer receptivity to freestream disturbances. Ann. Rev. Fluid Mech. 34, 291-319.

Schlatter, P. 2005 Large-Eddy simulation of transition and turbulence in wallbounded shear flow. PhD thesis, Swiss Federal Institute of Technology.

SCHNEIDER, S. P. 2001 Effects of high-speed tunnel noise on laminar-turbulent transition. Journal of Spacecraft and Rockets 38 (3), 323-333.

SchneIder, S. P. 2004 Hypersonic laminar-turbulent transition on circular cones and scramjet forebodies. Prog. Aerospace Sci. 40, 1-50.

SCHNeIder, S. P. 2006 Laminar-turbulent transition on reentry capsules and planetary probes. Journal of Spacecraft and Rockets 43 (6), 1153-1173.

SchneIDER, S. P. 2008 Development of hypersonic quiet tunnels. Journal of Spacecraft and Rockets 45 (4), 641-664.

SCHNEIDER, S. P. 2009 Private communication.

Schubauer, G. B. \& Klebanoff, P. S. 1956 Contributions on the mechanics of boundary layer transition. NACA Technical Report 1289.

Seifert, A. \& Wygnanski, I. 1995 On turbulent spots in a laminar boundary layer subjected to a self-similar adverse pressure gradient. J. Fluid Mech. 296, 185-209.

Shan, H., Ma, B., Zhang, Z. \& Nieuwstadt, F. 1999 Direct numerical simulation of a puff and a slug in transitional cylindrical pipe flow. J. Fluid Mech. 387, $39-60$.

Shiplyuk, A. N., Bountin, D. A., Maslov, A. A. \& Chokani, N. 2003 Nonlinear mechanisms of the initial stage of laminar-turbulent tansition at hypersonic velocities 44 (5), 1-12.

Smith, L. G. 1994 Pulsed-laser schlieren visualization of hypersonic boundary-layer instability waves. AIAA-1994-2639.

Stetson, K. F. \& Kimmel, R. L. 1992 On hypersonic boundary-layer stability. AIAA-1992-0737.

Stetson, K. F. \& Kimmel, R. L. 1993 On the breakdown of a hypersonic laminar boundary layer. AIAA-1993-0896.

Stetson, K. F., Thompson, E. R., Donaldson, J. C. \& Siler, L. G. 1983 Laminar boundary layer stability experiments on a cone at Mach 8. part I: Sharp cone. AIAA-1983-1761. 
Stetson, K. F., Thompson, E. R., Donaldson, J. C. \& Siler, L. G. 1984 Laminar boundary layer stability experiments on a cone at Mach 8. part II: Blunt cone. AIAA-1984-0006.

Stetson, K. F., Thompson, E. R., Donaldson, J. C. \& Siler, L. G. 1985 Laminar boundary layer stability experiments on a cone at Mach 8. part III: Sharp cone at angle of attack. AIAA-1985-0492.

Stetson, K. F., Thompson, E. R., Donaldson, J. C. \& Siler, L. G. 1986 Laminar boundary layer stability experiments on a cone at Mach 8. part IV: On unit reynolds number and environmental effects. AIAA-1986-1087.

Thumm, A. 1991 Numerische Untersuchungen zum laminar-turbulenten Strömungsumschlag in transsonischen Grenzschichtströmungen. $\mathrm{PhD}$ thesis, Universität Stuttgart.

Tumin, A. 2007 Three-dimensional spatial normal modes in compressible boundary layers. J. Fluid Mech. 586, 295-322.

White, F. M. 1991 Viscous Fluid Flow. McGraw-Hill.

Wygnanski, I., Haritonidis, J. H. \& Kaplan, R. E. 1979 On a tollmienschlichting wave packet produced by a turbulent spot. J. Fluid Mech. 92, 505-528.

Wygnanski, I., Sokolov, M. \& Friedman, D. 1976 On a turbulent 'spot' in a laminar boundary layer. J. Fluid Mech. 78, 785-819.

Wygnanski, I., Zilberman, M. \& Haritonidis, J. H. 1982 On the spreading of a turbulent spot in the absence of a pressure gradient. J. Fluid Mech. 123, 69-90.

Wygnanski, I. J. \& Champagne, F. H. 1973 On transition in a pipe. Part 1. The origin of puffs and slugs and the flow in a turbulent slug. J. Fluid Mech. 59, $281-335$.

ZhONG, X. 1998 High-order finite-difference schemes for numerical simulation of hypersonic boundary-layer transition. J. Comp. Phys. 144, 662-709.

Zhong, X. 2001 Leading-edge receptivity to free-stream disturbance waves for hypersonic flow over a parabola. J. Fluid Mech. 441, 315-367.

Zhong, X. \& MA, Y. 2006 Boundary-layer receptivity of Mach 7.99 flow over a blunt cone to free-stream acoustic waves. J. Fluid Mech. 556, 55-103.

Zhong, X. \& Tatineni, M. 2003 High-order non-uniform grid schemes for numerical simulation of hypersonic boundary-layer stability and transition. J. Comp. Phys. 190, 419-458. 
Zhong, X. \& WANG, X. 2012 Direct numerical simulation on the receptivity, instability, and transition of hypersonic boundary layers. Ann. Rev. Fluid Mech. 44, $527-561$. 